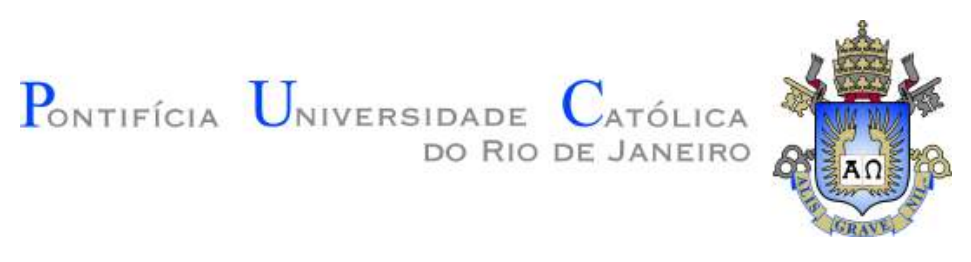

João Pedro Bezerra da Cunha

\title{
Persistence of straining in the four-roll mill flow
}

\author{
Dissertação de Mestrado
}

Dissertation presented to the Programa de Pós-graduação em Engenharia Mecânica, do Departamento de Engenharia Mecânica da PUC-Rio in partial fulfillment of the requirements for the degree of Mestre em Engenharia Mecânica.

Advisor : Prof. Paulo Roberto de Souza Mendes Co-advisor: Prof. Roney Thompson 


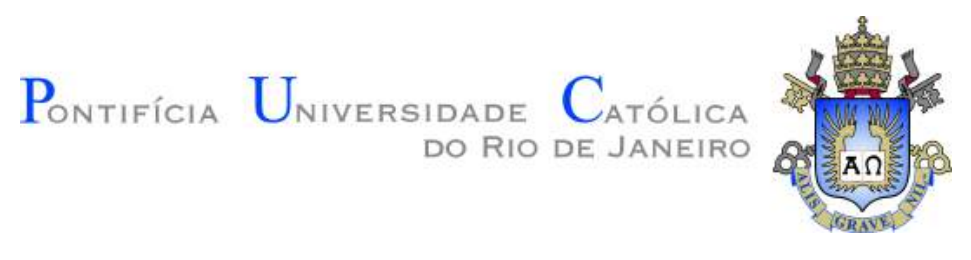

João Pedro Bezerra da Cunha

\section{Persistence of straining in the four-roll mill flow}

Dissertation presented to the Programa de Pós-graduação em Engenharia Mecânica da PUC-Rio in partial fulfillment of the requirements for the degree of Mestre em Engenharia Mecânica. Approved by the Examination Committee:

Prof. Paulo Roberto de Souza Mendes Advisor Departamento de Engenharia Mecânica - PUC-Rio

Prof. Roney Thompson Co-advisor Departamento de Engenharia Mecânica - COPPE/UFRJ

Prof. Márcio da Silveira Carvalho Departamento de Engenharia Mecânica - PUC-Rio

Dra. Priscilla Varges

Grupo de Reologia - PUC-Rio 
All rights reserved.

\section{João Pedro Bezerra da Cunha}

João Pedro Cunha graduated in Mechanical Engineering in the Pontifical Catholic University of Rio de Janeiro (PUCRio) in 2018. Then, he started to work as a research engineer with rheology and non-Newtonian fluid mechanics in the Rheology Group (GReo) at PUC-Rio, where he was already involved in research activities for two years as an undergraduate student. In parallel, he started his Master course in Mechanical Engineering also in PUC-Rio in 2019.

Bibliographic data

Cunha, J. P.

Persistence of straining in the four-roll mill flow / João Pedro Bezerra da Cunha; advisor: Paulo Roberto de Souza Mendes; co-advisor: Roney Thompson. - 2021.

128 f: il. color. ; $30 \mathrm{~cm}$

Dissertação (mestrado) - Pontifícia Universidade Católica do Rio de Janeiro, Departamento de Engenharia Mecânica, 2021.

Inclui bibliografia

1. Mechanical Engineering - Teses. 2. Emulsões. 3. Fourroll mill. 4. Classificação de escoamentos. 5. Deformação de gotas. I. de Souza Mendes, P. R.. II. Thompson, Roney L.. III. Pontifícia Universidade Católica do Rio de Janeiro. Departamento de Engenharia Mecânica. IV. Título. 


\section{Acknowledgments}

I would like to iniatially thank my advisor Paulo Roberto de Souza Mendes for our partnership along these last five years. You are an amazing example for me as a research engineer and also as person. I also wish to thank my co-advisor Roney Thompson for his huge contribution in this work.

Thanks to CAPES and PETROBRAS for having granted me a scholarship and the financial support that made it possible to conduct this research. This study was financed in part by the Coordenação de Aperfeiçoamento de Pessoal de Nível Superior - Brasil (CAPES) - Finance Code 001.

To my family, which keeps giving the necessary support for so long and are always present in the hardest moments, I love you.

I also need to recognize the incredible job from my fellow co-workers at the Rheology Group. Professor Mônica Nacacche, Priscilla Varges, Ricardo Leite, Tatiana Rochinha, Camila Moreira, João Pedro Naccache, Bruno Fonseca, Elias Rodrigues, Eliana Marin, Pedro Tobar, Roberta Kamei, Paulo de Henrique Lima, Julliana Karla, Sergio Ribeiro, Daniel Fonseca, Patrícia Almeida, João Paulo Nagoya, and Alexandre Fernandes especially.

To my longtime friends Lucas Sagrilo, Henrique Santiago, and Mateus Bastos who were present throughout so many journeys and certainly will be in the moments that the future holds, I will keep trusting you.

To the incredible friendships formed during my undergraduate program at PUC-Rio, which will be maintained for the rest of my life. Bernardo Barbosa, Marcelo Durães, Anna Leticia Alegria, Bruna Bergmann, Caio Fillipo, and Brunna Accardo, I am still hoping for the best for each one of you.

To my friends from our beloved Discord, your place is still special. João Pedro Sahione, Matheus Lourenço, Pedro de Sá, Lucas Fidalgo, Gabriel Bendia, João Victor Hollanda, Daniel Castello Branco, Lais Hasěk, João Pedro Coppelli, Rafael Hilst, and Gabriel Boquimpani manage to make the weeks incredibly easier.

To all of my friends who don't miss any Botafogo's game with me, my sincere thanks. There area lot of unforgettable stories driven by the same passion.

To all volunteers and students of the social project Elos Educação. It was such an honor to participate in this project which is extremly important in the lives of so many nice people.

And finally, exactly like my undegraduation final project, I dedicate this work to my beloved grandmother Yara Alexandrino, who is my greatest source of inspiration. 


\section{Abstract}

Cunha, J. P.; de Souza Mendes, P. R. (Advisor); Thompson, Roney L. (Co-Advisor). Persistence of straining in the four-roll mill flow. Rio de Janeiro, 2021. 128p. Dissertação de Mestrado - Departamento de Engenharia Mecânica, Pontifícia Universidade Católica do Rio de Janeiro.

The motivation of this work consists in the use of four-roll mill in order to increase the phase separation of water-in-oil emulsions $(\mathrm{W} / \mathrm{O})$ present in the primary process of oil industry. With mass and momentum conservation, the continuous phase is modeled by an incompressible, bi-dimensional and isothermal flow. Numerical simulations employing the finite element method were implemented to reveal the influence of the several flow configurations in the material mechanical behavior. From the obtained results, the standard way of classifying the flow in the four-roll mill according to the literature was proved inefficient. This work suggests local flow classifications for each position depending if it is occupied by the continuous or dispersed phase. The effect of the dispersed phase was described by a post-processing scheme. Microelements in shape of vectors were inserted in the domain and their deformations and pathlines were investigated. Thus, the deformation of droplets and their respective influences in the emulsion instability were analyzed.

\section{Keywords}

Emulsions; Four-roll mill; Flow classification; Droplet deformation. 


\section{Resumo}

Cunha, J. P.; de Souza Mendes, P. R.; Thompson, Roney L.. Persistência de deformação no escoamento no four-roll mill. Rio de Janeiro, 2021. 128p. Dissertação de Mestrado - Departamento de Engenharia Mecânica, Pontifícia Universidade Católica do Rio de Janeiro.

A motivação deste trabalho consiste no uso do four-roll mill para aumentar a separação de fases de emulsões água em óleo (A/O) presente no processamento primário da indústria de petróleo. A partir da conservação de massa e momento, a fase contínua foi modelada como escoamento incompressível, bi-dimensional e isotérmico. Simulações numéricas utilizando o método de elementos finitos foram implementadas para revelar a influência das diversas configurações de escoamento no comportamento mecânico do material. A partir dos resultados obtidos, a habitual forma de classificar o escoamento no four-roll mill de acordo com a literatura se demonstrou ineficiente. Este trabalho sugere classificações locais de escoamento a cada posição dependendo se a mesma está ocupada pela fase contínua ou dispersa da emulsão. O efeito da fase dispersa é descrito via pós-processamento. Microelementos no formato de vetores foram inseridos no domínio e investigou-se suas deformações e trajetórias. Consequentemente, analisou-se a deformação de gotas e a sua respectiva influência na instabilidade da emulsão.

\section{Palavras-chave}

Emulsões; Four-roll mill; Classificação de escoamentos; Deformação de gotas. 


\section{Table of contents}

$\begin{array}{llr}1 & \text { Introduction } & 11\end{array}$

1.1 Motivation 11

$\begin{array}{lll}1.2 & \text { Objectives } & 12\end{array}$

$\begin{array}{lll}1.3 & \text { Outlines } & 12\end{array}$

$2 \quad$ Background and literature $\quad 13$

$\begin{array}{lll}2.1 & \text { Emulsions } & 13\end{array}$

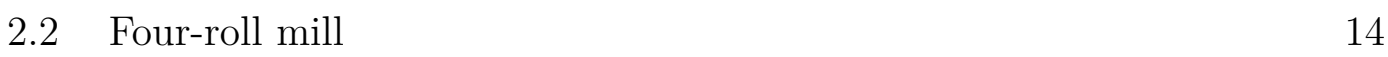

$\begin{array}{ll}2.3 \text { Literature review } & 16\end{array}$

$\begin{array}{lll}2.3 .1 & \text { Four-roll mill and drop deformation } & 17\end{array}$

$\begin{array}{lll}2.3 .2 & \text { Flow classification } & 18\end{array}$

3 Model description $\quad 22$

3.1 Domain and meshes $\quad 22$

$\begin{array}{ll}3.2 & \text { Modeling the flow }\end{array}$

$\begin{array}{ll}3.3 & \text { Weak formulation } \\ 3.4 & 24\end{array}$

3.4 Element 24

3.5 Boundary conditions 25

$\begin{array}{ll}3.6 & \text { Persistence of straining } \\ 3.7 & 28\end{array}$

$\begin{array}{lll}3.7 & \text { Microelement's deformation } & 30\end{array}$

$\begin{array}{lll}3.8 & \text { Non-dimensionalization } & 32\end{array}$

4 Results 33

4.1 Classic four-roll mill 33

4.1.1 Velocity fields 33

$\begin{array}{lll}\text { 4.1.2 } & \text { Streamlines } & 37\end{array}$

4.1.3 The normalized persistence-of-straining parameter field 40

4.1.4 The modified normalized persistence-of-straining parameter field 43

4.2 Expanded four-roll mill $\quad 48$

4.2.1 Velocity fields $\quad 48$

$\begin{array}{lll}4.2 .2 & \text { Streamlines } & 56\end{array}$

4.2.3 The normalized persistence-of-straining parameter fields 60

4.2.4 The modified persistence-of-straining parameter fields 66

4.2.5 Pathline and deformation of microelements 69

$5 \quad$ Final Remarks $\quad \mathbf{8 0}$

5.1 Future Works $\quad 81$

$\begin{array}{ll}\text { A Codes } & 87\end{array}$

$\begin{array}{lll}\text { A.1 Meshes } & 87\end{array}$

$\begin{array}{lll}\text { A.2 Simulations } & 95\end{array}$ 


\section{List of figures}

Figure 1.1 Simplified schematics of the primary process 12

Figure 2.1 Schematics of the emulsion water-in-oil (W/O) 13

Figure 2.2 Schematics related to destabilization of emulsions [1] $\quad 14$

$\begin{array}{lll}\text { Figure 2.3 Schematics of the four-roll mill [2] } & 15\end{array}$

Figure 2.4 Streamlines of homogeneous two-dimensional flow [3] 16

Figure 3.1 Classic four-roll mill mesh generated by gmsh. 22

Figure 3.2 Expanded four-roll mill mesh generated by gmsh. 23

$\begin{array}{lll}\text { Figure 3.3 } & \text { Mixed lagrange element } & 25\end{array}$

$\begin{array}{lll}\text { Figure 3.4 Borders of the classic four-roll mill. } & 26\end{array}$

Figure 3.5 Borders of the expanded four-roll mill. 27

Figure 4.1 Non-dimensional velocity field in the classic four-roll mill with distinct flow configurations.

Figure 4.2 Streamlines in the classic four-roll mill with distinct flow configurations.

Figure $4.3 \mathcal{R}^{*}$ field in the classic four-roll mill with distinct flow configurations.

Figure $4.4 \mathcal{R}^{*}{ }_{\text {mod }}$ field in the classic four mill with $\lambda=1$ and distinct microelements.

Figure 4.5 $\mathcal{R}^{*}{ }_{\text {mod }}$ field in the classic four mill with $\lambda=0$ and distinct microelements.

Figure $4.6 \mathcal{R}^{*}{ }_{\text {mod }}$ field in the classic four mill with $\lambda=-1$ and distinct microelements.

Figure 4.7 Velocity field in the expanded four-roll mill with $\lambda=1$ and distinct entrance Reynolds number.

Figure 4.8 Velocity field in the expanded four-roll mill with $\lambda=0$ and distinct entrance Reynolds number.

Figure 4.9 Velocity field in the expanded four-roll mill with $\lambda=-1$ and distinct entrance Reynolds number.

Figure 4.10 Streamlines in the expanded four-roll mill with $R e_{e n t}=0$ and distinct flow configurations.

Figure 4.11 Streamlines in the expanded four-roll mill with $R e_{\text {ent }}=5$ and distinct flow configurations.

Figure $4.12 \mathcal{R}^{*}$ field in the expanded four-roll mill with $R e_{\text {ent }}=0$ and distinct flow configurations.

Figure $4.13 \mathcal{R}^{*}$ field in the expanded four-roll mill with $R e_{\text {ent }}=5$ and distinct flow configurations.

Figure $4.14 \mathcal{R}^{*}{ }_{\text {mod }}$ field in the expanded four-roll mill with $R e_{\text {ent }}=0$, $\lambda=1$, and distinct microelements.

Figure $4.15 \mathcal{R}^{*}{ }_{\text {mod }}$ field in the expanded four-roll mill with $R e_{\text {ent }}=5$, $\lambda=1$, and distinct microelements. 
Figure 4.16 Pathlines in the expanded four-roll mill with $R e_{\text {ent }}=5$, $\lambda=1$, and distinct microelements placed at different initial vertical positions.

Figure $4.17 x^{*}$ versus $t^{*}$ (left column) and $y^{*}$ versus $t^{*}$ (right column) in expanded four-roll mill, for $R e_{e n t}=5, \lambda=1, y_{0}{ }^{*}=0$, and distinct microelements.

Figure $4.18 R^{*}$ versus $t^{*}$ in the expanded four-roll mill with $R e_{e n t}=$ $5, \lambda=1, y_{o}{ }^{*}=0$, and distinct microelements.

Figure 4.19 Pathlines in the expanded four-roll mill, for $R e_{e n t}=5$, $\lambda=0$ and distinct microelements.

Figure $4.20 x^{*}$ versus $t^{*}$ (left column) and $y^{*}$ versus $t^{*}$ (right column) in the expanded four-roll mill, for $R e_{e n t}=5, \lambda=0, y_{o}{ }^{*}=-1.57$, and distinct microelements.

Figure $4.21 R^{*}$ versus $t^{*}$ in the expanded four-roll mill, for $R e_{\text {ent }}=5$, $\lambda=0, y_{o}{ }^{*}=-1.57$, and distinct microelements. 


\section{List of tables}

Table 2.1 Flow classification with flow-type parameter [3] 15

Table 3.1 Dimensions used in both domains. 23

Table 3.2 Boundary conditions in the classic four-roll mill. 26

Table 3.3 Boundary conditions in the expanded four-roll mill. 27

Table 4.1 Dimensionless parameters used in the four-roll mill simulations. 


\section{Introduction}

\section{1}

\section{Motivation}

Emulsions are widely found in various stages of the oil industry, such as drilling, production, transportation, and processing of crude oils [4]. Furthermore, some techniques of enhanced oil recovery require external emulsions to displace the oil at reservoirs [5].

The production of an oil well varies substantially over time. Initially, the well produces an oil with a high level of purity. However, the concentration of water gradually increases, resulting in co-production [6]. Typically, the amount of water, in volume, that emulsifies is up to $20 \%$ for crude light oils and up to $35 \%$ for medium and heavy oils [7].

The production of oil in the form of an emulsion is a major problem for the industry due to the increases in costs related to transportation and processing. Water-in-oil emulsions have a greater viscosity compared to a dehydrated oil [8]. Consequently, it increases the consumption of energy since a higher pressure is necessary to transport the oil.

Moreover, after the transportation, emulsions must be submitted to a primary process to separate the water from oil and avoid problems related to the presence of water in the refineries [9]. Usually, in the primary process, the emulsion stays at the gravity settler. It relies on the difference in density between the fluids. The removed water from the emulsion is directed to a hydrocyclone before being discarded. The remaining fluid goes to an electrostatic coalescer as can be seen in Figure 1.1. Since the oil is not conductive and the water is, the droplets of water coalesce to form larger drops.

In order to increase the efficiency of the primary process and considering some financial drawbacks of the electrostatic coalescer, such as high energy consumption and high maintenance expenses, it is important to look for alternatives that produce similar effects on the emulsions. The main motivation of this work is to evaluate the capacity of the four-roll mill as an alternative mechanism to provide phase separation due to the fact that it provides several types of flow. 


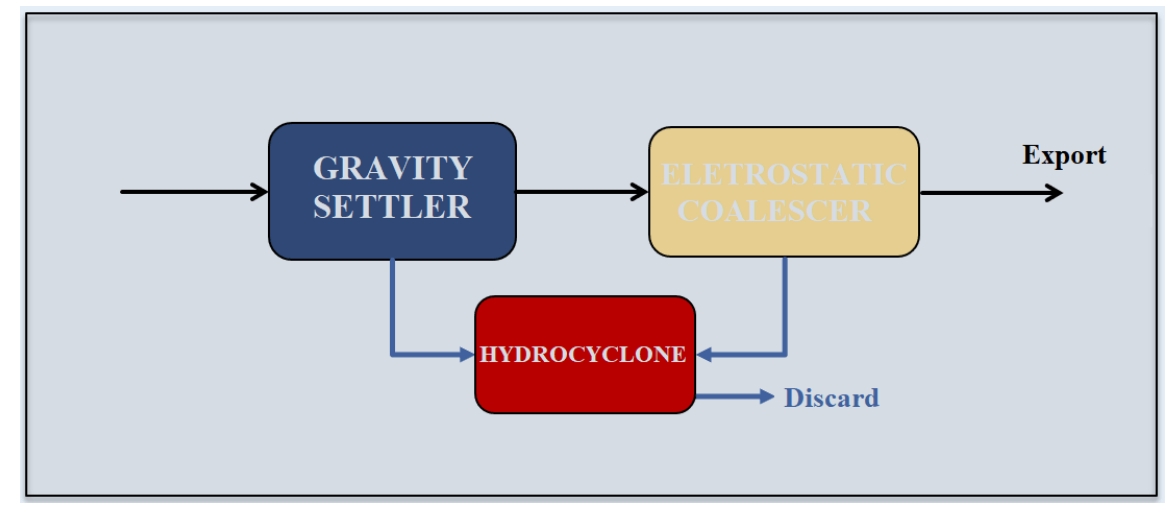

Figure 1.1: Simplified schematics of the primary process

\section{2}

\section{Objectives}

The main objective of this work is to develop a numerical simulation of the steady-state flow of a Newtonian fluid (continuous phase of emulsion) in the four-roll mill and analyze the deformation of small droplets (dispersed phase of emulsion) with distinct flow configurations. A local, frame-indifferent, and not restricted to particular classes of flows, classification criteria is used to question the classic point of view presented in the four-roll mill literature.

\section{3}

\section{Outlines}

This thesis is divided into four more chapters besides this first one. In Chapter 2, the main concepts and definitions about both emulsions and fourroll mill are presented. In the sequence, the four-roll mill, drop deformations, and flow classification are considered in the literature review. Chapter 3 presents the complete and detailed model description developed during the research. Following, Chapter 4 outlines the results of the simulations and the differences with previous works are discussed. Finally, Chapter 5 reveals the main conclusions of the present research and point out some interesting possibilities of future works related to this research. 


\section{2}

\section{Background and literature}

This chapter provides some basic concepts to introduce the theory related to emulsions and the four-roll mill apparatus, followed by a review of the literature in touch with these topics and flow classification criteria.

\section{1}

\section{Emulsions}

Emulsions are dispersions that characterize a mixture of two immiscible liquids. One of the liquids is presented as droplets and is dispersed in another liquid phase, which represent respectively the dispersed and continuous phases. This work motivation is based on an emulsion water-in-oil $(\mathrm{W} / \mathrm{O})$, as displayed in Figure 2.1.

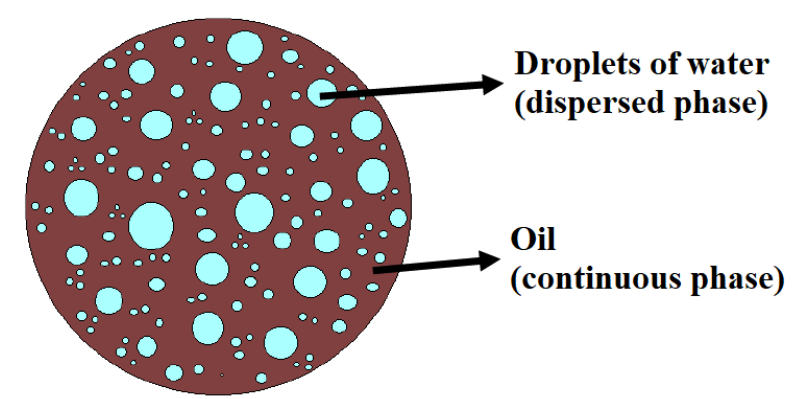

Figure 2.1: Schematics of the emulsion water-in-oil (W/O)

The system of two liquid phases is thermodynamically unstable since there is a natural energetic tendency of reducing its interfacial area, and consequently, its interfacial energy $[10,11]$. Therefore, in the absence of a stabilization mechanism, emulsions will tend to separate the phases.

Emulsions instability appears on several spontaneous processes which are represented in Figure 2.2. This is such a complex phenomenon to interpret because it requires an understanding of the variety of forces involved. Besides, many of these processes could happen simultaneously, complicating, even more, the analysis. Typically, the relevant parameters which rule these processes are the droplets size distribution, difference of density between the dispersed and continuous phases, difference of magnitude among attractive and repulsive 
forces, solubility of the dispersed phase in the continous, and stability of the liquid film between droplets.

Emulsifiers are external elements introduced in an emulsion system which modifies interfacial tension, in order to increase the stability of the liquid film between droplets. Emulsifiers belong to a wide group of compounds defined as surfactants that have a polar and a non-polar part. The molecular structure and concentration of these elements play a significantly role in the formation and stability of the emulsions [12].

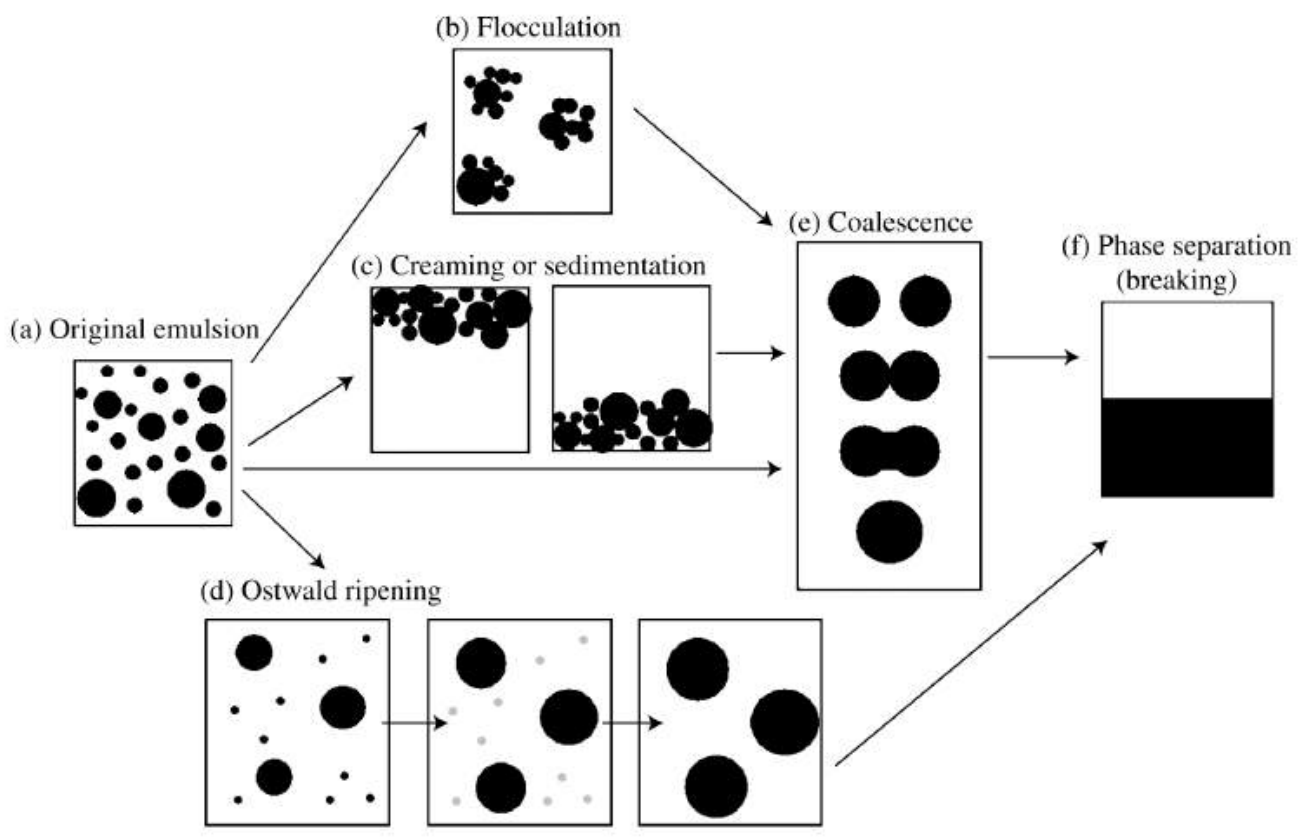

Figure 2.2: Schematics related to destabilization of emulsions [1]

\section{2}

\section{Four-roll mill}

The four-roll mill is typically defined in the literature as four cylinders of radius $a$, centered on the corners of a square of side $2 b$, immersed in square tank of side $2 l$ as depicted in Figure 2.3. 


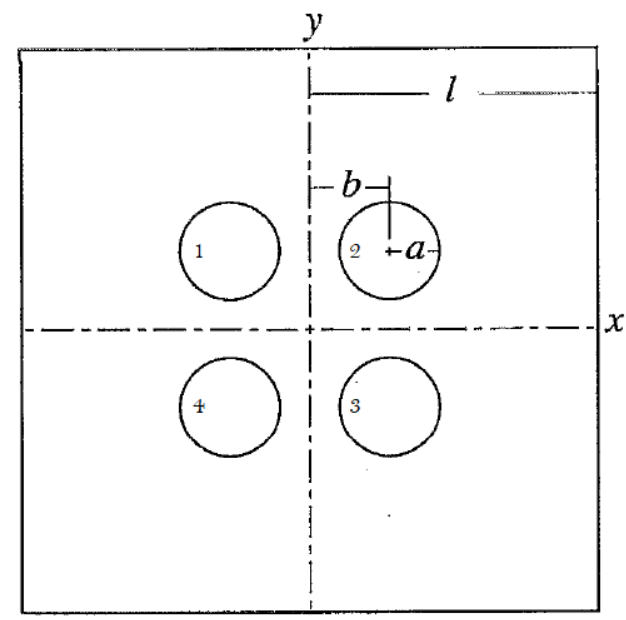

Figure 2.3: Schematics of the four-roll mill [2]

The main benefit of the four-roll mill, which justifies its recurrent use in experimental and numerical applications, consists of providing distinct possibilities of homogeneous two-dimensional flows in the region between rollers. A variety of flow configurations is generated by adjusting the angular speed and direction of the rollers.

The rollers are numbered in a clockwise direction, starting with the upper left one, as displayed in Figure 2.3. The flow-type parameter $\lambda$ is defined by the negative ratio of angular speeds belonging to non-adjacent pairs of rollers. Therefore, it is implied that crosswise pairs of them have the same angular velocity, ie, $\omega_{r 1}=\omega_{r 3}$ and $\omega_{r 2}=\omega_{r 4}$.

$$
\lambda=-\frac{\omega_{r 1, r 3}}{\omega_{r 2, r 4}}
$$

Despite the fact that the flow-type parameter varies between -1 and 1 , i.e., $\lambda \in[-1,1]$, Table 2.1 exhibits the flow classification that a few specific flow-type parameters lead. This criteria will be questioned in this work.

\begin{tabular}{cc}
\hline flow type & $\lambda$ \\
\hline pure extensional flow & 1 \\
\hline simple shear flow & 0 \\
\hline rigid body motion & -1 \\
\hline
\end{tabular}

Table 2.1: Flow classification with flow-type parameter [3]

In Figure 2.4, schematics of streamlines are presented according to each flow-type parameter [3]. Notice that for $\lambda=1$, there are hyperbolas with perpendicular asymptotes. In fact, for $0<\lambda \leq 1$, the angle between 
asymptotes is calculated by the relationship $\tan \left(\frac{\phi}{2}\right)=\sqrt{\lambda}$. On the other hand, for $-1 \leq \lambda<0$, the streamlines are closed ellipses and the ratio of the minor axis $b_{\min }$ and the major axis $a_{m a j}$ is given by the expression $\frac{b_{\min }}{a_{m a j}}=\sqrt{-\lambda}$. Thus, for $\lambda=-1$, the streamlines are circumferences. For every single flow type displayed, the center of the four-roll mill is a stagnation point.

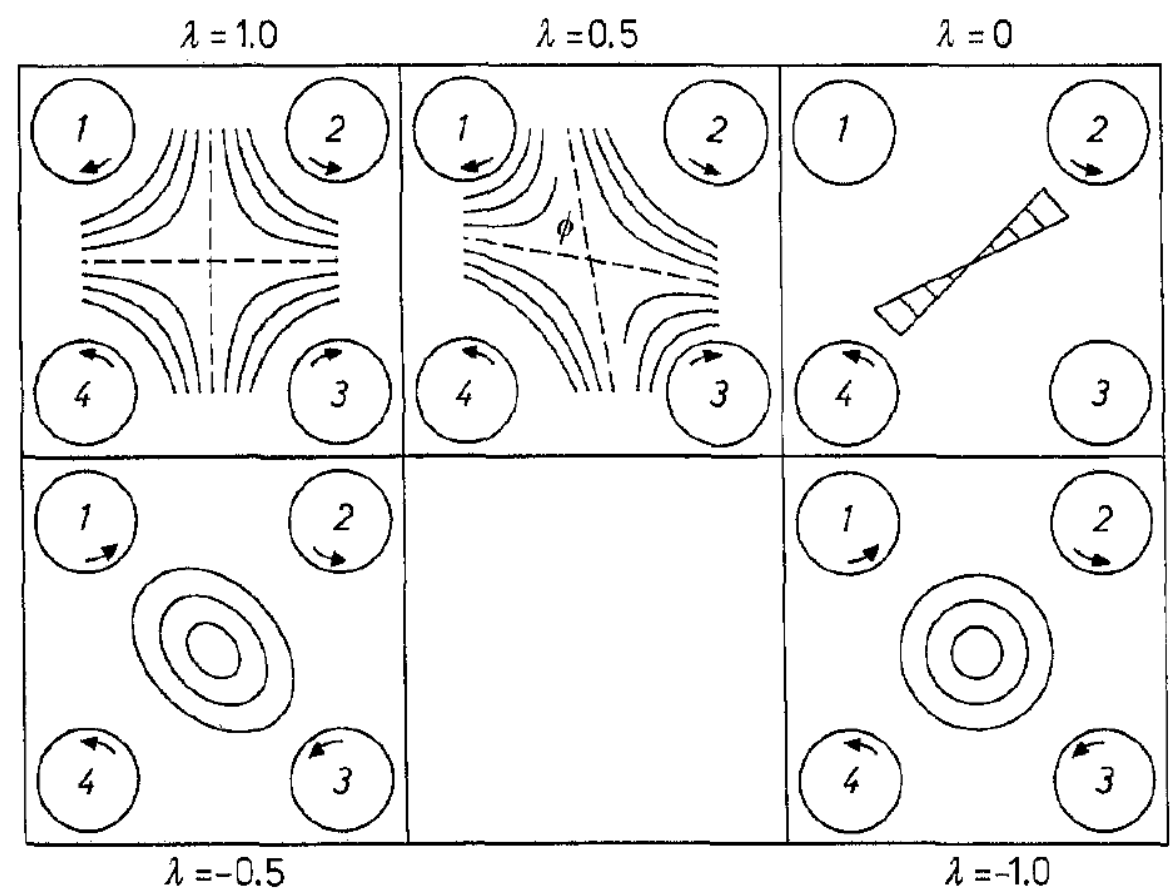

Figure 2.4: Streamlines of homogeneous two-dimensional flow [3]

An important parameter that arises is the Reynolds number of the rollers, which must suit all the flow configurations. The Reynolds number is defined in Equation 2-2, where $\omega_{r, \max } a$ is the modulus of the maximum angular speed among the pairs of rollers, $(2 b-a)$ is the gap width between rollers, and $\nu$ is the kinematic viscosity. Increasing the value of this parameter from small to intermediate values results in three-dimensional flow patterns in the four-roll mill.

$$
\begin{aligned}
& R e_{\text {rollers }}=\frac{\omega_{r, \max } a(2 b-a)}{\nu} \\
& \omega_{r, \max }=\max \left(\left|\omega_{r 1, r 3}\right|,\left|\omega_{r 2, r 4}\right|\right)
\end{aligned}
$$

\section{3}

\section{Literature review}




\subsection{1}

\section{Four-roll mill and drop deformation}

The four-roll mill was first mentioned in the literature by Taylor [13] as a mean to study the breakup of small droplets in a viscous straining motion, considering the interfacial tension effect. The so called "Four Roller" Apparatus was able to provide excellent approximations of linear flows between the rollers. His objective was to generate pure extensional flow.

Rumscheidt and Mason [14] focus their attention on expanding the previous work of Taylor. They investigated the deformation and breakup of fluid drops in both shear and hyperbolic flow. Their motivation was to examine the formation, stability, and rheology of emulsions and suspensions. At low deformations, their results were consistent with the work of Taylor. However, for large deformations in the shear flow, they notice that drop behavior is directly related to the viscosity ratio among the pair of fluids.

Giesekus [15] remarkably recognized that the variation of speed and direction of the rollers defined the magnitude of rate-of-strain. This discovery was an important step that had a powerful influence on the following works. Cox [16] presented a theoretical model that covers steady and unsteady flows in order to describe the shape of a fluid drop.

The capacity of generates two-dimensional linear flows by the four-roll mill permitted Fuller and Leal [17] to analyze birefringence in polymer solutions based on the criterion of weak and strong flows. It also contributed to the study of flow-induced crystallization in polymer melts by some other authors $[18,19]$.

The works of Bentley and Leal [20, 21] were a breakthrough. Besides the fact that all linear flows were considered (including time-dependent flows), an automated computer-controlled mechanism system was built to maintain droplets in the center position of the four-roll mill. Since the stagnation point is an unstable position, many previous works were restricted due to this issue.

Grace [22] analyzed a single drop deformation on both rotational and irrotational shear yields for high viscosity immiscible systems and their related application on the usage of static mixers on such systems. A experimental procedure was established to deal with static mixers on these types of flow.

Sherwood [23] included the effects of nonlinear terms of the imposed flow in his analysis of deformation of small emulsion droplets at low Reynolds number. This work considered the tip streaming phenomenon related to the ejection of small drops from the pointed ends.

Feng and Leal [24] used the four-roll mill to investigate the startup flow of dilute polymers solutions. A numeric simulation with finite element method 
was develop in order to solve the conservation of mass and momentum with the aid of FENE dumbell models, neglecting inertial effects.

Innings et al. [25] extended the original analysis by Taylor, including elliptic drops experiencing fast deformation. Dynamic and one-dimensional numerical simulations were implemented to accommodate the influence of continuous phase stress on the drop deformation, requiring the use of the dynamic Weber number. They conclude that the dynamic Weber number is not a function of the drop size, aspectio ratio and elongation rate.

Yang et al. [26] performed some experimental tests to interpret the coalescence between two equal-sized drops in a linear flow generated by a four-roll mill. In agreement with the available theoretical works, coalescence was very hard to accomplish in linear flows. A very low capillary number is necessary in order to provide a very subtle collision and reduce drop deformation.

Lagnado and Leal [3] analyzed the motion of a Newtonian fluid in the four-roll mill in order to discover Reynolds numbers of the rollers capable of providing three-dimensional effects. The configuration used in their experiments was associated with the flow-type $\lambda=1$ in order to obtain pure extensional flow near the center of the apparatus. For their specific apparatus dimensions, they noticed that until the Reynolds number of the rollers were around 37, they still obtained the two-dimensional pure extensional flow. Beyond this value, the appearance of swirling flow or vortex indicates the presence of three-dimensional flow.

\subsection{2}

\section{Flow classification}

It is essential to identify different rheological behavior that each flow pattern presents. The possibilities are in fact extensional flow, shear flow and rigid body motion. Astarita [27] enumerated a few properties that a flow classification criterion must contemplate, as listed in the sequence. The criterion can be either purely kinematic or one that additionally includes the mechanical behavior of the fluid.

(i) Local criterion - At each position, it should provide a flow classification.

(ii) Objective criterion - It must be invariant among different reference frames.

(iii) Generally applicable - It ought not be valid exclusively to a particular class of flow. 
All the previous works have failed to establish an acceptable criterion. The first criterion for flow classification was proposed by Astarita [28] years before. The flow-type parameters, including both the purely kinematic and the one which is not only purely kinematic, respectively were:

$$
\begin{gathered}
R_{I I D}=-\frac{\operatorname{tr} \boldsymbol{W}^{2}}{\operatorname{tr} \boldsymbol{D}^{\mathbf{2}}} \\
R_{I I E}=\Lambda^{2} \operatorname{tr}\left[\nabla \boldsymbol{u}^{T}\right]^{2}
\end{gathered}
$$

In these definitions, $\Lambda$ is the relaxation time, $\nabla \boldsymbol{u}=\left(\partial u_{j} / \partial x_{i}\right) \boldsymbol{e}_{i} \boldsymbol{e}_{j}$ is the velocity gradient, the velocity field is $u=u_{k} \boldsymbol{e}_{k}$, and, rate-of-deformation $\boldsymbol{D}=$ $\left(\nabla \boldsymbol{u}^{T}+\nabla \boldsymbol{u}\right) / 2$ and vorticity tensor $\boldsymbol{W}=\left(\nabla \boldsymbol{u}^{T}-\nabla \boldsymbol{u}\right) / 2$ are respectively the symmetric and antisymmetric parts of the transpose velocity gradient $\nabla \boldsymbol{u}^{T}$.

However, notice that both equations are not objective, as verified by Huilgol [29]. Since the work was also restricted to flow fields "between" viscometric and extensional, the criterion is not generally applicable.

Astarita and Marruci [30] display another criterion valid exclusively to motions with constant relative principal stretch history. Once again, it is not generally applicable. However, they were able to respect both the local and objective criterion. The flow index is defined with the aid of the tensor $\boldsymbol{N}$, which represents the unit of magnitude. This tensor is used to transform the deformation gradient $\boldsymbol{F}^{T}$ from time $t-s$ to the present time $t$ according to the following expression:

$$
\boldsymbol{F}^{T}=\boldsymbol{Q}(t-s)[\exp (-k s \boldsymbol{N})] \boldsymbol{Q}(t)
$$

where $\boldsymbol{Q}$ is a rotational time-dependent orthogonal second-order tensor and $k$ is a scalar parameter. The criterion proposed was:

$$
\begin{gathered}
R_{I I I D}=\operatorname{tr} \boldsymbol{N}^{2} \\
R_{I I I E}=\Lambda^{2} k^{2} \operatorname{tr} \boldsymbol{N}^{2}
\end{gathered}
$$

Tanner and Huilgol [31] presented a new criterion based on the classification of weak and strong flows. The criterion parameter is defined as:

$$
R_{I V D}=\max \left[\operatorname{Re}\left(\alpha_{k N}\right)\right]
$$

Basically, the expression of $R_{I V D}$ evaluates the maximum real part of the eigenvalues of $k \boldsymbol{N}$ and, if $R_{I V D}>0$, the flow is classified as strong. Otherwise, flow is denominated weak. Their study is in like manner restricted to motions 
with constant stretch history (MWCSH), since they did not look up to transient stress states. The analogous E-form was introduced by Tanner [32]:

$$
R_{I V E}=\max \left[\operatorname{Re}\left(\alpha_{\Lambda k N-\frac{1}{2} \boldsymbol{I}}\right)\right]
$$

Denn [33] pointed out that the analysis provided by Tanner and Huilgol was not very accurate for lubrication flows. Despite being shearing flows with a slight change in the cross section area, the definition classifies it as a strong flow. Denn indicates that it is also necessary to ensure that the largest element of $\nabla \boldsymbol{u}^{T}$ and the real part of an eigenvalue of $\nabla \boldsymbol{u}^{T}$ are of the same order of magnitude. Consequently, it suggests, even indirectly, a new criterion:

$$
R_{V D}=\max \left[\operatorname{Re}\left(\alpha_{\nabla \boldsymbol{u}^{T}}\right)\right]
$$

Since $\nabla \boldsymbol{u}^{T}$ is not objective, $R_{V D}$ is not sufficient to grant a complete flow classification criterion. In fact, none of the works managed to respect, at the same time, local, objective, and generally applicability criterion, ie (i), (ii) and (iii). As already mention, $R_{I I}$ and $R_{V}$ are not objective and $R_{I I I}$ and $R_{I V}$ are valid for particular cases only (MWCSH).

The first attempt to accommodate all the conditions was presented by Astarita [27]. The relative-rate-of-rotation tensor $\overline{\boldsymbol{W}}$ is obtained by:

$$
\bar{W}=W-\Omega
$$

$\boldsymbol{W}$ is the vorticity tensor and $\boldsymbol{\Omega}$ is the tensor which describes the rate of rotation from the eigenvalues of the rate-of deformation tensor $\boldsymbol{D}$. The main advantage of using $\overline{\boldsymbol{W}}$ is that, differently from $\boldsymbol{W}$ and $\Omega$, it is an objective parameter. The substantial time derivative of eigenvectors of $\boldsymbol{D}$ is related to $\boldsymbol{\Omega}$ through the following expression.

$$
\dot{\boldsymbol{e}}_{\boldsymbol{i}}=\boldsymbol{\Omega} \cdot \boldsymbol{e}_{\boldsymbol{i}}=\boldsymbol{\omega} \times \boldsymbol{e}_{\boldsymbol{i}}, \quad \mathrm{i}=1,2 \text { or } 3
$$

$\boldsymbol{\omega}$ is the angular velocity from the eigenvectors $\boldsymbol{e}_{\boldsymbol{i}}$. Notice that for the particular case in which there are two equals eigenvalues and one distinct, $\Omega$ is not defined. An approximation provided by Drouot and Lucius [34] is used to bypass this issue. For instance, if the eigenvalues 1,2 are equals, i.e., $\lambda_{1}=$ $\lambda_{2}$, it is necessary to add the condition that $\Omega_{12}=W_{12}$. With $\overline{\boldsymbol{W}}$ completely defined and being an objective, the criterion proposed is:

$$
\begin{gathered}
R_{D}=-\frac{\operatorname{tr} \overline{\boldsymbol{W}}^{2}}{\operatorname{tr} \boldsymbol{D}^{2}} \\
R_{E}=\Lambda\left(t r \overline{\boldsymbol{W}}^{2}+\operatorname{tr} \boldsymbol{D}^{2}\right)
\end{gathered}
$$


In relation to Astarita's flow classification criterion, besides taking account of property (ii), this criterion clearly provides different values and consequently different flow classification at each position and, therefore achieving property (i). Generally applicability (iii) is guaranteed since no restrictions of any type of flow were made.

Huilgol [35] showed three examples in each Astarita's criterion fails on being a successful flow criterion, i.e., respecting (i), (ii) and, (iii). First, he pointed out that for viscometric flows $R_{D}=1$. Unfortunately, other flows that are not viscometric also have $R_{D}=1$. Hence, there is not a one-to-one correspondence between $R_{D}$ and the flow type. Secondly, for the cases where two eigenvalues of $\boldsymbol{D}$ are equal and different for the third, there is no reason for using the approximation of Drouot and Lucius to define $\boldsymbol{\omega}$. The main problem is that when there are two eigenvalues that are very close to each other, the result of $R_{D}$ is not the same as if they were exactly the same due to the discontinuity.

Thompson and De Souza Mendes [36] came up with a new criterion that address these issues. They work with the velocity gradient as a measure of the reference frame attached to the eigenvectors of $\boldsymbol{D}$.

$$
\overline{\nabla \boldsymbol{u}^{T}}=\nabla \boldsymbol{u}^{T}-\Omega
$$

Huilgol examples allowed the perception of some important physical meanings. First, the rate of deformation of a material filament aligned orthogonally to the plane of rotation is not influenced by relative rotation. Secondly, the variation in intensity of persistence of straining experienced by a material filament it is a decrescent function of the difference between the largest and smallest rate of deformation rates that are present in the plane of relative rate of rotation.

The persistence-of-straining tensor and its respective intensity are:

$$
\begin{gathered}
\boldsymbol{P}=\boldsymbol{D} \overline{\boldsymbol{W}}-\overline{\boldsymbol{W}} \boldsymbol{D} \\
P=\sqrt{\frac{1}{2} \operatorname{tr}\left[(\boldsymbol{D} \overline{\boldsymbol{W}}-\overline{\boldsymbol{W}} \boldsymbol{D})^{2}\right]}
\end{gathered}
$$

A persistence-of-straining scalar parameter is specified to classify the flow. Thus, $\mathcal{R}=1, \mathcal{R}=0$, and $\mathcal{R}=\infty$ corresponds respectively to simple shear flow, extensional flow and rigid body motion. A one-to-one correspondence with this parameter and the flow classification is assured.

$$
\mathcal{R}=\frac{P}{\operatorname{tr}\left[\boldsymbol{D}^{2}\right]}
$$




\section{3}

\section{Model description}

\section{1}

\section{Domain and meshes}

In this work, two different domains were adopted. Both of the respective meshes were implemented with the aid of the software gmsh in order to run the desired simulations. According to the definition of the four-roll mill established in Section 2 and with Figure 2.3, the first domain will be referred from here on as the classic four-roll mill. This geometry is totally defined by the parameters $l, a$, and $b$, which respectively are the side of the tank, radius of cylinders and the gab between the cylinders.

The dimensions were chosen exactly as the work of Lagnado and Leal [3] and an irregular triangle mesh of $N_{c}=4246$ nodes was generated, as can be seen in Figure 3.1. The decision to keep the dimensions of a past experimental work on the literature is to provide an immediate comparison of results and also ensure that the flow will not present any kind of three-dimensional effect. Besides, the goal of this work is not to present ready-to-apply results. Rather, it aims at bringing new qualitative descriptions of different flows in the four-roll mill and analyze drop deformations in this context.

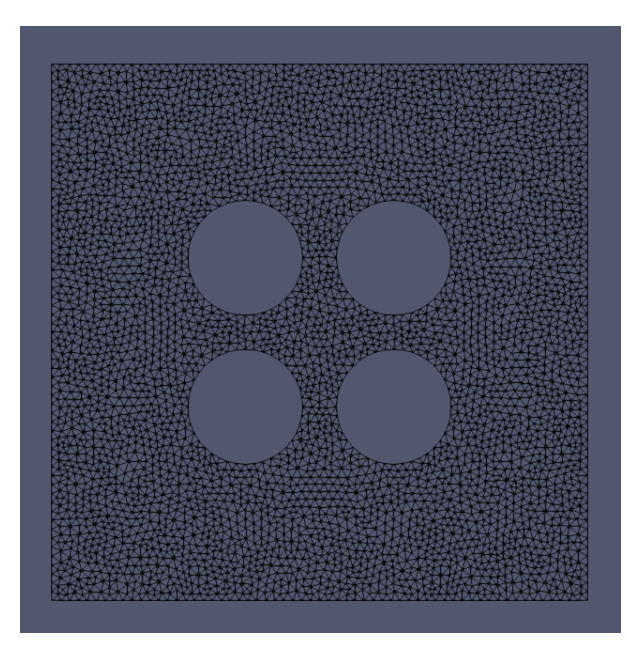

Figure 3.1: Classic four-roll mill mesh generated by gmsh. 
The second domain is a subtle variation from the classic four-roll mill domain. The objective of this new domain is to allow the imposition of an inlet constant velocity followed by a region of developed flow and consequently institute a clear flow direction. The only change is that the cylinders are immersed on an rectangular tank of length $6 l$ (the triple of the previous length). The height remains the same $(2 l)$. Thus, another irregular triangle mesh of $N_{e}=4912$ nodes was created.

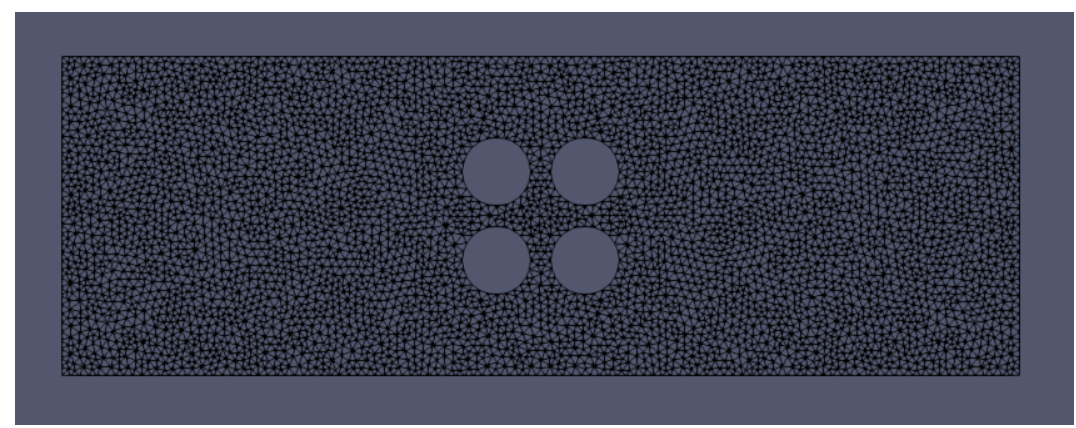

Figure 3.2: Expanded four-roll mill mesh generated by gmsh.

The dimensions related to both domains are exhibited on table 3.1. Furthermore, both meshes code files of gmsh, ie, 4RM_Mesh_Classic.geo and 4RM_Mesh_Expanded.geo, are available in Section A.

\begin{tabular}{cc}
\hline Parameters & Values \\
\hline$a(\mathrm{~m})$ & 0.01905 \\
$b(\mathrm{~m})$ & 0.02465 \\
$l(\mathrm{~m})$ & 0.26670
\end{tabular}

Table 3.1: Dimensions used in both domains.

\section{2}

\section{Modeling the flow}

The incompressible, bi-dimensional and isothermal flow of a Newtonian fluid in the four-roll mill at the steady-state is initially analyzed. The velocity field is defined as $\boldsymbol{u}=u_{x}(x, y) \boldsymbol{e}_{x}+u_{y}(x, y) \boldsymbol{e}_{y}$ where $\boldsymbol{e}_{x}$ and $\boldsymbol{e}_{y}$ are the basis vectors of the cartesian coordinate system. Therefore, physics is governed by the equations of conservation of mass and momentum, which respectively require that

$$
\begin{gathered}
\nabla \cdot \boldsymbol{u}=0 \\
\boldsymbol{u} \cdot \nabla \boldsymbol{u}-\nu \nabla^{2} \boldsymbol{u}=-\frac{1}{\rho} \nabla p
\end{gathered}
$$


where $\nabla=\partial / \partial \mathbf{x}$ is the standard gradient operator in physical space in which $\mathbf{x}$ is the position vector, $\rho$ is the specific mass, $\nu=\frac{\mu}{\rho}$ is the kinematic viscosity and $p$ is the pressure.

\section{3}

\section{Weak formulation}

This flow will be solved by the finite element method. Equations 3-1 and 3-2 represent the strong formulation. In order to solve this set of equations with the finite element method, it is necessary to rewrite the equations in the weak form. Therefore, the equations are multiplied by the respective weight functions $\boldsymbol{v}$ and $q$ and also integrated over the domain $\Psi$ :

$$
\begin{gathered}
\int_{\Psi}\left[(\boldsymbol{u} \cdot \nabla \boldsymbol{u}) \cdot \boldsymbol{v}-\nu\left(\nabla^{2} \boldsymbol{u}\right) \cdot \boldsymbol{v}\right] d \Psi=\int_{\Psi}\left[-\frac{1}{\rho} \nabla p \cdot \boldsymbol{v}\right] d \Psi \\
\int_{\Psi}(\nabla \cdot \boldsymbol{u}) q d \Psi=0
\end{gathered}
$$

The next aim is to eliminate second derivatives of velocity and any kind of derivatives of pressure, considering the element described in the sequence. A vector calculus identity was adopted to vanish the velocity laplacian.

$$
\int_{\Psi}\left(\nabla^{2} \boldsymbol{u}\right) \cdot \boldsymbol{v} d \Psi=-\int_{\Psi} \nabla \boldsymbol{u}: \nabla \boldsymbol{v} d \Psi+\int_{\Psi} \nabla \cdot(\nabla \boldsymbol{u} \cdot \boldsymbol{v}) d \Psi
$$

Using the divergence theorem for the equation above:

$$
\int_{\Psi}\left(\nabla^{2} \boldsymbol{u}\right) \cdot \boldsymbol{v} d \Psi=-\int_{\Psi} \nabla \boldsymbol{u}: \nabla \boldsymbol{v} d \Psi+\oint_{\Gamma} \boldsymbol{n} \cdot(\nabla \boldsymbol{u} \cdot \boldsymbol{v}) d \Gamma
$$

On the other hand, integration by parts is the key to replace the gradient pressure.

$$
\int_{\Psi} \nabla p \cdot \boldsymbol{v} d \Psi=\oint_{\Gamma} p(\boldsymbol{n} \cdot \boldsymbol{v}) d \Gamma-\int_{\Psi} p \nabla \cdot \boldsymbol{v} d \Psi
$$

Substituting the respective terms on equation 3-3:

$$
\begin{array}{r}
\int_{\Psi}(\boldsymbol{u} \cdot \nabla \boldsymbol{u}) \cdot \boldsymbol{v} d \Psi+\nu\left[\int_{\Psi} \nabla \boldsymbol{u}: \nabla \boldsymbol{v} d \Psi-\oint_{\Gamma} \boldsymbol{n} \cdot(\nabla \boldsymbol{u} \cdot \boldsymbol{v}) d \Gamma\right]= \\
\frac{1}{\rho}\left[-\oint_{\Gamma} p(\boldsymbol{n} \cdot \boldsymbol{v}) d \Gamma+\int_{\Psi} p \nabla \cdot \boldsymbol{v} d \Psi\right]
\end{array}
$$

\section{4}

\section{Element}

In order to increase the solver speed and facilitate convergence, a mixed element is used. The Taylor-Hood element is composed by two standard 
Lagrange elements, one for the velocity components, which are $u_{x}$ and $u_{y}$, and one for the pressure calculation $p$. Therefore, the coupled solution between velocity and pressure is defined by the $P_{r}{ }^{-} \Lambda^{k}$ family, where $r=1,2$ and $k=0$.

Hence, the pressure is continuous and varies linearly with the position $\boldsymbol{x}$ over each cell on the mesh. However, the degree of the basis function related to the velocity element is quadratic. Pressure is evaluated at each cell vertex, while velocities are evaluated also at middle of cell facet.

This element is commonly used to solve Navier-Stokes equations in the literature. The stability and accuracy of this element were previous analyzed by Bercovier and Pironneau [37], Lee and Li [38] and Guzmán and Sánchez [39].

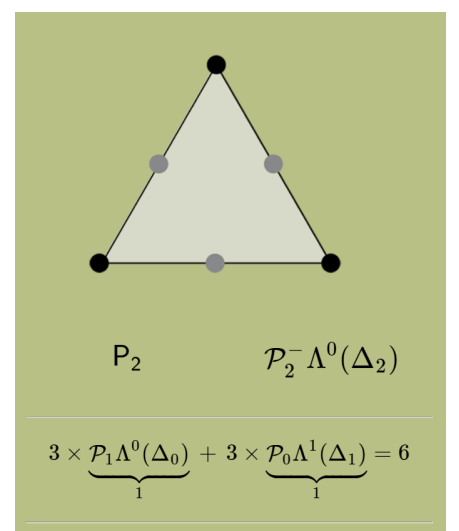

Figure 3.3: Mixed lagrange element

\section{5}

\section{Boundary conditions}

Each domain has its own boundary conditions. On both of them, the borders are called "West", "North", "East", and "South". On the classic four-roll mill, every border corresponds to an impermeable slippery wall. Therefore, at each border, the normal velocity to the current border is null and the derivative of the tangent velocity (to the border) in the normal direction (to the border) is also null. The tangent velocity of the cylinder $i$ is naturally $\boldsymbol{u} \cdot \boldsymbol{t}=\omega_{r, i} a$, with $i$ varying from 1 to 4 . Since this flow is not driven by a pressure gradient, it is set that the pressure is null for every border. 


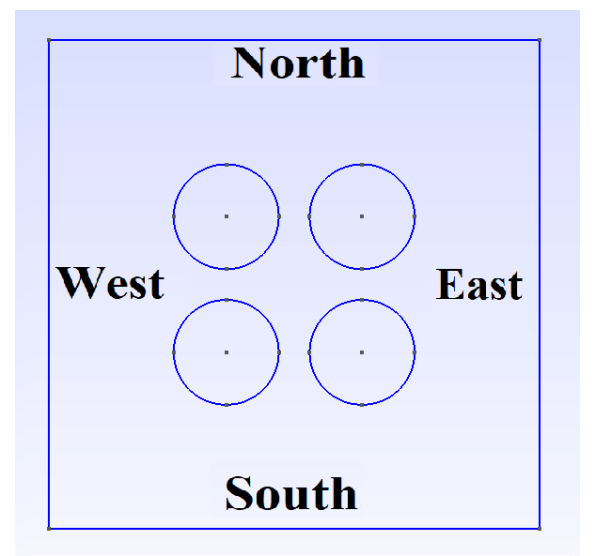

Figure 3.4: Borders of the classic four-roll mill.

\begin{tabular}{ccccc}
\hline West & North & East & South & Cylinders \\
\hline $\mathbf{n}=(-1,0)$ & $\mathbf{n}=(0,1)$ & $\mathbf{n}=(1,0)$ & $\mathbf{n}=(0,-1)$ & $\mathbf{u} \cdot \mathbf{t}= \pm \omega_{r, i} a$ \\
$u_{x}=0$ & $u_{y}=0$ & $u_{x}=0$ & $u_{y}=0$ & \\
$\frac{\partial u_{y}}{\partial x}=0$ & $\frac{\partial u_{x}}{\partial y}=0$ & $\frac{\partial u_{y}}{\partial x}=0$ & $\frac{\partial u_{x}}{\partial y}=0$ & \\
$p_{W}=0$ & $p_{N}=0$ & $p_{E}=0$ & $p_{S}=0$ & \\
\hline
\end{tabular}

Table 3.2: Boundary conditions in the classic four-roll mill.

The expanded four-roll mill work with different boundary conditions. In the West border, which is the inlet, a constant velocity is imposed in the $\boldsymbol{e}_{x}$ direction. It is worth mentioning that this non-null inlet velocity is essential to ensure the flow direction from West to East. The borders North and South correspond to impermeable slippery walls. Thus, it is valid to claim that the derivative of the tangent velocity in the normal direction is also null. Lastly, the condition of developed flow at the outlet (East) is used. Therefore, the velocity field at this border does not vary in the $x$ direction. In this case, since there is a imposed velocity, it is important to define a level of pressure. Thus, it was adopted that $p_{E}=0$ in the "East" border. 


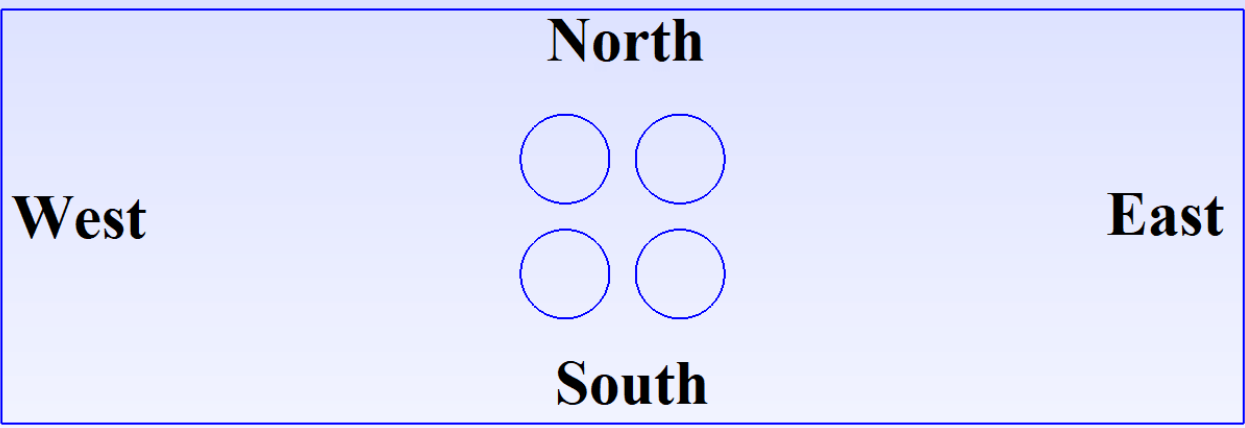

Figure 3.5: Borders of the expanded four-roll mill.

\begin{tabular}{ccccc}
\hline West & North & East & South & Cylinders \\
\hline $\mathbf{n}=(-1,0)$ & $\mathbf{n}=(0,1)$ & $\mathbf{n}=(1,0)$ & $\mathbf{n}=(0,-1)$ & $\mathbf{u} \cdot \mathbf{t}= \pm \omega_{r, i} a$ \\
$\boldsymbol{u}=U_{\infty} \boldsymbol{e}_{x}$ & $u_{y}=0$ & $\boldsymbol{n} \cdot \nabla \boldsymbol{u}=0$ & $u_{y}=0$ & \\
& $\frac{\partial u_{x}}{\partial y}=0$ & $p_{E}=0$ & $\frac{\partial u_{x}}{\partial y}=0$ & \\
\hline
\end{tabular}

Table 3.3: Boundary conditions in the expanded four-roll mill.

In both meshes, the boundary conditions result in:

$$
\begin{array}{r}
\oint_{\Gamma} \boldsymbol{n} \cdot(\nabla \boldsymbol{u} \cdot \boldsymbol{v}) d \Gamma=\oint_{N} \boldsymbol{n} \cdot(\nabla \boldsymbol{u} \cdot \boldsymbol{v}) d \Gamma+\oint_{W} \boldsymbol{n} \cdot(\nabla \boldsymbol{u} \cdot \boldsymbol{v}) d \Gamma+ \\
\oint_{S} \boldsymbol{n} \cdot(\nabla \boldsymbol{u} \cdot \boldsymbol{v}) d \Gamma+\oint_{E} \boldsymbol{n} \cdot(\nabla \boldsymbol{u} \cdot \boldsymbol{v}) d \Gamma=0+0+0+0=0
\end{array}
$$

The term that involves the pressure in the borders are also null. For the "West", "North" and "South" borders, a essential boundary condition is imposed for the velocity. Therefore, the residual term is abandoned and replaced by this condition. In its turn, for the "East" border it is already imposed that $p_{E}=0$.

$$
\begin{array}{r}
\oint_{\Gamma} p(\boldsymbol{n} \cdot \boldsymbol{v}) d \Gamma=\oint_{W} p_{W}(\boldsymbol{n} \cdot \boldsymbol{v}) d \Gamma+\oint_{E} p_{E}(\boldsymbol{n} \cdot \boldsymbol{v}) d \Gamma \\
+\oint_{S} p_{S}(\boldsymbol{n} \cdot \boldsymbol{v}) d \Gamma+\oint_{N} p_{N}(\boldsymbol{n} \cdot \boldsymbol{v}) d \Gamma=0+0+0+0=0
\end{array}
$$

Replacing the expressions above in equation (3-8), the weak formulation related to the conservation of momentum can be written as:

$$
\int_{\Psi}(\boldsymbol{u} \cdot \nabla \boldsymbol{u}) \cdot \boldsymbol{v} d \Psi+\nu \int_{\Psi} \nabla \boldsymbol{u}: \nabla \boldsymbol{v} d \Psi-\frac{1}{\rho} \int_{\Psi} p \nabla \cdot \boldsymbol{v} d \Psi=0
$$

The equation of mass conservation remains almost the same, being multiplied by the scalar weight function $q$ and integrated over the domain $\Psi$ : 


$$
\int_{\Psi}(\nabla \cdot \boldsymbol{u}) q d \Psi=0
$$

The nonlinear variational problem was solve with the Newton-Raphson method according to FeniCs project libraries. Despite being a pretty robust method, as literature suggest [40,41,42], it heavily relies on a reasonable initial guess to converge. In the code, the solver was set with both absolute and relative tolerances of $10^{-8}$ and a number maximum of iterations of 100 .

\section{6}

\section{Persistence of straining}

The flow classification criterion proposed by Thompson and de Souza Mendes [36] is employed as a part of the post-processing scheme. The advantages of this choice is related to obtain a local classification, independent from the frame of reference and not restricted to a set of particular flows.

The pesistence-of-straining tensor $\boldsymbol{P}$ is defined as:

$$
\boldsymbol{P}=\boldsymbol{D} \cdot \overline{\boldsymbol{W}}-\overline{\boldsymbol{W}} \cdot \boldsymbol{D}
$$

where $\boldsymbol{D}=\frac{1}{2}\left(\nabla \boldsymbol{u}+\nabla \boldsymbol{u}^{T}\right)$ is rate-of-deformation tensor, $\boldsymbol{W}=$ $\frac{1}{2}\left(\nabla \boldsymbol{u}-\nabla \boldsymbol{u}^{T}\right)$ is the vorticity tensor, $\bar{W}=\boldsymbol{W}-\boldsymbol{\Omega}$ is the relativity vorticity tensor and, $\Omega$ is the tensor that provides the rates of rotation from the eigenvectors of $\boldsymbol{D}$.

Notice that $\boldsymbol{P}$ may be rewritten as:

$$
\boldsymbol{P}=(\boldsymbol{D} \cdot \boldsymbol{W}-\boldsymbol{W} \cdot \boldsymbol{D})-(\boldsymbol{D} \cdot \boldsymbol{\Omega}-\boldsymbol{\Omega} \cdot \boldsymbol{D})
$$

As long as the velocity field is obtained, $\boldsymbol{D}$ e $\boldsymbol{W}$ are easily evaluated. The difficulty resides in achieving $\boldsymbol{\Omega}$. For this purpose, $\boldsymbol{D}$ is initially written in its own eigenvectors coordinate system, and hence in a diagonal matrix form.

$$
\boldsymbol{D}=\sum_{i=1}^{3} \xi_{i} \hat{e}_{i} \hat{e}_{i}
$$

where $\xi_{1} \geq \xi_{2} \geq \xi_{3}$ are the eigenvalues of $\boldsymbol{D}$ and $\hat{e_{1}}, \hat{e_{2}}$, and $\hat{e_{3}}$ are their respective eigenvectors.

In sequence, the material time derivative of $\boldsymbol{D}$ is displayed:

$$
\dot{\boldsymbol{D}}=\sum_{i=1}^{3} \dot{\xi}_{i} \hat{e}_{i} \hat{e}_{i}+\sum_{i=1}^{3} \xi_{i} \dot{\hat{e}}_{i} \hat{e}_{i}+\sum_{i=1}^{3} \xi_{i} \hat{e}_{i} \dot{\hat{e}}_{i}
$$

According to the definition of $\boldsymbol{\Omega}$, it follows that $\dot{\hat{e}}_{i}=\boldsymbol{\Omega} \hat{e}_{i}$. Therefore: 


$$
\begin{aligned}
\dot{\boldsymbol{D}}=\sum_{i=1}^{3} \dot{\xi}_{i} \hat{e}_{i} \hat{e}_{i}+\sum_{i=1}^{3} \xi_{i} \boldsymbol{\Omega} \hat{e}_{i} \hat{e}_{i}+\sum_{i=1}^{3} \xi_{i} \hat{e}_{i} \boldsymbol{\Omega} \hat{e}_{i} & \\
\sum_{i=1}^{3} \dot{\xi}_{i} \hat{e}_{i} \hat{e}_{i}+\boldsymbol{\Omega}\left(\sum_{i=1}^{3} \xi_{i} \hat{e}_{i} \hat{e}_{i}\right) & +\left(\sum_{i=1}^{3} \xi_{i} \hat{e}_{i} \hat{e}_{i}\right) \boldsymbol{\Omega}^{T}= \\
& \sum_{i=1}^{3} \dot{\xi}_{i} \hat{e}_{i} \hat{e}_{i}+\boldsymbol{\Omega} \cdot \boldsymbol{D}+\boldsymbol{D} \cdot \boldsymbol{\Omega}^{T}
\end{aligned}
$$

Since $\boldsymbol{\Omega}$ is antisymmetric, i.e. $\boldsymbol{\Omega}^{T}=-\boldsymbol{\Omega}$, the previous expression may be rewritten as:

$$
\dot{\boldsymbol{D}}=\sum_{i=1}^{3} \dot{\xi}_{i} \hat{e}_{i} \hat{e}_{i}+\boldsymbol{\Omega} \cdot \boldsymbol{D}-\boldsymbol{D} \cdot \boldsymbol{\Omega}
$$

Combining equations (3-12) and (3-15):

$$
\boldsymbol{P}=\boldsymbol{D} \cdot \boldsymbol{W}-\boldsymbol{W} \cdot \boldsymbol{D}+\left[\dot{\boldsymbol{D}}-\sum_{i=1}^{3} \dot{\xi}_{i} \hat{e}_{i} \hat{e}_{i}\right]
$$

It is important to recognize that the eigenvectors $\hat{e}_{i}$ vary with the position and time. $\boldsymbol{u}, \nabla \boldsymbol{u}, \boldsymbol{D}$, and $\boldsymbol{W}$ are typically written on a system coordinate which basis is $\left\{\hat{i}_{i}\right\}$. Therefore, the final objective is to write $\boldsymbol{P}$ in terms of the basis $\left\{\hat{i}_{i}\right\} . \boldsymbol{Q}$ is the rotation tensor which transforms $\left\{\hat{i}_{i}\right\}$ in $\left\{\hat{e}_{i}\right\}$. Thus:

$$
\left\{\dot{\boldsymbol{D}}-\sum_{i=1}^{3} \dot{\xi}_{i} \hat{e}_{i} \hat{e}_{i}\right\}_{\left\{\hat{i}_{i}\right\}}=\left\{\boldsymbol{Q}^{T}\right\}\left\{\dot{\boldsymbol{D}}-\sum_{i=1}^{3} \dot{\xi}_{i} \hat{e}_{i} \hat{e}_{i}\right\} \underbrace{}_{\left\{\hat{e}_{i}\right\}}\{\boldsymbol{Q}\}=\dot{\boldsymbol{D}}-\boldsymbol{Q}^{\boldsymbol{T}} \sum_{i=1}^{3} \dot{\lambda}_{i} \hat{e}_{i} \hat{e}_{i} \boldsymbol{Q}
$$

Combining equations (3-16) and (3-17), and considering that $\dot{\boldsymbol{D}}=$ $\frac{\partial \boldsymbol{D}}{\partial t}+\boldsymbol{u} \cdot \nabla \boldsymbol{D}, \boldsymbol{P}$ is finally written in terms of $\left\{\hat{i}_{i}\right\}$.

$$
\boldsymbol{P}=\boldsymbol{D} \cdot \boldsymbol{W}-\boldsymbol{W} \cdot \boldsymbol{D}+\frac{\partial \boldsymbol{D}}{\partial t}+\boldsymbol{u} \cdot \nabla \boldsymbol{D}-\boldsymbol{Q}^{\boldsymbol{T}} \sum_{i=1}^{3} \dot{\lambda}_{i} \hat{e}_{i} \hat{e}_{i} \boldsymbol{Q}
$$

The flow-type parameter $\mathcal{R}$ is readily obtained.

$$
\mathcal{R}=\frac{P}{\operatorname{tr}\left[\boldsymbol{D}^{2}\right]}
$$

In order to obtain values in a finite range, the following normalization is proposed:

$$
\mathcal{R}^{*}=\frac{1-\mathcal{R}}{1+\mathcal{R}}
$$

Pure extensional flows correspond to the maximum intensity to persistence-of-straining, $\mathcal{R}^{*}=1$. The viscometric flow implies on the value 
of persistence-of-straining $\mathcal{R}^{*}=0$. The motion of rigid body is associated to $\mathcal{R}^{*}=-1$. Intermediate values represent transition regions that contains the presence of both behaviors. This parameter does not quantify which behavior is predominant. Every single mesh node will have its own flow classification and thus an $\mathcal{R}^{*}$ field is going to be available. Notice that for these specific $2 \mathrm{D}$ application analyzed in this work: $\xi_{3}=0$.

\section{7}

\section{Microelement's deformation}

As a second post-processing scheme, the behavior of microelements is analyzed on different flows generated by the four-roll mill. It represents the dispersed phase of the emulsion. The microelement and its respective microstructure is characterized by a passive vector $\boldsymbol{R}$. Consequently, it does not influence the velocity field. However, despite following a predetermined pathline, the microelement does not deform as another element from the continuous phase would do at the same pathline. In order to predict the microelement deformation, the work of Olbricht is required [43]. The form and magnitude of the velocity gradient tensor are evaluated to provide the strength of the flow. The deformation of the microelement is based on the classical linear stability analysis. Another important limitation of this analysis is to consider only simple flows with constant stretch history.

As already mentioned, the vector follows its path according to the flow velocity field:

$$
\boldsymbol{x}=\int \boldsymbol{u}(\boldsymbol{x}) d t
$$

The deformation of the microelement is dictated by:

$$
\dot{\boldsymbol{R}}=\boldsymbol{W} \cdot \boldsymbol{R}+G\left[\boldsymbol{D} \cdot \boldsymbol{R}-\frac{F}{F+1}(\boldsymbol{r} \cdot \boldsymbol{D} \cdot \boldsymbol{r}) \boldsymbol{R}\right]-\frac{\alpha}{F+1} \boldsymbol{R}
$$

where $\boldsymbol{r}=\frac{\boldsymbol{R}}{R}$ and $R \equiv|\boldsymbol{R}|$. The coefficients $G, \alpha$, and $\mathrm{F}$ are parameters which depend on the properties of both fluids, i.e., continuous and dispersed phases.

For convenience, equation (3-22) is rewritten in a new format:

$$
\dot{\boldsymbol{R}}=\left(\boldsymbol{W}+\boldsymbol{D}_{\text {mod }}\right) \cdot \boldsymbol{R}
$$

Therefore, $\boldsymbol{D}_{\text {mod }}$ is automatically defined as:

$$
\boldsymbol{D}_{\text {mod }}=G\left[\boldsymbol{D}-\frac{F}{F+1}(\boldsymbol{r} \cdot \boldsymbol{D} \cdot \boldsymbol{r}) \boldsymbol{I}\right]-\frac{\alpha}{F+1} \boldsymbol{I}
$$


The combination of the parameters $G, \alpha$ e $F$ describes the microelement and reflects the physical meaning of its microstructure. Notice the particular case in which $G=1, \alpha=0$, and $F=0$ implies that $\boldsymbol{D}_{\text {mod }}=\boldsymbol{D}$ and therefore the microelement behaves exactly the sames as another fluid element from the continuous phase. In the vast majority of cases, rigid and axisymmetric particles contain $G<1$. In order to consider a model with internal viscosity, commonly known in the literature as elastic dumbbell, non-null values of $\mathrm{F}$ are necessary.

Another advantage of bringing the work of Olbricht is the possibility of immediately providing a new flow classification depending on which phase is present on each node at a specific time. In order to display a flow classification for the dispersed phase, it is necessary to repeat the procedure presented in Section 3.6, using $\boldsymbol{D}_{\text {mod }}$ instead of $\boldsymbol{D}$. Thus, the field $\mathcal{R}^{*}{ }_{\text {mod }}$ is obtained, which at every node provides a new flow classification if the current position is, in fact, occupied by the dispersed phase. Along these lines, it is possible to visualize completely different scenarios of deformation on the same mesh position, depending if the fluid is from dispersed or continuous phase.

$$
\begin{gathered}
\mathcal{R}_{\text {mod }}=\frac{P}{\operatorname{tr}\left[\boldsymbol{D}_{\text {mod }}^{2}\right]} \\
\mathcal{R}_{\text {mod }}{ }^{*}=\frac{1-\mathcal{R}_{\text {mod }}}{1+\mathcal{R}_{\text {mod }}}
\end{gathered}
$$

To deal with these equations, a simple numerical procedure is sufficient to accomplish the goal of the post-processing scheme. Given an initial position $\boldsymbol{x}^{1}$ at time $t=0$, the pathline of the vector that represents a microelement of the dispersed phased is determined by the Euler method:

$$
\begin{gathered}
x^{n+1}=x^{n}+\frac{\Delta t}{2}\left[u_{x}^{n}+u_{x}^{n+1}\right], n=1, \ldots, N-1 \\
y^{n+1}=y^{n}+\frac{\Delta t}{2}\left[y_{x}^{n}+y_{x}^{n+1}\right], n=1, \ldots, N-1
\end{gathered}
$$

On other hand, the initial values of the vector $\boldsymbol{R}$ in the directions $\boldsymbol{e}_{x}$ and $\boldsymbol{e}_{y}$ must be consistent with the fact that the theory predict small deformations $(|\boldsymbol{R}|<<1)$. Given an initial value $R^{1}$ and consequently the components associated a initial inclination $\theta^{1}$, ie $R_{x}{ }^{1}=R^{1} \cos \left(\theta^{1}\right)$ and $R_{y}{ }^{1}=R^{1} \sin \left(\theta^{1}\right)$. Thus, the temporal evolution of $R_{x}$ and $R_{y}$ by the Crank-Nicolson method:

$$
\begin{array}{r}
R_{x}{ }^{n+1}=R_{x}{ }^{n}+\frac{\Delta t}{2}\left\{\left(W_{11}+G\left[D_{11}-\frac{F}{F+1}(\boldsymbol{r} \cdot \boldsymbol{D} \cdot \boldsymbol{r})\right]-\frac{\alpha}{F+1}\right)\left(R_{x}{ }^{n}+R_{x}{ }^{n+1}\right)+\right. \\
\left.\left(W_{12}+G_{12}\right)\left(R_{y}{ }^{n}+R_{y}{ }^{n+1}\right)\right\}
\end{array}
$$




$$
\begin{gathered}
R_{y}{ }^{n+1}=R_{y}{ }^{n}+\frac{\Delta t}{2}\left\{\left(W_{22}+G\left[D_{22}-\frac{F}{F+1}(\boldsymbol{r} \cdot \boldsymbol{D} \cdot \boldsymbol{r})\right]-\frac{\alpha}{F+1}\right)\left(R_{y}{ }^{n}+R_{y}{ }^{n+1}\right)+\right. \\
\left.\left(W_{21}+G_{21}\right)\left(R_{x}{ }^{n}+R_{x}{ }^{n+1}\right)\right\}
\end{gathered}
$$

The numeric procedure is repeated while the position of the vector is still inside the domain, i.e., $-l \leq x^{n+1} \leq l$ and $-3 l \leq y^{n+1} \leq 3 l$, and throughout the time that the size of the vector does not reach any limited values associated with its deformation. As a consequence, maximum and minimum relative values of deformation interrupt the code, as well as the vector leaving the domain, and therefore the number of steps $N-1$ is determined. The modulus of the vector, at each time step, is naturally obtained as $R=\sqrt{R_{x}{ }^{2}+R_{y}{ }^{2}}$ and it indicates the size of the microelement.

\section{8}

\section{Non-dimensionalization}

Aiming to obtain non-dimensional results, the first step is to define the characteristic parameters. The characteristic length, velocity and time are respectively $x_{c}=a, u_{c}=\omega_{r, \max } a$, and $t_{c}=1 / \omega_{r, \max }$. Consequently, the dimensionless parameters become $x^{*}=x / a, y^{*}=y / a, u^{*}=u /\left(\omega_{r, \max } a\right)$, and $t^{*}=\omega_{r, \max } t$. Analogously with the Reynolds number of the rollers defined on equation 2-2, an entrance Reynolds number is evaluated by equation 3-30. Therefore, the ratio between both of the Reynolds number is reduced to a ratio of velocities.

$$
R e_{e n t}=\frac{U_{\infty}(2 b-a)}{\nu}
$$




\section{4 \\ Results}

This chapter presents the results of the numerical simulations on the four-roll mill. First, the classic four-roll mill is considered in order to compare its results with the literature. Thus, $R e_{e n t}=0$ is fixed in this case. In the sequence, the expanded four-roll mill provides additional analysis about the microelement deformation. This time, non-null inlet velocities are analyzed.

The fluid that is originally used on equations 3-9 and 3-10 is a Newtonian oil with viscosity $\mu=34 \times 10^{-3} \mathrm{~Pa} \cdot \mathrm{s}$ and density $\rho=887.2 \mathrm{~kg} / \mathrm{m}^{3}$. Both of these parameters were obtained with a rheological characterization by Naccache, J. P. A. [44] at the temperature of $T=40^{\circ} \mathrm{C}$. The non-dimensional parameters, based on Table 3.1 and the non-dimensionalization scheme presented on Chapter 3, are displayed in Table 4.1. All the results are presented in dimensionless form.

\begin{tabular}{cc}
\hline Parameters & Values \\
\hline$b^{*}$ & 1.294 \\
$l^{*}$ & 14 \\
$R e_{\text {rollers }}$ & 20
\end{tabular}

Table 4.1: Dimensionless parameters used in the four-roll mill simulations.

\section{1}

\section{Classic four-roll mill}

As already mentioned, $R e_{e n t}=0$ for this mesh configuration. This section covers the results of non-dimensional velocity fields, streamlines, normalized persistence-of-straining parameter field, and modified normalized persistenceof-straining parameter field for the classic four-roll mill. Different flow configurations are investigated.

\subsection{1}

\section{Velocity fields}

The velocity fields obtained with different flow configurations are presented in Figure 4.1. They represent a standard and expected output, according to the literature related with the four-roll mill. Notice that the origin is a 
stagnation point for every single flow configuration. Figure $4.1 \mathrm{i}$ ) is the only case $(\lambda=1)$ capable of producing velocities considerably higher than the tangent velocity on the cylinders borders, specifically in the region between rollers. It also brings the particular presence of symmetry in relation of axis $x^{*}$ and $y^{*}$. The configuration presented in Figure $\left.4.1 \mathrm{ii}\right)(\lambda=0.5)$ is a small variation from the plot $4.1 \mathrm{i}$ ) in which the angular velocity of rollers pair 1,3 is half of the pair 2,4 . In figure $4.1 \mathrm{iii}$ ), the pair of cylinders 1,3 is static, i.e. $\lambda=0$, while in Figure $4.1 \mathrm{iv}$ ) the angular velocity of rollers pair 1,3 is half of the pair 2,4 one more time with $\lambda=-0.5$. What differs the cases ii) and iv) are the directions of the angular speed of the rollers. In a few cases, such as 4.1 ii), 4.1 iii), and $4.1 \mathrm{iv})$, the tangent velocity of pair 2,4 is the maximum velocity of the field and the presence of lower velocities results in external pathlines of fluid particles around the cylinders. The velocity field from the configuration $\lambda=-1$, which is presented in Figure $4.1 \mathrm{v}$ ), is extremely similar from the flow in which the four cylinders are replaced by a single cylinder rotating counterclockwise with the same angular velocity.
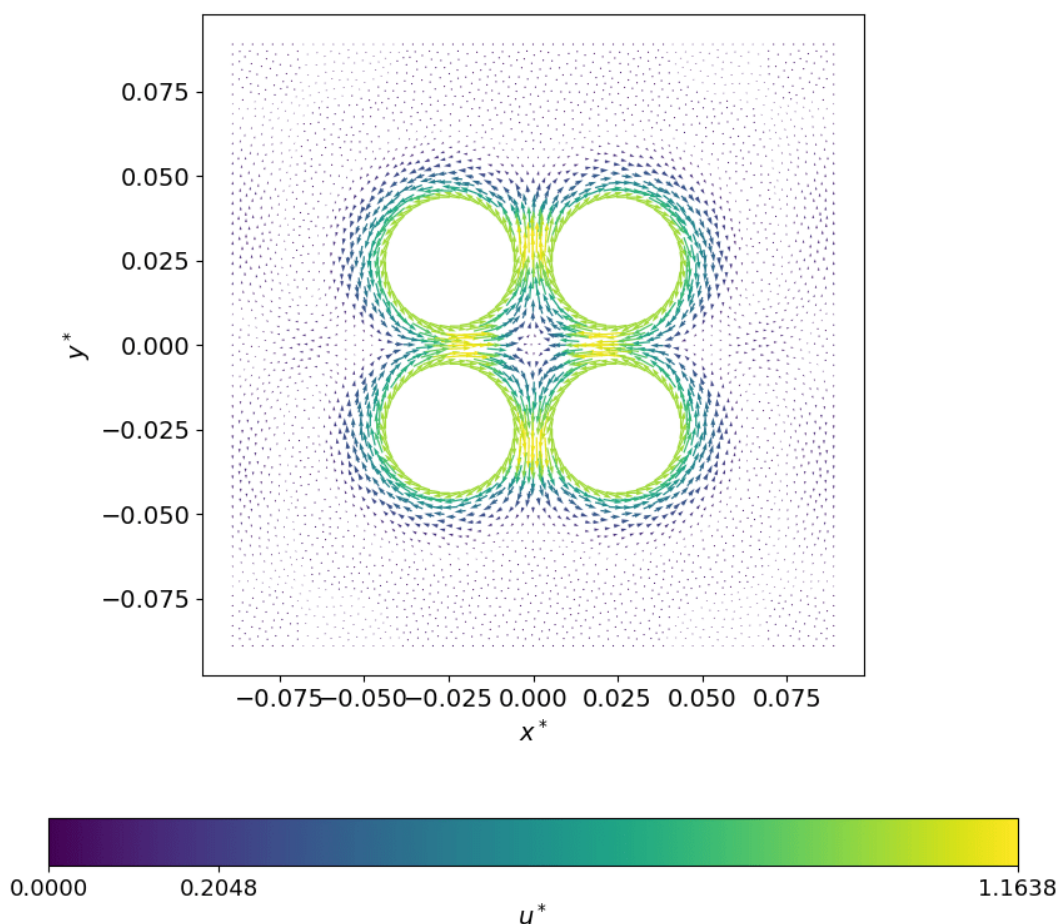

i) $\lambda=1$ 

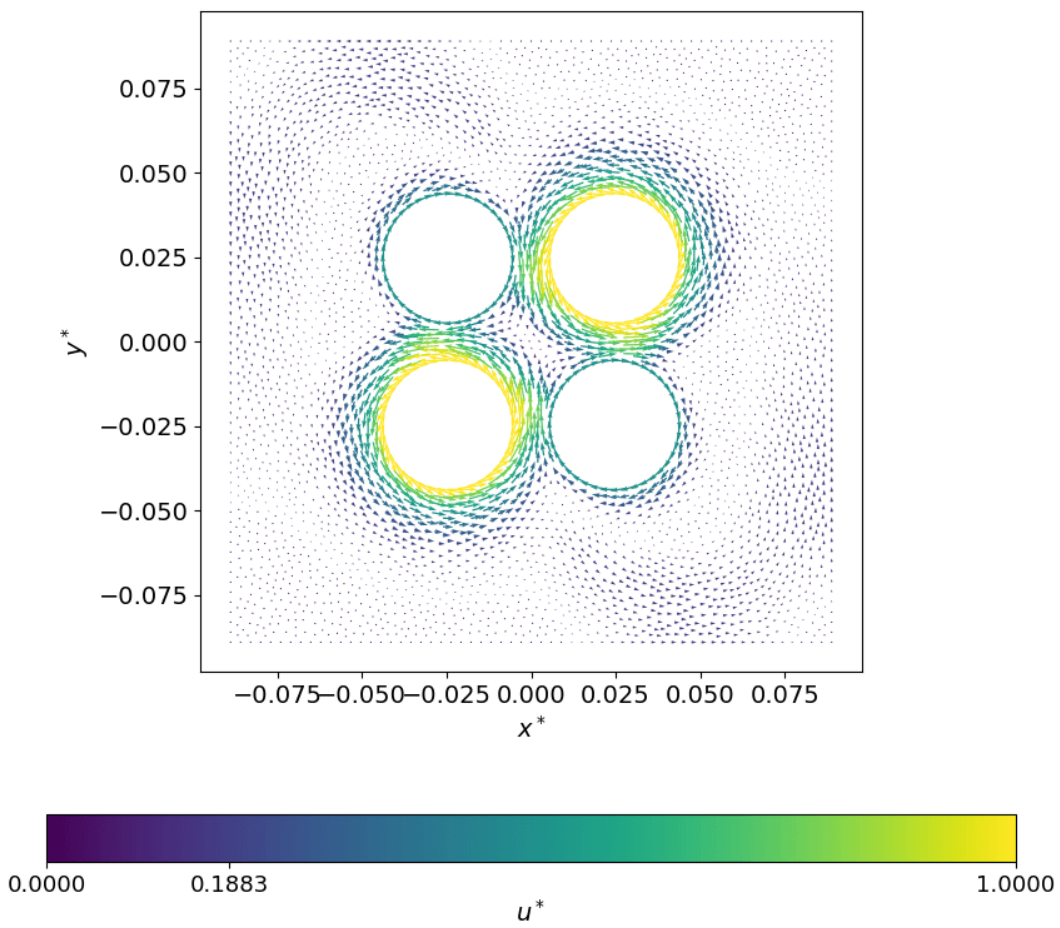

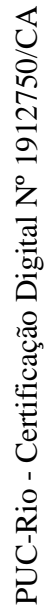

ii) $\lambda=0.5$

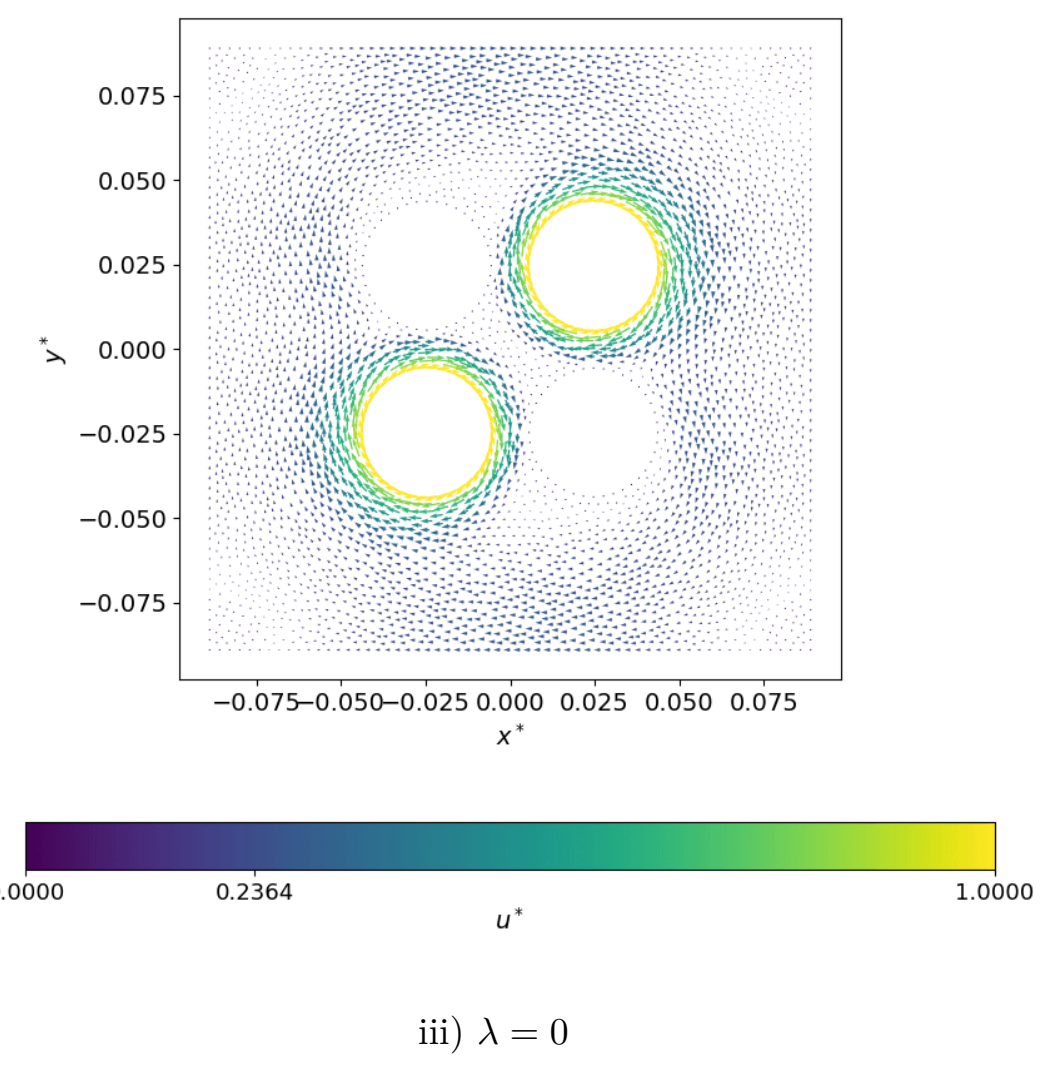



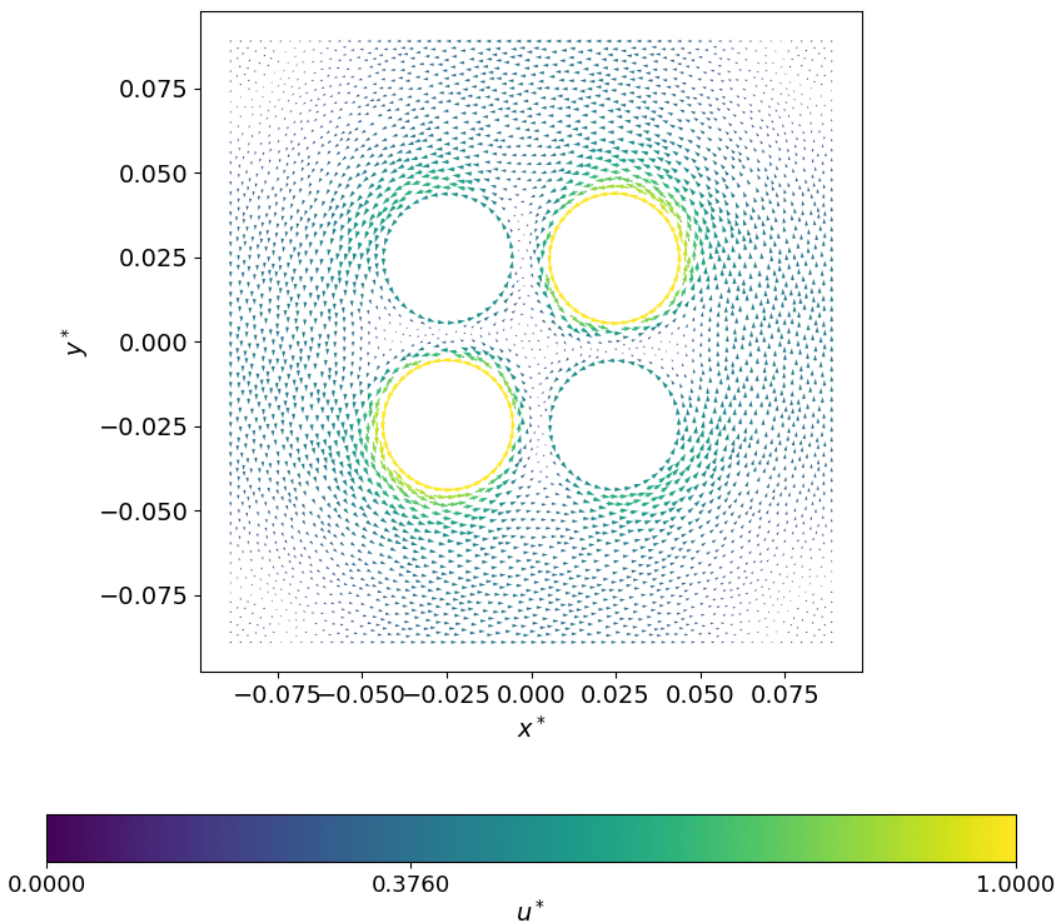

iv) $\lambda=-0.5$

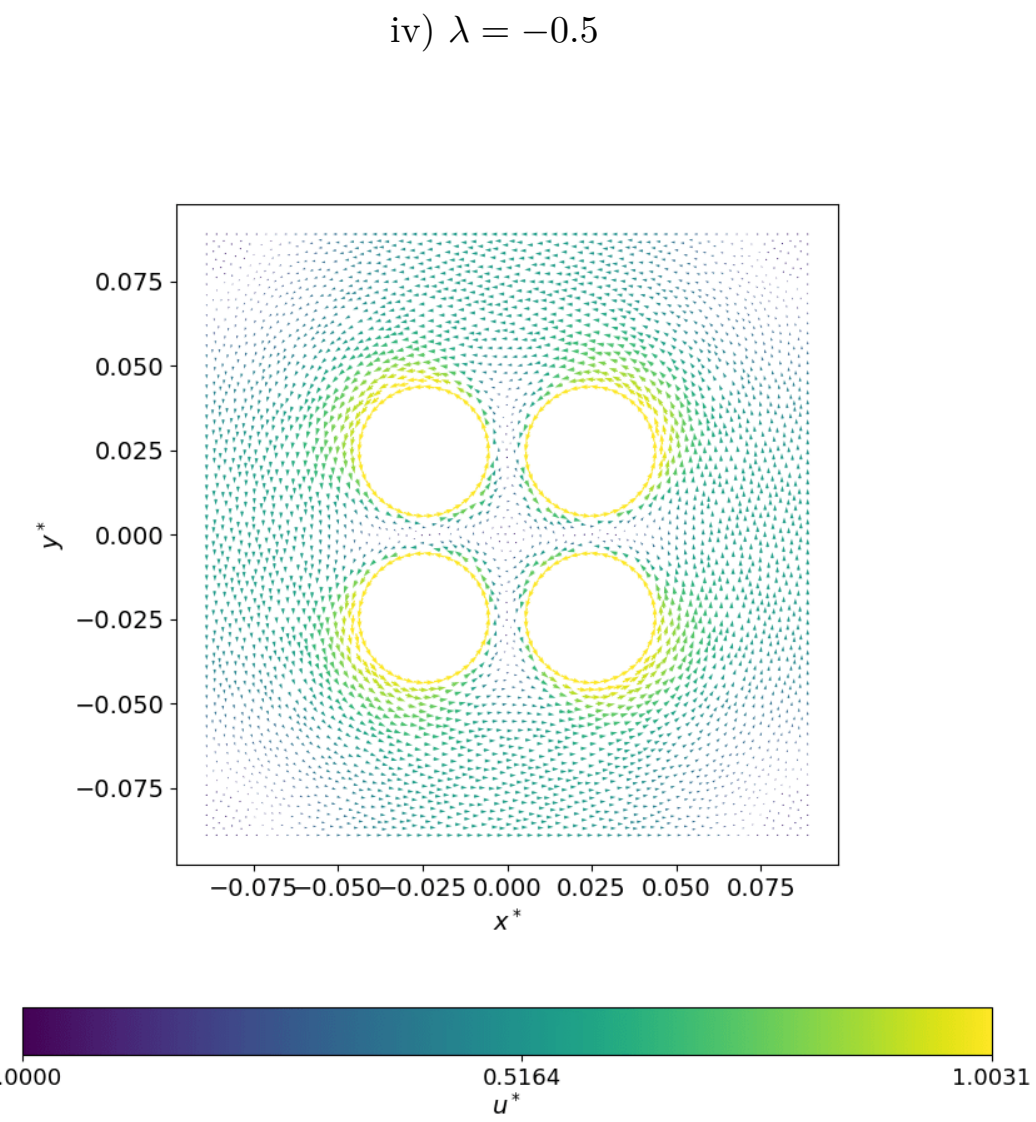

v) $\lambda=-1$

Figure 4.1: Non-dimensional velocity field in the classic four-roll mill with distinct flow configurations. 


\subsection{2}

\section{Streamlines}

Figure 4.2 presents the streamlines of each flow configuration. It is easy to assure that the streamlines schematics suggested by Lagnado [3], in the region between rollers on the four-roll mill, are quite accurate. The only discrepancy about them and the simulations employed in this work is visible in Figure 4.2 iii), in which $\lambda=0$, and streamlines are in fact still ellipses, but more flattened comparing to the figure $4.2 \mathrm{iv}$ ), where $\lambda=-0.5$. Nonetheless, the streamlines in the external region are also relevant to the describe the four-roll mill flow and are frequently left aside. In the majority of cases, since it is a steady flow, the external closed streamlines purely express a pathline around the cylinders, as can be seen in Figures $4.2 \mathrm{iii}$ ), $4.2 \mathrm{iv})$, and $4.2 \mathrm{v}$ ). On the other hand, zones of re-circulation appear in plot $4.2 \mathrm{i}$ ) near to each roller, but not around them. Interestingly, the flow configuration displayed in the figure 4.2 ii) shows both of these effects.

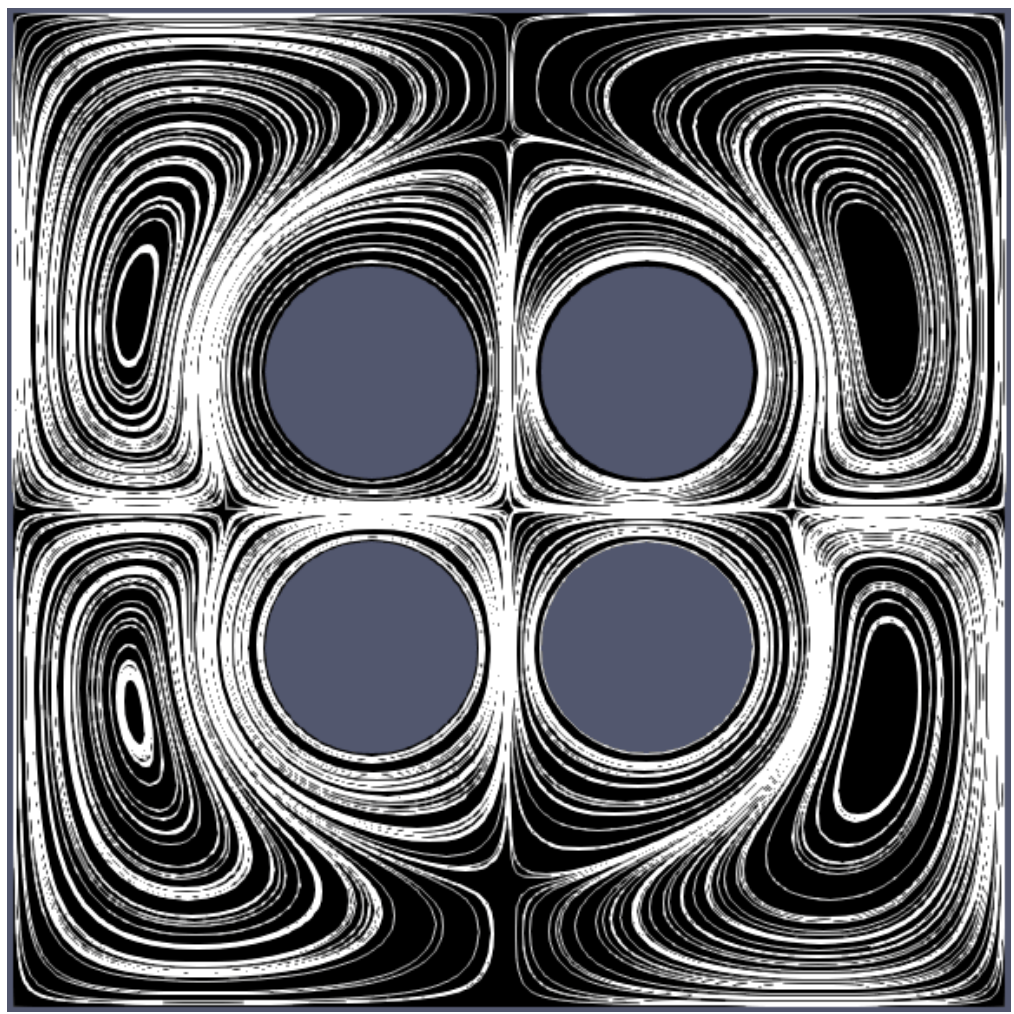

i) $\lambda=1$ 


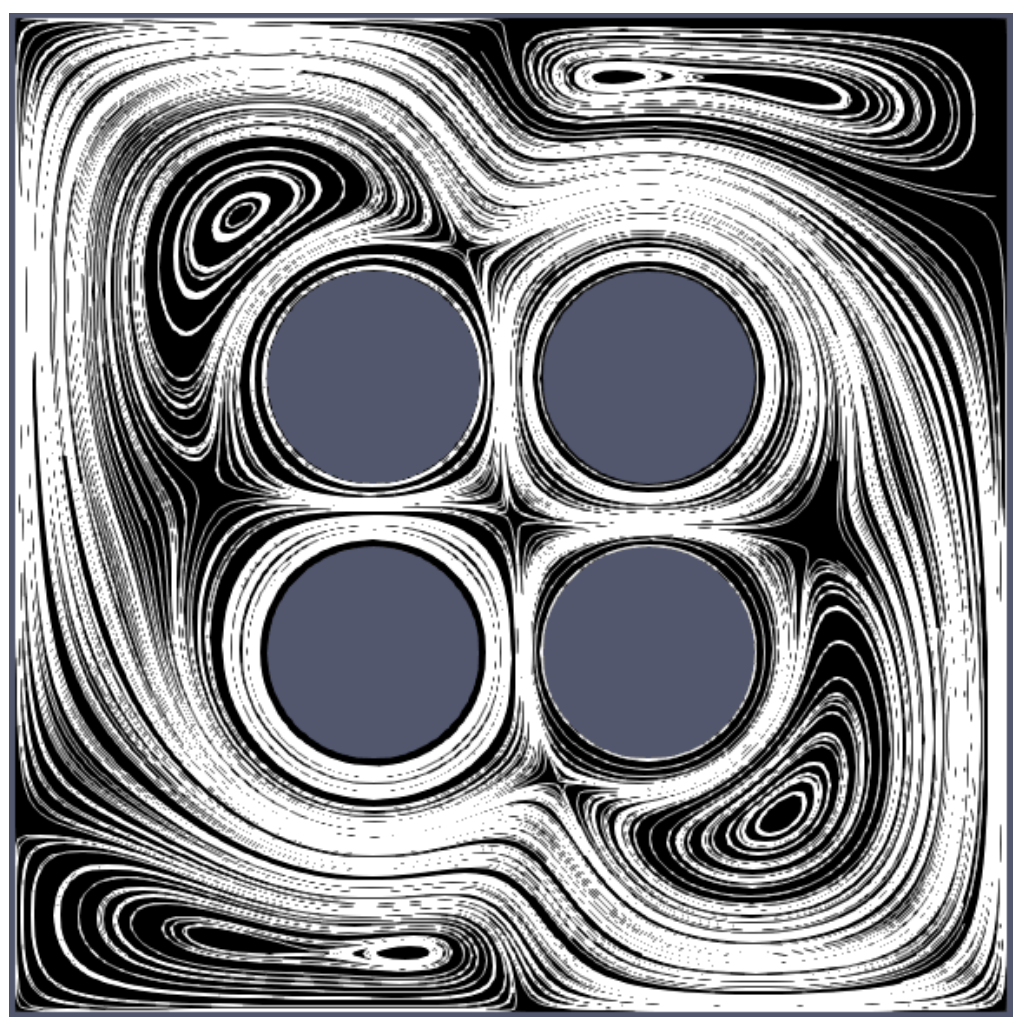

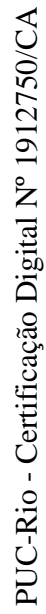

ii) $\lambda=0.5$

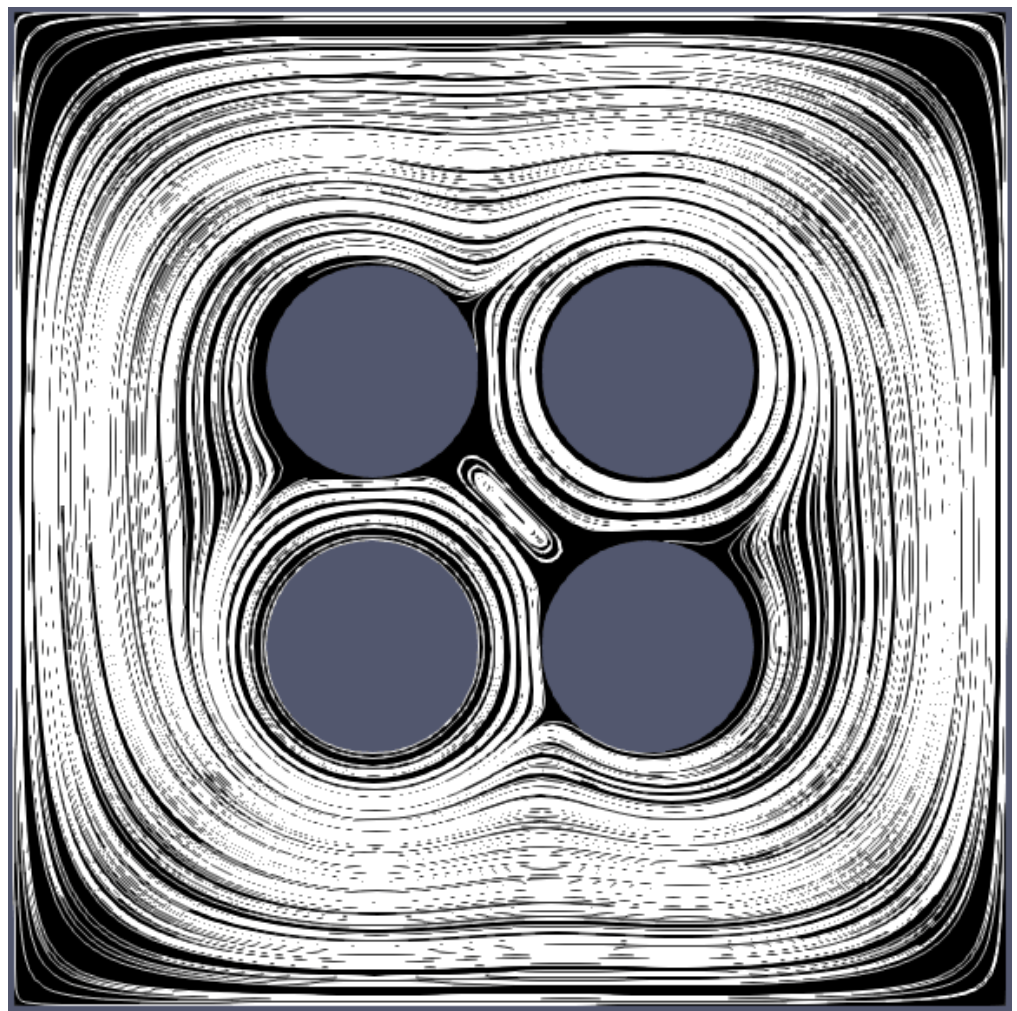

iii) $\lambda=0$ 


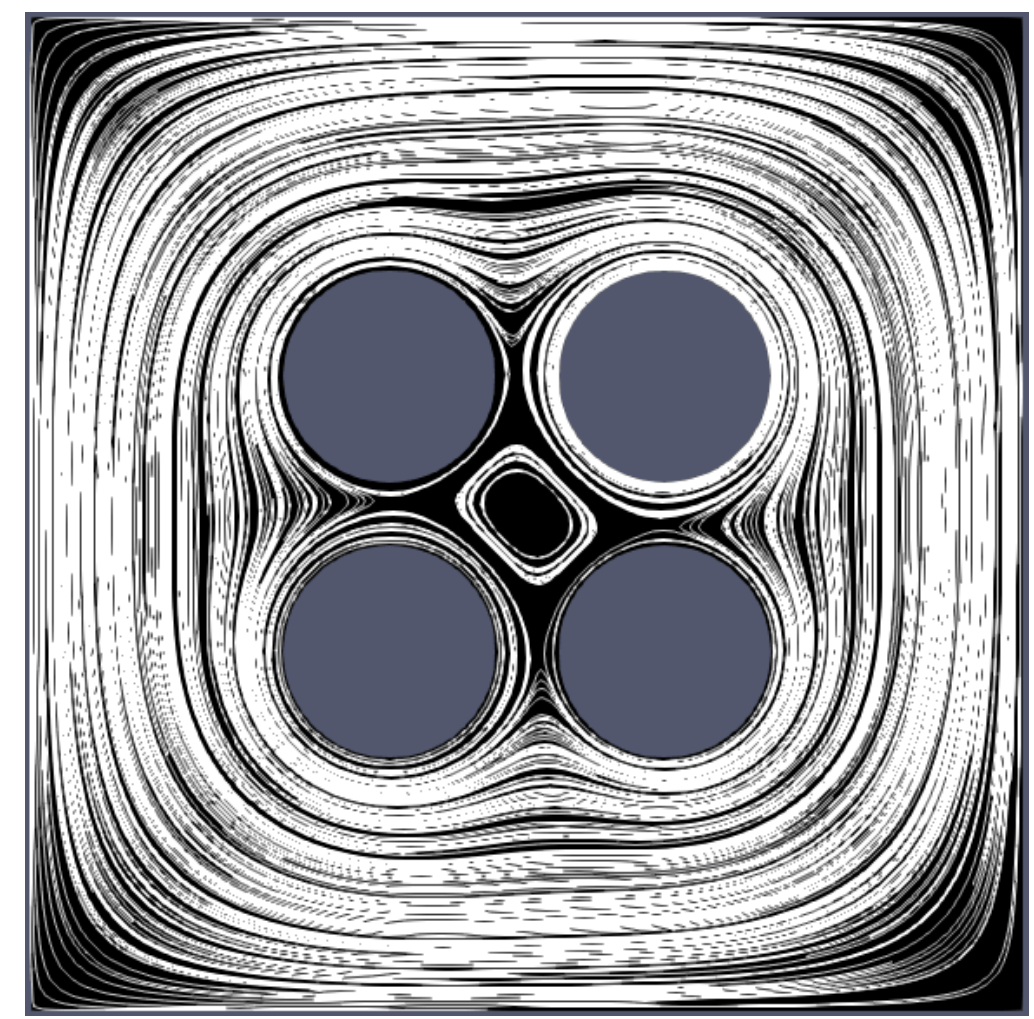

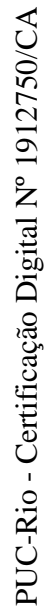

iv) $\lambda=-0.5$

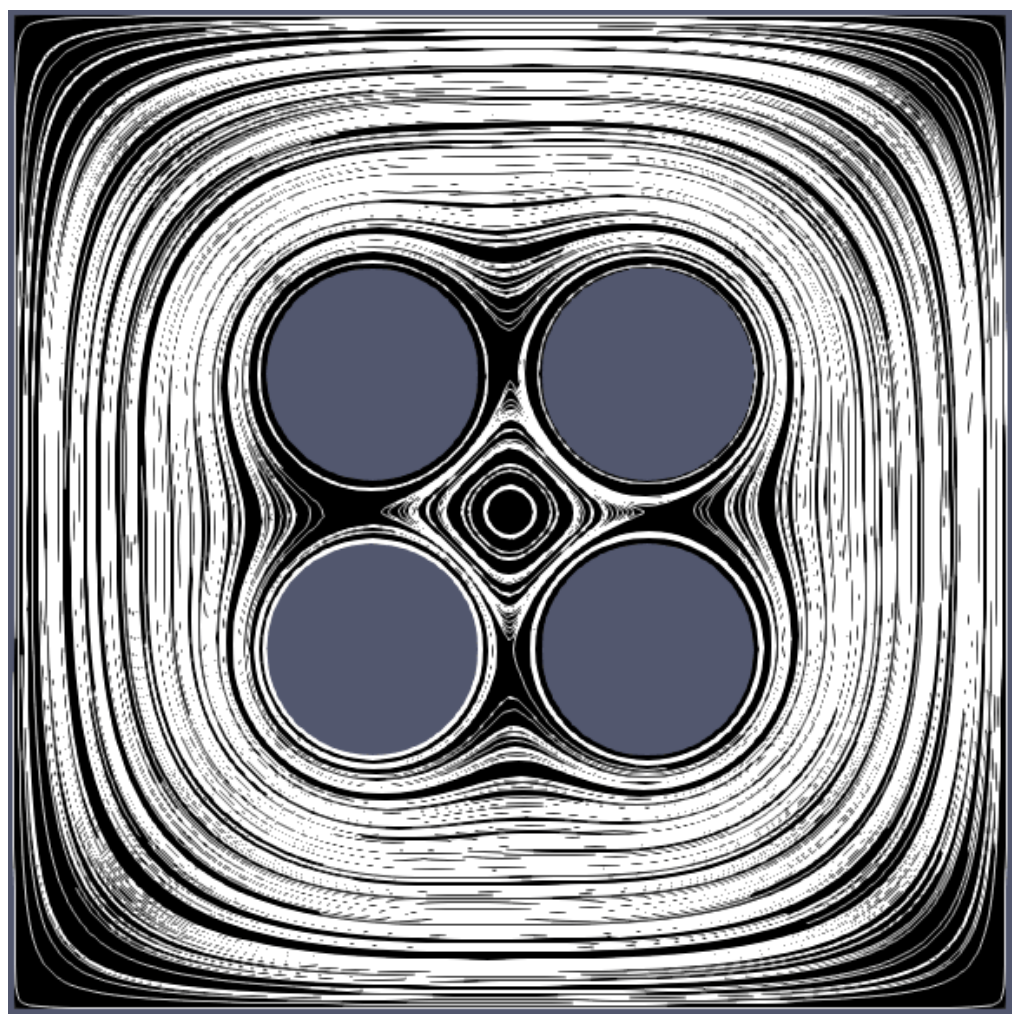

v) $\lambda=-1$

Figure 4.2: Streamlines in the classic four-roll mill with distinct flow configurations. 


\subsection{3}

\section{The normalized persistence-of-straining parameter field}

Previous works make use of the flow classification described in Table 2.1. However, they do not elucidate the details related to the local classification. Most of the times, different regions of the four-roll mill have completely distinct flow types. Even the exact same region usually display simultaneous behaviors, for instance extension and shear at the same time. Therefore, a global classification is too much vague to understand and describe the local kinematic behaviors in the four-roll mill.

Figure 4.3 shows the normalized persistence-of-straining parameter field for each flow configuration. The arrangement of angular velocities provided in Figure $4.3 \mathrm{i}$ ) is defined in the literature as pure extensional flow, i.e., associated with $\lambda=1$. As it can be seen, extensional flow is in fact dominant near the center of the four-roll mill. Between the rollers, where the velocities are higher, a clear rigid body movement is obtained. Since the rollers have the same tangent velocity, this context is analogous to a flow between walls moving with identical velocities. Near the cylinders borders, the fluid simultaneously deal with extension and shear flow. Although it is evident that the four-roll mill favors elongation in this specific configuration, the local classification is able to identify some regions that simple shear flow is in fact dominant.

Figure 4.3 iii) presents the classification known as simple shear flow. As a matter of fact, simple shear flow is experienced at the borders from the static cylinders. On the other side, at the rotating rollers, a combination of shear and extensional flow is identified. In the regions near where the boundaries intersect, the tangent velocity slowly decreases until becoming zero at the wall. This particularity results in regions of extension near the border due to the local stagnation flow.

The rotational flow described by the literature is exhibited in Figure 4.3 v). In the internal region, the result is almost the opposite from case i). Near the origin, we have a rigid body movement related to a internal rotational flow. Between the rollers, a mix of shear and extensional flow is established. In the external region, another rotational movement is recognized with velocities close to the average from the velocity field, resulting in regions of shear flow and rigid body motion combined and other regions with exclusive rigid body motion.

Notice that the flow configurations presented in Figures $4.3 \mathrm{ii}$ ) and 4.3 iv) are sightly variations of $4.3 \mathrm{i}$ ) and $4.3 \mathrm{v}$ ) respectively. Interestingly, the presence of shear flow in these variations $(\lambda=0.5$ and $\lambda=-0.5)$ increases comparing to the case defined as simple shear flow $(\lambda=0)$ in the literature. The 
reason is related to the intensification of the occurrence of angular strain due to differences between the velocity of rollers. This shows one more inconsistency of the global criteria for flow classification currently used.

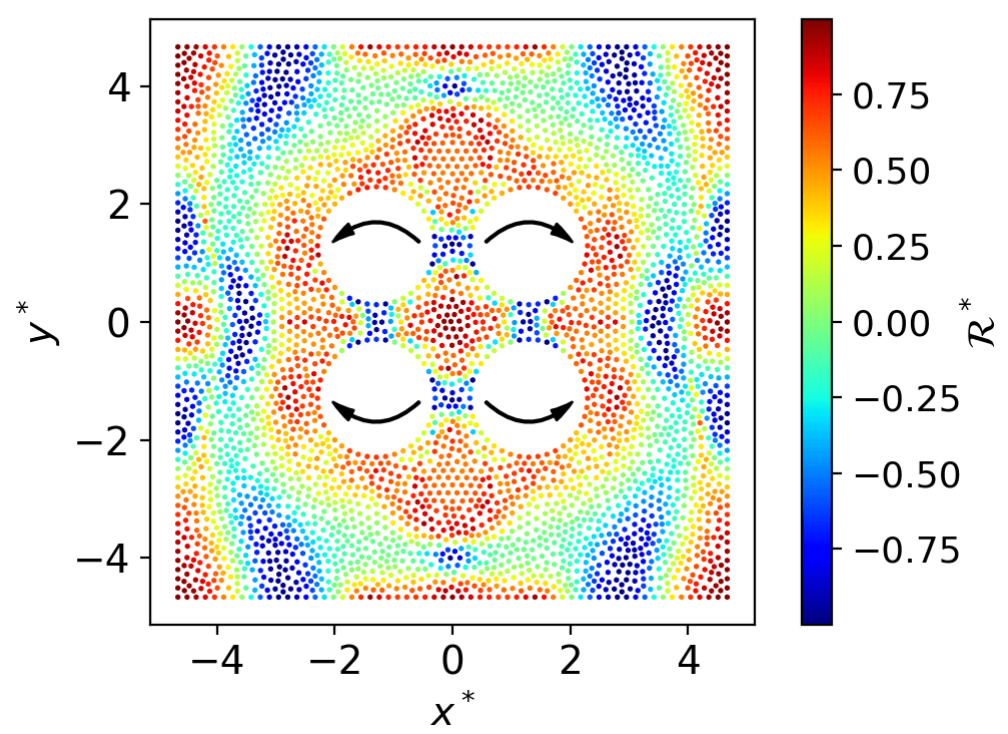

i) $\lambda=1$

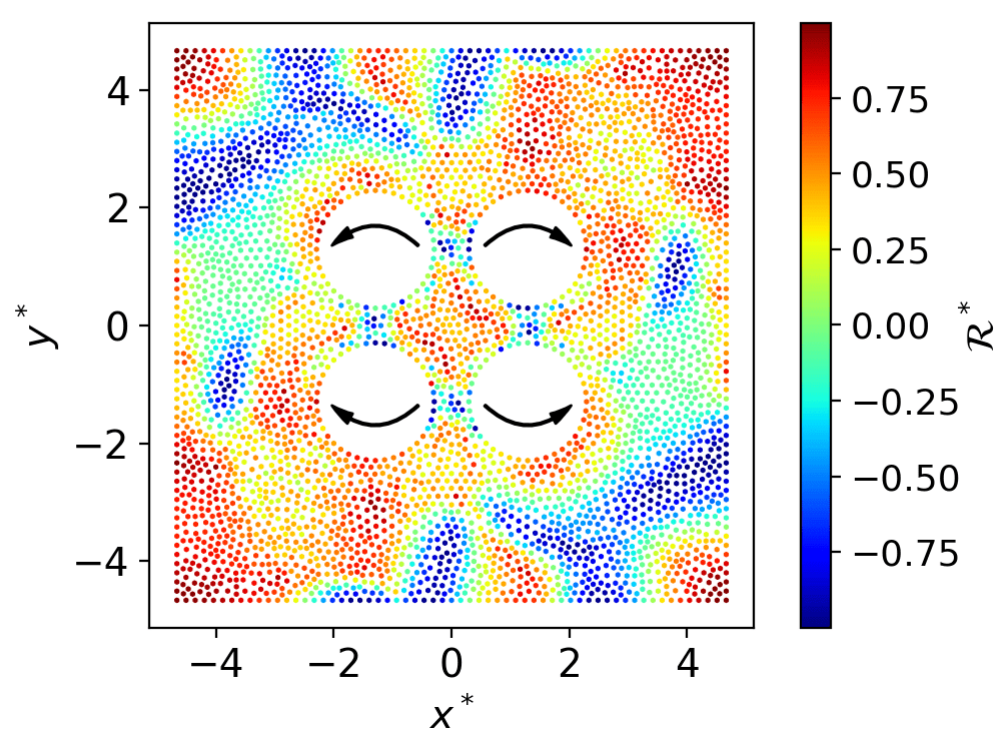

ii) $\lambda=0.5$ 


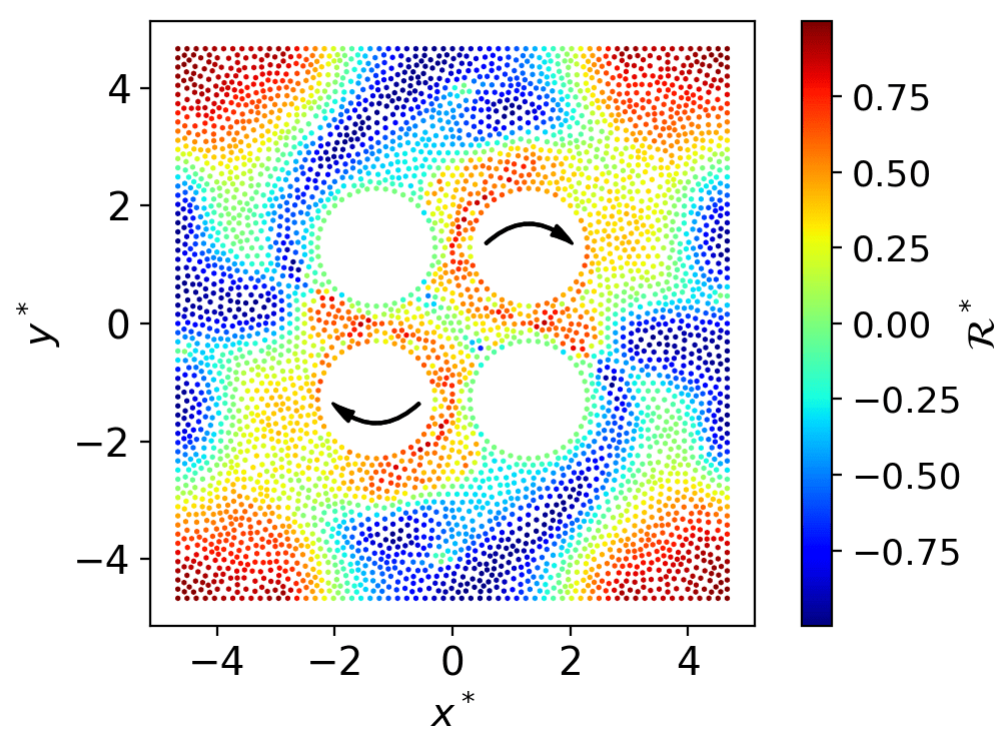

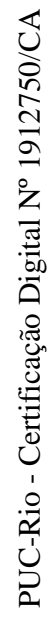

iii) $\lambda=0$

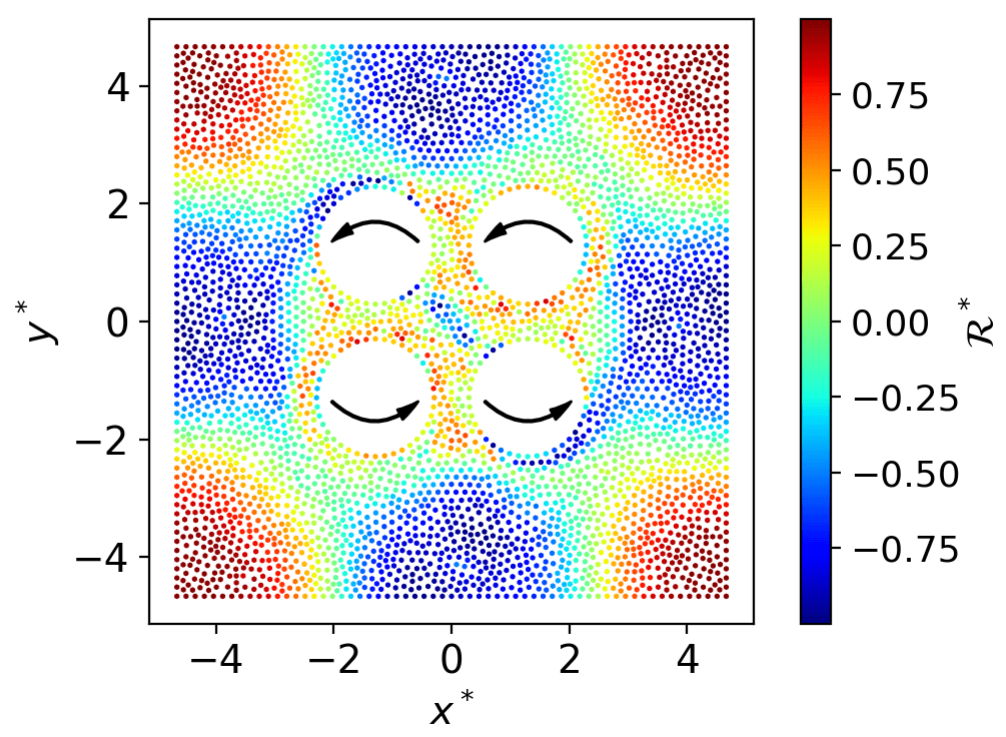

iv) $\lambda=-0.5$ 


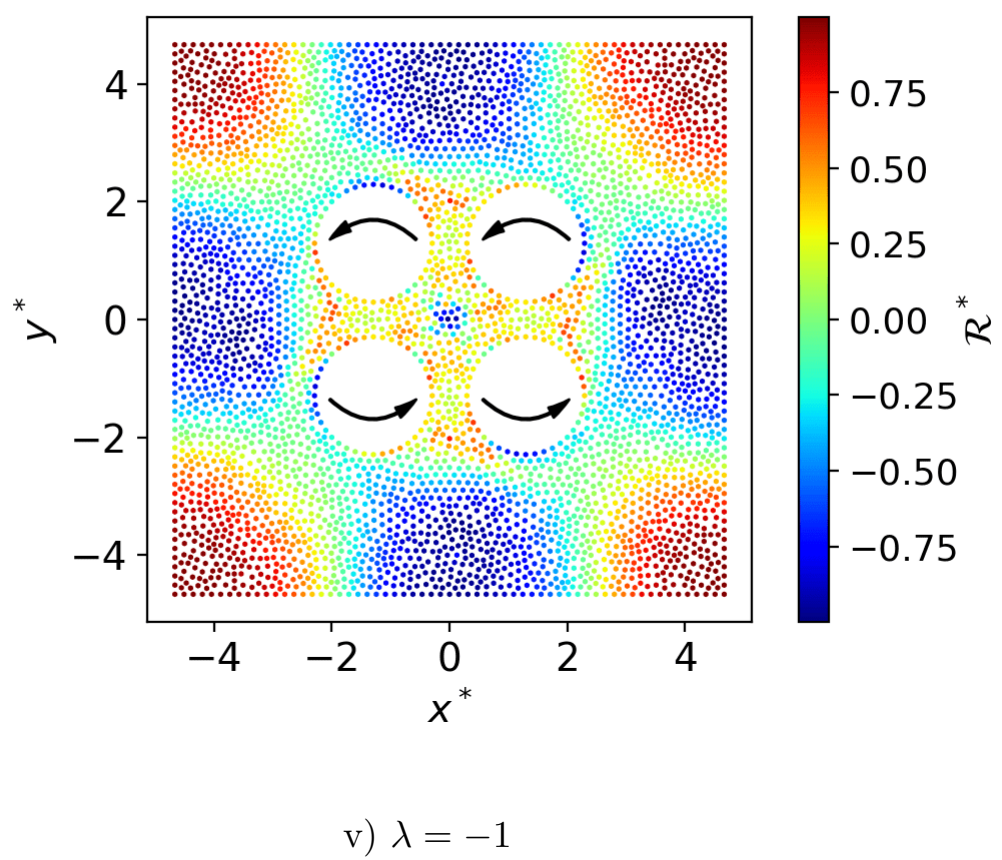

Figure 4.3: $\mathcal{R}^{*}$ field in the classic four-roll mill with distinct flow configurations.

\subsection{4 \\ The modified normalized persistence-of-straining parameter field}

The modified normalized persistence-of-straining parameter field interprets the flow classification of the dispersed phase. There are different kinematic behaviors, at the same position, depending on which phase of emulsion is currently at this region. The results related to this specific field are an attempt to provide a flow classification if every single mesh node was filled with a microelement of the dispersed phase. The coefficients $G, \alpha$, and $F$ describe the microstrucutre of the microelement. For simplicity, $F=0$ is assumed. Otherwise, the modified normalized persistence-of-straining parameter field would change at each time step. Thus, the aim is to analyze the influence of the parameters $G$ and $\alpha$ on the microelement flow classification scheme.

For each flow configuration, distinct combinations of $G$ and $\alpha$ were simulated to obtain different modified normalized persistence-of-straining parameter fields. Figures 4.4, 4.5, and 4.6 show these fields for $\lambda=1, \lambda=0$, and $\lambda=-1$ respectively. As already mentioned, the particular case in which $G=1$, $\alpha=0$, and $F=0$ represents the only case where the dispersed phase behaves exactly as the continuous phase, i.e., $\mathcal{R}^{*}{ }_{\text {mod }}=\mathcal{R}^{*}$. Consequently, the cases presented in Figures 4.4 vii), 4.5 vii), and 4.6 vii) match exactly respectively with $4.3 \mathrm{i}$ ), 4.3 iii), 4.3 and v). 
Notice that for each flow configuration, the increase of the parameter $\alpha$ or $G$ results in the spread and rise of regions that represent pure extension or a mix of shear and extensional flow, i.e. $\mathcal{R}^{*}{ }_{\text {mod }} \in(0,1]$. This trend of extensional flow at first does not specify an elongation or compression behavior. On the other hand, the decrease of $G$ endorse the appearance of regions of rigid body motion and sometimes it is mixed with shear flow, i.e. $\mathcal{R}^{*}{ }_{\text {mod }} \in[-1,0)$. It is essential to emphasize that the transformation of the modified normalized persistence-of-straining parameter field with the variation of $\alpha$ and $G$ is totally gradual. For instance, the comparison of figures 4.4 vii), 4.4 viii), and 4.4 ix) illustrates the increase of $\alpha$ with $G=1$ fixed. As $\alpha$ is increased, the region that is initially classified as rigid body change to a classification of simple shear flow, while regions originally of shear flow become a mix of shear and extensional flow. Analogously, the region that initially represents a mix of shear and extensional flow shifts to a classification of pure extensional flow.

Other interesting cases are the ones with the minimum $G$ and maximum $\alpha$. In theses cases, i.e. plots $4.4 \mathrm{xv}$ ), $4.5 \mathrm{xv}$ ), and $4.6 \mathrm{xv}$ ), it can be seen that the extreme behaviors are both present. Near the rollers, the behavior of rigid body motion is dominant while near the borders, extensional flow is dominant instead. 


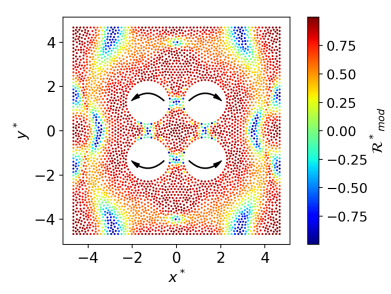

i) $G=4 ; \alpha=0$

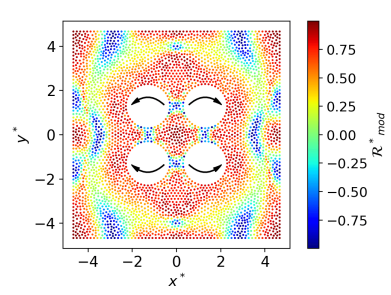

iv) $G=2 ; \alpha=0$

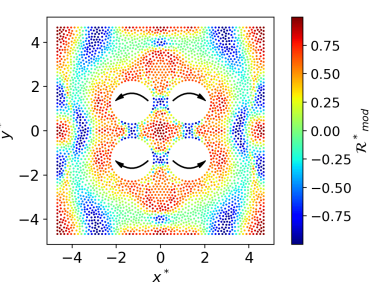

vii) $G=1 ; \alpha=0$

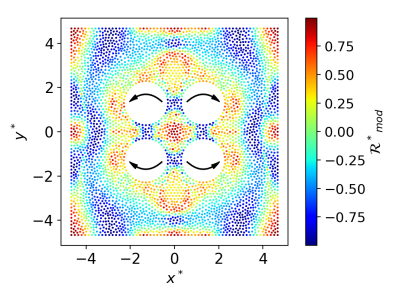

x) $G=0.5 ; \alpha=0$

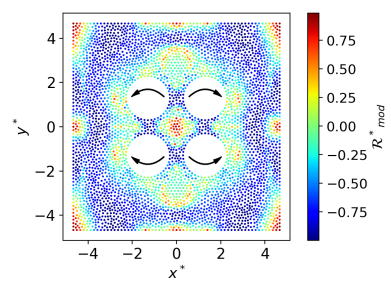

xiii) $G=0.2 ; \alpha=0$

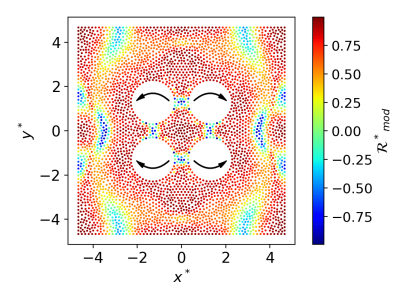

ii) $G=4 ; \alpha=0.2$

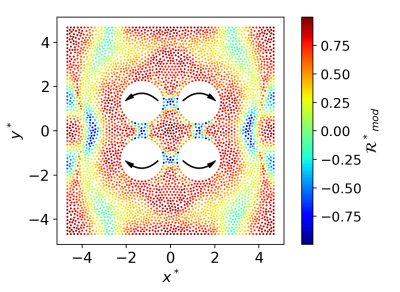

v) $G=2 ; \alpha=0.2$

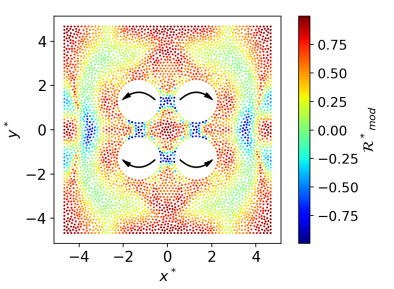

viii) $G=1 ; \alpha=0.2$

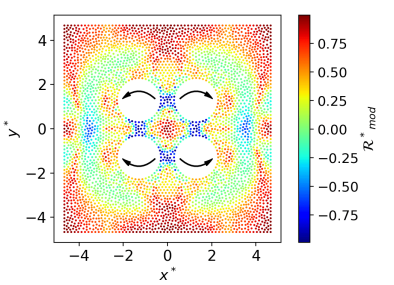

xi) $G=0.5 ; \alpha=0.2$

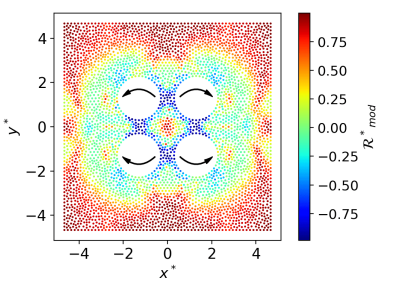

xiv) $G=0.2 ; \alpha=0.2$

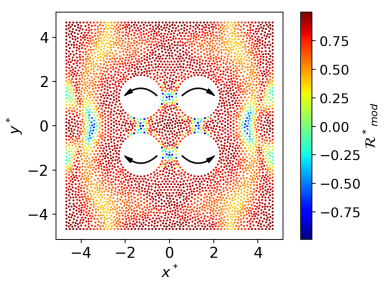

iii) $G=4 ; \alpha=0.5$

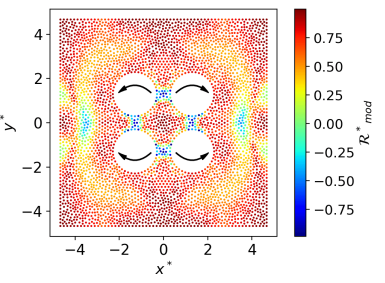

vi) $G=2 ; \alpha=0.5$

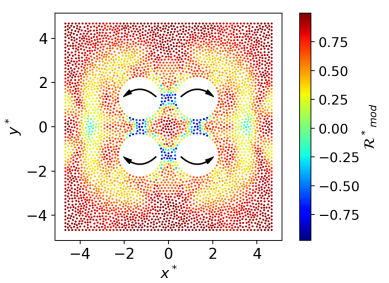

ix) $G=1 ; \alpha=0.5$

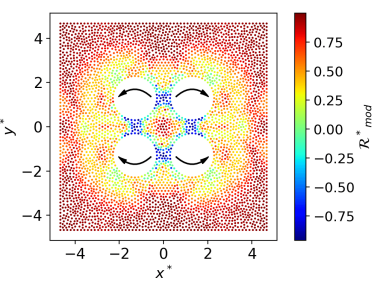

xii) $G=0.5 ; \alpha=0.5$

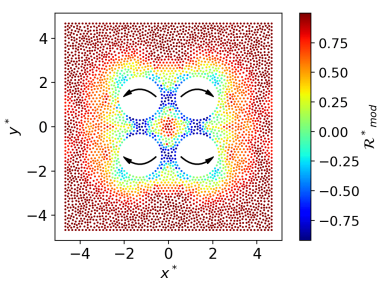

xv) $G=0.2 ; \alpha=0.5$

Figure 4.4: $\mathcal{R}^{*}$ mod field in the classic four mill with $\lambda=1$ and distinct microelements. 


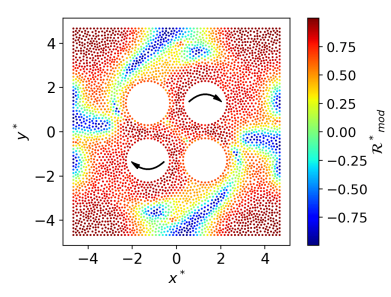

i) $G=5 ; \alpha=0$

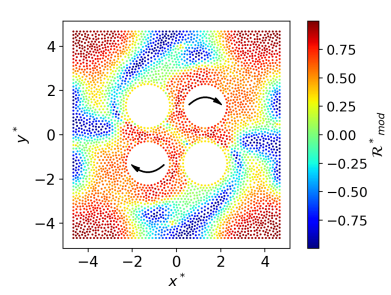

iv) $G=2 ; \alpha=0$

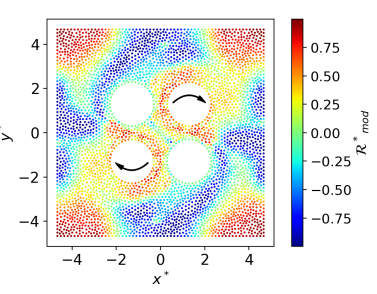

vii) $G=1 ; \alpha=0$

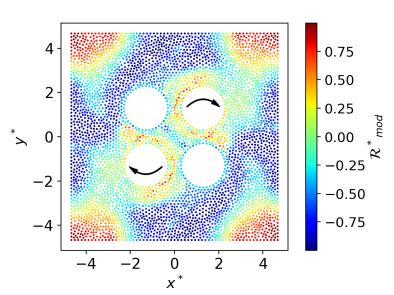

x) $G=0.5 ; \alpha=0$

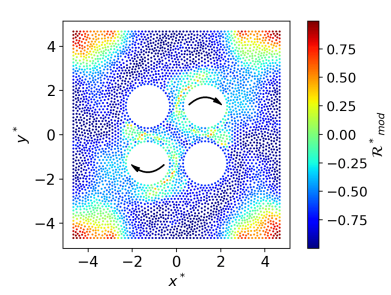

xiii) $G=0.2 ; \alpha=0$

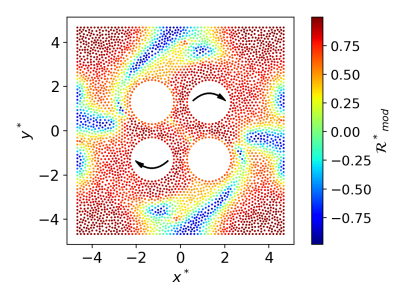

ii) $G=5 ; \alpha=0.1$

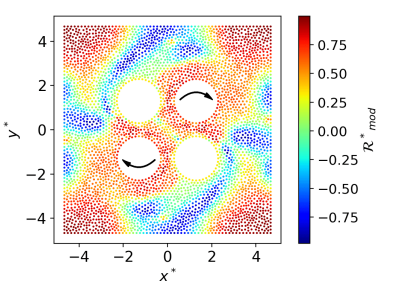

v) $G=1 ; \alpha=0.1$

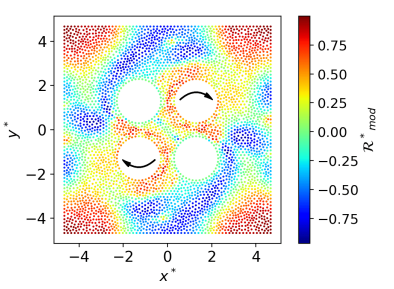

viii) $G=1 ; \alpha=0.1$

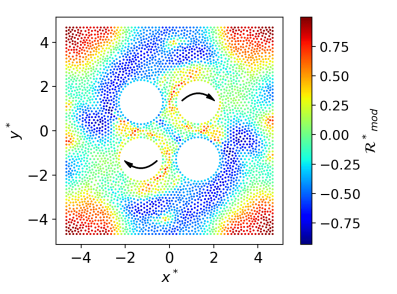

xi) $G=0.5 ; \alpha=0.1$

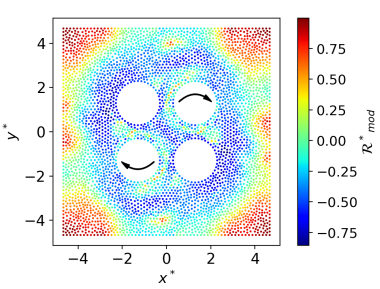

xiv) $G=0.2 ; \alpha=0.1$

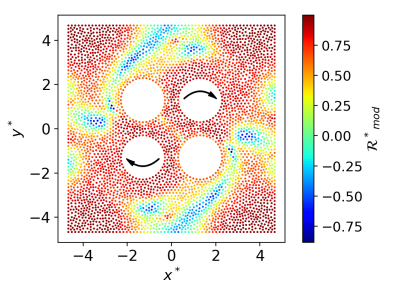

iii) $G=5 ; \alpha=0.4$

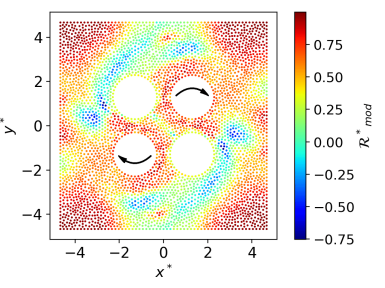

vi) $G=1 ; \alpha=0.4$

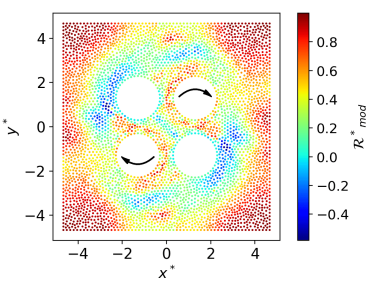

ix) $G=1 ; \alpha=0.4$

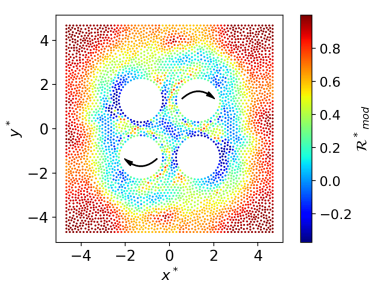

xii) $G=0.5 ; \alpha=0.4$

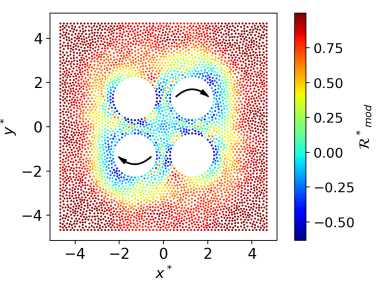

xv) $G=0.2 ; \alpha=0.4$

Figure 4.5: $\mathcal{R}^{*}{ }_{\text {mod }}$ field in the classic four mill with $\lambda=0$ and distinct microelements. 


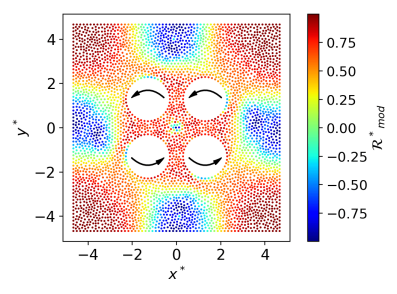

i) $G=4 ; \alpha=0$

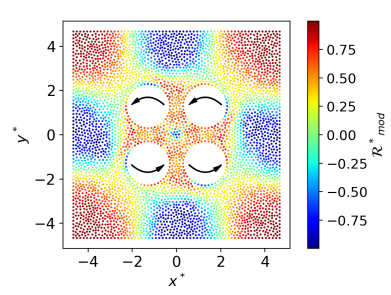

iv) $G=2 ; \alpha=0$

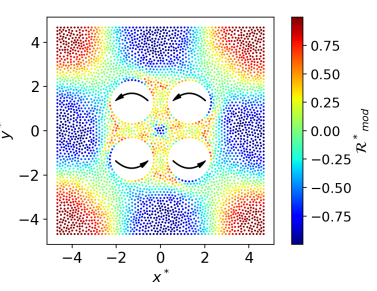

vii) $G=1 ; \alpha=0$

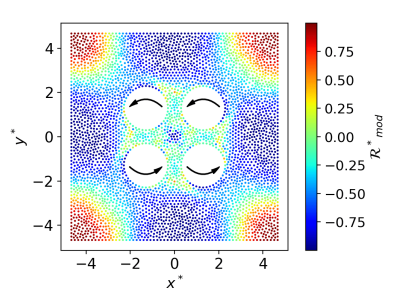

x) $G=0.4 ; \alpha=0$

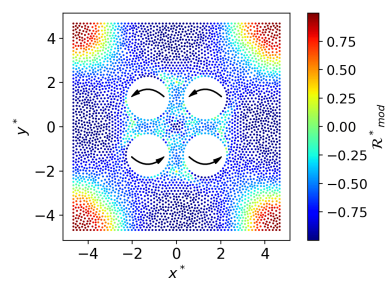

xiii) $G=0.2 ; \alpha=0$

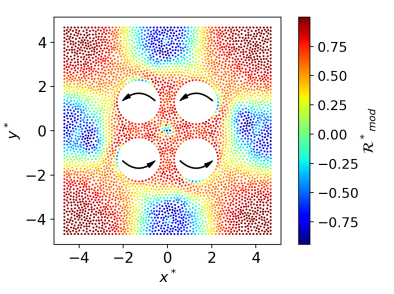

ii) $G=4 ; \alpha=0.2$

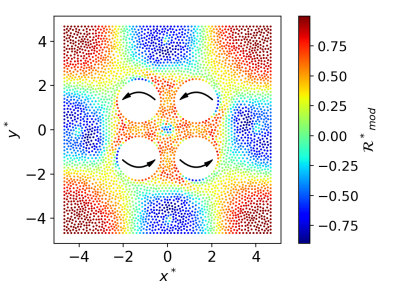

v) $G=2 ; \alpha=0.2$

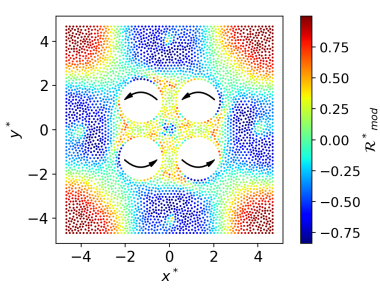

viii) $G=1 ; \alpha=0.2$

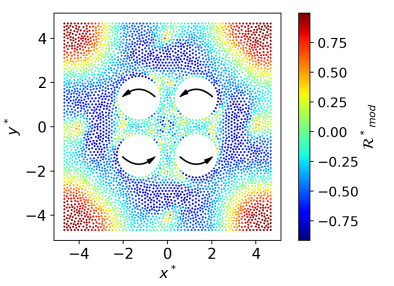

xi) $G=0.4 ; \alpha=0.2$

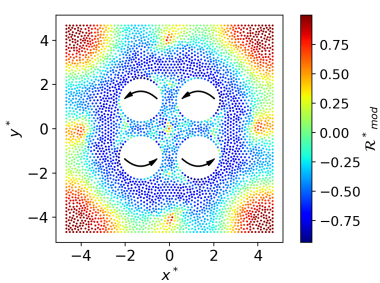

xiv) $G=0.2 ; \alpha=0.2$

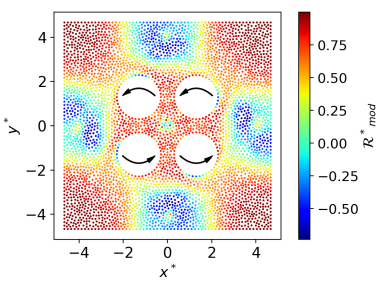

iii) $G=4 ; \alpha=0.5$

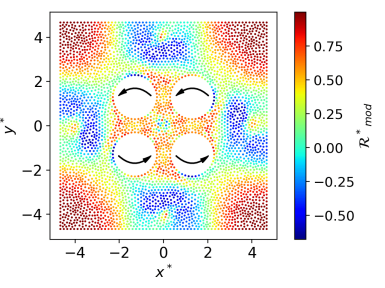

vi) $G=2 ; \alpha=0.5$

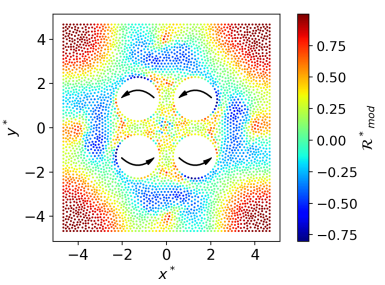

ix) $G=1 ; \alpha=0.5$

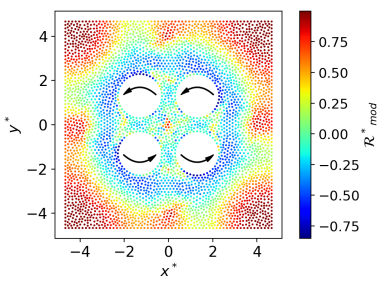

xii) $G=0.4 ; \alpha=0.5$

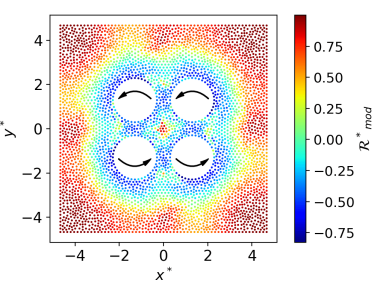

xv) $G=0.2 ; \alpha=0.5$

Figure 4.6: $\mathcal{R}^{*}{ }_{\bmod }$ field in the classic four mill with $\lambda=-1$ and distinct microelements. 


\section{2}

\section{Expanded four-roll mill}

The main point of the expanded four-roll mill is to get closer to the motivation of using the four-roll mill as a system to contribute to phase separation. Analogously, distinct flow configurations are investigated on the nondimensional velocity fields, streamlines, normalized persistence-of-straining parameter field, and modified normalized persistence-of-straining parameter field from the expanded four-roll mill. Non-null entrance Reynolds numbers are examined. Therefore, it allows the inspection of the deformation of several microelements with distinct behaviors that are initially placed at the inlet border.

\subsection{1}

\section{Velocity fields}

Figures 4.7, 4.8, and 4.9 represent respectively the velocities fields on the expanded four-roll mill of $\lambda=1, \lambda=0$, and $\lambda=-1$ with different sets of entrance Reynolds numbers. Notice that Figures 4.7 i), 4.8 i), and 4.9 i) represent the cases of different flow configurations with $R e_{e n t}=0$. Notice that these specific cases differ from the analogous cases related to plots $4.1 \mathrm{i}$ ), 4.1 iii), and $4.1 \mathrm{v}$ ) of the classic four-roll mill mainly because of the domain size. However, in the internal region, the results of velocity field are almost the same, both from the quantitative and qualitative points of view.

In each flow configuration, the effect of the entrance Reynolds is analyzed. As it can be seen more clearly in Figures $4.7 \mathrm{v}$ ), $4.8 \mathrm{v}$ ), and $4.9 \mathrm{v}$ ), which are cases with $R e_{e n t}=10$, as the entrance Reynolds number is increased the flow becomes more similar to the flow around a rotating cylinder. Despite the different flow configurations, these outputs are very much alike. Besides the internal region, the location which has the higher flow velocity is what mainly differs the results for each flow configuration. For instance, in Figure $4.9 \mathrm{v}$ ), the region below the roller has higher velocities than the region above the rollers. On the other hand, the opposite is clearly visible in Figure $4.8 \mathrm{v}$ ), while the plot $4.7 \mathrm{v}$ ) displays the exact same velocities above and below the cylinders. 

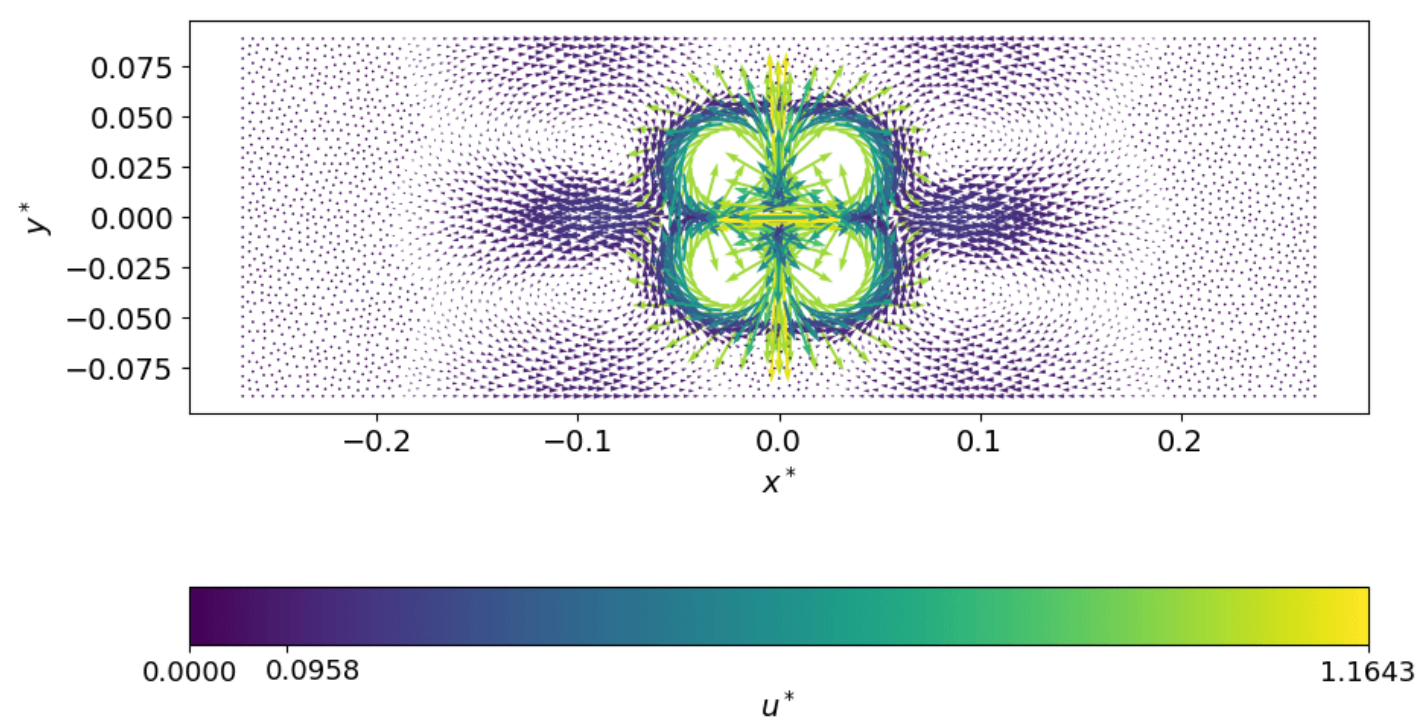

i) $R e_{\text {ent }}=0$

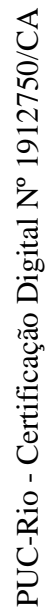

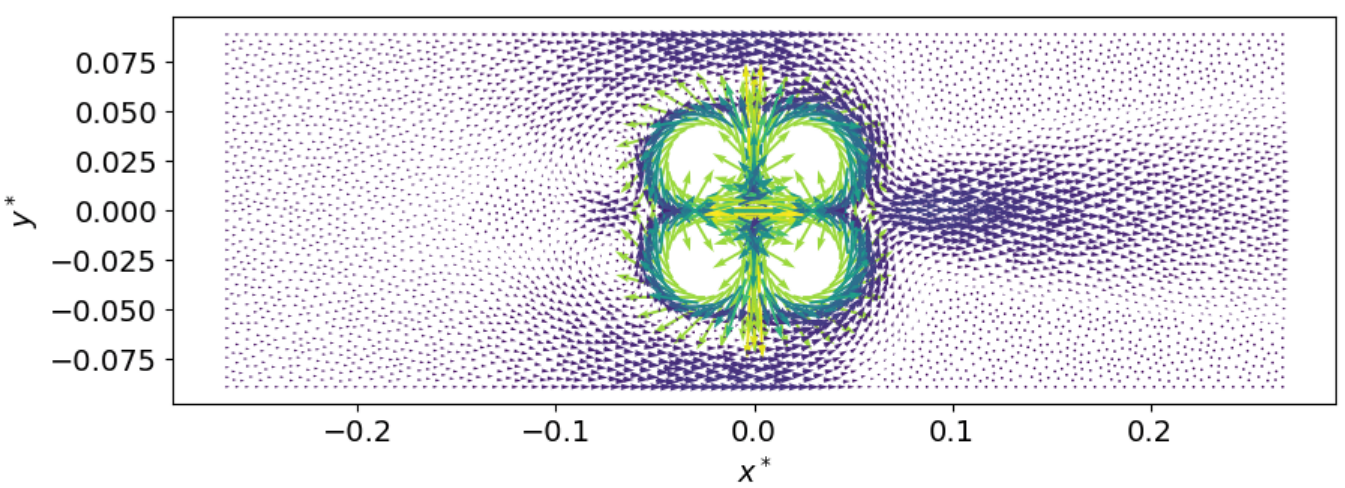

0.00010 .1118

$u^{*}$

1.1784

ii) $R e_{\text {ent }}=1$ 

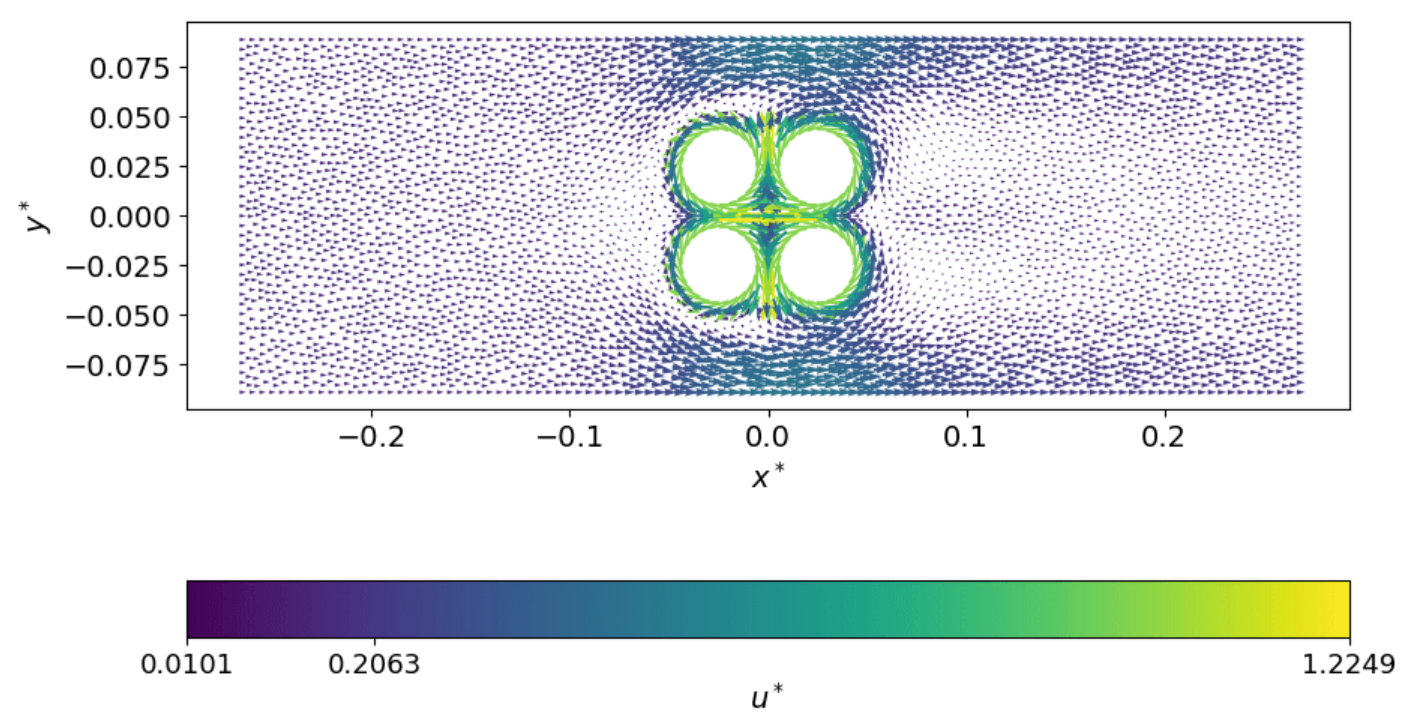

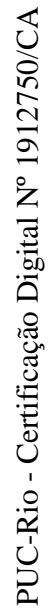

iii) $R e_{\text {ent }}=3$

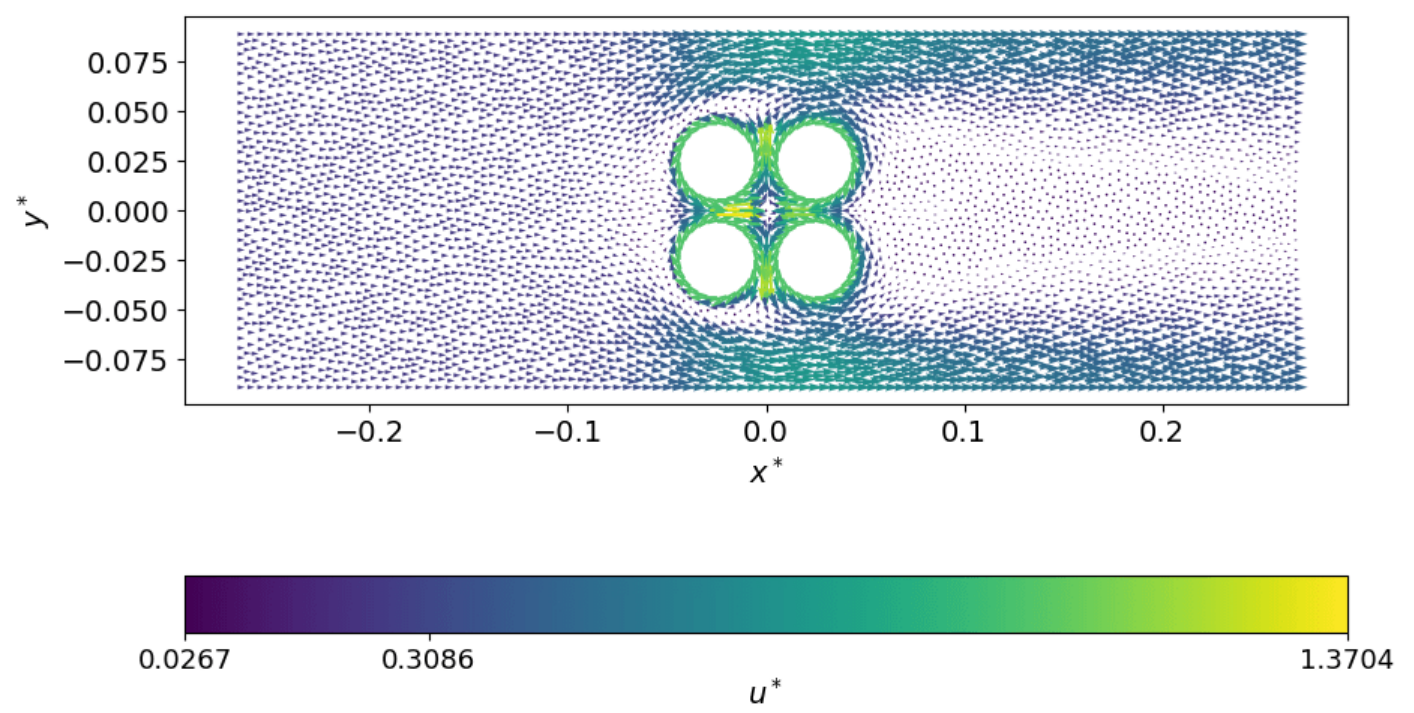

iv) $R e_{e n t}=5$ 

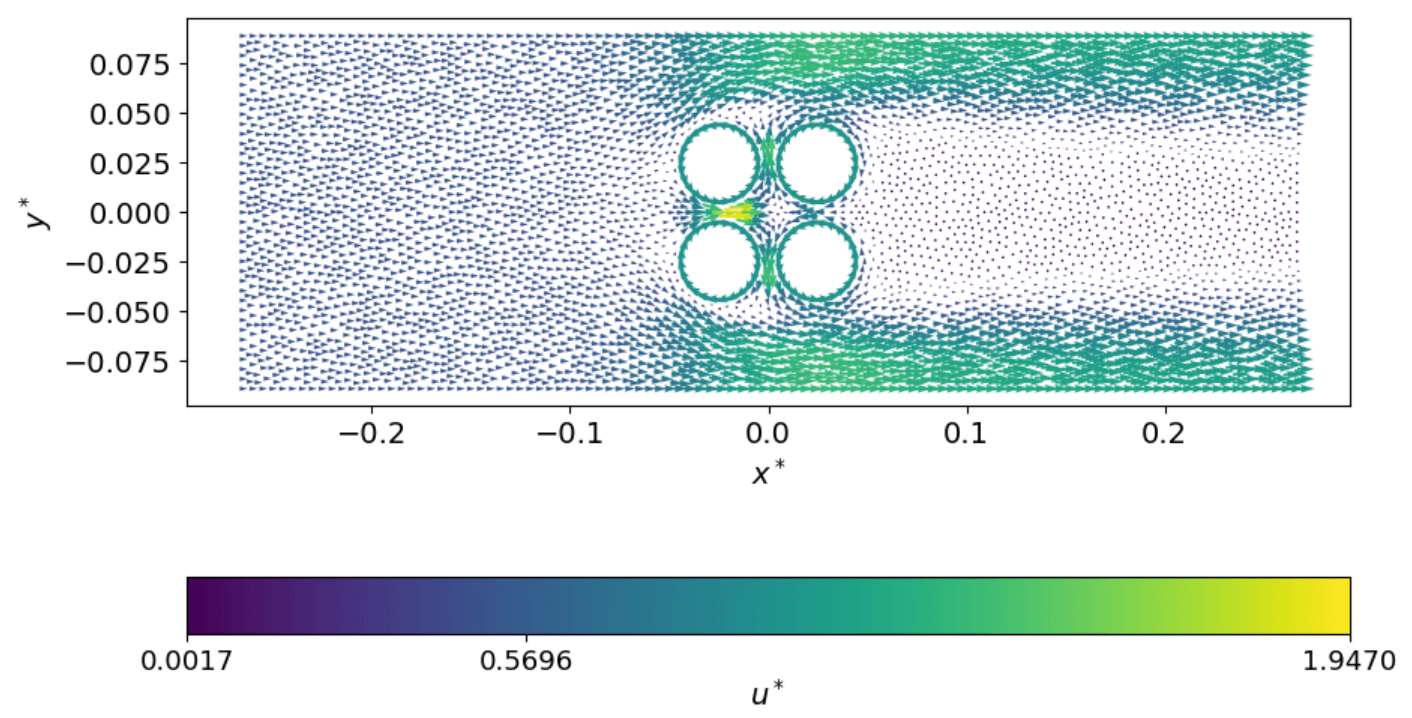

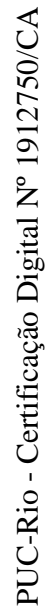

v) $R e_{\text {ent }}=10$

Figure 4.7: Velocity field in the expanded four-roll mill with $\lambda=1$ and distinct entrance Reynolds number.
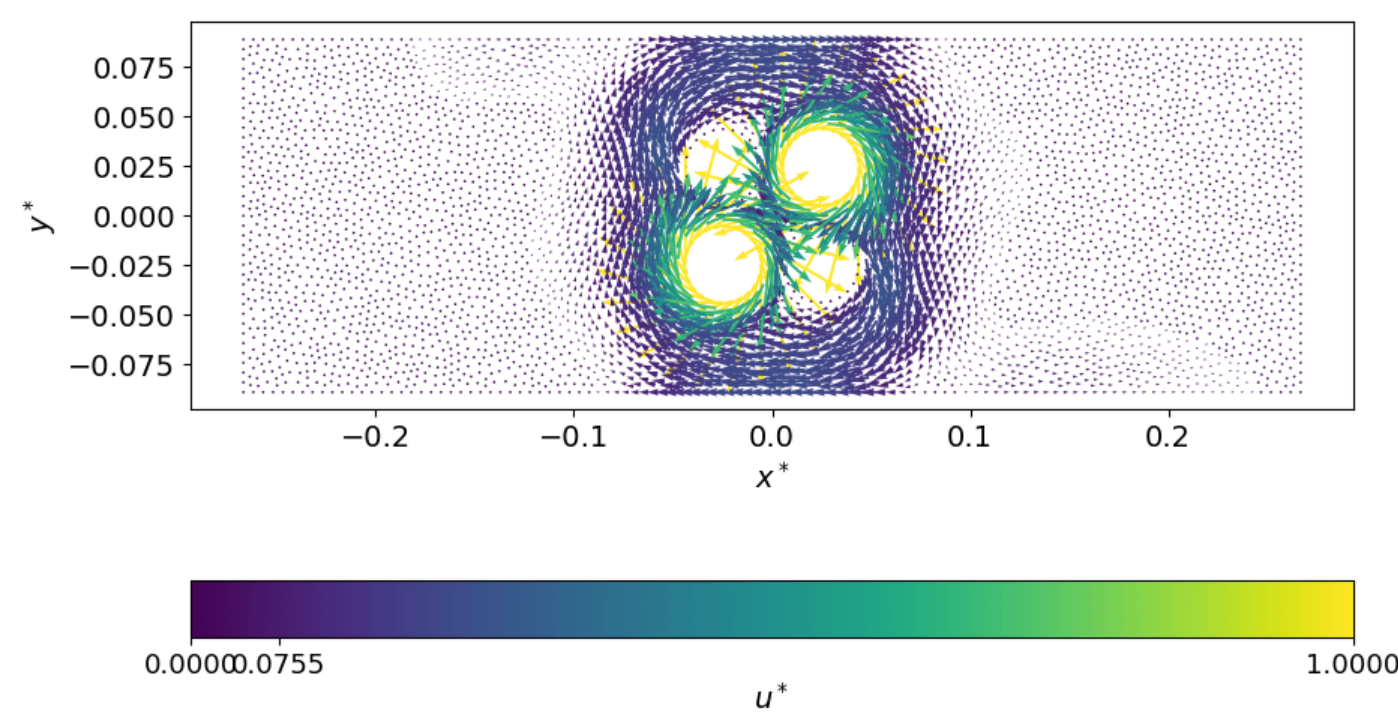

i) $R e_{\text {ent }}=0$ 

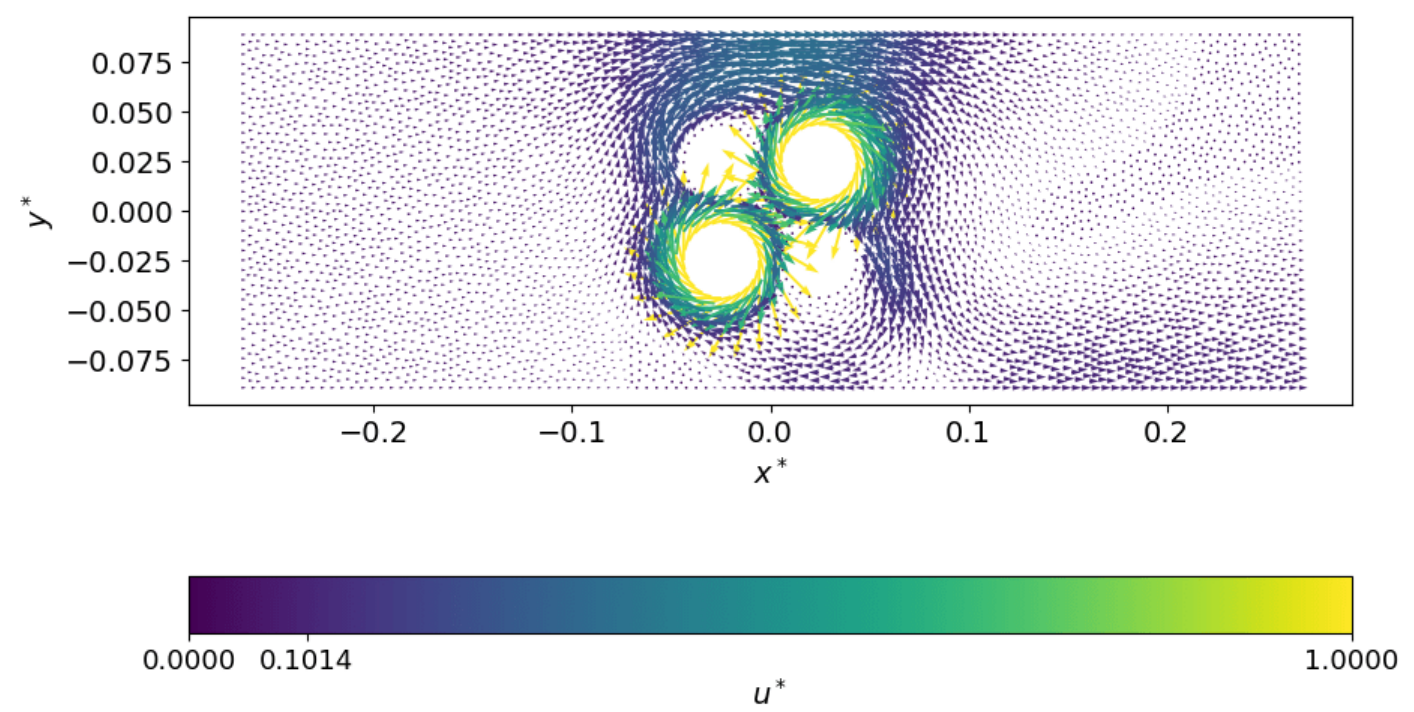

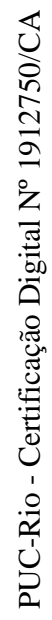

ii) $R e_{\text {ent }}=1$

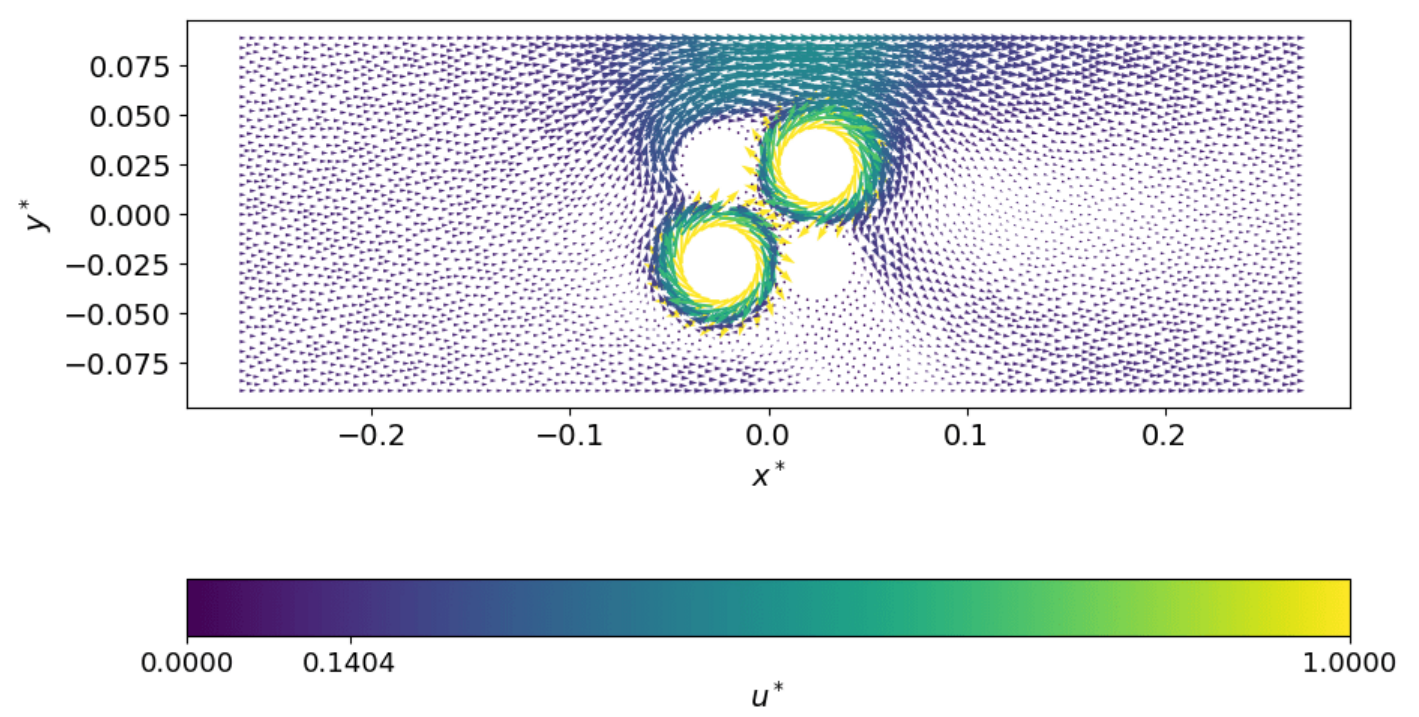

iii) $R e_{\text {ent }}=2$ 

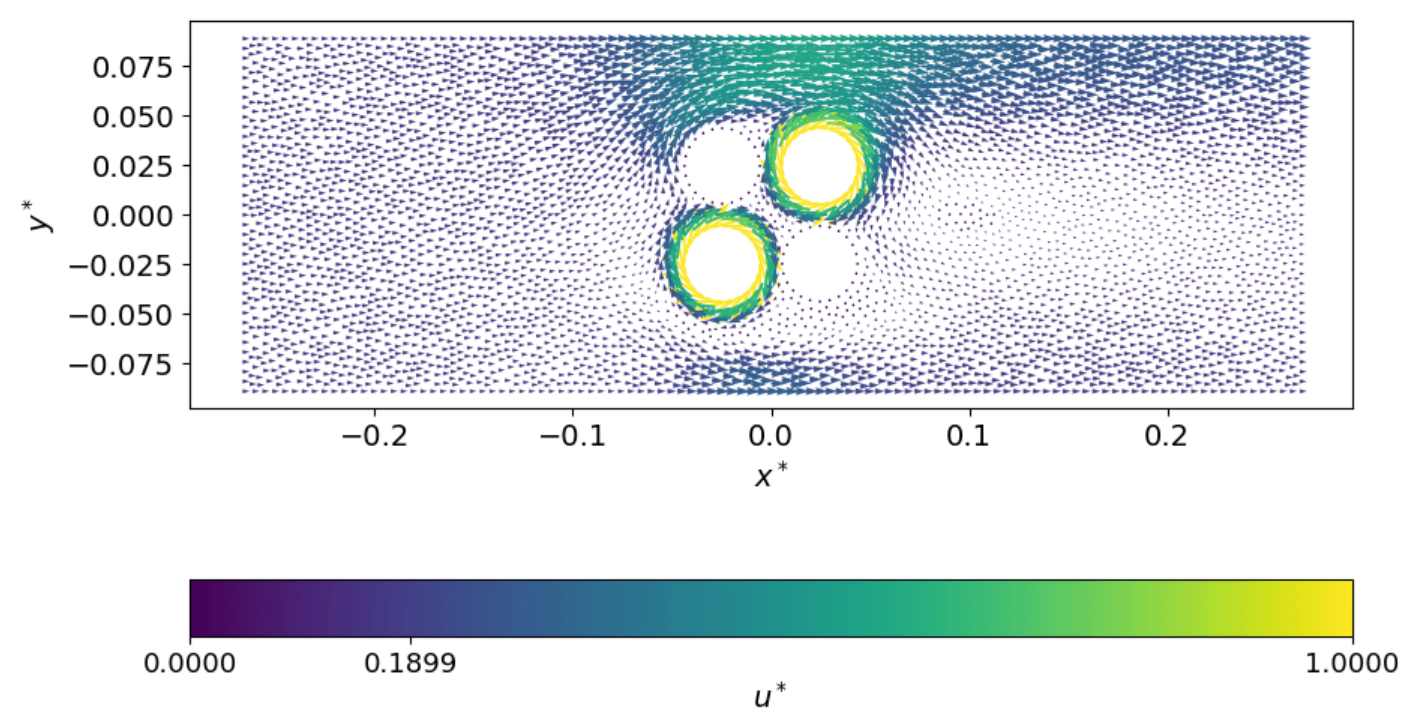

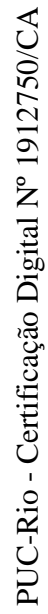

iv) $R e_{\text {ent }}=3$
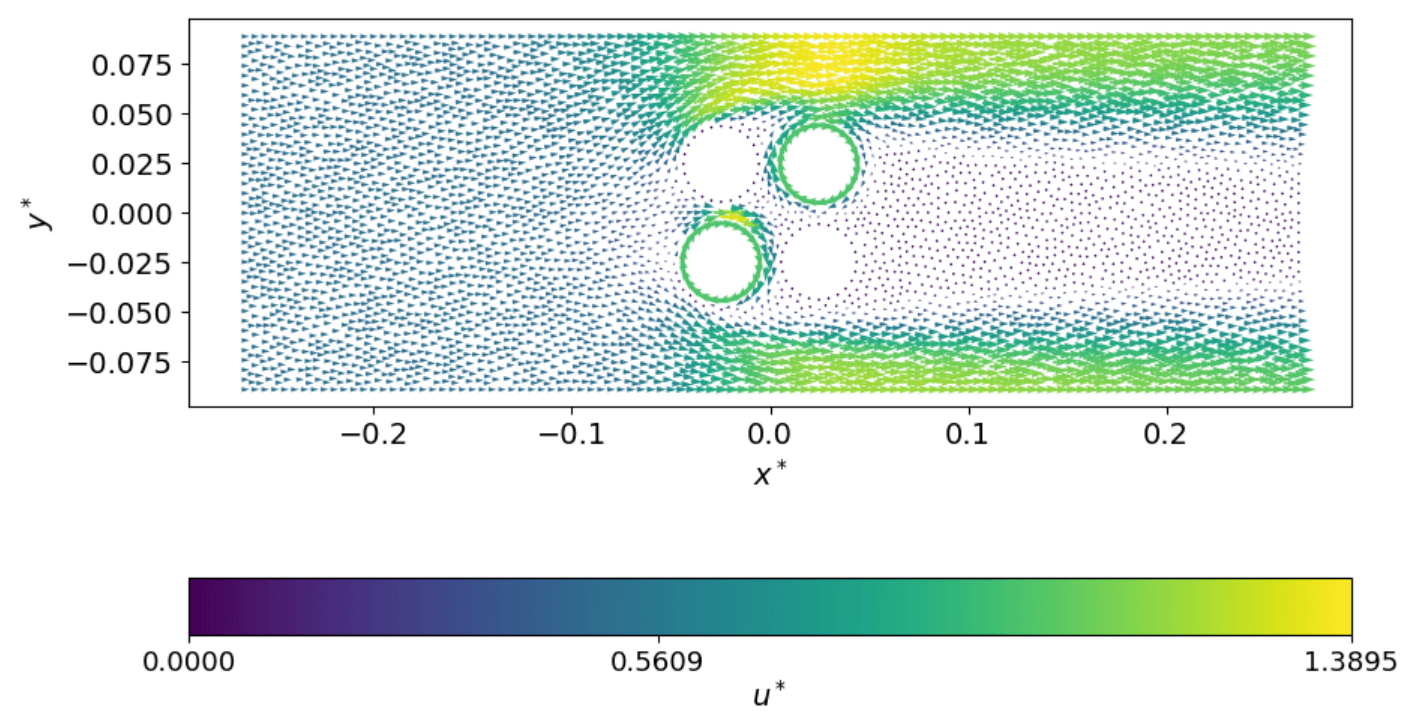

v) $R e_{\text {ent }}=10$

Figure 4.8: Velocity field in the expanded four-roll mill with $\lambda=0$ and distinct entrance Reynolds number. 

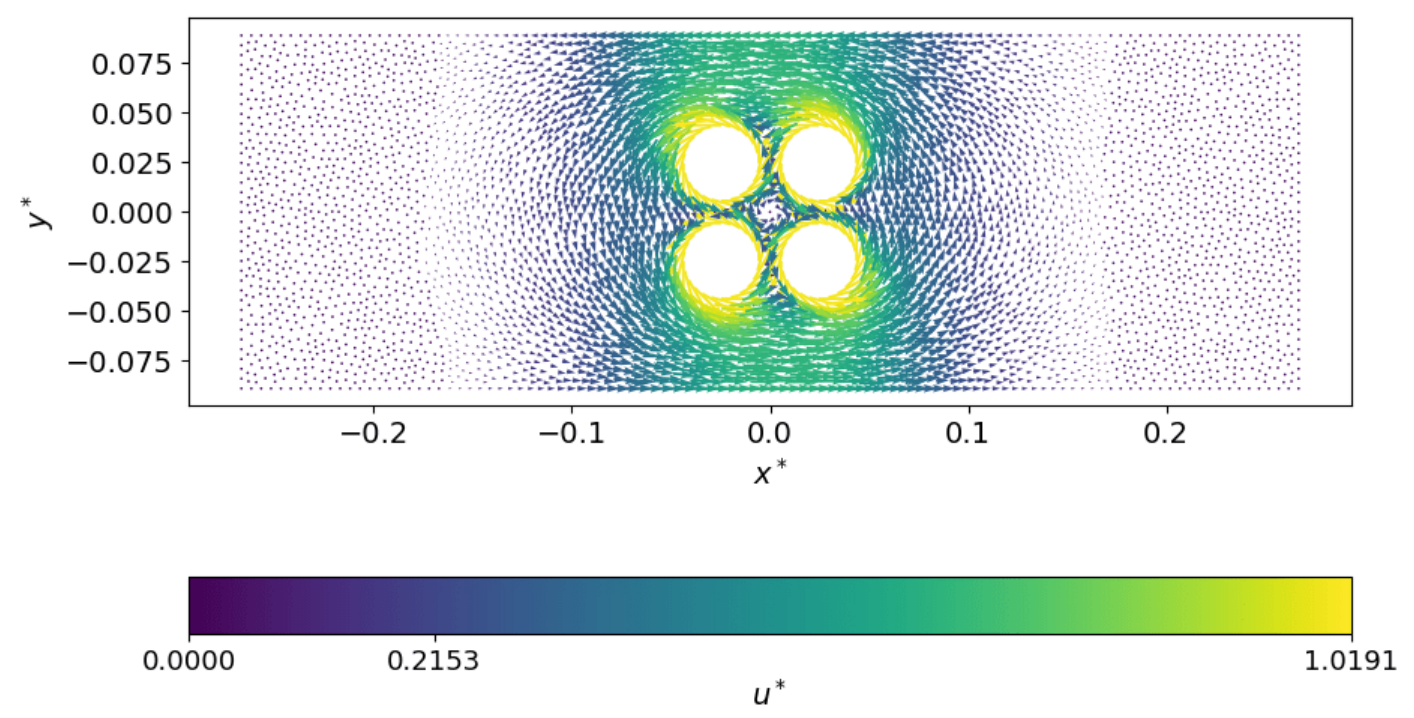

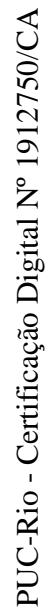

i) $R e_{\text {ent }}=0$
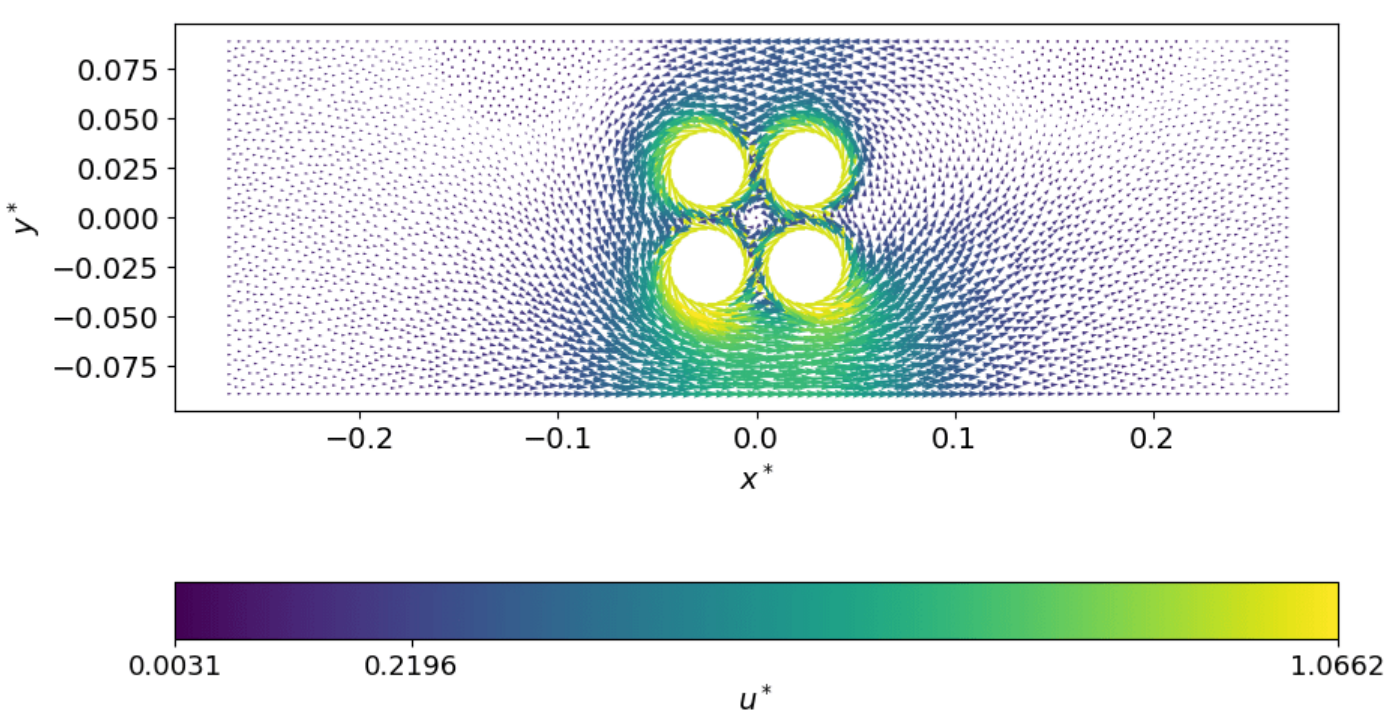

ii) $R e_{\text {ent }}=2$ 

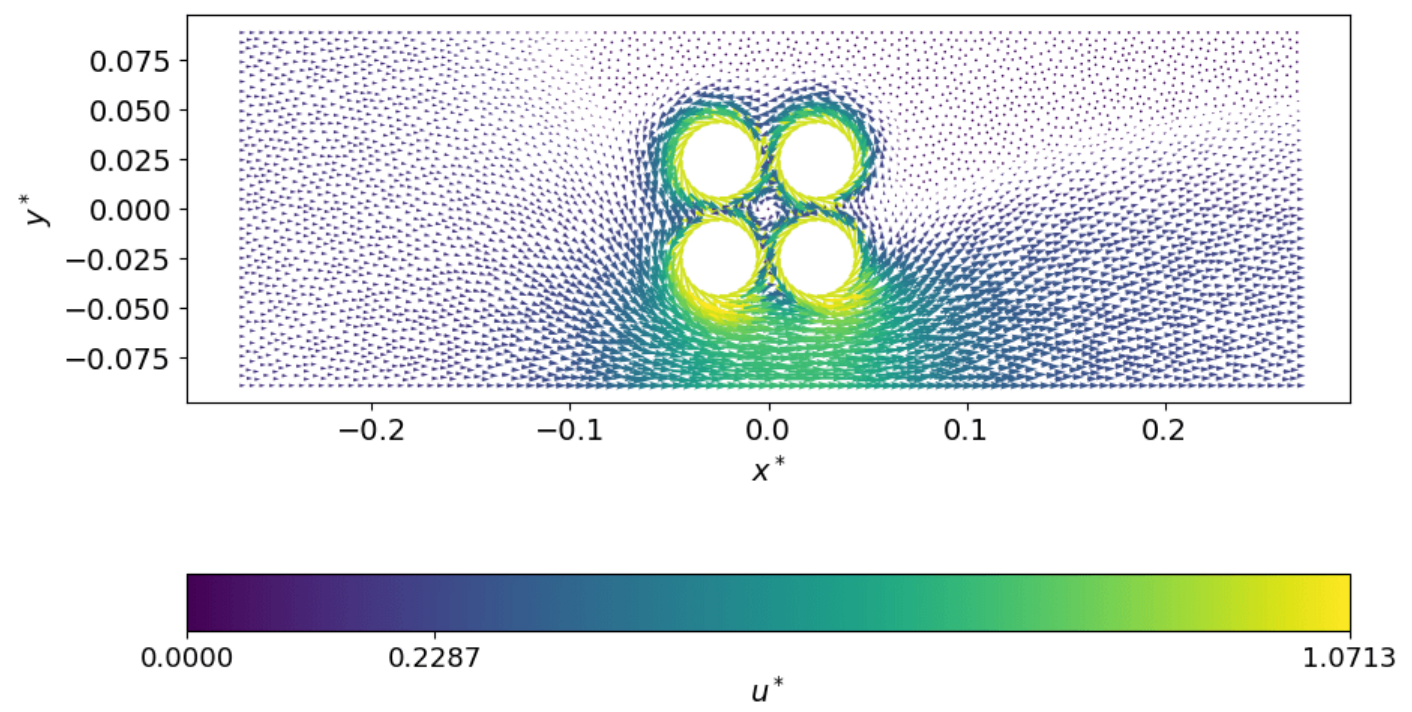

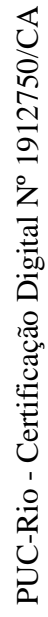

iii) $R e_{\text {ent }}=3$
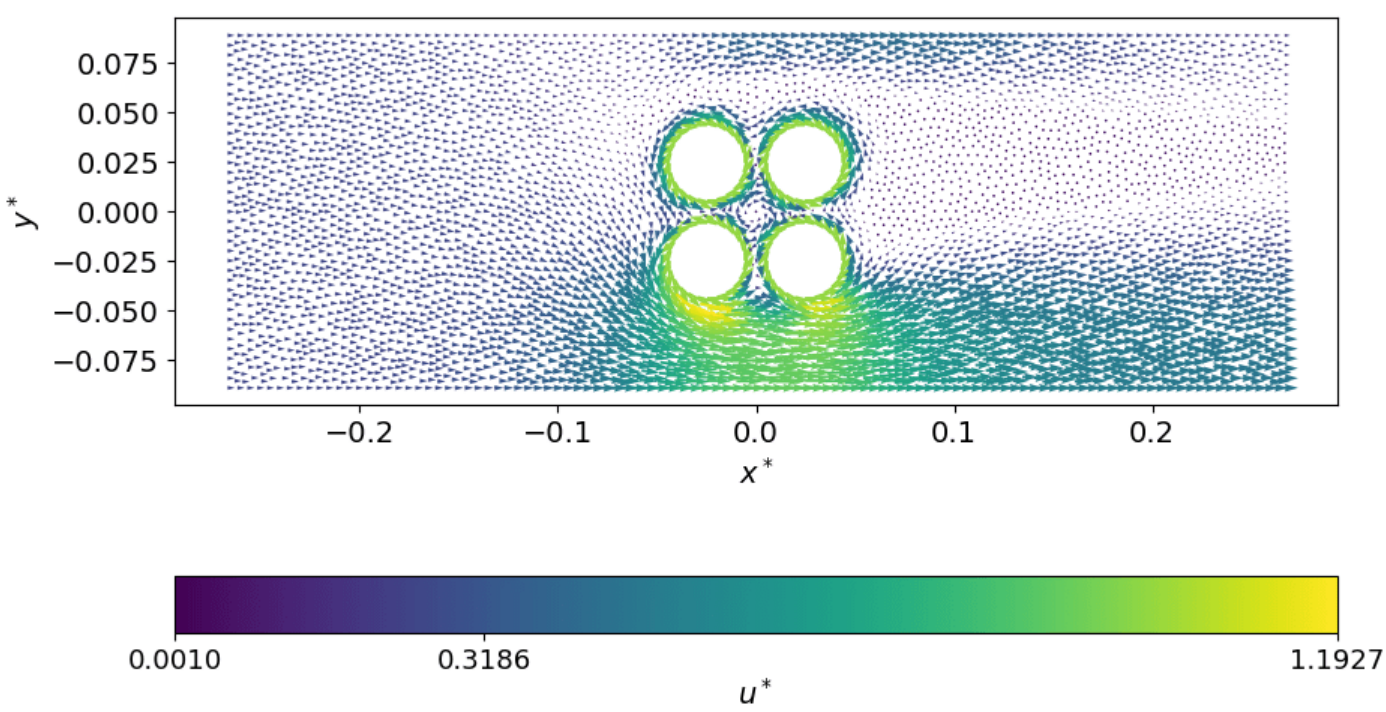

iv) $R e_{e n t}=5$ 

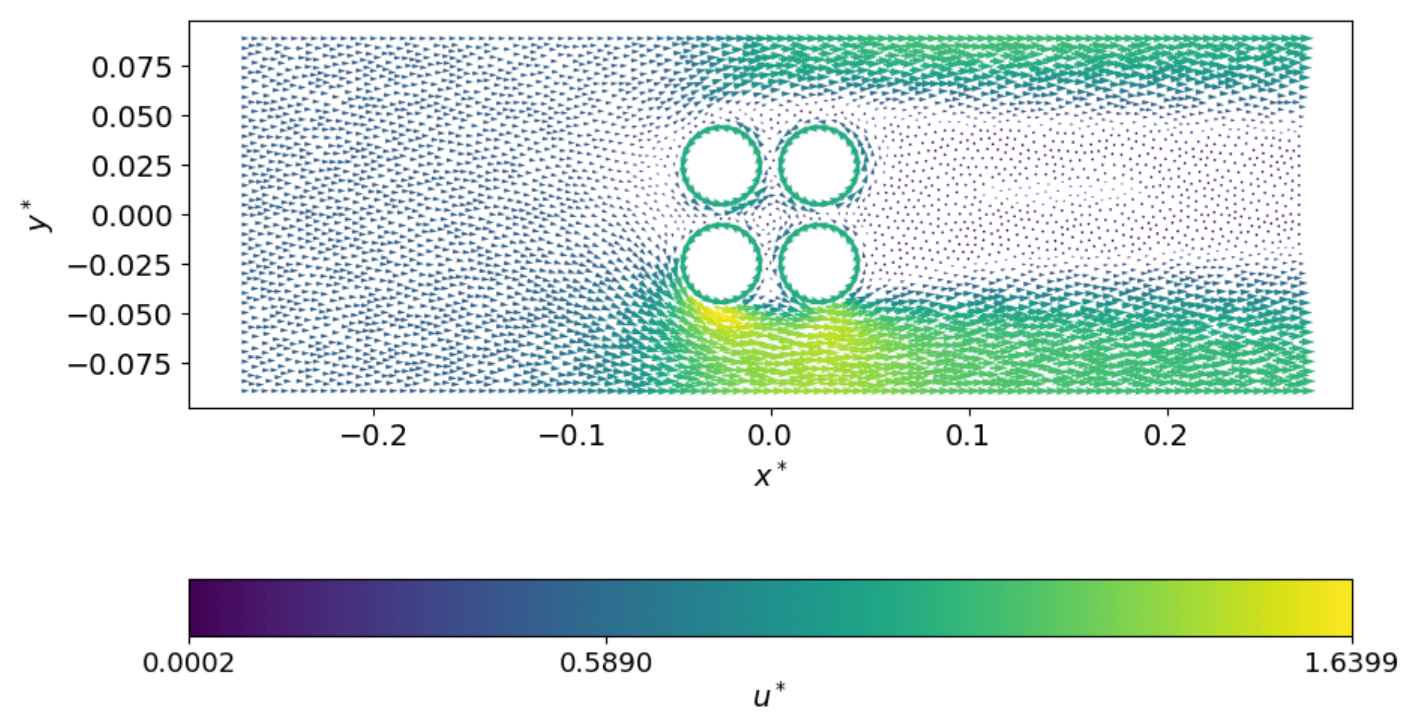

v) $R e_{\text {ent }}=10$

Figure 4.9: Velocity field in the expanded four-roll mill with $\lambda=-1$ and distinct entrance Reynolds number.

\subsection{2 \\ Streamlines}

Figures 4.10 and 4.11 exhibit the streamlines of each flow configuration on the expanded four-roll mill with $R e_{e n t}=0$ and $R e_{e n t}=5$ respectively. Comparing both meshes with a null inlet velocity, i.e. Figures 4.2 and 4.10, the outputs are very close in the internal region. Nonetheless, it is clear that the expanded four-roll mill enlarges the recirculation zones in cases $4.10 \mathrm{i}$ ) and $4.10 \mathrm{ii}$ ). In the specific case of $4.10 \mathrm{iii}$ ), a recirculation zone that did not exist in the classic mesh appeared right after the rollers in the expanded mesh. On the other hand, cases $4.10 \mathrm{iv}$ ) and $4.10 \mathrm{v}$ ) reveal closed streamlines both in internal and external regions. Both of them present a trend reciprocal with the cases $4.1 \mathrm{iv}$ ) and $4.1 \mathrm{v}$ ).

As already commented, the velocity fields with the $R e_{\text {ent }}=5$ become very similar and consequently the same happens for the streamlines. The general pattern of Figures 4.11 i) to $4.11 \mathrm{v}$ ) is typical of streamlines of a flow around static or rotating cylinder. The major difference among the cases displayed concerns the regions of re-circulation right after the rollers. It is worth mentioning that, in the specific case of the plot $4.11 \mathrm{i}$ ), the fluid particles access the internal region by the sideways and leave vertically exactly as desired from the application point of view in this configuration. 


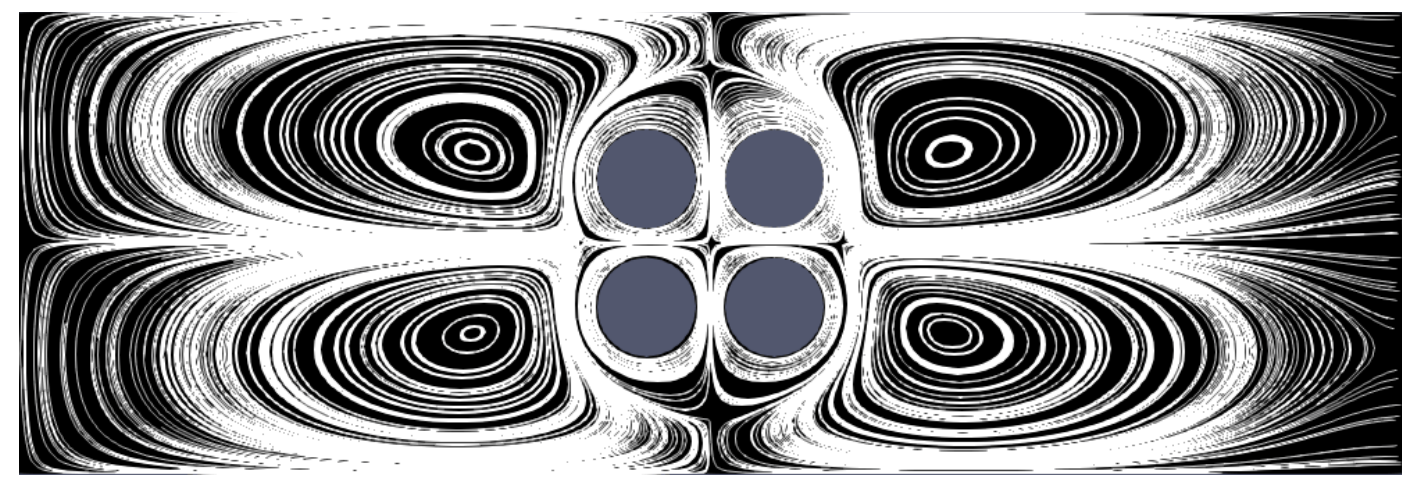

i) $\lambda=1$

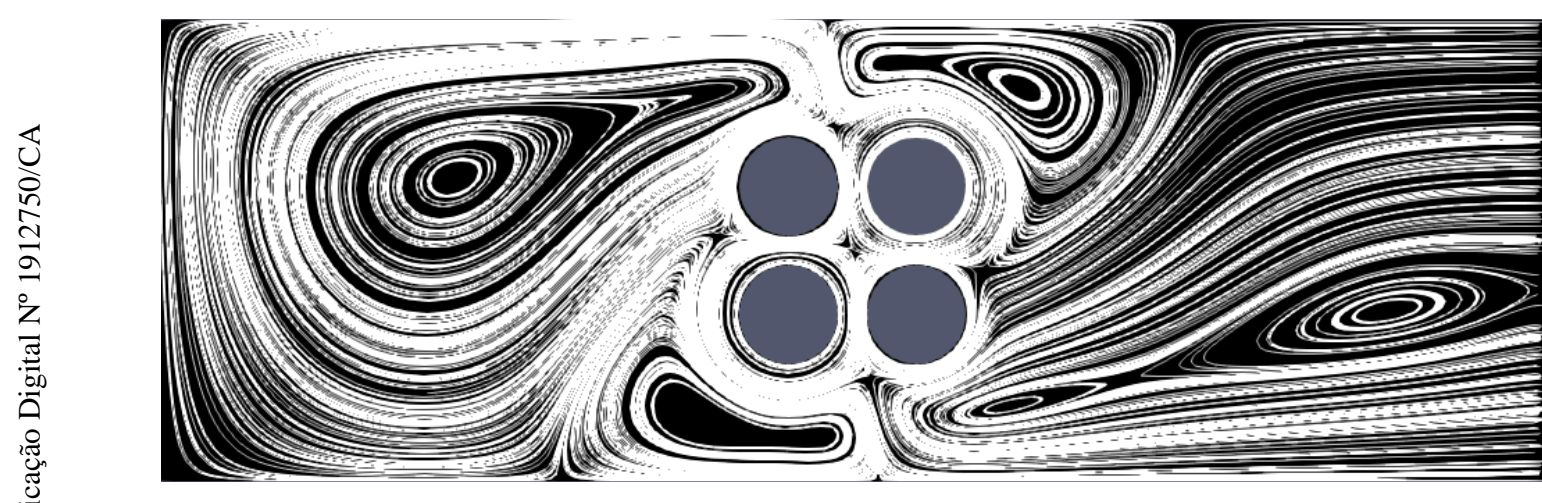

ii) $\lambda=0.5$

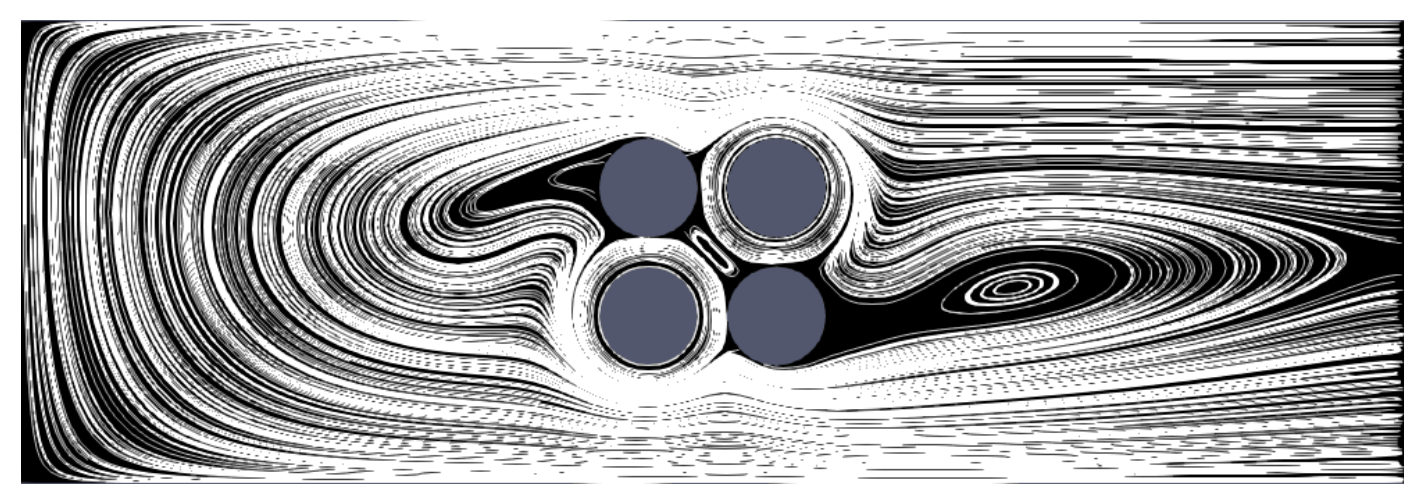

iii) $\lambda=0$ 


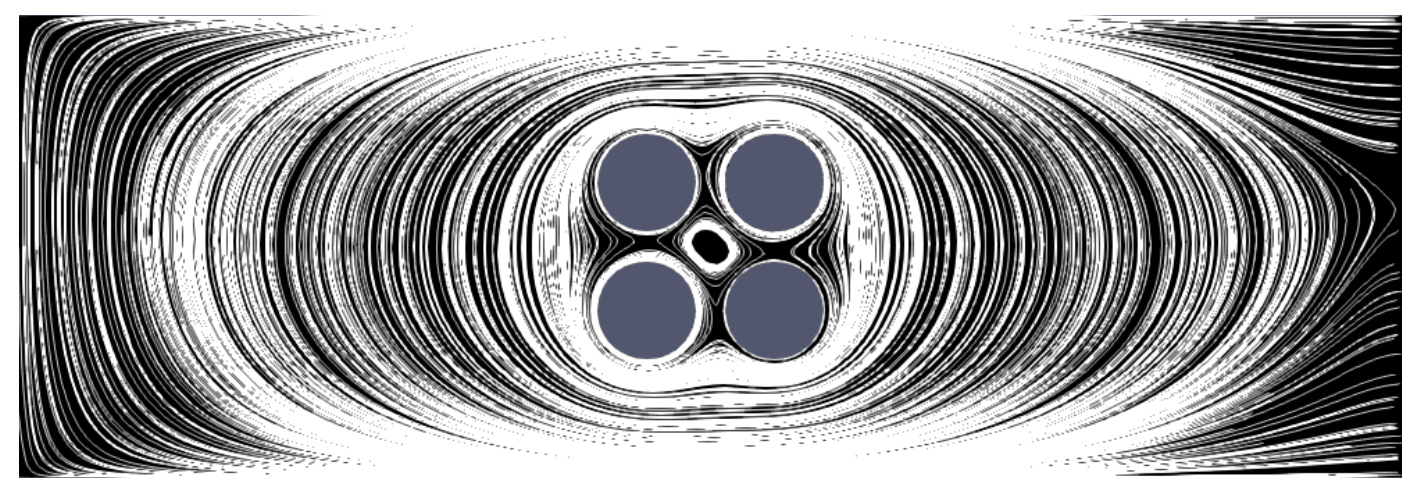

iv) $\lambda=-0.5$

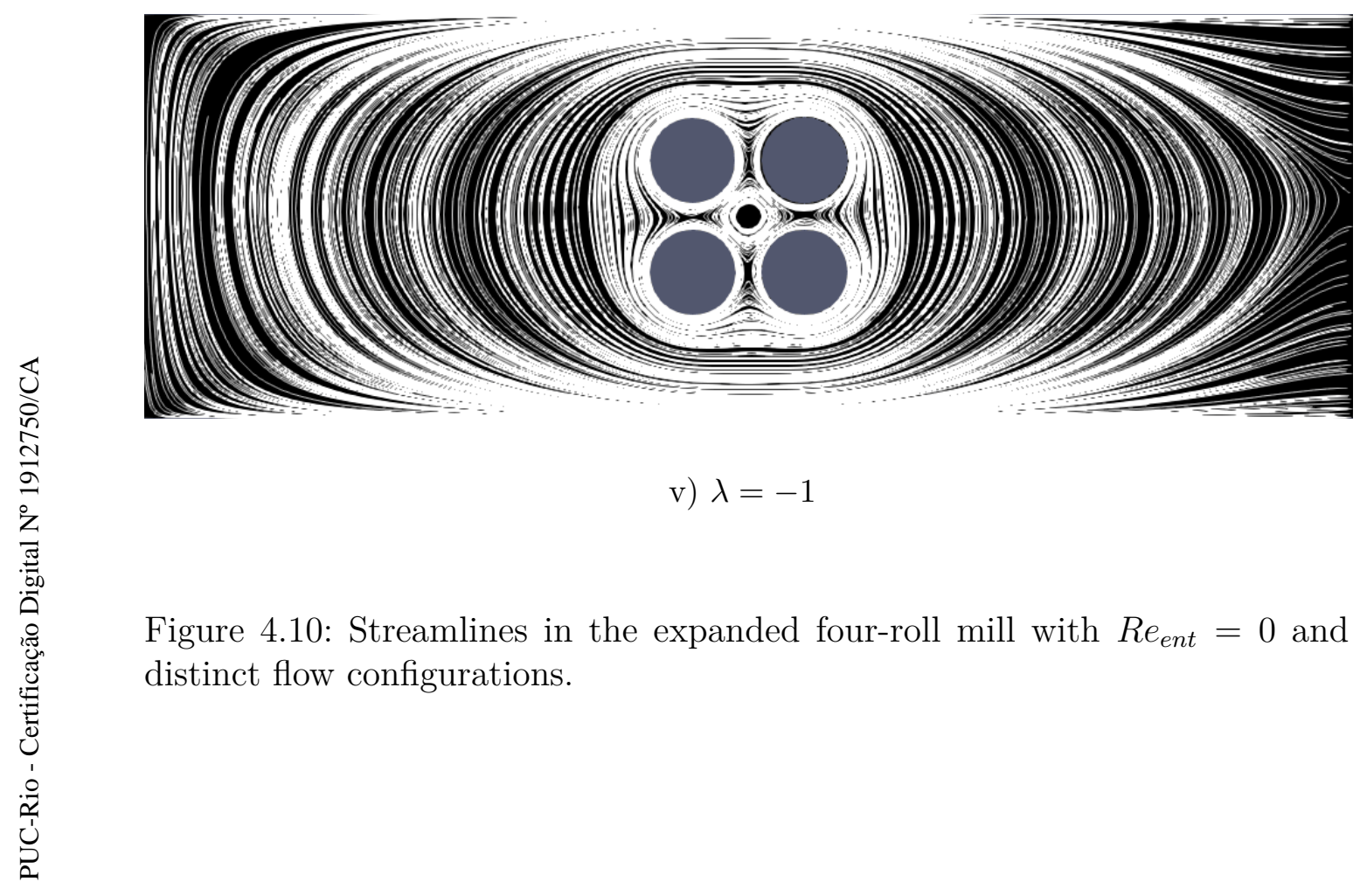




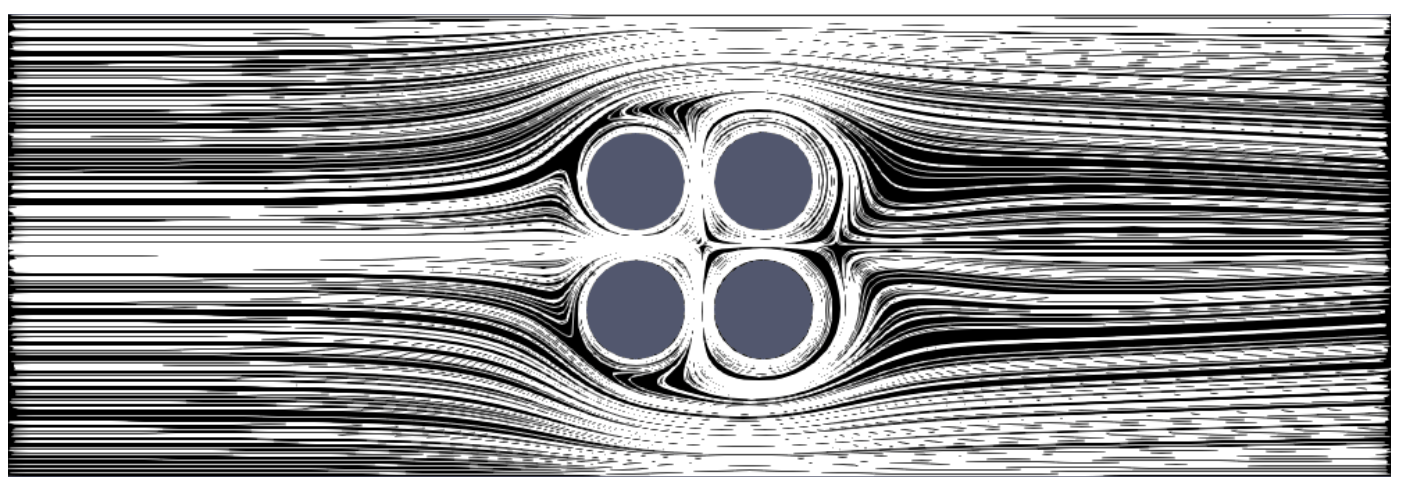

i) $\lambda=1$

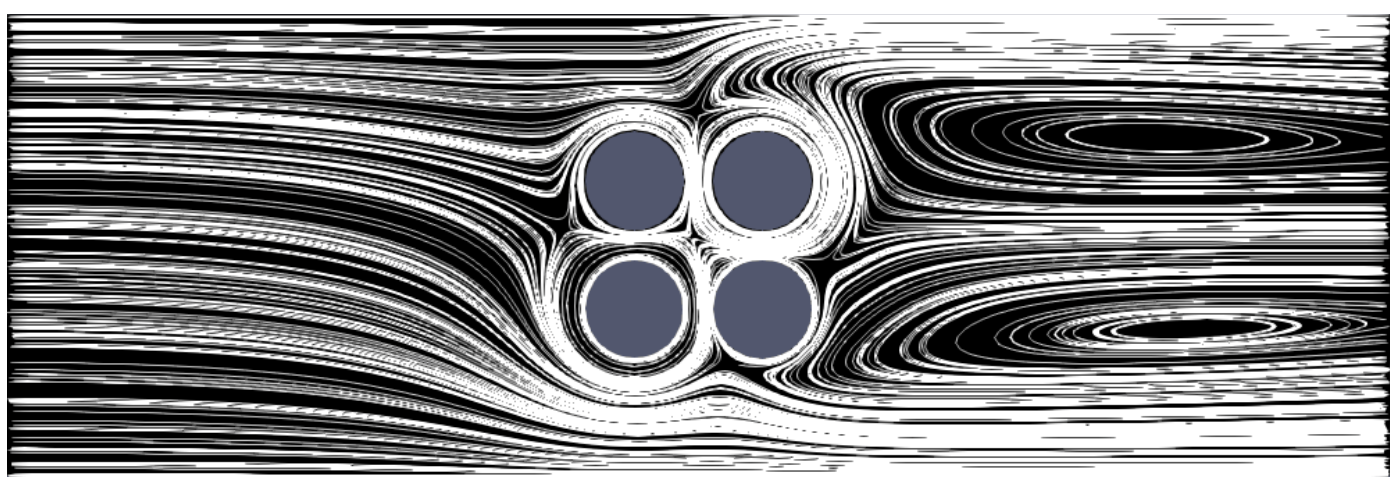

ii) $\lambda=0.5$

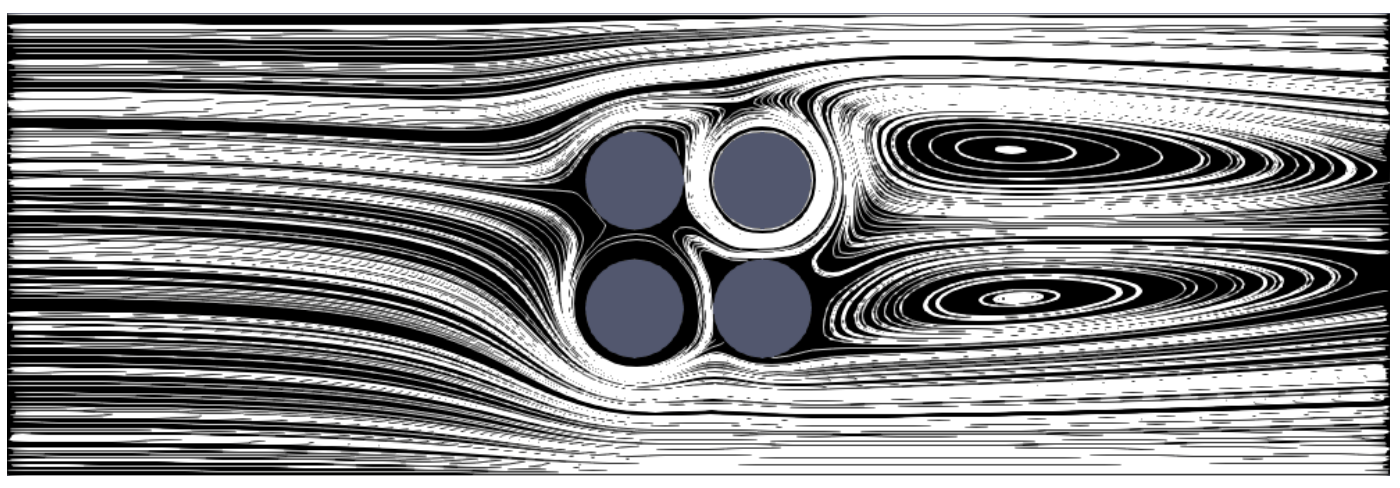

iii) $\lambda=0$ 


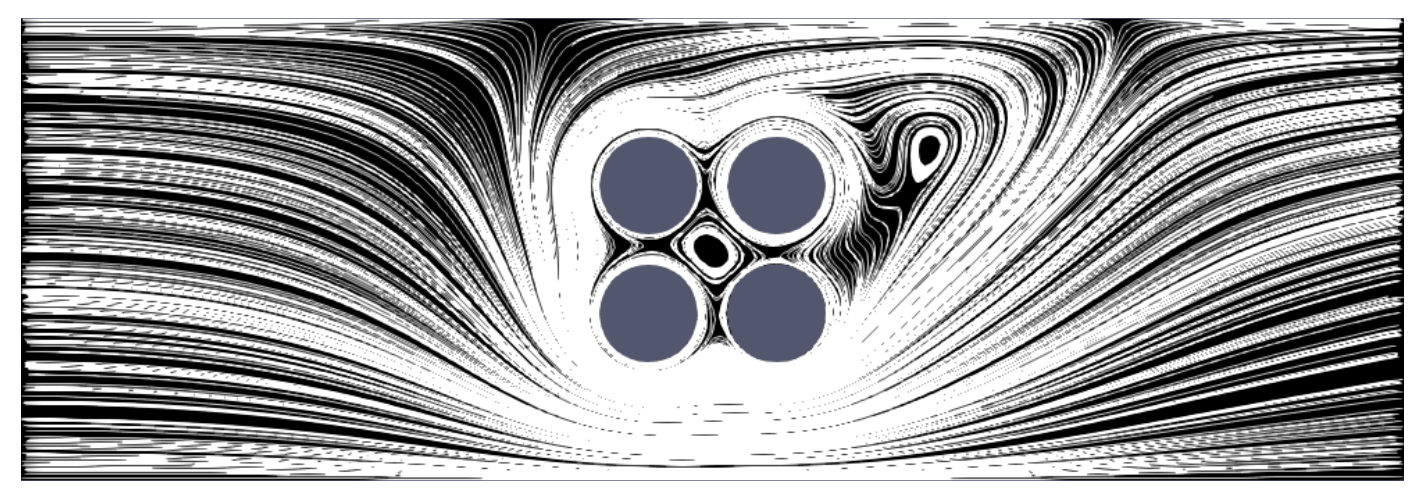

iv) $\lambda=-0.5$

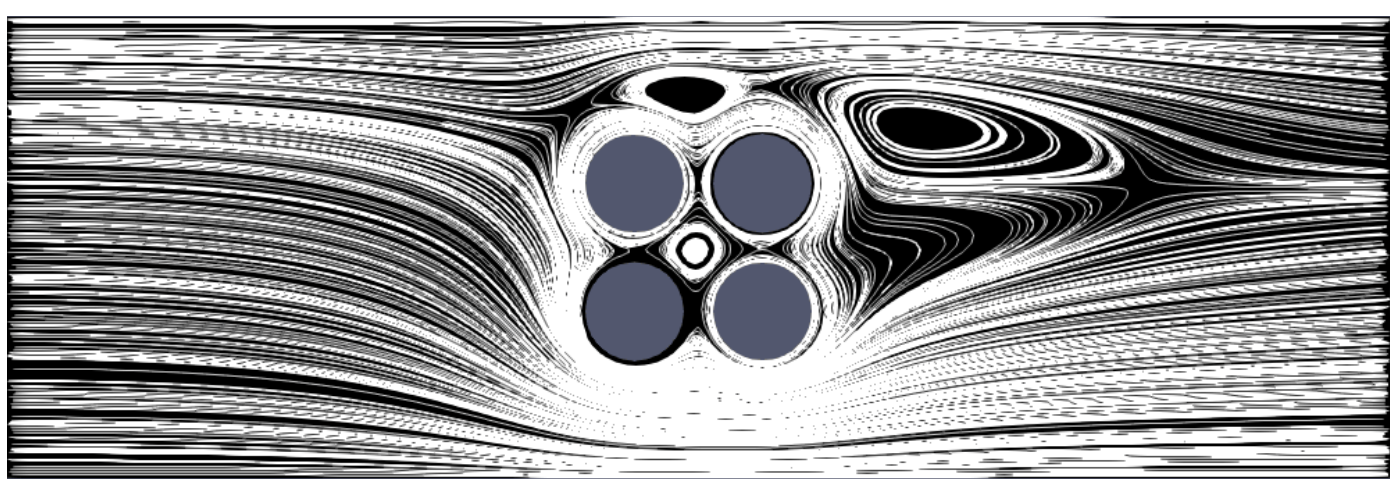

v) $\lambda=-1$

Figure 4.11: Streamlines in the expanded four-roll mill with $R e_{e n t}=5$ and distinct flow configurations.

\subsection{3}

\section{The normalized persistence-of-straining parameter fields}

Figure 4.12 shows the normalized persistence-of-straining parameter field with $R e_{e n t}=0$ and different flow configurations. It is interesting to superpose these figures with the original results from the classic four-roll mill. Thus, analyzing the results in the range of $-l \leq x \leq l$ for each flow configuration, they are almost the same as the ones for the classic four-roll mill presented in Figure 4.3. Outside this range, the results show different flow classifications. Near to the "West" and "East" borders, all the flow configurations present a mix of every single type of classifications. Therefore, it is clear that the use of the four-roll mill as an mechanism to expose the fluids to different kinds of flow is efficient. Specifically near the "West" border all the configurations display a small entrance region of simple shear flow. Despite the fact that $R e=0$, this shear region occurs due to the velocities of the rollers. 


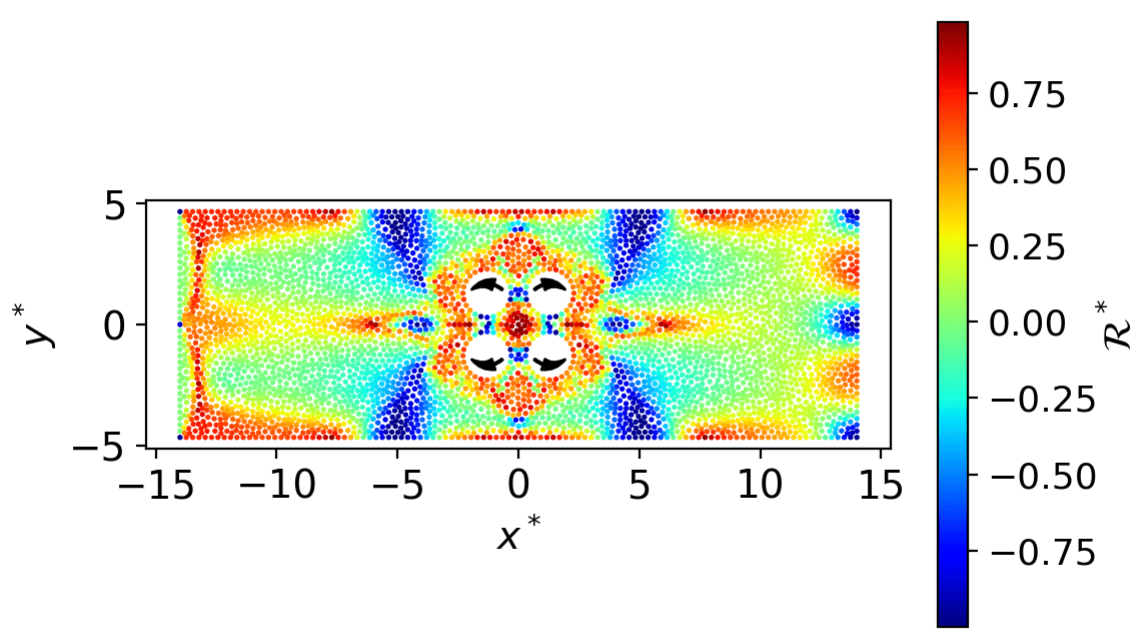

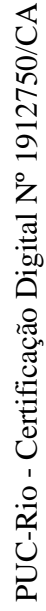

i) $\lambda=1$

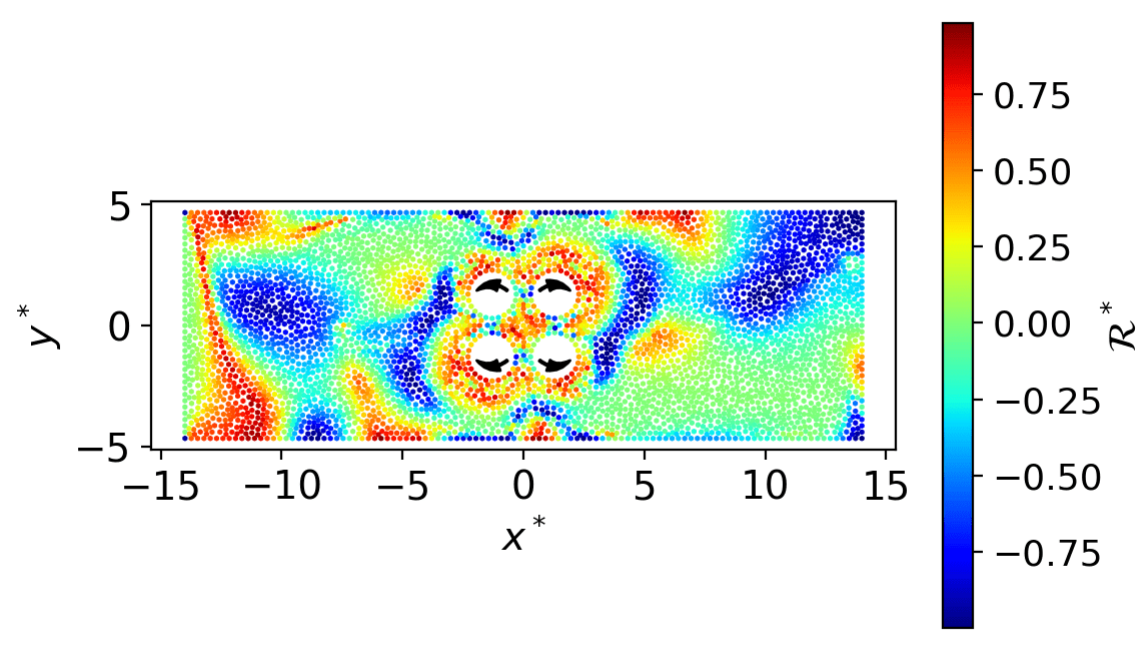

ii) $\lambda=0.5$ 


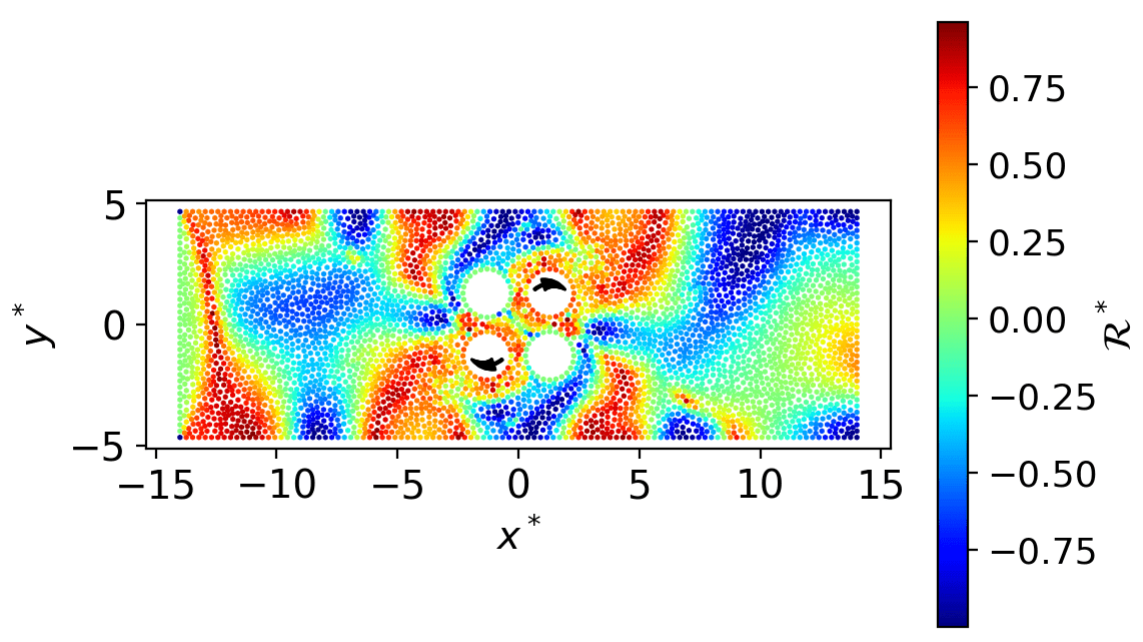

iii) $\lambda=0$

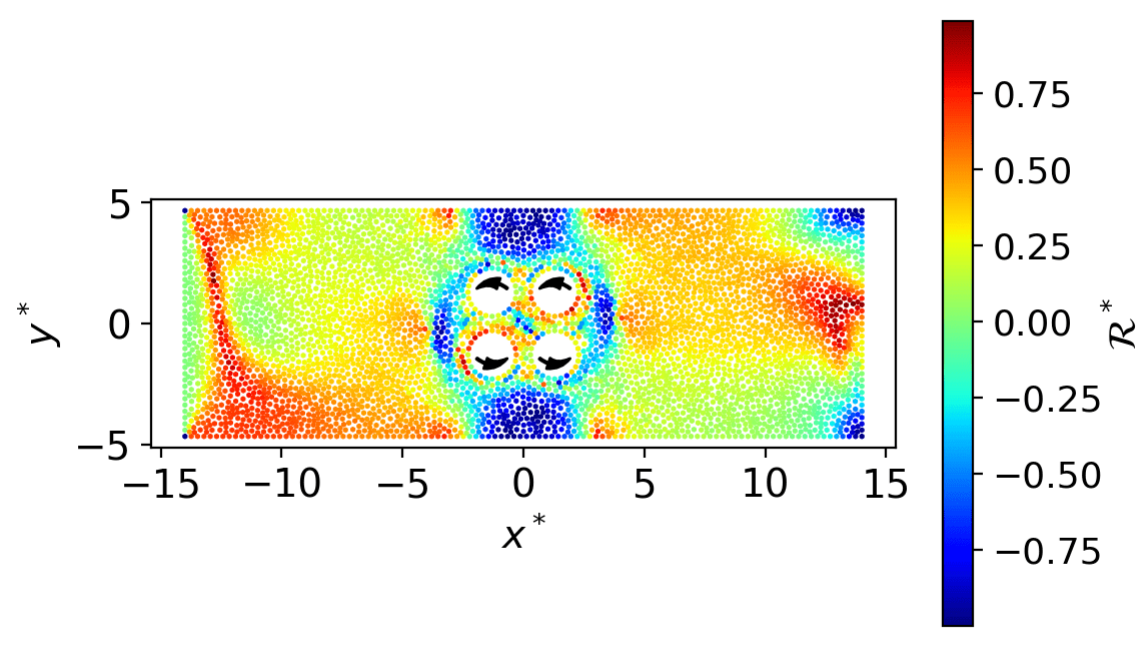

iv) $\lambda=-0.5$ 


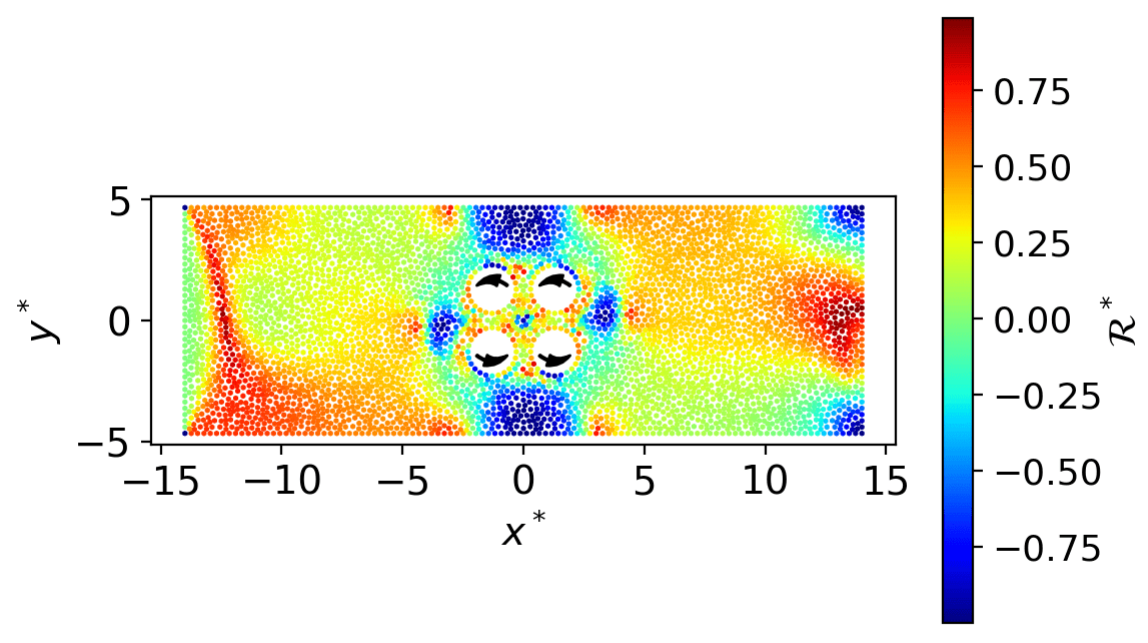

v) $\lambda=-1$

Figure 4.12: $\mathcal{R}^{*}$ field in the expanded four-roll mill with $R e_{\text {ent }}=0$ and distinct flow configurations.

The normalized persistence-of-straining parameter field with $R e_{e n t}=5$ and distinct $\lambda$ parameters, in turn, are indicated in Figure 4.13. Due to the fact that the results on each flow configuration converge for a solution close to the flow around a rotating cylinder, a clear pattern is established. Near the inlet region ("West" border), there is a relevant region with rigid body motion. In the areas close to the rollers, there is a transition where besides the rigid body motion, shear flow is also present. Naturally, huge areas after the rollers are shear wakes. Thus, in these areas, the normalized persistence-of-straining parameter $\mathcal{R}^{*} \rightarrow 0$, representing simple shear flow. 


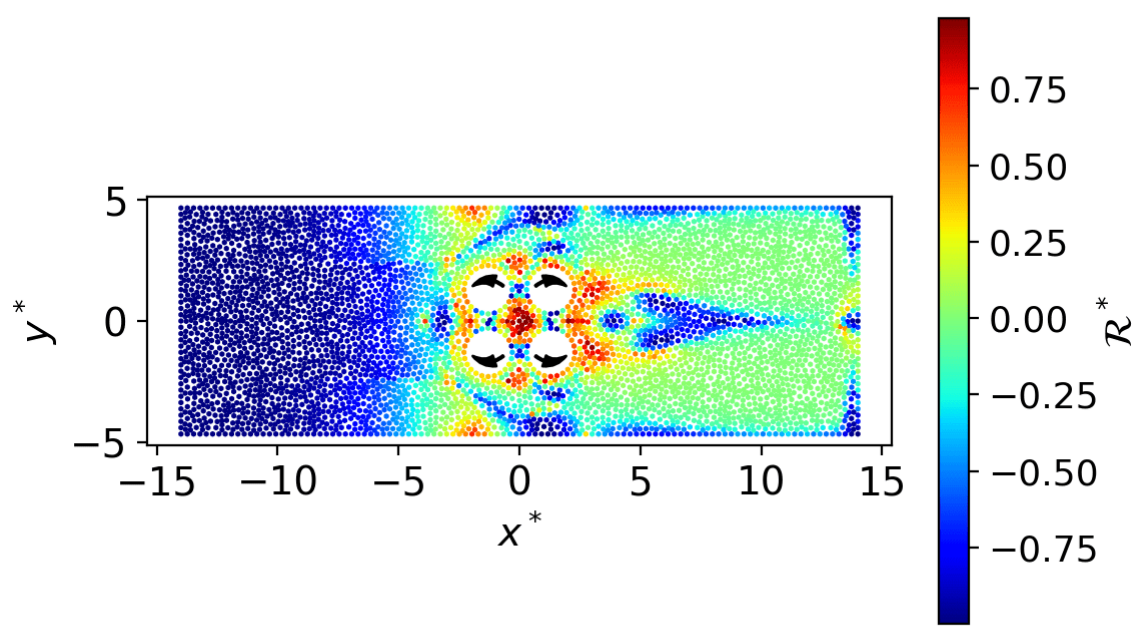

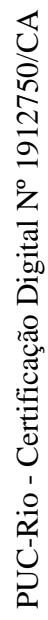

i) $\lambda=1$

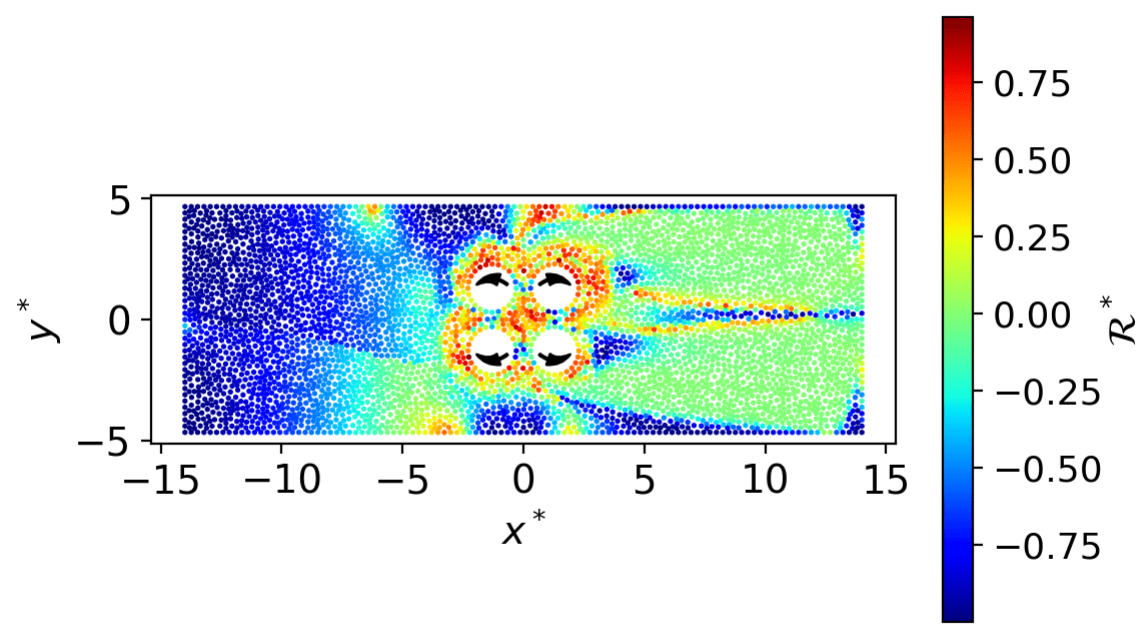

ii) $\lambda=0.5$ 


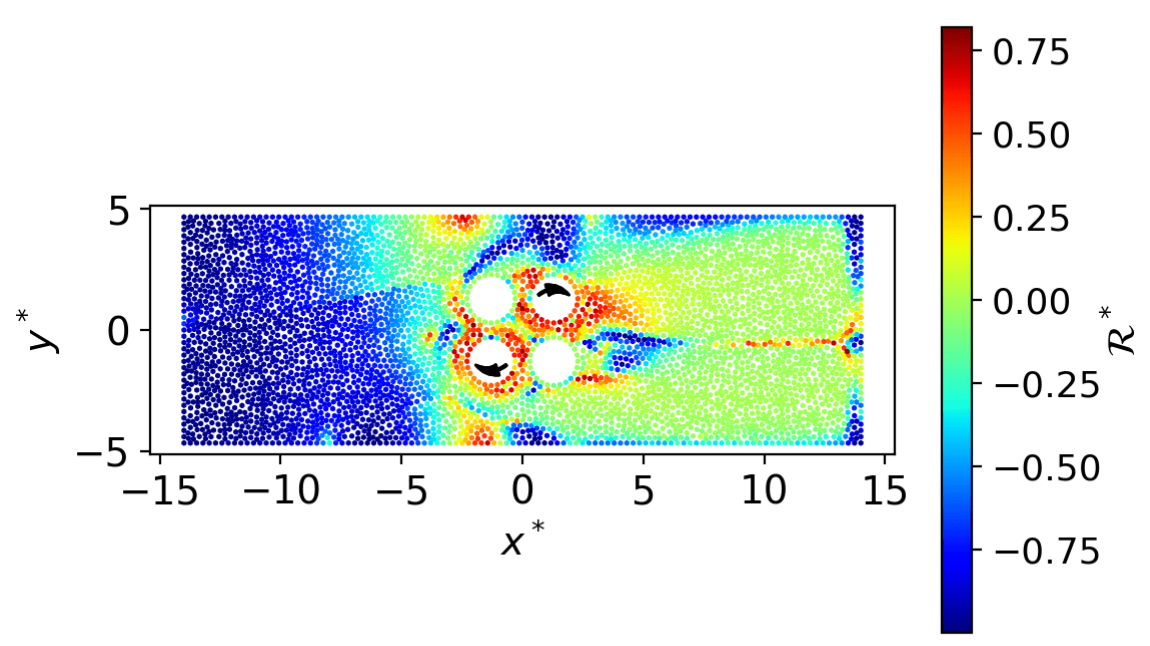

iii) $\lambda=0$

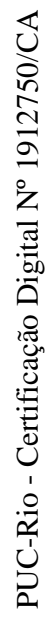

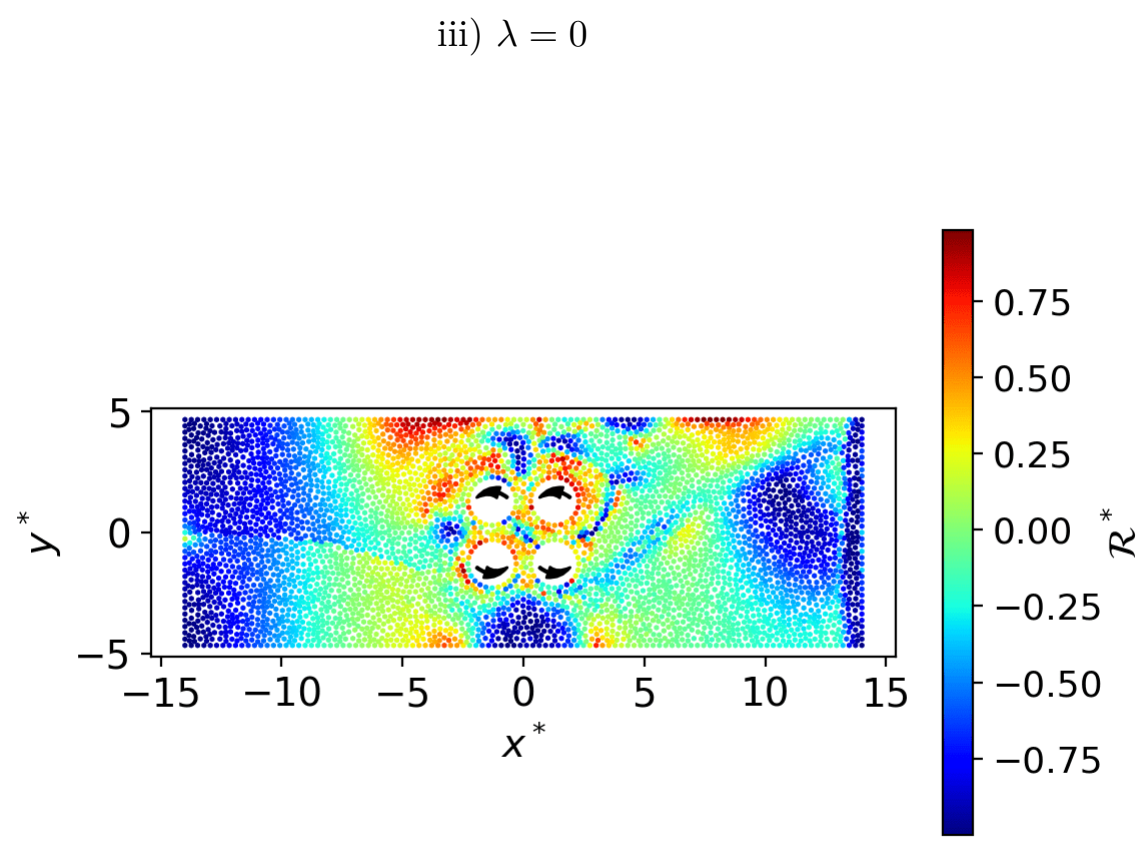

iv) $\lambda=-0.5$ 


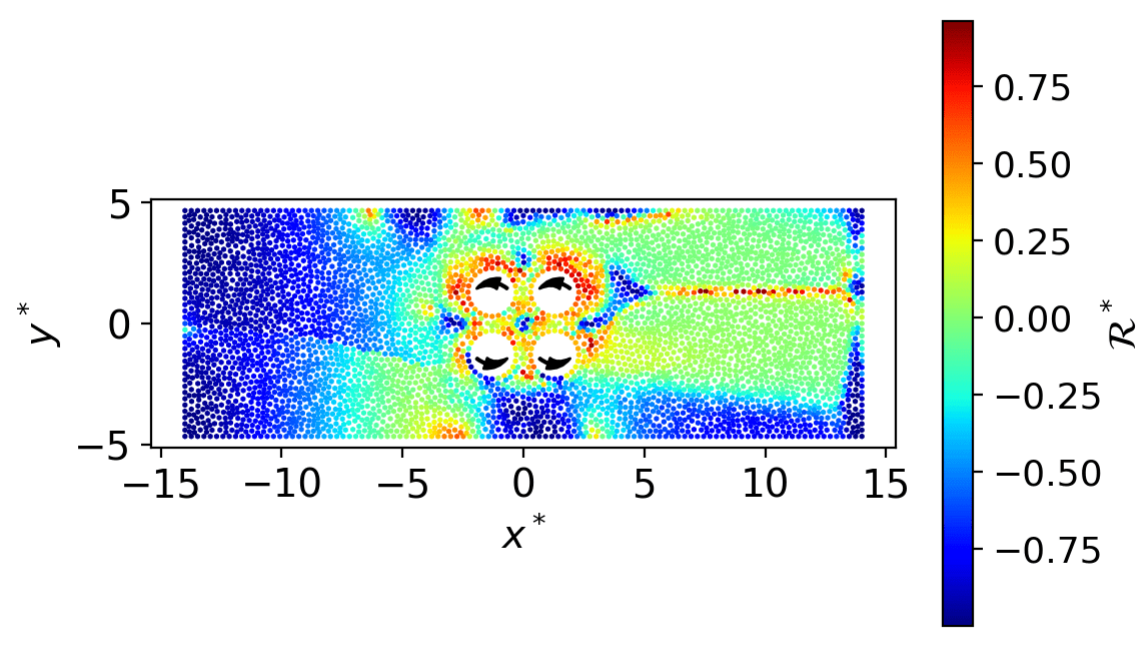

v) $\lambda=-1$

Figure 4.13: $\mathcal{R}^{*}$ field in the expanded four-roll mill with $R e_{\text {ent }}=5$ and distinct flow configurations.

\subsection{4}

\section{The modified persistence-of-straining parameter fields}

The results of modified persistence-of-straining parameter field for the expanded four roll mill are quite analogous from the ones of the classic fourroll mill. The same pattern is observed in which the increase of parameters $\alpha$ or $G$ are directly related to the increase of the presence of regions with extension, while the decrease of $G$ corresponds to more regions of rigid body motion, even with a non-null imposed velocity. Therefore, to avoid repetition, the decision was to only plot the modified persistence-of-straining parameter field for the flow configuration $\lambda=1$ both with $R e_{\text {ent }}=0$ and $R e_{\text {ent }}=5$, which are respectively displayed in Figures 4.14 and 4.15 . 


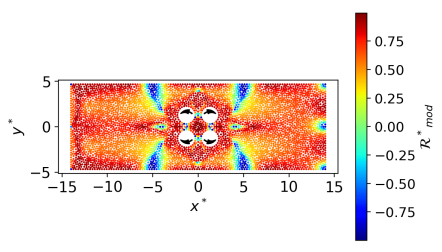

i) $G=4 ; \alpha=0$

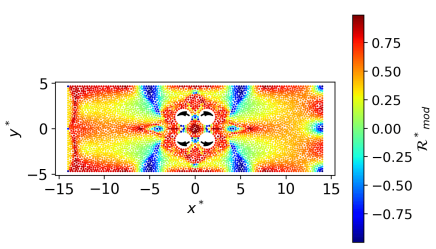

iv) $G=2 ; \alpha=0$

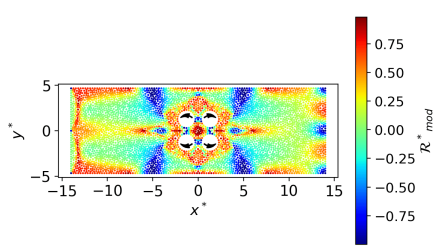

vii) $G=1 ; \alpha=0$

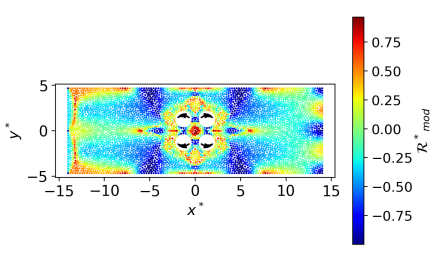

x) $G=0.5 ; \alpha=0$

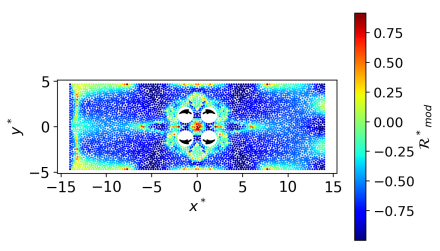

xiii) $G=0.2 ; \alpha=0$

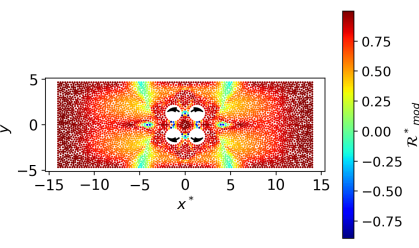

ii) $G=4 ; \alpha=0.2$

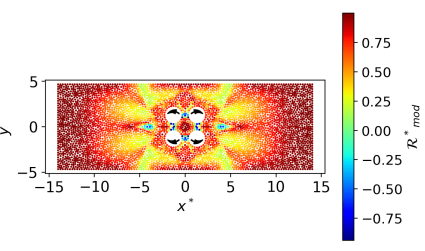

v) $G=2 ; \alpha=0.2$

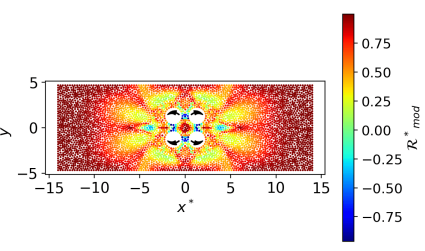

viii) $G=1 ; \alpha=0.2$

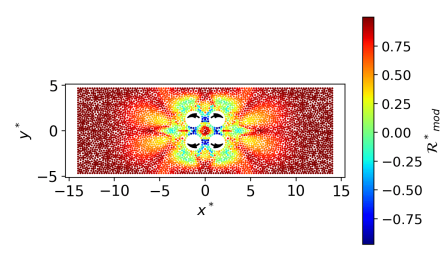

xi) $G=0.5 ; \alpha=0.2$

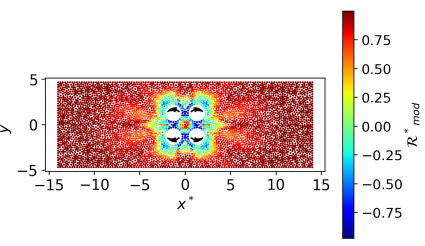

xiv) $G=0.2 ; \alpha=0.2$

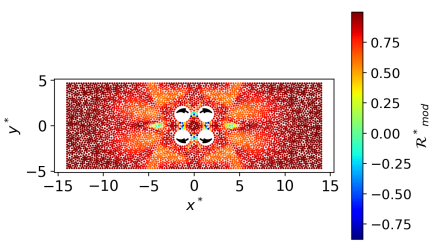

iii) $G=4 ; \alpha=0.5$

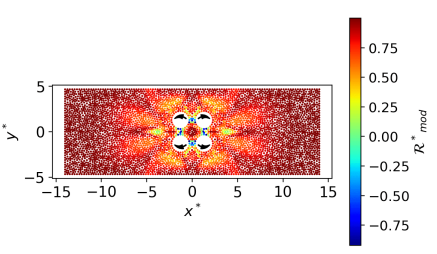

vi) $G=4 ; \alpha=0.5$

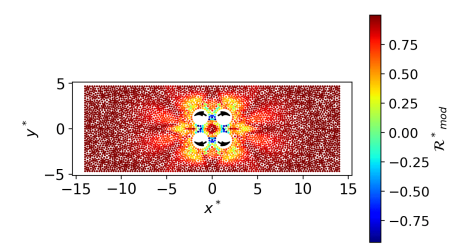

ix) $G=1 ; \alpha=0.5$

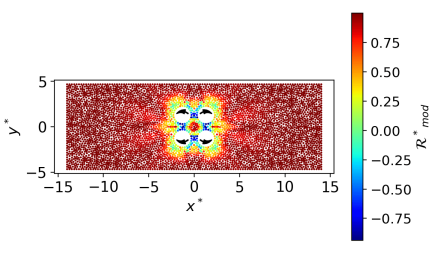

xii) $G=0.5 ; \alpha=0.5$

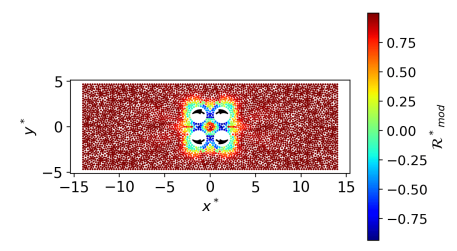

xv) $G=0.2 ; \alpha=0.5$

Figure 4.14: $\mathcal{R}^{*}{ }_{\text {mod }}$ field in the expanded four-roll mill with $\operatorname{Re}_{\text {ent }}=0, \lambda=1$, and distinct microelements. 


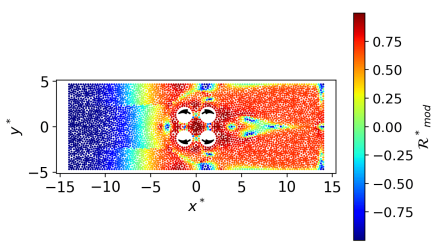

i) $G=6 ; \alpha=0$

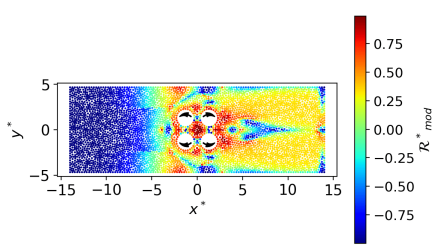

iv) $G=2 ; \alpha=0$

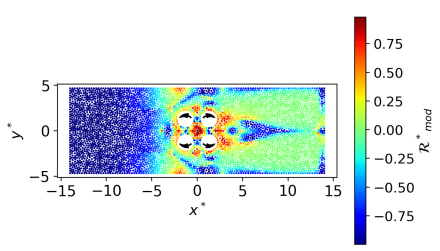

vii) $G=1 ; \alpha=0$

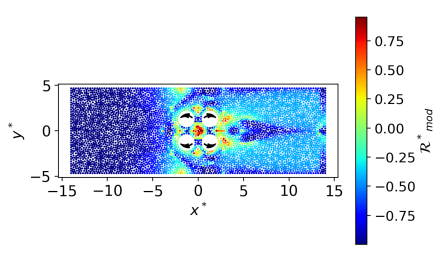

x) $G=0.4 ; \alpha=0$

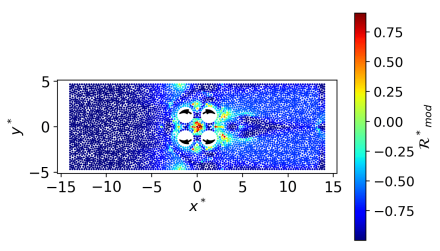

xiii) $G=0.2 ; \alpha=0$

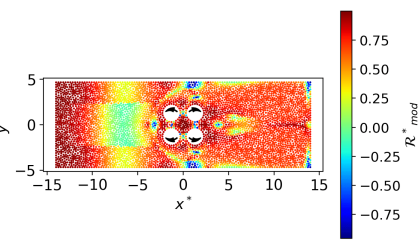

ii) $G=6 ; \alpha=0.2$

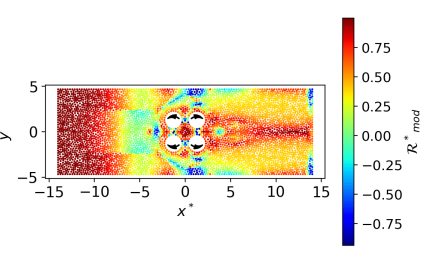

v) $G=2 ; \alpha=0.2$

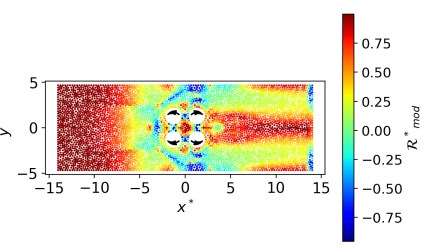

viii) $G=1 ; \alpha=0.2$

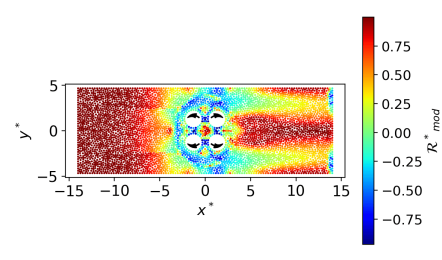

xi) $G=0.4 ; \alpha=0.2$

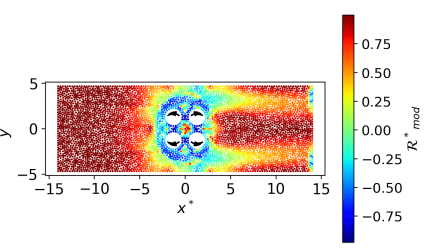

xiv) $G=0.2 ; \alpha=0.2$

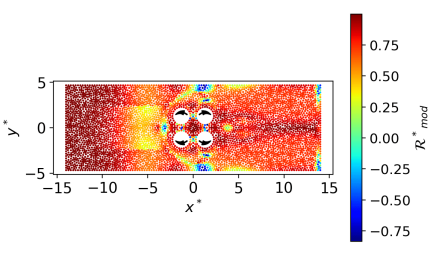

iii) $G=6 ; \alpha=0.5$

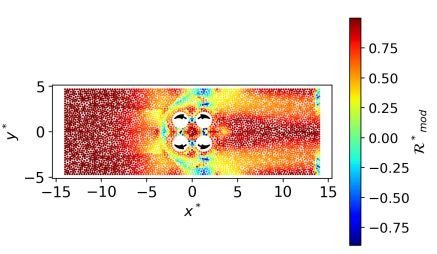

vi) $G=2 ; \alpha=0.5$

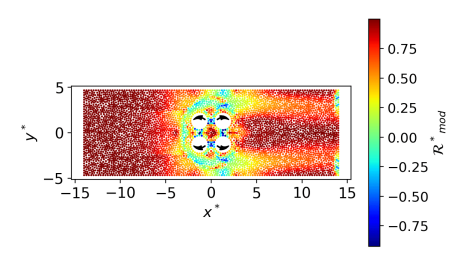

ix) $G=1 ; \alpha=0.5$

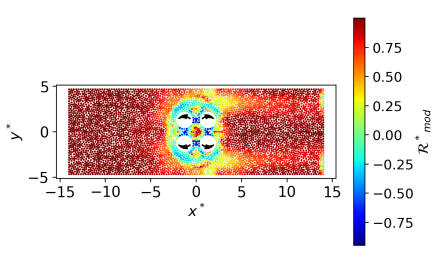

xii) $G=0.4 ; \alpha=0.5$

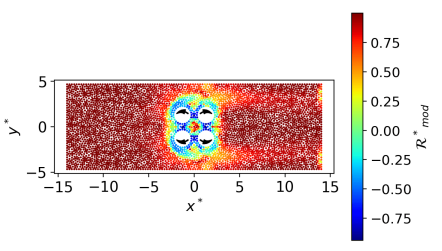

xv) $G=0.2 ; \alpha=0.5$

Figure 4.15: $\mathcal{R}^{*}{ }_{\text {mod }}$ field in the expanded four-roll mill with $R e_{\text {ent }}=5, \lambda=1$, and distinct microelements. 


\subsection{5}

\section{Pathline and deformation of microelements}

A few examples were selected to illustrate the deformation of microelements corresponding to the dispersed phase. First, four distinct microelements with different values of $G$ and $\alpha$ were considered. These cases lead to distinct modified persistence-of-straining parameter fields. Additionally, for each one of these cases, the microelements were initially placed (at $t^{*}=0$ ) at the "West" boundary at different vertical positions $y_{o}{ }^{*}$. Their respective pathlines are presented with the modified persistence-of-straining parameter fields in the background. The initial size of the vector is defined as $R^{1}=10^{-5}$ and the respective inclination $\theta^{1}=\pi / 4$. The fixed time step is $\Delta t=10^{-2}$.

Notice that it is not the aim of this work to define a criterion for drop breakup. The breakage is associated with large deformations. Therefore, the code is interrupted whenever the microelement reaches some assigned maximum deformation. Otherwise, the microelement will just reach the "East" boundary in order to stop the code. The drop is considered to be an ellipsoid of axis $a_{1}, a_{2}$, and $a_{3}$. The vector $\boldsymbol{R}$ is aligned with the direction of $a_{1}$. Thus, the deformation analyzed is exclusively in this specific direction. For simplicity, it is assumed that $a_{2}=a_{3}$ and consequently the volume of the drop is $V=\pi a_{1} a_{2}{ }^{2}$. Considering that the volume of the drop is conserved, the resistance of the vector $\boldsymbol{R}$ is higher to compression than to elongation. The compression of the direction of $a_{1}$ results in the elongation of the other two directions. The maximum deformation will be associated with $R^{*}=R^{*}{ }_{c r i t}$. Thus, the drop is assumed to break if $R^{*} \geq R^{*}{ }_{\text {crit }}$ or $R^{*} \leq \frac{1}{{R^{*}{ } r i t}^{2}}$.

Since the flow configuration $\lambda=-1$ does not facilitate the access of the microelements into the internal region, the microelement deformation is analyzed for $\lambda=1$ and $\lambda=0$ only, both of them with $R e_{e n t}=5$. Figure 4.16 shows the same microelement placed at different vertical positions and their respective pathlines. The background is the modified persistence-of-straining parameter field and it is based on the choice of $G$ and $\alpha$ on each case. Notice that for the scenario in Figure 4.16 iv) where the rigid body motion is predominant each pathline reaches the outlet ("East" border) since the drop maximum deformation is not achieved. In the other cases, many microelements positioned in different vertical positions break up before reaching the "East" border mainly because of shear and extension. 


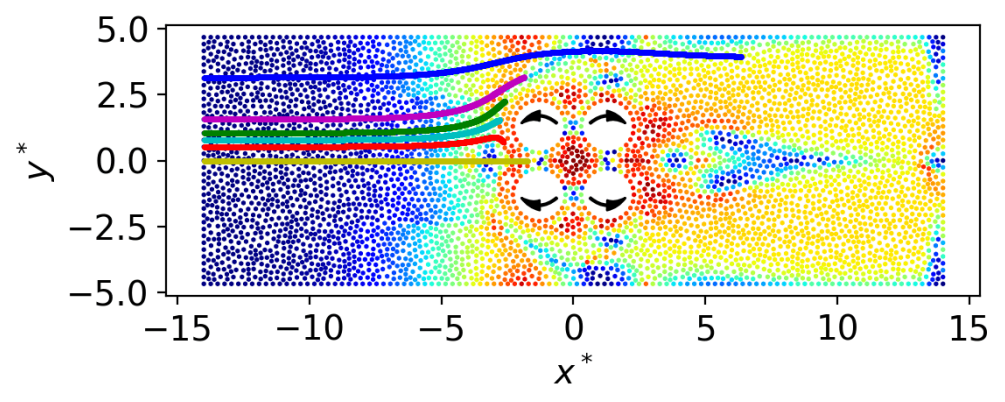

$$
\begin{aligned}
& \text { - } y_{0}{ }^{*}=3.15 \quad \text { } y_{0}{ }^{*}=0.79 \\
& \text { - } y_{0}{ }^{*}=1.57 \quad-y_{0}{ }^{*}=0.52 \\
& \text { - } y_{0}{ }^{*}=1.05 \quad y_{0}{ }^{*}=0
\end{aligned}
$$

i) $G=2, \alpha=0$

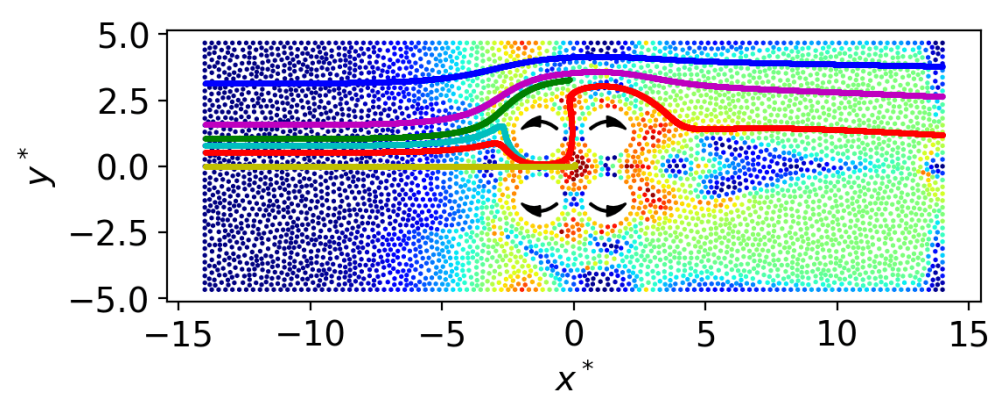

$$
\begin{array}{lll}
\text { - } y_{0}{ }^{*}=3.15 & \text { - } y_{0}{ }^{*}=0.79 \\
\text { - } y_{0}{ }^{*}=1.57 & \text { - } y_{0}{ }^{*}=0.52 \\
\text { - } y_{0}{ }^{*}=1.05 & \text { - } y_{0}{ }^{*}=0
\end{array}
$$

ii) $G=1, \alpha=0$

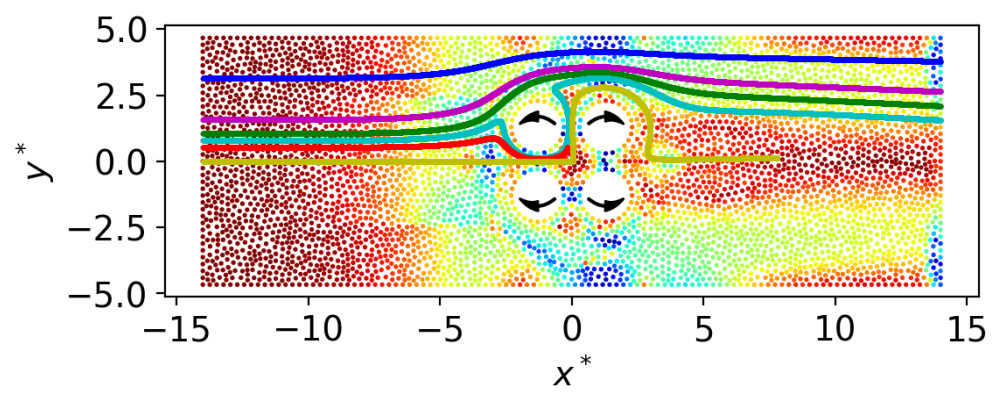

$$
\begin{array}{lll}
\text { - } y_{0}{ }^{*}=3.15 & \text { - } & y_{0}{ }^{*}=0.79 \\
\text { - } y_{0}{ }^{*}=1.57 & \text { - } & y_{0}{ }^{*}=0.52 \\
\text { - } y_{0}{ }^{*}=1.05 & \text { - } & y_{0}{ }^{*}=0
\end{array}
$$

iii) $G=1, \alpha=0.2$

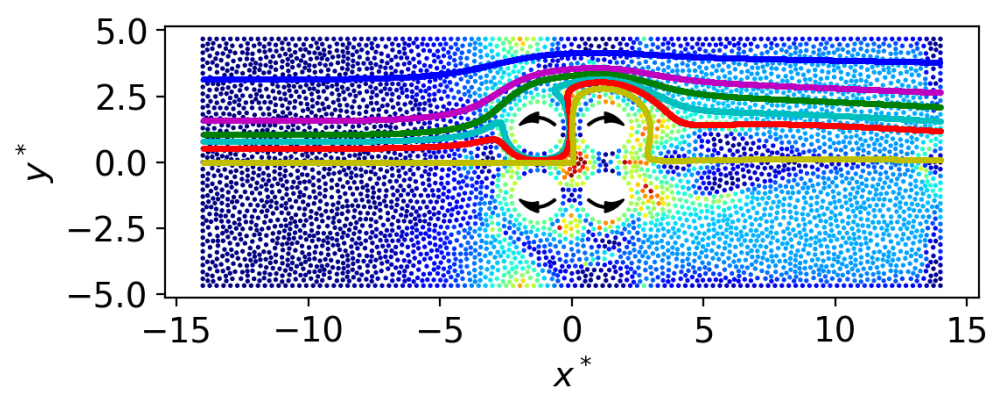

$$
\begin{array}{lll}
\text { - } y_{0}{ }^{*}=3.15 & \text { - } & y_{0}{ }^{*}=0.79 \\
\text { - } y_{0}{ }^{*}=1.57 & \text { - } & y_{0}{ }^{*}=0.52 \\
\text { - } y_{0}{ }^{*}=1.05 & \text { - } & y_{0}{ }^{*}=0
\end{array}
$$

iv) $G=0.4, \alpha=0$

Figure 4.16: Pathlines in the expanded four-roll mill with $R e_{e n t}=5, \lambda=1$, and distinct microelements placed at different initial vertical positions. 
At this point, the initial vertical position $y_{o}{ }^{*}=0$ is selected to illustrate some interesting mechanical behaviors of emulsions. Figure 4.17 gives the nondimensional position $x^{*}$ versus time $t^{*}$ and non-dimensional position $y^{*}$ versus time $t^{*}$ for $y_{o}{ }^{*}=0$, and with $\lambda=1$ and $R e_{e n t}=5$. Notice that the plots contain the same paths for both directions because the pathline depends only on the velocity field. The criterion of maximum deformation causes interruption at different times for each microelement, based on its properties. For instance, for the case $G=1$ and $\alpha=0$ represented on figure 4.17 iii), the code is interrupted right after the microelement reaches the origin of the domain, which corresponds to the center of the four-roll mill. Horizontal black dashed lines were plotted to highlight the $x^{*}$ position range which contains the internal region of the four-roll mill. In addition, three vertical yellow dashed lines were drawn to indicate when the inlet, middle and outlet positions of the internal region of the four-roll mill, i.e. the positions at $x^{*}{ }_{4 R M \_i n l e t}=-\frac{b+a}{a}$, $x^{*}{ }_{4 R M \_c e n t e r}=0$, and $x^{*}{ }_{4 R M \_o u t l e t}=\frac{b+a}{a}$, are reached. These specific markers are in fact reached (if the deformation is lower than the maximum) at the dimensionless times $t^{*}=51.12,55.29$, and 66.81. In the case represented in figure 4.17 i), i.e. $G=2$ and $\alpha=0$, the microelement reaches the inlet of the internal region at time $t^{*}=51.12$ and breaks right after at time $t^{*}=52.16$, never reaching the center.

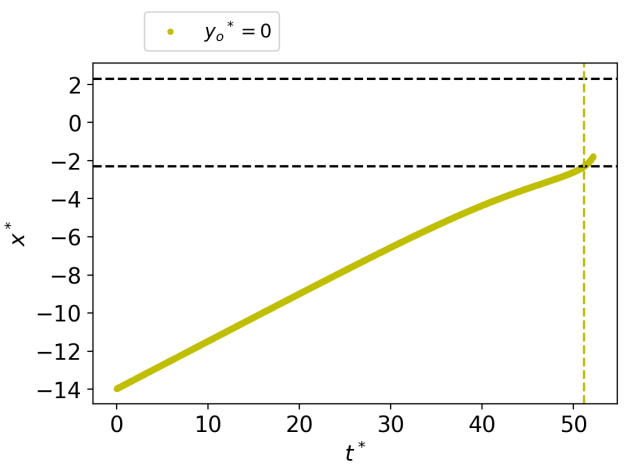

i) $G=2 ; \alpha=0$

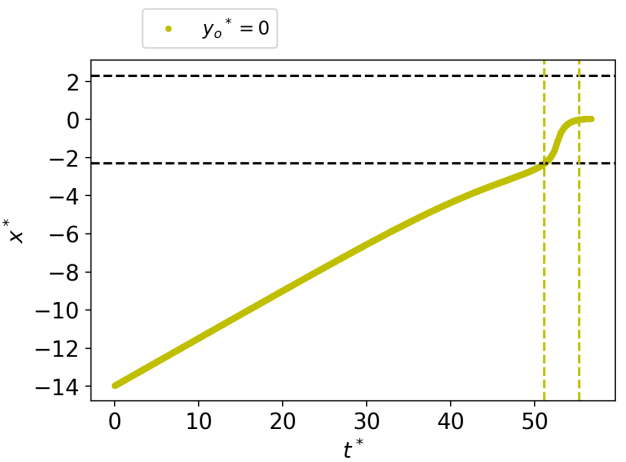

iii) $G=1 ; \alpha=0$

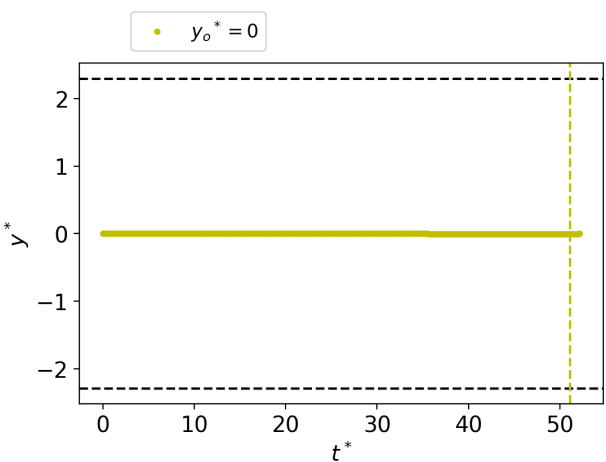

ii) $G=2 ; \alpha=0$

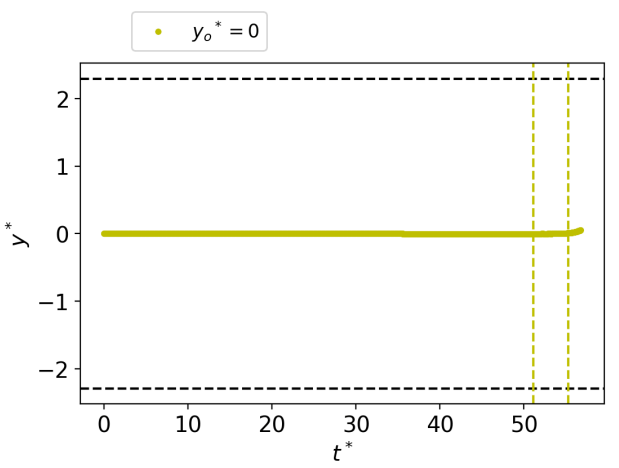

iv) $G=1 ; \alpha=0$ 

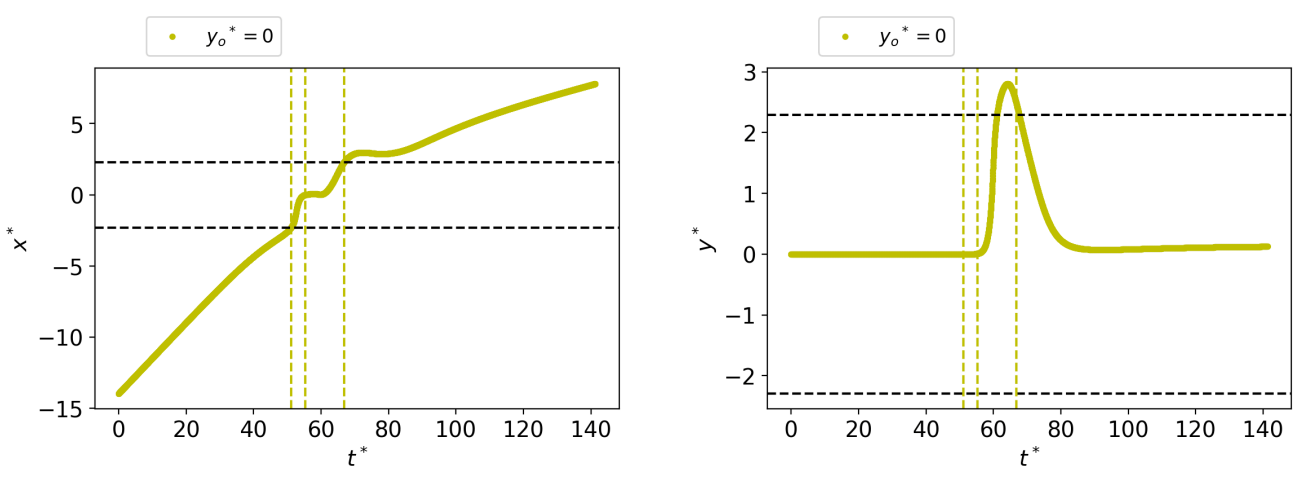

v) $G=1 ; \alpha=0.2$

vi) $G=1 ; \alpha=0.2$
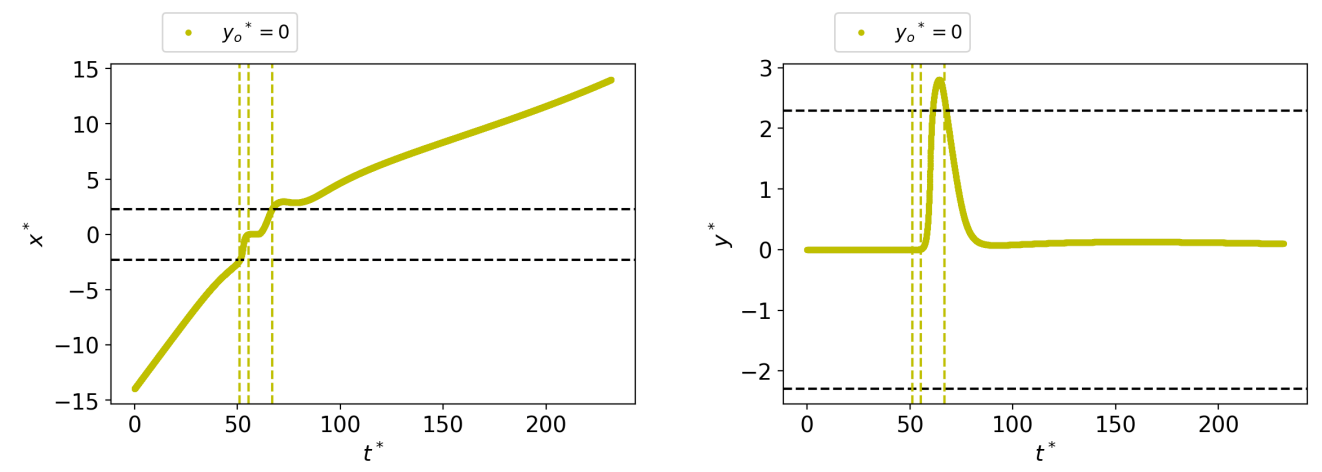

vii) $G=0.4 ; \alpha=0$

viii) $G=0.4 ; \alpha=0$

Figure 4.17: $x^{*}$ versus $t^{*}$ (left column) and $y^{*}$ versus $t^{*}$ (right column) in expanded four-roll mill, for $R e_{e n t}=5, \lambda=1, y_{o}{ }^{*}=0$, and distinct microelements.

The time evolution of the deformation of the vector aligned in the $a_{1}$ direction is plotted in Figure 4.18 for the respective combinations of $G$ and $\alpha$. An arbitrary critical deformation is defined as $R_{c r i t}^{*}=10$. Consequently, the drop will break if $R^{*} \geq 10$ or $R^{*} \leq 0.01$. A few other values are indicated with black dots on the Figures in order to accommodate future drop breakup criteria, for illustration purposes. They are $R_{c r i t}^{*}=3,5$, and 7 . It means that the drop will break if $R^{*} \geq 3$ or $R^{*} \leq \frac{1}{9}, R^{*} \geq 5$ or $R^{*} \leq \frac{1}{25}$, and $R^{*} \geq 7$ or $R^{*} \leq \frac{1}{49}$, respectively. 


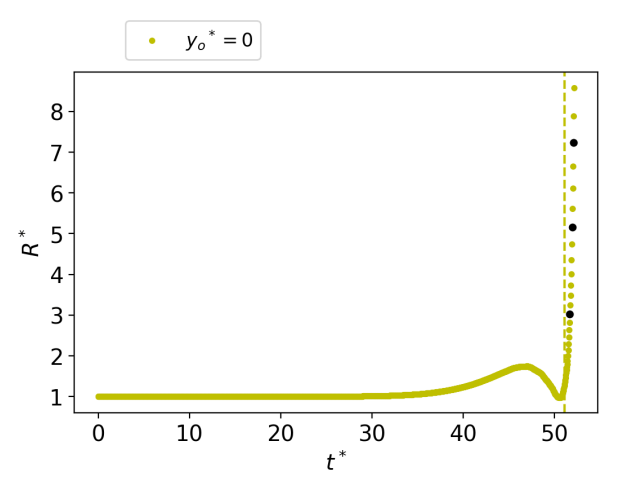

i) $G=2 ; \alpha=0$

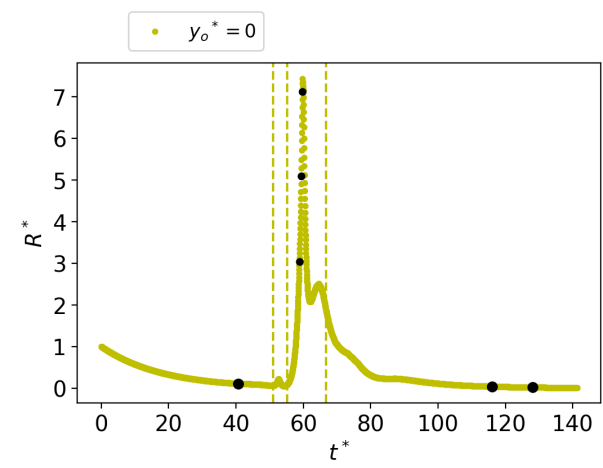

iii) $G=1 ; \alpha=0.2$

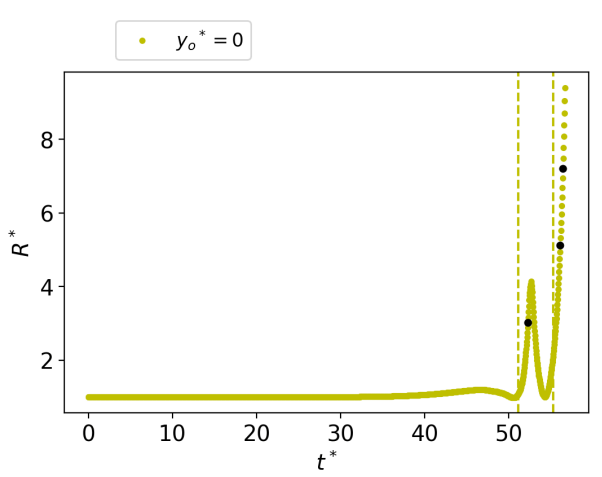

ii) $G=1 ; \alpha=0$

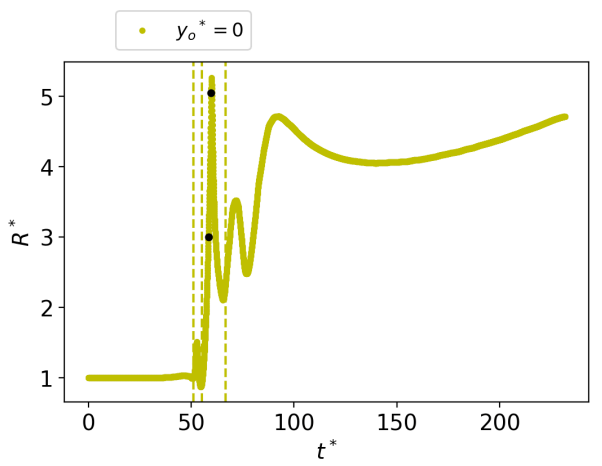

iv) $G=0.4 ; \alpha=0$

Figure 4.18: $R^{*}$ versus $t^{*}$ in the expanded four-roll mill with $R e_{\text {ent }}=5, \lambda=1$, $y_{0}{ }^{*}=0$, and distinct microelements.

For the pair of $G=2$ and $\alpha=0$ represented in the figure $4.18 \mathrm{i}$ ), the microelement experiences a huge and sudden extension right after reaching the inlet of the internal region of the four-roll mill. As already mentioned, the nondimensional time pertaining to the internal region inlet is $t^{*}=51.12$. The drop breakage times are respectively $t^{*}$ breakage $=51.70,51.95,52.09$, and 52.16 for the critical deformations associated with $R^{*}{ }_{\text {crit }}=3,5,7$, and 10 . These results are justified by the fact that the microelement goes through a region of almost pure extension near the center of the four-mill, which can be seen in Figure $4.16 \mathrm{i}$ ). Recalling the application of this work, this is an unfavorable scenario due to the fact that the breakup results in smaller drops, which reduces the probability of droplets coalescing and favors the stability of the emulsion.

In the case shown in Figure 4.18 ii) $(G=1$ and $\alpha=0)$, it happens the same, from the emulsion point of view. In the case displayed in the figure 4.18 iii), i.e., $G=1$ and $\alpha=0.2$, the drop initially experiences compression associated with the region of almost pure extension near the "West" boundary that might be visualized in Figure 4.16 iii). In the sequence, 
the drop experiences no major deformation for a period of time before reaching the internal region of the four-roll mill. Then, it suddenly starts to elongate mainly because the combination of angular velocities of the rollers pulls the microelement both upwards and downwards in this region. As the microelement is moved to the upper region of the four-roll mill, it starts to experience a combination of shear and compression. After leaving the four-roll mill, the drop faces another region of mainly pure extension, which is associated with compression. Then, the microelement vector length keeps decreasing until the final breakage. Notice that during this distinct moments, a few dot markers were plotted. First, $R^{*}=\frac{1}{9}$ at time $t^{*}=40.75$ is a indication as possible breakage. In the fast elongation right after the center of the four-roll mill, the markers of $R^{*}=3,5$, and 7 are respectively reached at times $t^{*}=58.84$, $59.38,59.74$. After the four-roll mill and long periods of compression we have the analogous values for compression $R^{*}=\frac{1}{25}$ and $\frac{1}{49}$ at respective times $t^{*}=116.10$ and 128.09 , and finally, according to our chosen criterion $R^{*}=0.01$ at $t^{*}=141.30$, interrupting the code. Thus, in most cases examined, the drop will eventually break up and will favor the stability of the emulsion.

However, the case presented in Figure 4.18 iv), in which $G=0.4$ and $\alpha=0$, the modified persistence-of-straining field indicates predominantly rigid body motions as Figure 4.16 iv) suggests. Consequently, even the extension region near the center is more subtle. As the plot of deformation implies, right after the center, the elongation is enough to breakup the drop if $R^{*}{ }_{\text {crit }}=3$ or 5 . However for $R_{\text {crit }}^{*}=7$ or 10 , the drop suffers deformation but is not enough to break it. Thus, considering the adopted criterion of $R_{\text {crit }}^{*}=10$, it will reach the West border at time $t^{*}=231.70$ with $R^{*}=4.71$. From the physical point of view, we have one of the best scenarios in this case. There will be less drops of the dispersed phase with larger sizes. Therefore, it increases the probability of happening drop coalescence. It therefore favors the emulsion instability which is desired for the application related with phase separation.

Figure 4.19 shows microelements placed at different vertical positions and their respective pathlines, with $\lambda=0$ and $R e_{\text {ent }}=5$. This is the configuration pertaining to simple shear flow. Notice that the increase of $G$, present in figure $4.19 \mathrm{i}$ ), contributes to the presence of regions of almost pure extension (near the cylinders with non-null angular velocities) and others with the combination of extension and shear. Hence, looking for the microelement initially placed at $y_{o}{ }^{*}=-1.57$, its clear that the microelement breaks very close to the inlet of the internal region. The plot 4.20 i) confirms exactly this information. Analyzing the microelement deformation from Figure $4.21 \mathrm{i}$ ), it is safe to assert that the increase of the parameter $G$ brings elongation for the microelement 
immediately before and in the internal region of the four-roll mill. That justifies the increases of the vector length and the following markers: $R^{*}=3,5$, and 7 at times $t^{*}=47.03,51.41,53.10$ and the final breakage according to the criterion $R^{*}{ }_{\text {crit }}=10$ at time $t^{*}=54.32$.

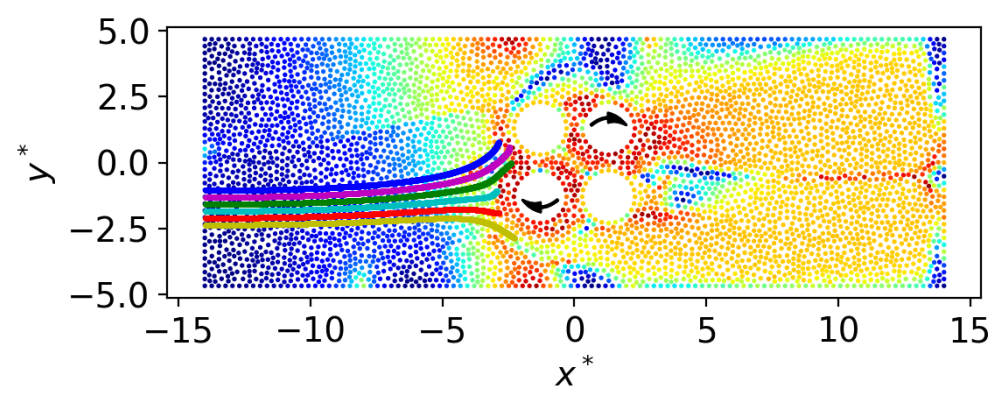

$$
\begin{array}{ll}
\text { - } y_{0}{ }^{*}=-1.05 & \text {. } y_{0}{ }^{*}=-1.84 \\
\text { - } y_{0}{ }^{*}=-1.31 & \text {. } y_{0}{ }^{*}=-2.10 \\
\text { - } y_{0}{ }^{*}=-1.57 & \circ \\
y_{0}{ }^{*}=-2.36
\end{array}
$$
i) $G=2 ; \alpha=0$

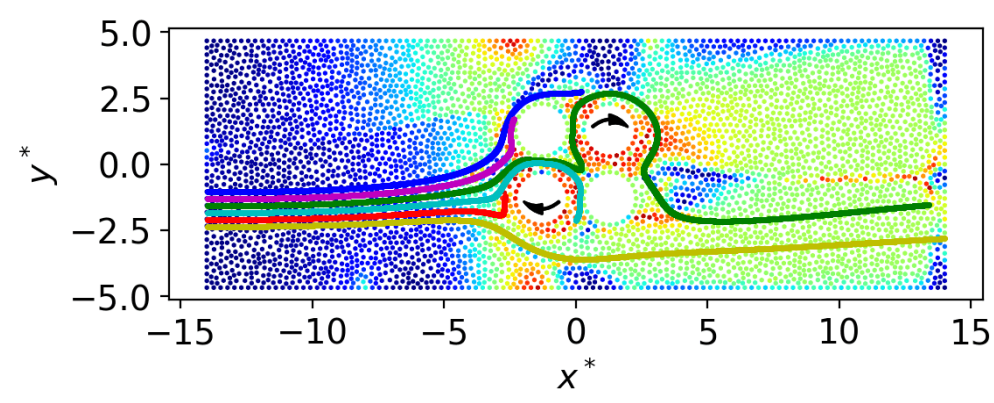

$$
\begin{aligned}
& \text { - } y_{0}{ }^{*}=-1.05 \quad \text {. } y_{0}{ }^{*}=-1.84 \\
& \text { - } y_{0}{ }^{*}=-1.31 \text { - } y_{0}{ }^{*}=-2.10 \\
& \text { - } y_{0}{ }^{*}=-1.57 \text {. } y_{0}{ }^{*}=-2.36
\end{aligned}
$$

ii) $G=1 ; \alpha=0$

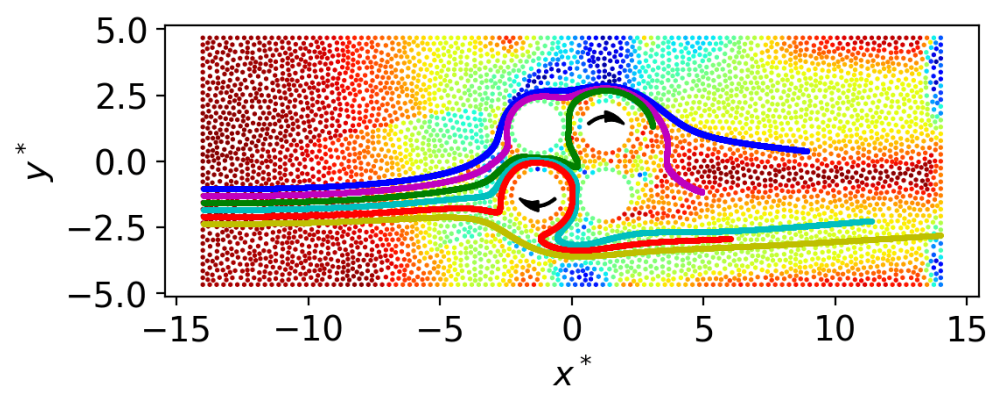

$$
\begin{array}{ll}
\text { - } y_{o}{ }^{*}=-1.05 & \text {. } y_{o}{ }^{*}=-1.84 \\
\text { - } y_{o}{ }^{*}=-1.31 & \text {. } y_{o}{ }^{*}=-2.10 \\
\text { - } y_{o}{ }^{*}=-1.57 & \text {. } y_{o}{ }^{*}=-2.36
\end{array}
$$

iii) $G=1 ; \alpha=0.2$ 


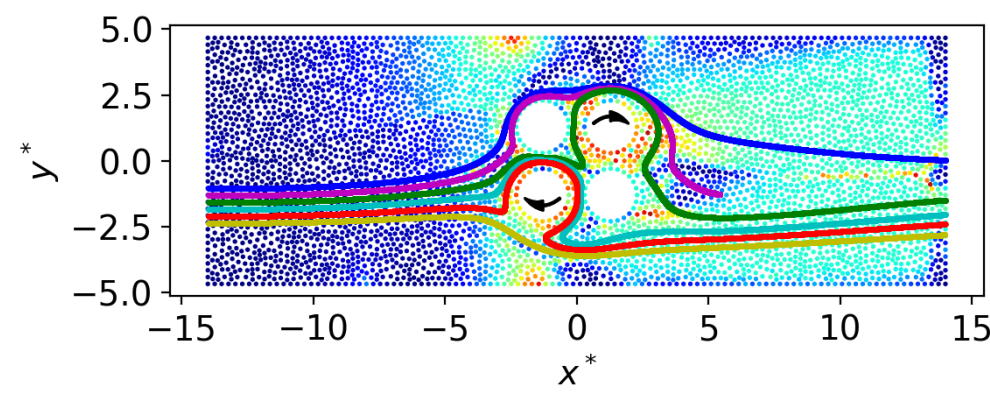

$$
\begin{array}{lll}
\text { - } y_{0}{ }^{*}=-1.05 & \text { - } y_{0}{ }^{*}=-1.84 \\
\text { - } y_{0}{ }^{*}=-1.31 & \text { - } y_{0}{ }^{*}=-2.10 \\
\text { - } y_{0}{ }^{*}=-1.57 & \text { - } y_{0}{ }^{*}=-2.36
\end{array}
$$

iv) $G=0.5 ; \alpha=0$

Figure 4.19: Pathlines in the expanded four-roll mill, for $R e_{e n t}=5, \lambda=0$ and distinct microelements.

Image $4.19 \mathrm{ii})$ shows that the microelement placed at the same original position $y_{o}{ }^{*}=-1.57$ now faces, in its pathline, more regions of combined extension and shear than pure extension in the internal region of the four-roll mill. After leaving the internal region, it deals with a large region of almost simple shear flow, which is responsible for breaking the microelement actually very close to the "East" boundary. Figure 4.20 iii) provides the values of the internal region time markers: $t^{*}=54.78,62.75,80.63$. Once again, according to Figure $4.21 \mathrm{ii}$ ), the microelement is exposed to elongation inside the fourroll mill. The following markers are obtained in this region $R^{*}=3,5$, and 7 at times $t^{*}=67.60,70.44$, and 73.02. Nonetheless, following the criterion of $R_{\text {crit }}^{*}=10$, the microelement only breaks after long periods of shear flow at time $t^{*}=217.91$.

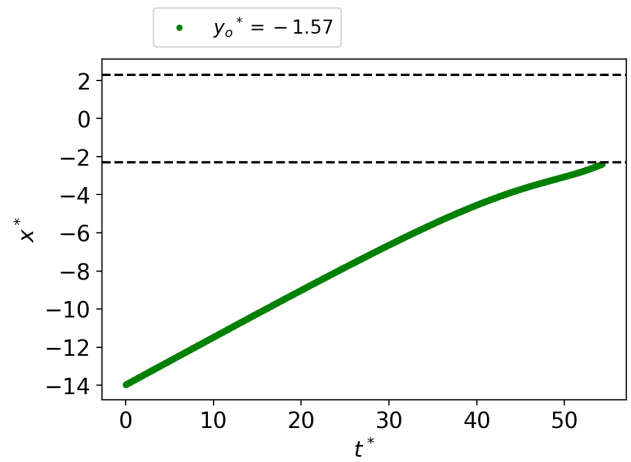

i) $G=2 ; \alpha=0$

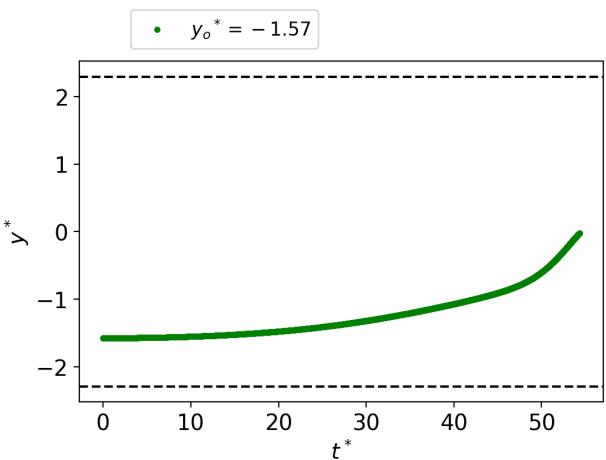

ii) $G=2 ; \alpha=0$ 

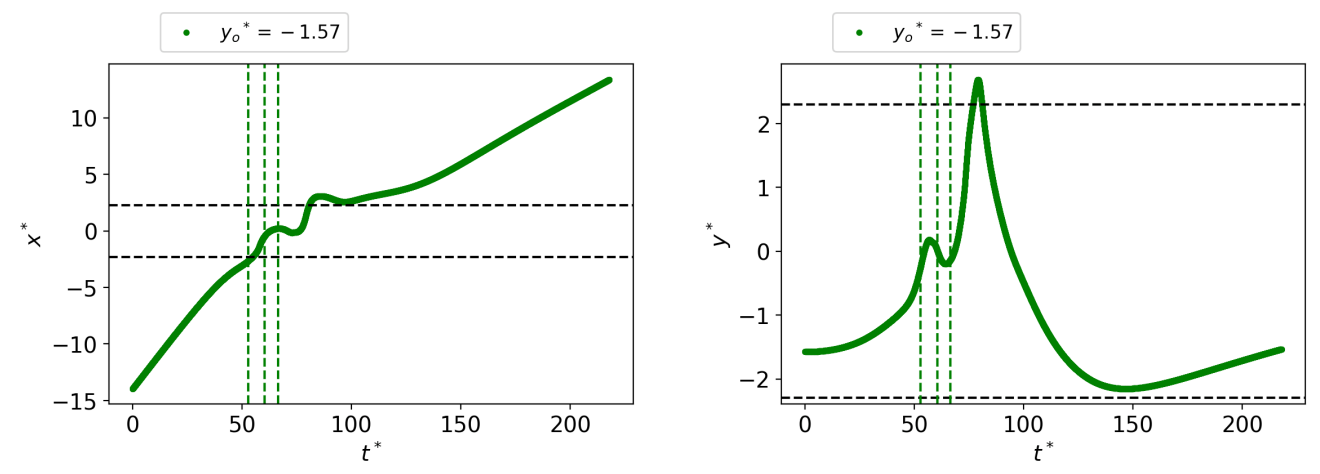

iii) $G=1 ; \alpha=0$

iv) $G=1 ; \alpha=0$
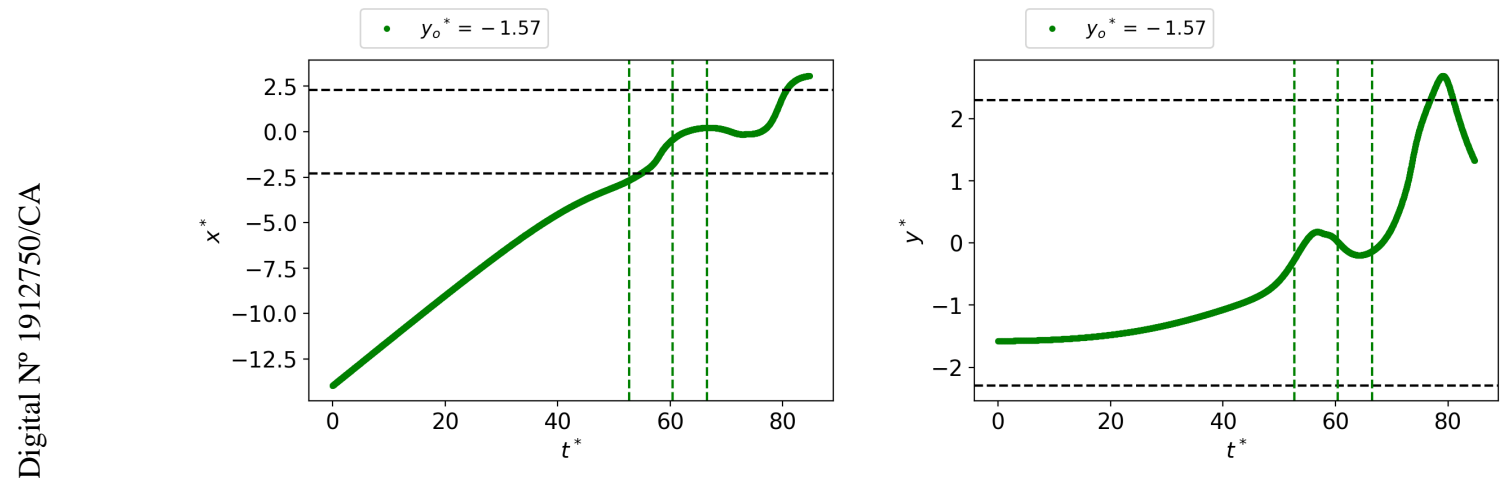

v) $G=1 ; \alpha=0.2$

vi) $G=1 ; \alpha=0.2$
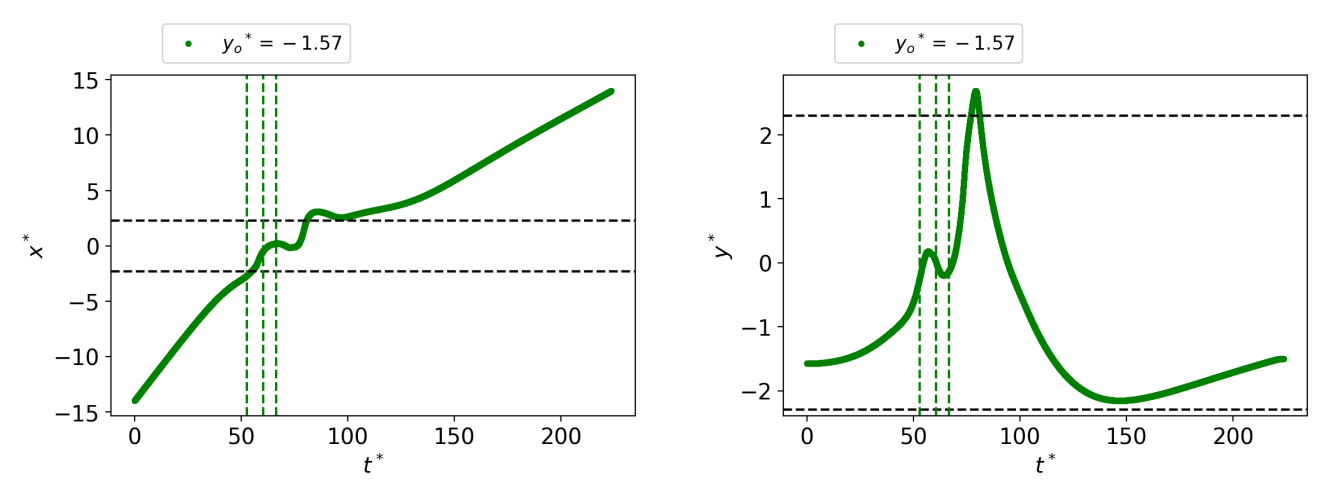

vii) $G=0.5 ; \alpha=0$

viii) $G=2 ; \alpha=0$

Figure 4.20: $x^{*}$ versus $t^{*}$ (left column) and $y^{*}$ versus $t^{*}$ (right column) in the expanded four-roll mill, for $R e_{e n t}=5, \lambda=0, y_{o}{ }^{*}=-1.57$, and distinct microelements. 


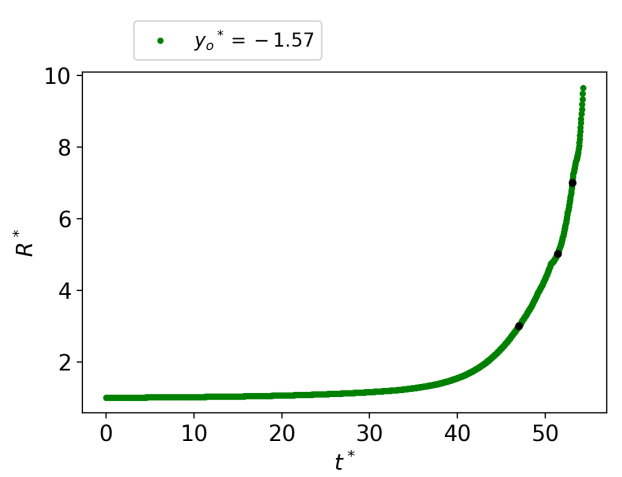

i) $G=2 ; \alpha=0$

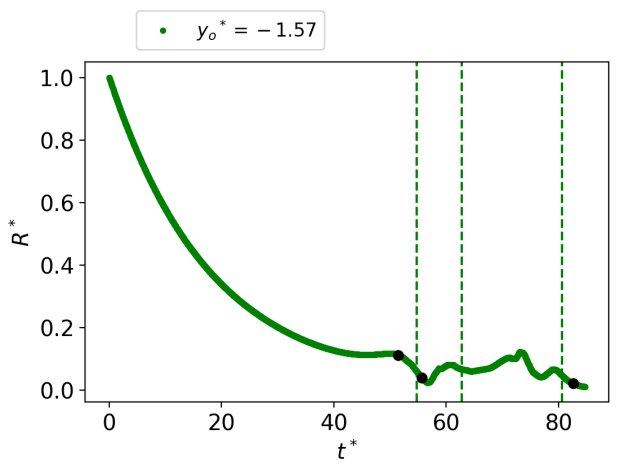

iii) $G=1 ; \alpha=0.2$

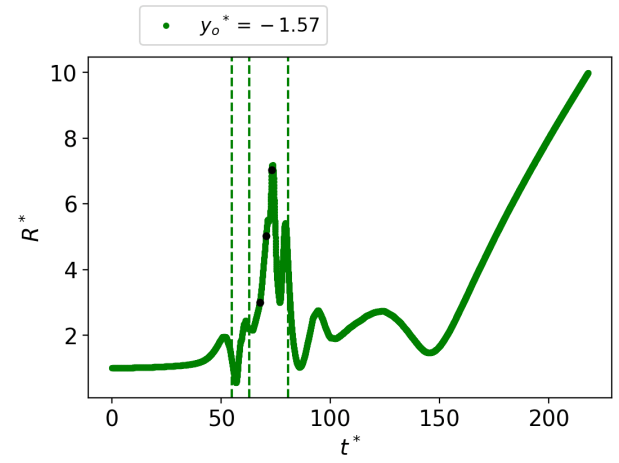

ii) $G=1 ; \alpha=0$

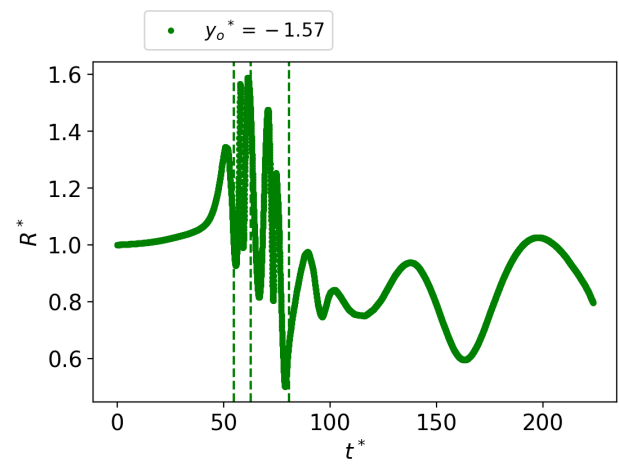

iv) $G=0.5 ; \alpha=0$

Figure 4.21: $R^{*}$ versus $t^{*}$ in the expanded four-roll mill, for $R e_{e n t}=5, \lambda=0$, $y_{o}{ }^{*}=-1.57$, and distinct microelements.

The increase of $\alpha$ also brings more extension as can be seen in Figure 4.19 iii). Not only near the "West" border, but also after the rollers between the range of $-2.5 \leq y^{*} \leq 2.5$. Every single dash marked is reached in Figure 4.20 v) and vi). The microelement leaves the internal region at time $t^{*}=80.63$ and breaks at $t^{*}=84.72$. According to the deformation plot in Figure 4.21 iii), the microelement is compressed since the beginning. Thus the increase of $\alpha$ brings compression to the external region. During this time, the following markers were reached: $R^{*}=\frac{1}{9}, \frac{1}{25}$, and $\frac{1}{49}$ at times $t^{*}=51.41,55.64$, and 82.58 (the first marker right before the internal region, the second in the internal region and the last one right after the internal region). Finally the microstructure breaks with $R^{*}=\frac{1}{100}$ at time $t^{*}=84.72$.

Figure $4.19 \mathrm{iv)}$ illustrates the effect of decreasing the parameter $G$. The modified persistence-of-straining field becomes predominantly rigid body motion before the rollers and a combination of rigid body motion and shear flow after the rollers. In the internal region, near the static cylinders, there is also the combination of rigid body motion and shear flow, while the rotating cylinders 
cause a combination of shear and extensional. Therefore, the deformations are smaller in this case. Notice from Figure $4.21 \mathrm{iv)}$ that the microelement is exposed to a lot of shear in many directions, which leads to a sequence of increase and decrease of the respective length vector. The microelement does not break and reaches the "East" border at time $t^{*}=223.66$ with $R^{*}=0.80$.

From the emulsion point of view, a few scenarios are repeated. For the cases with the pairs $G=2$ and $\alpha=0, G=1$ and $\alpha=0$, the drop breaks up by elongation. Notice that for the microelement with $G=2$ and $\alpha=0$ it was exposed to a extension region, while for $G=1$ and $\alpha=0$ it was exposed to a shear region and both of them were able to provide enough elongation. For the case with $G=1$ and $\alpha=0.2$, the drop reaches the critical value of compression and consequently leads to elongation in the other orthogonal directions $\left(a_{2}\right.$ and $\left.a_{3}\right)$. In both of these conditions, it increases the number of drops and decreases their respective sizes. Thus, it reduces the probability of coalescence between drops and results in a more stable emulsion. In contrast, in the case of $G=0.5$ and $\alpha=0$, the microelement does not break up. Since it reaches the "East" boundary with $R^{*}=0.80$, it represents a elongation of the drop of $11.80 \%$ in the direction of axis $a_{2}$ and $a_{3}$. It leads to the decrease of the number of drops and increases their respective sizes. It is the desired case for the application since favors the probability of coalescence. 


\section{5}

\section{Final Remarks}

In this work, simulations were implemented to analyze the mechanical behavior of emulsions in the four-roll mill. The main benefit of using the fourroll mill is the possibility of obtaining several flow configurations depending on the choice of the imposed angular velocity of the rollers.

Firstly, the approach of classifying the flows of the four-roll mill is discussed. Therefore, the case of an incompressible, bi-dimensional and isothermal flow of a Newtonian fluid in the four-roll mill at steady-state, representing the continuous phase, was initially implemented. The results obtained of velocity fields and streamlines were compatible with the literature. The flow classification criteria proposed by Thompson and de Souza Mendes [36] was used to investigate if the flow configuration parameter $\lambda$ was suitable with the flow classifications established in the literature such as pure extensional flow, pure shear flow and rigid body movement respectively associated with the flow-type parameter $\lambda=1, \lambda=0$, and $\lambda=-1$. The obtained results demonstrate that, for several configurations, there is a local classification for each position and, most of the time, the fluid experiences more than one mechanical behavior. Therefore, it is inaccurate to use a general flow classification for a single flow configuration.

Secondly, a post-processing was developed to consider the flow classification of the dispersed phase. Despite of the velocity field being the same, microelements of the dispersed phased, represented by passive vectors, display completely distinct flow classification from the continuous phase at the same position. Therefore, it only reinforces that it is not appropriate to use a general classification. Each position will have its own flow classification depending on the time and whether it is currently being occupied by which phase.

The deformation of these microelements were also examined. Employing the work of Olbricht [43], the vectors were aligned to one of the axis from the ellipsoid that represents the small droplet of the dispersed phase. Considering the volume conservation, the deformation of the vector might lead to breakage. A criterion based on maximum deformation of any of the three axis was defined. Both successful and unsuccessful scenarios were gathered in terms of the phase separation application. The successful case happens when the microelements 
deforms considerably without breaking the drop. Thus, it results in fewer drops with larger sizes in the domain. It increases the probability of coalescence and phase separation.

Therefore, this work does not provide ready to apply results. However, it brings relevant qualitative insights about distinct flows and the deformation of droplets from the dispersed phase of the emulsions in the four-roll mill.

\section{1}

\section{Future Works}

It is important to adjust and consider a few topics for future works. An important issue in this work is the fact that just a few microelements that are presented in the inlet border reach the internal region of the four-roll mill. A possible solution is to insert an inclined barrier before the rollers to redirect the particles to the internal region.

Besides, a great upgrade would be to consider the droplet as a secondorder tensor instead of a vector. Thus, deformations in other directions according to volume conservation would be more accurate. This would also contribute to specifying the emulsions phases. Then, it would be possible to simulate applications cases of water-in-oil emulsions (W/O).

At last, an important and difficult task is to establish an criterion for the drop breakup. This was not considered in this work and instead the drop breakup was based exclusively on arbitrary values of maximum deformation. 


\section{Bibliography}

\section{References}

[1] LOPETINSKY, R. J. G.; MASLIYAH, J. H.; XU, Z.. Colloidal Particles at Liquid Interfaces. Cambridge University Press, 2006.

[2] HIGDON, J. J. L.. The kinematics of the four-roll mill. Physics of Fluids A: Fluid Dynamics, 5(1):274-276, jan 1993.

[3] LAGNADO, R. R.; LEAL, L. G.. Visualization of three-dimensional flow in a four-roll mill. Experiments in Fluids, 9(1-2):25-32, 1990.

[4] LANGeVin, D.; POTEAU, S.; HÉnAut, I. ; ARGILlier, J. F.. Crude oil emulsion properties and their application to heavy oil transportation. Oil \& Gas Science and Technology, 59(5):511-521, sep 2004.

[5] BORTOLOTI, G. B.; VIGANÔ, G. C.; ZUCATELLI, P. J. ; ROMERO, O. J.. Injeção de emulsões e seu impacto na produção de petróleo. Latin American Journal of Energy Research, 2(1):8-14, aug 2015.

[6] SPIECKER, P. M.; KILPATRICK, P. K.. Interfacial rheology of petroleum asphaltenes at the oil-water interface. Langmuir, 20(10):4022-4032, 2004. PMID: 15969394.

[7] LAKE, L.; ARNOLD, K.; FANCHI, J. ; OF PETROLEUM ENGINEERS (U.S.), S.. Petroleum Engineering Handbook. Número v. 3 em Petroleum Engineering Handbook. Society Of Petroleum Engineers, 2006.

[8] FORTUNY, M.; OliVEIRA, C. B. Z.; MELO, R. L. F. V.; NELE, M.; COUTINHO, R. C. C. ; SANTOS, A. F.. Effect of salinity, temperature, water content, and $\mathrm{pH}$ on the microwave demulsification of crude oil emulsions. Energy \& Fuels, 21(3):1358-1364, 2007.

[9] FONSECA, M. B.; PEREIRA, M. L.; JUSTINIANO, M. R. ; SANTANA, R. C.. Geração de emulsões de petróleo A/O e O/A sem a adição de surfactante. Latin American Journal of Energy Research, 3(1):10-16, sep 2016.

[10] KABALNOV, A.. Thermodynamic and theoretical aspects of emulsions and their stability. Current Opinion in Colloid \& Interface Science, 3(3):270 - 275, 1998. 
[11] KILPATRICK, P.. Water-in-crude oil emulsion stabilization: Review and unanswered questions. Energy \& Fuels, 26:4017-4026, 062012.

[12] TADROS, T. F.. Emulsion Science and Technology: A General Introduction, chapter 1, p. 1-56. John Wiley \& Sons, Ltd, 2009.

[13] The formation of emulsions in definable fields of flow. Proceedings of the Royal Society of London. Series A, Containing Papers of a Mathematical and Physical Character, 146(858):501-523, oct 1934.

[14] RUMSCHEIDT, F.; MASON, S.. Particle motions in sheared suspensions XII. Deformation and burst of fluid drops in shear and hyperbolic flow. Journal of Colloid Science, 16(3):238-261, jun 1961.

[15] GIESEKUS, H.. Strömungen mit konstantem geschwindigkeitsgradienten und die bewegung von darin suspendierten teilchen. Rheologica Acta, 2(2):112-122, jun 1962.

[16] COX, R. G.. The deformation of a drop in a general timedependent fluid flow. Journal of Fluid Mechanics, 37(3):601-623, jul 1969.

[17] FULLER, G. G.; LEAL, L. G.. Flow birefringence of dilute polymer solutions in two-dimensional flows. Rheologica Acta, 19(5):580-600, Sept. 1980.

[18] TORZA, S.. Shear-induced crystallization of polymers. i. the fourroller apparatus. Journal of Polymer Science: Polymer Physics Edition, 13(1):43-57, Jan. 1975.

[19] MCHUGH, A. J.; GUY, R. K. ; TREE, D. A.. Extensional flow-induced crystallization of a polyethylene melt. Colloid \& Polymer Science, 271(7):629-645, jul 1993.

[20] BENTLEY, B. J.; LEAL, L. G.. A computer-controlled four-roll mill for investigations of particle and drop dynamics in twodimensional linear shear flows. Journal of Fluid Mechanics, 167(-1):219, jun 1986.

[21] BENTLEY, B. J.; LEAL, L. G.. An experimental investigation of drop deformation and breakup in steady, two-dimensional linear flows. Journal of Fluid Mechanics, 167(-1):241, jun 1986. 
[22] GRACE, H. P.. Dispersion phenomena in high viscosity immiscible fluid systems and application of static mixers as dispersion devices in such systems. Chemical Engineering Communications, 14(36):225-277, mar 1982.

[23] SHERWOOD, J. D.. Tip streaming from slender drops in a nonlinear extensional flow. Journal of Fluid Mechanics, 144:281-295, jul 1984.

[24] FENG, J.; LEAL, L.. Numerical simulations of the flow of dilute polymer solutions in a four-roll mill. Journal of Non-Newtonian Fluid Mechanics, 72(2-3):187-218, oct 1997.

[25] INNINGS, F.; HAMBERG, L. ; TRÄGÅRDH, C.. Dynamic modelling of the deformation of a drop in a four-roll mill. Chemical Engineering Science, 60(17):4771-4779, sep 2005.

[26] YANG, H.; PARK, C. C.; HU, Y. T. ; LEAL, L. G.. The coalescence of two equal-sized drops in a two-dimensional linear flow. Physics of Fluids, 13(5):1087-1106, may 2001.

[27] ASTARITA, G.. Objective and generally applicable criteria for flow classification. Journal of Non-Newtonian Fluid Mechanics, 6(1):69-76, jan 1979.

[28] ASTARITA, G.. Two dimensionless groups relevant in analysis of steady flows of viscoelastic materials. Industrial \& Engineering Chemistry Fundamentals, 6(2):257-262, may 1967.

[29] HUILGOL, R. R.. On the concept of the Deborah number. Transactions of the Society of Rheology, 19(2):297-306, jul 1975.

[30] ASTARITA, G., . M. G.. Principles of non-Newtonian fluid mechanics. London: McGraw-Hill, 1974.

[31] TANNER, R. I.; HUILGOL, R. R.. On a classification scheme for flow fields. Rheologica Acta, 14(11):959-962, nov 1975.

[32] TANNER, R. I.. A test particle approach to flow classification for viscoelastic fluids. AIChE Journal, 22(5):910-918, sep 1976.

[33] DENN, M. M.. The Mechanics of Viscoelastic Flow, volume 22, pp 101-124. A. S. M. E, AMD, 1977. 
[34] DROUOT, R.; LUCIUS, R.. Approximation du second ordre de la loi de comportement des fluides simples. lois classiques deduites de l'introduction d'un noveau tenseur objectif. ARCH. MED, Poland, Da. 1976, Vol 28, no 2, pp. 189-198, abs. pol. russe, bibl. 14 ref., 1976.

[35] HUILGOL, R.. Comments on "objective and generally applicable criteria for flow classification", by g. astarita. Journal of NonNewtonian Fluid Mechanics, 7(1):91-95, jan 1980.

[36] THOMPSON, R. L.; SOUZA MENDES, P. R.. Persistence of straining and flow classification. International Journal of Engineering Science, 43(1):79 - 105, 2005.

[37] BERCOVIER, M.; PIRONNEAU, O.. Error estimates for finite element method solution of the stokes problem in the primitive variables. Numerische Mathematik, 33(2):211-224, jun 1979.

[38] LEE, Y.-J.; LI, H.. On stability, accuracy, and fast solvers for finite element approximations of the axisymmetric stokes problem by hood-taylor elements. SIAM Journal on Numerical Analysis, 49(1/2):668-691, 2011.

[39] GUZMÁN, J.; SÁNCHEZ, M. A.. Max-norm stability of low order taylor-hood elements in three dimensions. Journal of Scientific Computing, 65(2):598-621, jan 2015.

[40] PAPAdOPOUlOS, P.; TAYLOR, R. L.. A generalized Newton method for higher-order finite element approximations in non-linear elasticity. International Journal for Numerical Methods in Engineering, 39(15):2635-2646, aug 1996.

[41] SHADID, J. N.. A fully-coupled newton-krylov solution method for parallel unstructured finite element fluid flow, heat and mass transfer simulations. International Journal of Computational Fluid Dynamics, 12(3-4):199-211, jan 1999.

[42] KIM, S. D.; LEE, Y. H. ; SHIN, B. C.. Newton's method for the navier-stokes equations with finite-element initial guess of stokes equations. Computers \& Mathematics with Applications, 51(5):805-816, mar 2006.

[43] OLBRICHT, W.; RALLISON, J. ; LEAL, L.. Strong flow criteria based on microstructure deformation. Journal of Non-Newtonian Fluid Mechanics, 10(3-4):291-318, jan 1982. 
[44] NACCACHE, J. P. A.. Desenvolvimento de emulsões modelo para mimetizar propriedades físicas de emulsões de petróleo. Master's thesis, 2020. 


\section{Codes}

Every code used in this work is available in this section. It includes the meshes and the main simulation.

\section{A.1 \\ Meshes}

Both meshes were implement with the software Gmsh. First, 4RM_Mesh_Classic.geo, which is associated with the classic four-roll mill, is presented. Then, the code associated with the expanded four-roll mill named 4RM_Mesh_Expanded.geo is shown.

Listing A.1: 4RM_Mesh_Classic.geo

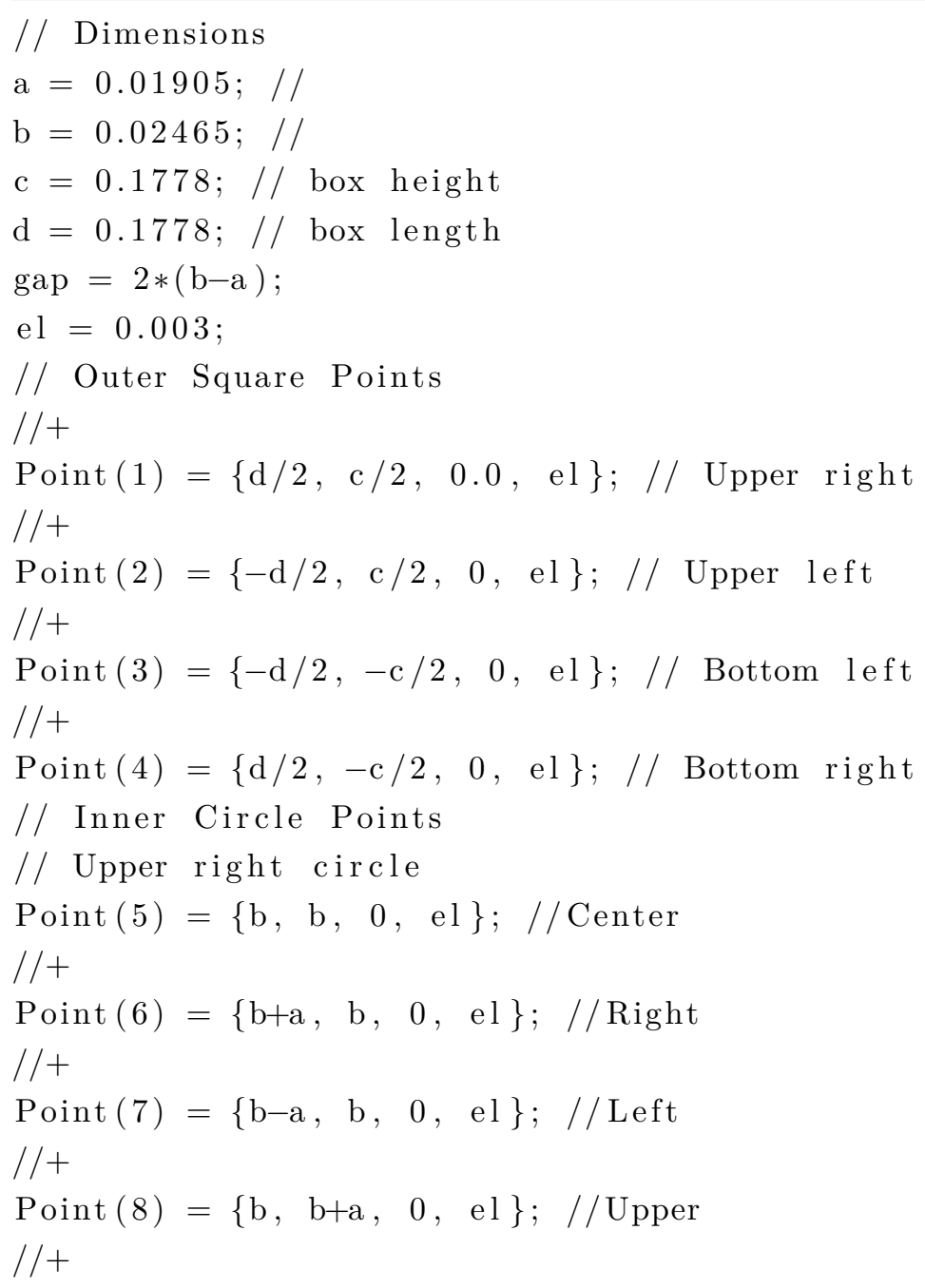


Point $(9)=\{\mathrm{b}, \mathrm{b}-\mathrm{a}, 0$, el $\} ; / /$ Lower

// Upper left circle

Point $(10)=\{-\mathrm{b}, \mathrm{b}, 0, \mathrm{el}\} ; / /$ Center

$/ /+$

Point $(11)=\{-\mathrm{b}+\mathrm{a}, \mathrm{b}, 0, \mathrm{el}\} ; / /$ Right

//+

Point $(12)=\{-\mathrm{b}-\mathrm{a}, \mathrm{b}, 0, \mathrm{el}\} ; / /$ Left

$/ /+$

Point $(13)=\{-\mathrm{b}, \mathrm{b}+\mathrm{a}, 0, \mathrm{el}\} ; / /$ Upper

//+

Point $(14)=\{-\mathrm{b}, \mathrm{b}-\mathrm{a}, 0, \mathrm{el}\} ; / /$ Lower

// Bottom left circle

Point $(15)=\{-\mathrm{b},-\mathrm{b}, 0, \mathrm{el}\} ; / /$ Center

//+

Point $(16)=\{-\mathrm{b}+\mathrm{a},-\mathrm{b}, 0$, el $\} ; / /$ Right

$/ /+$

Point $(17)=\{-\mathrm{b}-\mathrm{a},-\mathrm{b}, 0, \mathrm{el}\} ; / /$ Left

//+

Point $(18)=\{-\mathrm{b},-\mathrm{b}+\mathrm{a}, 0, \mathrm{el}\} ; / /$ Upper

$/ /+$

Point $(19)=\{-\mathrm{b},-\mathrm{b}-\mathrm{a}, 0, \mathrm{el}\} ; / /$ Lower

// Bottom right circle

Point $(20)=\{b,-b, 0$, el $\} ; / /$ Center

$/ /+$

Point $(21)=\{\mathrm{b}-\mathrm{a},-\mathrm{b}, 0$, el $\} ; / /$ right

$/ /+$

Point $(22)=\{\mathrm{b}+\mathrm{a},-\mathrm{b}, 0, \mathrm{el}\} ; / /$ left

$/ /+$

Point $(23)=\{\mathrm{b},-\mathrm{b}+\mathrm{a}, 0$, el $\} ; / /$ Upper

$/ /+$

Point $(24)=\{\mathrm{b},-\mathrm{b}-\mathrm{a}, 0$, el $\} ; / /$ Lower

// Rectangle Lines

$/ /+$

Line $(25)=\{1,2\} ; / /$ North

$/ /+$

Line $(26)=\{2,3\} ; / /$ West

$/ /+$

Line $(27)=\{3,4\} ; / /$ South

$/ /+$

Line $(28)=\{4,1\} ; / /$ East

// Circle Lines

// Upper right circle

// Northeast UR

Circle $(29)=\{6,5,8\}$;

// Northwest UR

Circle $(30)=\{8,5,7\}$;

// Southwest UR 
Circle $(31)=\{7,5,9\}$;

// Southeast UR

Circle $(32)=\{9,5,6\}$;

// Upper left circle

// Northeast UL

Circle $(33)=\{11,10,13\}$;

// Northwest UL

Circle $(34)=\{13,10,12\}$;

// Southwest UL

Circle $(35)=\{12,10,14\}$;

// Southeast UL

Circle $(36)=\{14,10,11\}$;

// Bottom left circle

// Northeast BL

Circle $(37)=\{16,15,18\}$;

// Northwest BL

Circle $(38)=\{18,15,17\}$;

// Southwest BL

Circle $(39)=\{17,15,19\}$;

// Southeast BL

Circle $(40)=\{19,15,16\}$;

// Bottom right circle

// Northeast BR

Circle $(41)=\{21,20,23\}$;

// Northwest BR

Circle $(42)=\{23,20,22\}$;

// Southwest BR

Circle $(43)=\{22,20,24\}$;

// Southeast BR

Circle $(44)=\{24,20,21\}$;

// Line Loops

$/ /+$

Line $\operatorname{Loop}(45)=\{25,26,27,28\}$;

$/ /+$

Line $\operatorname{Loop}(46)=\{29,30,31,32\}$;

$/ /+$

Line $\operatorname{Loop}(47)=\{33,34,35,36\}$;

$/ /+$

Line $\operatorname{Loop}(48)=\{37,38,39,40\}$;

$/ /+$

Line $\operatorname{Loop}(49)=\{41,42,43,44\}$;

// Surfaces

$/ /+$

Plane Surface $(50)=\{45,46,47,48,49\}$;

// Subdomains

$/ /+$

Physical Line("NorthLine" $)=\{25\}$; 
$/ /+$

Physical Line("WestLine" $)=\{26\}$;

$/ /+$

Physical Line("SouthLine" $)=\{27\}$;

$/ /+$

Physical Line("EastLine" $)=\{28\}$;

$/ /+$

Physical Line("UP_Obstacle_NE" $)=\{29\}$;

$/ /+$

Physical Line("UP_Obstacle_NW" $)=\{30\}$;

$/ /+$

Physical Line("UP_Obstacle_SW" $)=\{31\}$;

$/ /+$

Physical Line("UP_Obstacle_SE" $)=\{32\}$;

$/ /+$

Physical Line("UL_Obstacle_NE" $)=\{33\}$;

$/ /+$

Physical Line("UL_Obstacle_NW") $=\{34\}$;

//

Physical Line("UL_Obstacle_SW" $)=\{35\}$;

$/ /+$

Physical Line("UL_Obstacle_SE") $=\{36\}$;

//+

Physical Line("BL_Obstacle_NE" $)=\{37\}$;

$/ /+$

Physical Line("BL_Obstacle_NW" $)=\{38\}$;

//

Physical Line("BL_Obstacle_SW" $)=\{39\}$;

$/ /+$

Physical Line("BL_Obstacle_SE" $)=\{40\}$;

$/ /+$

Physical Line("BR_Obstacle_NE" $)=\{41\}$;

$/ /+$

Physical Line("BR_Obstacle_NW" $)=\{42\}$;

//

Physical Line("BR_Obstacle_SW" $)=\{43\}$;

$/ /+$

Physical Line("BR_Obstacle_SE" $)=\{44\}$;

$/ /+$

Physical Surface("Fluid") $=\{50\}$; 
Listing A.2: 4RM_Mesh_Expanded.geo

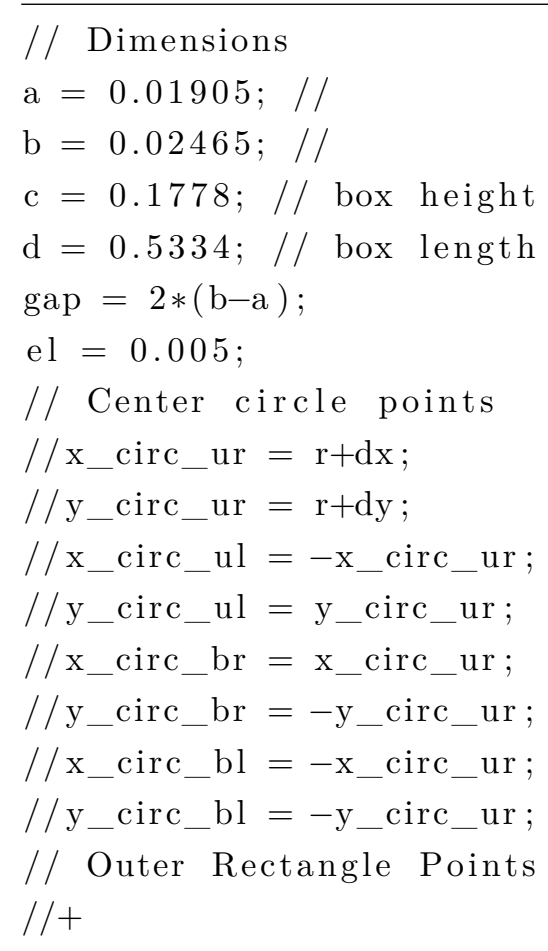

Point $(1)=\{\mathrm{d} / 2, \mathrm{c} / 2,0.0, \mathrm{el}\} ; / /$ Upper right

//+

Point $(2)=\{-\mathrm{d} / 2, \mathrm{c} / 2,0, \mathrm{el}\} ; / /$ Upper left

//+

Point $(3)=\{-\mathrm{d} / 2,-\mathrm{c} / 2,0$, el $\} ; / /$ Bottom left

$/ /+$

Point $(4)=\{\mathrm{d} / 2,-\mathrm{c} / 2,0, \mathrm{el}\} ; / /$ Bottom right

// Inner Circle Points

// Upper right circle

Point $(5)=\{\mathrm{b}, \mathrm{b}, 0, \mathrm{el}\} ; / /$ Center

//+

Point $(6)=\{\mathrm{b}+\mathrm{a}, \mathrm{b}, 0, \mathrm{el}\} ; / /$ Right

$/ /+$

Point $(7)=\{\mathrm{b}-\mathrm{a}, \mathrm{b}, 0, \mathrm{el}\} ; / /$ Left

$/ /+$

Point $(8)=\{\mathrm{b}, \mathrm{b}+\mathrm{a}, 0, \mathrm{el}\} ; / /$ Upper

$/ /+$

Point $(9)=\{\mathrm{b}, \mathrm{b}-\mathrm{a}, 0$, el $\} ; / /$ Lower

// Upper left circle

Point $(10)=\{-\mathrm{b}, \mathrm{b}, 0, \mathrm{el}\} ; / /$ Center

$/ /+$

Point $(11)=\{-\mathrm{b}+\mathrm{a}, \mathrm{b}, 0, \mathrm{el}\} ; / /$ Right

$/ /+$

Point $(12)=\{-\mathrm{b}-\mathrm{a}, \mathrm{b}, 0, \mathrm{el}\} ; / /$ Left

$/ /+$

Point $(13)=\{-\mathrm{b}, \mathrm{b}+\mathrm{a}, 0$, el $\} ; / /$ Upper

$/ /+$

Point $(14)=\{-\mathrm{b}, \mathrm{b}-\mathrm{a}, 0$, el $\} ; / /$ Lower 
// Bottom left circle

Point $(15)=\{-\mathrm{b},-\mathrm{b}, 0, \mathrm{el}\} ; / /$ Center

$/ /+$

Point $(16)=\{-\mathrm{b}+\mathrm{a},-\mathrm{b}, 0, \mathrm{el}\} ; / /$ Right

$/ /+$

Point $(17)=\{-\mathrm{b}-\mathrm{a},-\mathrm{b}, 0, \mathrm{el}\} ; / /$ Left

$/ /+$

Point $(18)=\{-\mathrm{b},-\mathrm{b}+\mathrm{a}, 0, \mathrm{el}\} ; / /$ Upper

$/ /+$

Point $(19)=\{-\mathrm{b},-\mathrm{b}-\mathrm{a}, 0, \mathrm{el}\} ; / /$ Lower

// Bottom right circle

Point $(20)=\{\mathrm{b},-\mathrm{b}, 0, \mathrm{el}\} ; / /$ Center

//+

Point $(21)=\{\mathrm{b}-\mathrm{a},-\mathrm{b}, 0$, el $\} ; / /$ right

//+

Point $(22)=\{\mathrm{b}+\mathrm{a},-\mathrm{b}, 0, \mathrm{el}\} ; / /$ left

//+

Point $(23)=\{\mathrm{b},-\mathrm{b}+\mathrm{a}, 0$, el $\} ; / /$ Upper

$/ /+$

Point $(24)=\{\mathrm{b},-\mathrm{b}-\mathrm{a}, 0, \mathrm{el}\} ; / /$ Lower

// Rectangle Lines

$/ /+$

Line $(25)=\{1,2\} ; / /$ North

$/ /+$

Line $(26)=\{2,3\} ; / /$ West

$/ /+$

Line $(27)=\{3,4\} ; / /$ South

$/ /+$

Line $(28)=\{4,1\} ; / /$ East

// Circle Lines

// Upper right circle

// Northeast UR

Circle $(29)=\{6,5,8\}$;

// Northwest UR

Circle $(30)=\{8,5,7\}$;

// Southwest UR

Circle $(31)=\{7,5,9\}$;

// Southeast UR

Circle $(32)=\{9,5,6\}$;

// Upper left circle

// Northeast UL

Circle $(33)=\{11,10,13\}$;

// Northwest UL

Circle $(34)=\{13,10,12\}$;

// Southwest UL

Circle $(35)=\{12,10,14\}$;

// Southeast UL 
Circle $(36)=\{14,10,11\}$;

// Bottom left circle

// Northeast BL

Circle $(37)=\{16,15,18\}$;

// Northwest BL

Circle $(38)=\{18,15,17\}$;

// Southwest BL

Circle $(39)=\{17,15,19\}$;

// Southeast BL

Circle $(40)=\{19,15,16\}$;

// Bottom right circle

// Northeast BR

Circle $(41)=\{21,20,23\}$;

// Northwest BR

Circle $(42)=\{23,20,22\}$;

// Southwest BR

Circle $(43)=\{22,20,24\}$;

// Southeast BR

Circle $(44)=\{24,20,21\}$;

// Line Loops

$/ /+$

Line Loop $(45)=\{25,26,27,28\}$;

$/ /+$

Line $\operatorname{Loop}(46)=\{29,30,31,32\}$;

$/ /+$

Line $\operatorname{Loop}(47)=\{33,34,35,36\}$;

$/ /+$

Line $\operatorname{Loop}(48)=\{37,38,39,40\}$;

$/ /+$

Line $\operatorname{Loop}(49)=\{41,42,43,44\}$;

// Surfaces

$/ /+$

Plane Surface $(50)=\{45,46,47,48,49\}$;

// Subdomains

$/ /+$

Physical Line("NorthLine" $)=\{25\}$;

$/ /+$

Physical Line("WestLine" $)=\{26\}$;

//+

Physical Line("SouthLine" $)=\{27\}$;

$/ /+$

Physical Line("EastLine" $)=\{28\}$;

//+

Physical Line("UP_Obstacle_NE" $)=\{29\}$;

//+

Physical Line("UP_Obstacle_NW" $)=\{30\}$;

$/ /+$ 
Physical Line("UP_Obstacle_SW" $)=\{31\}$;

$/ /+$

Physical Line("UP_Obstacle_SE") $=\{32\}$;

//+

Physical Line("UL_Obstacle_NE") $=\{33\}$;

$/ /+$

Physical Line("UL_Obstacle_NW" $)=\{34\}$;

//

Physical Line("UL_Obstacle_SW" $)=\{35\}$;

$/ /+$

Physical Line("UL_Obstacle_SE" $)=\{36\}$;

$/ /+$

Physical Line("BL_Obstacle_NE" $)=\{37\}$;

$/ /+$

Physical Line("BL_Obstacle_NW" $)=\{38\}$;

//

Physical Line("BL_Obstacle_SW" $)=\{39\}$;

$/ /+$

Physical Line("BL_Obstacle_SE" $)=\{40\}$;

$/ /+$

Physical Line("BR_Obstacle_NE" $)=\{41\}$;

$/ /+$

Physical Line("BR_Obstacle_NW" $)=\{42\}$;

//

Physical Line("BR_Obstacle_SW" $)=\{43\}$;

$/ /+$

Physical Line("BR_Obstacle_SE" $)=\{44\}$;

$/ /+$

Physical Surface("Fluid" $)=\{50\}$; 


\section{A. 2}

\section{Simulations}

Listing A.3: 4RM.py

\#\%\% Package import

from dolfin import *

from mshr import *

import matplotlib.pyplot as plt

from matplotlib.colors import LogNorm

import matplotlib.cm as $\mathrm{cm}$

import numpy as np

import pandas as pd

import seaborn as sns

import scipy.linalg as la

import scipy.interpolate

import cmath as cmt

from timeit import default_timer as timer

import re

from random import *

import os

import matplotlib.patches as mpatches

from matplotlib.collections import PatchCollection

import sys

start $=\operatorname{timer}()$

\#

\# prettyplotnd.py

\#

\#\% Non-dimensional Pretty Plot Function Definition - Creates Velocity I \#and Pressure Plots of the Results

def prettyplotnd (fig, mesh,t,u,p, dicTitle,R, omega, pnlevels=10,resultspath=', , । $\operatorname{cbar} \mathrm{U}=0, \operatorname{cbar} \mathrm{P}=0)$ :

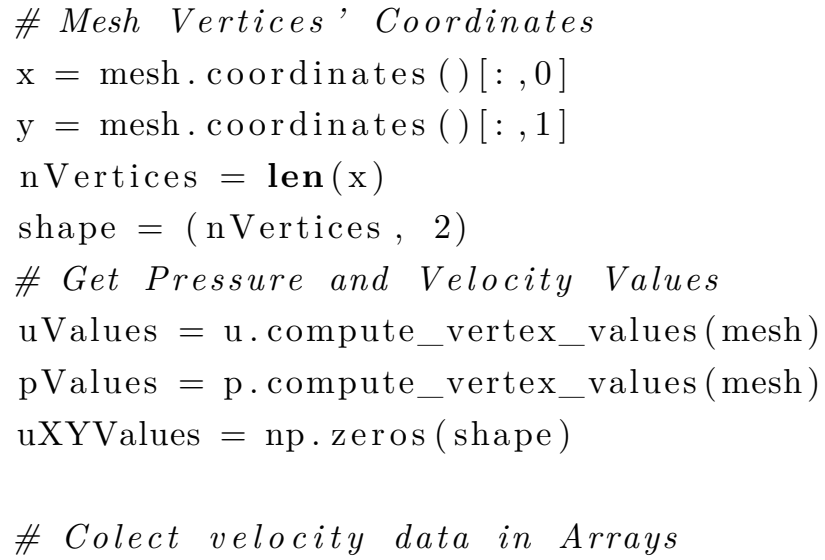


for $\mathrm{j}$ in range $(0, \mathrm{n}$ Vertices $)$ :

$\mathrm{uXYValues}[\mathrm{j}, 0]=\mathrm{uValues}[\mathrm{j}]$

$\mathrm{uXYValues}[\mathrm{j}, 1]=\mathrm{uValues}[\mathrm{j}+\mathrm{nVertices}]$

\section{\# Plot Velocities}

plt.figure $($ num=fig +1, figsize $=(10,10)$, dpi=100, facecolor='w', edgecolor='k')

plt. $\operatorname{clf}()$

$\operatorname{uax}=\operatorname{plot}(\mathrm{u})$

plt.xlabel ( $\mathrm{r}^{\prime} \$ \mathrm{x}{ }^{\prime} \$$, , fontsize $\left.=14\right)$

plt.ylabel $\left(\mathrm{r}^{\prime} \$ \mathrm{y} * \$\right.$, , fontsize $\left.=14\right)$

plt.xticks ( fontsize $=14$ )

plt.yticks ( fontsize $=14$ )

$\mathrm{dpdx}=((\mathrm{pValues}[\mathbf{l e n}(\mathrm{pValues})-1]-\mathrm{pValues}[1]) / \mathrm{x} \cdot \max ())$

\# Calculate Arrow Sizes

$\mathrm{C}=\mathrm{np}$.hypot (uXYValues $[:, 0], \mathrm{uXYValues}[:, 1])$

$\operatorname{minVel}={ }^{\prime}\{: .4 \mathrm{f}\}, \cdot \operatorname{format}(\mathrm{C} \cdot \min () /(\operatorname{abs}(\operatorname{omega} * \mathrm{R})))$

meanVel $=$ ' $\{: .4 \mathrm{f}\}$ ' format $(\mathrm{C} \cdot \operatorname{mean}() /(\operatorname{abs}(\operatorname{omega} * \mathrm{R})))$

$\operatorname{maxVel}=,\{: .4 \mathrm{f}\}, \cdot \operatorname{format}(\mathrm{C} \cdot \max () /(\operatorname{abs}(\operatorname{omega} * \mathrm{R})))$

cbarU $=$ plt. colorbar (uax, orientation=' horizontal')

cbarU.set_ticks ([C.min ()$, C \cdot \operatorname{mean}(), C \cdot \max ()])$

cbarU.ax.set_xticklabels ([minVel, meanVel, maxVel])

cbarU.set_label ( r' $\$ \mathrm{u} * \$$ ', fontsize $=14$, labelpad $=+1)$

cbarU.ax.tick_params ( labelsize=13)

\# Save Figure as. PNG file

if results_path $!=$,' :

plt.savefig (results_path+'velocity_field_lamb='+str (lamb)+

' $\mathrm{Re}=$ '+str (Re_entrance)+'.png', dpi=200)

return cbarU, cbarP, uXYValues, pValues, nVertices;

\section{$\#$}

\# draw_self_loop.py

\#

def draw_self_loop(center, radius, facecolor='\#2693de', edgecolor='\#000000', $\backslash$

theta $1=-30$, theta $2=180)$ :

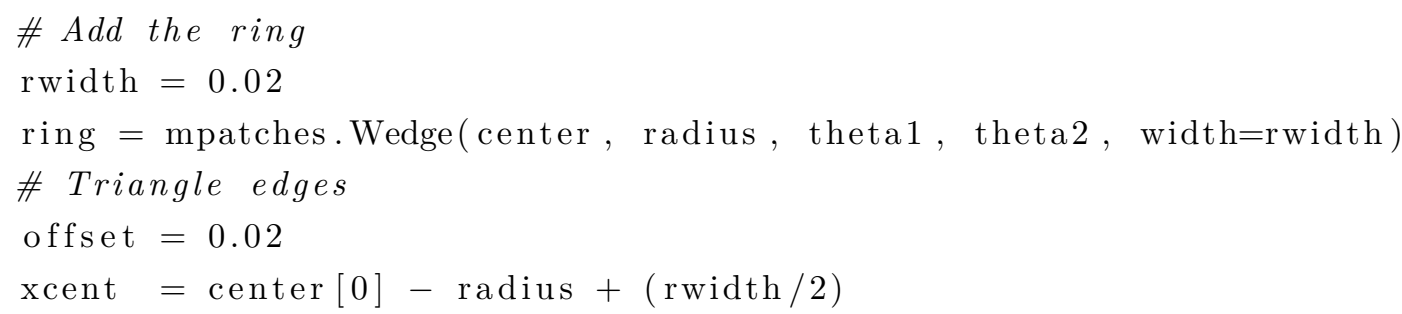




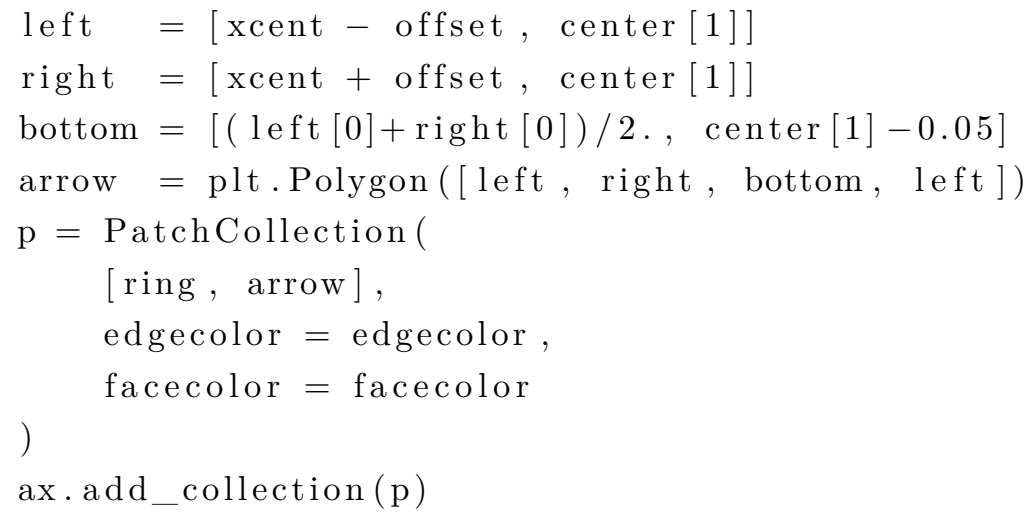

\#\%\% Read the mesh from gmsh

\#Mesh: 'c' $\rightarrow$ 4RM_Mesh_Classic.geo and 'e' $\rightarrow$ 4RM_Mesh_Expanded.geo mesh_type $=$ 'e'

input_data $=[]$

if mesh_type='c':

file $=$ open ('4RM_Mesh_Classic.geo ', ' 'r')

elif mesh_type='e':

file $=$ open('4RM_Mesh_Modified.geo', , 'r')

file $=$ file.readlines ()

for line in file:

line $=$ re.findall $\left(\mathrm{r}^{\prime \prime}[-+] ? \backslash \mathrm{d} * \backslash \cdot \backslash \mathrm{d}+\mid \backslash \mathrm{d}+"\right.$, line $)$

if line $!=[]$ :

input_data.append (line [0])

\#\% Input parameters

\# Fluid Constant Density

rho $=887.2$

\# Fluid Constant Viscosity

$\mathrm{mu}=34 \mathrm{e}-3$

\# Cylinder's radius a

$\mathrm{R}=$ float (input_data $[0]$ )

\# Distance between centers of rollers

$\mathrm{b}=$ float (input_data [1])

\# Gap between rollers

$\mathrm{d}=2 *(\mathrm{~b}-\mathrm{R})$ 


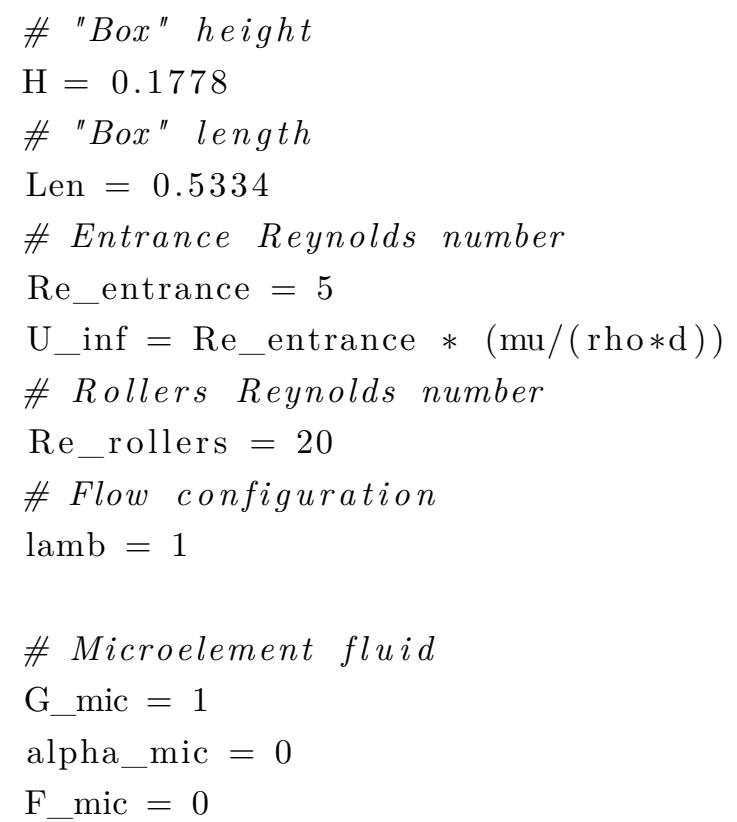

\#\%\% Flow configuration

\# Cylinder's center point

ญ \#UP

$\mathrm{x} \_$center_UR $=\mathrm{b}$

$\mathrm{y} \_$center_UR $=\mathrm{b}$

\# UL

$\mathrm{x} \_$center_UL $=-\mathrm{b}$

$\mathrm{y} \_$center_UL $=\mathrm{b}$

\# $B L$

$\mathrm{x} \_$center_BL $=-\mathrm{b}$

$\mathrm{y} \_$center_BL $=-\mathrm{b}$

\# BR

$\mathrm{x} \_$center_BR $=\mathrm{b}$

$\mathrm{y} \_$center_BR $=-\mathrm{b}$

\# Cylinder's angular velocity

\# Pure extensional flow $\rightarrow$ lambda $=1$

\# Simple shear flow $\rightarrow>$ lambda $=0$

\# Rigid body motion $\rightarrow$ lambda $=-1$

if $l a m b==-1$ :

omg_UR $=\mathrm{Re} \_$rollers $*(\mathrm{mu} /(\operatorname{rho} * \mathrm{~d} * \mathrm{R}))$

omg_UL $=$ omg UR

omg $\mathrm{BL}=$ omg UR

omg $\mathrm{BR}=$ omg UR

elif $\operatorname{lamb}==-0.5$ :

omg_UR $=\mathrm{Re} \_$rollers $*(\mathrm{mu} /(\operatorname{rho} * \mathrm{~d} * \mathrm{R}))$

omg_UL $=$ omg UR $/ 2$ 


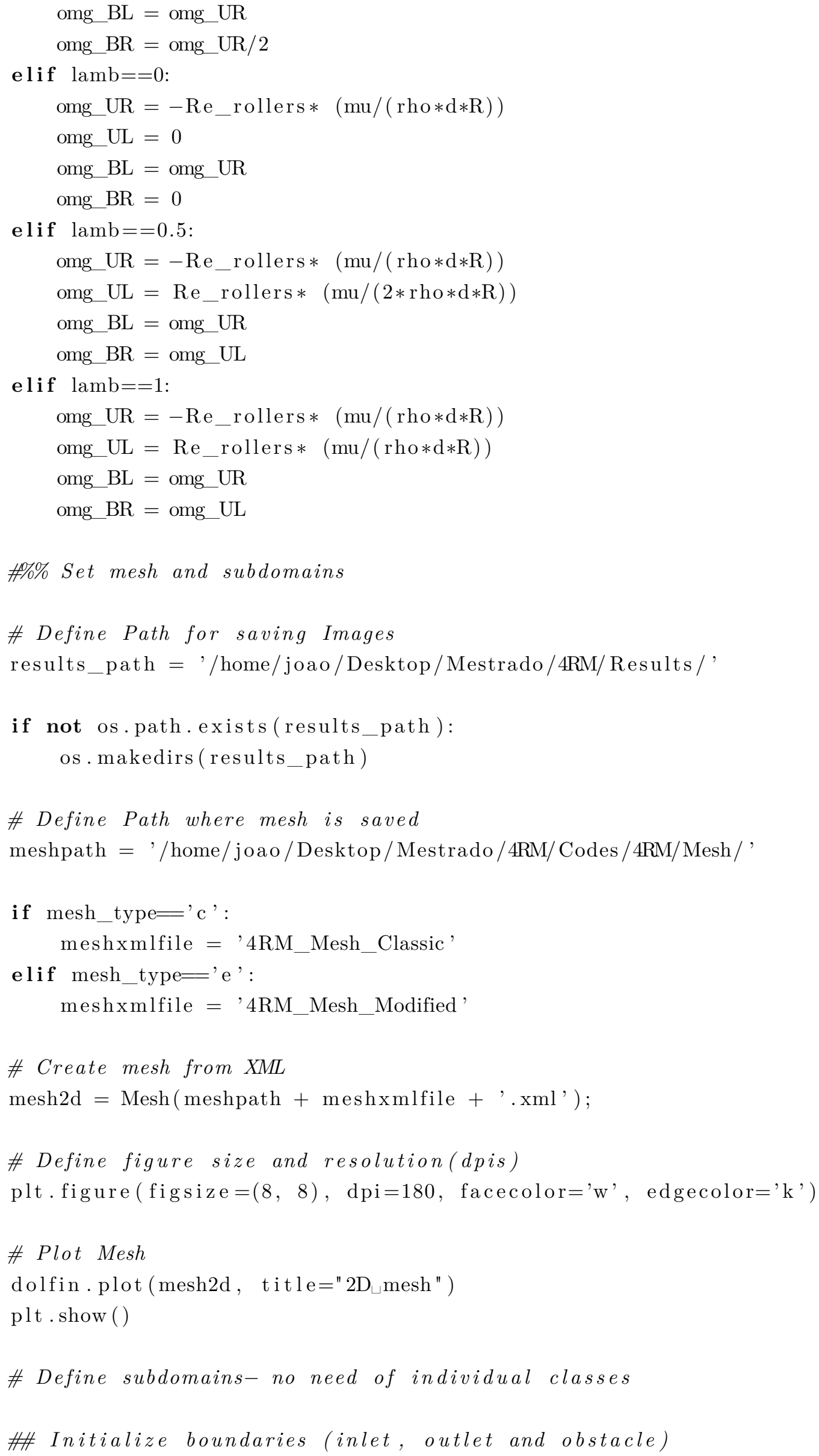


boundaries $=$ MeshFunction('size_t', mesh2d, meshpath + meshxmlfile + 1 "_facet_region.xml" );

\# Initialize subdomain (channel)

markers $=$ MeshFunction('size_t', mesh2d, meshpath + meshxmlfile $+\backslash$ '_physical_region.xml');

\# Both XMLs are automatic generated by dolfin-convert function on a \# mesh generated by script in gmsh

\# Boundaries' tags

NorthTag $=1$

WestTag $=2$

SouthTag $=3$

EastTag $=4$

UR_obstacle_NE_Tag $=5$

UR_obstacle_NW_Tag $=6$

UR_obstacle_SW_Tag $=7$

UR_obstacle_SE_Tag $=8$

UL_obstacle_NE_Tag $=9$

UL_obstacle_NW_Tag $=10$

UL_obstacle_SW_Tag $=11$

UL_obstacle_SE_Tag $=12$

BL_obstacle_NE_Tag $=13$

BL_obstacle_NW_Tag $=14$

BL_obstacle_SW_Tag $=15$

BL_obstacle_SE_Tag $=16$

BR_obstacle_NE_Tag $=17$

BR_obstacle_NW_Tag $=18$

BR_obstacle_SW_Tag $=19$

BR_obstacle_SE_Tag $=20$

fluidTag $=21$

\#\%\% Element from FEM's formulation

\# Normal Face

$\mathrm{n}=$ FacetNormal(mesh2d) \# Normal vector to mesh

$\mathrm{t}=$ as_vector $([\mathrm{n}[1],-\mathrm{n}[0]]) \quad$ \# Tangent vector to mesh

\#\# Define mixed element Function Space

shape $=\operatorname{mesh} 2 \mathrm{~d} \cdot \mathrm{ufl} \_$cell () 


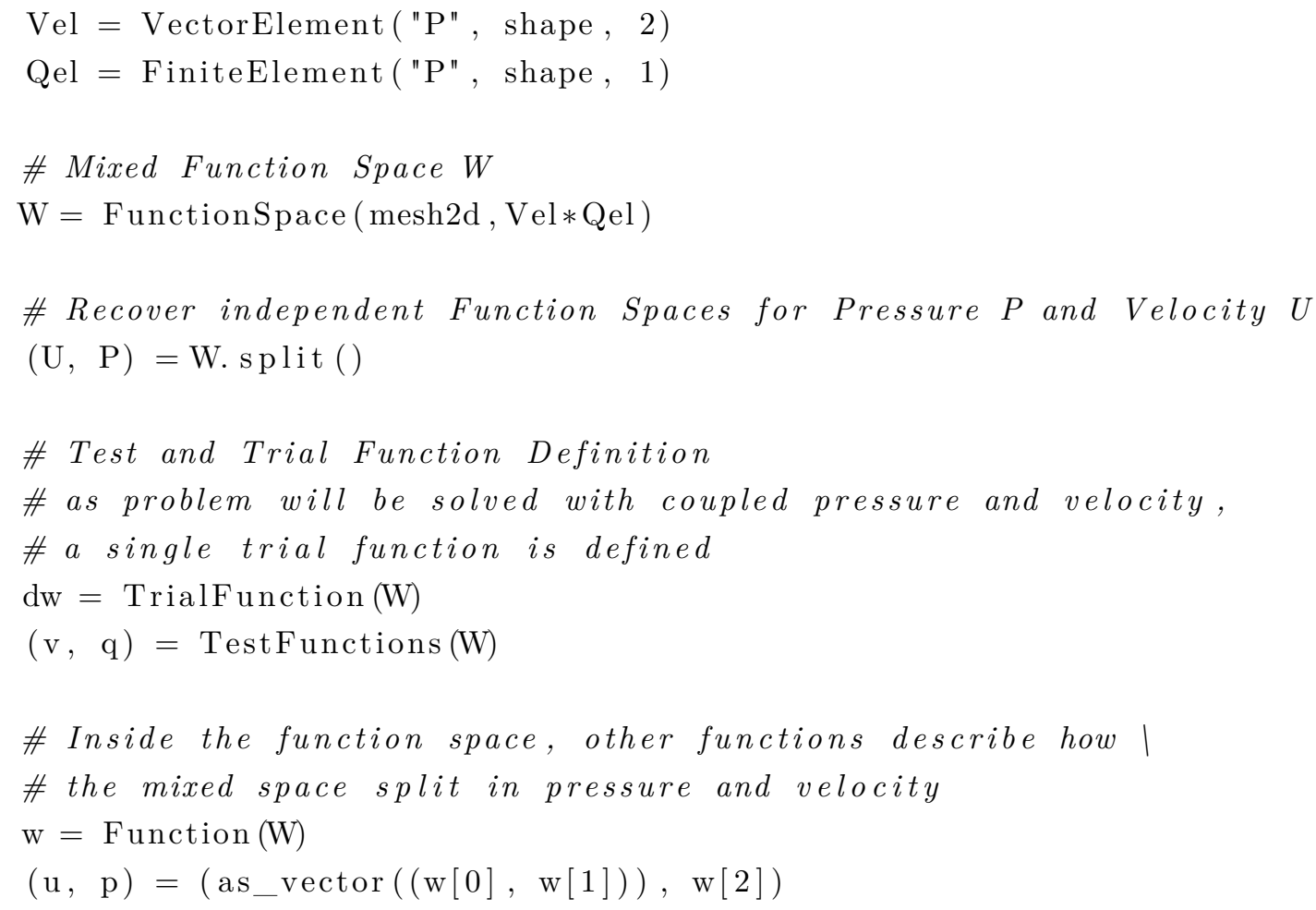

\#\% Boundary conditions

\#\# Define boundary conditions based on XML

$\mathrm{bc}=[]$

\# Upper right cylinder

$\# N E$

UR_NE_x $=$ Expression ('-omega $*\left(x[1]-y \_c\right)$ ', omega=Constant $($ omg UR $), \quad$ I y_c $=$ Constant $\left(y \_\right.$center_UR $)$, degree $\left.=2\right)$

UR_NE_y $=$ Expression ('omega $*\left(x[0]-\mathrm{x} \_c\right)$ ', omega=Constant $($ omg UR $), ।$ $\mathrm{x} \_\mathrm{c}=$ Constant $\left(\mathrm{x} \_\right.$center_UR $\left.), \operatorname{degree}=2\right)$

bc_UR_NE_x $=$ DirichletBC (U.sub $(0)$, UR_NE_x, boundaries, UR_obstacle_NE_Tag)

bc_UR_NE_y $=$ DirichletBC (U.sub(1), UR_NE_y, boundaries, UR_obstacle_NE_Tag) \# NW

UR NW_x $=$ Expression (' $-\operatorname{omega} *\left(\mathrm{x}[1]-\mathrm{y} \_\mathrm{c}\right)$ ', omega=Constant $($ omg UR $), \quad$ I y_c $=$ Constant $\left(y \_\right.$center_UR $)$, degree $\left.=2\right)$

UR_NW_y $=$ Expression ('-omega $*\left(x \_c-x[0]\right)$,, omega=Constant $($ omg UR $), \quad$ I $\mathrm{x} \_\mathrm{c}=\operatorname{Constant}\left(\mathrm{x} \_\right.$center_UR $\left.), \operatorname{degree}=2\right)$

bc_UR_NW_x = DirichletBC (U.sub (0), UR_NW_x, boundaries, UR_obstacle_NW_Tag) bc_UR_NW_y $=\operatorname{Dirichlet} B C(\mathrm{U} \cdot \mathrm{sub}(1), \mathrm{UR}$ NW_y, boundaries, UR_obstacle_NW_Tag) \# SW

UR_SW_x $=$ Expression('omega $*\left(y \_c-x[1]\right)$, omega=Constant (omg_UR), । $\mathrm{y} \_\mathrm{c}=\operatorname{Constant}\left(\mathrm{y} \_\right.$center_UR $\left.), \operatorname{degree}=2\right)$

UR_SW_y $=\operatorname{Expression}\left('-\operatorname{omega} *\left(\mathrm{x} \_c-\mathrm{x}[0]\right)\right.$, omega=Constant (omg_UR), $\mathrm{x} \_\mathrm{c}=\operatorname{Constant}\left(\mathrm{x} \_\right.$center_UR $\left.), \operatorname{degree}=2\right)$ 
bc_UR_SW_x $=$ DirichletBC (U.sub(0), UR_SW_x, boundaries, UR_obstacle_SW_Tag)

bc_UR_SW_y $=$ DirichletBC (U.sub (1), UR_SW_y, boundaries, UR_obstacle_SW_Tag)

\# SE

UR_SE_x $=$ Expression ('omega* $\left(y \_c-x[1]\right)^{\prime}$, omega=Constant (omg_UR $), \quad \mid$

y_c=Constant (y_center_UR $),$ degree $=2$ )

UR_SE_y $=$ Expression ('omega $*\left(x[0]-x \_c\right)$ ', omega=Constant $($ omg_UR $)$,

$\mathrm{x} \_\mathrm{c}=$ Constant $\left(\mathrm{x} \_\right.$center_UR $)$, degree $\left.=2\right)$

bc_UR_SE_x $=$ DirichletBC (U.sub (0), UR_SE_x, boundaries, UR_obstacle_SE_Tag)

bc_UR_SE_y $=$ DirichletBC (U.sub (1), UR_SE_y, boundaries, UR_obstacle_SE_Tag)

\# Upper left cylinder

\# NE

UL_NE_x $=$ Expression (' - omega $*\left(x[1]-y \_c\right) '$, omega=Constant (omg_UL $), ।$

y_c=Constant (y_center_UL), degree $=2$ )

UL_NE_y $=$ Expression ('omega $*\left(\mathrm{x}[0]-\mathrm{x} \_\mathrm{c}\right)$ ', omega=Constant (omg_UL), ।

$\mathrm{x} \_c=$ Constant $\left(\mathrm{x} \_\right.$center_UL $)$, degree $\left.=2\right)$

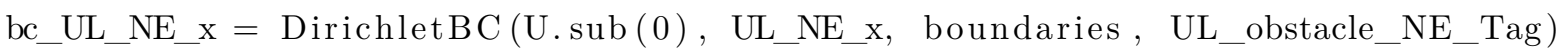

bc_UL_NE_y $=$ DirichletBC (U.sub (1), UL_NE_y, boundaries, UL_obstacle_NE_Tag)

\# NW

UL_NW_x $=$ Expression ('-omega $*\left(\mathrm{x}[1]-\mathrm{y} \_\mathrm{c}\right)$ ', omega=Constant (omg_UL), ।

y_c $=$ Constant $\left(y \_c e n t e r \_U L\right)$, degree $\left.=2\right)$

UL_NW_y $=$ Expression ('-omega $*\left(\mathrm{x} \_\mathrm{c}-\mathrm{x}[0]\right)^{\prime}$, , omega=Constant (omg_UL), ।

$\mathrm{x} \_c=$ Constant $\left(\mathrm{x} \_\right.$center_UL $)$, degree $\left.=2\right)$

bc_UL_NW_x $=$ DirichletBC (U.sub (0), UL_NW_x, boundaries, UL_obstacle_NW_Tag)

bc_UL_NW_y $=$ DirichletBC (U.sub (1), UL_NW_y, boundaries, UL_obstacle_NW_Tag)

$\# S W$

UL_SW_x $=$ Expression ('omega $*\left(y \_c-x[1]\right) '$, omega=Constant (omg_UL),

y_c $=$ Constant (y_center_UL), degree $=2$ )

UL_SW_y $=$ Expression ('-omega $*\left(x \_c-x[0]\right)$ ', omega=Constant (omg_UL), ।

$\mathrm{x} \_c=$ Constant $\left(\mathrm{x} \_\right.$center_UL $)$, degree $\left.=2\right)$

bc_UL_SW_x $=$ DirichletBC(U.sub(0), UL_SW_x, boundaries, UL_obstacle_SW_Tag) bc_UL_SW_y $=$ DirichletBC(U.sub(1), UL_SW_y, boundaries, UL_obstacle_SW_Tag)

\# SE

UL_SE_x $=$ Expression ('omega $*\left(y \_c-x[1]\right)$ ', omega=Constant (omg_UL), ।

y_c=Constant (y_center_UL), degree $=2$ )

UL_SE_y $=$ Expression ('omega $*\left(\mathrm{x}[0]-\mathrm{x} \_\mathrm{c}\right)$ ', omega=Constant (omg_UL), । $\mathrm{x} \_c=$ Constant $\left(\mathrm{x} \_\right.$center_UL $)$, degree $=2$ )

bc_UL_SE_x $=$ DirichletBC (U.sub (0), UL_SE_x, boundaries, UL_obstacle_SE_Tag) bc_UL_SE_y $=$ DirichletBC (U.sub (1), UL_SE_y, boundaries, UL_obstacle_SE_Tag)

\# Bottom left cylinder

\# NE

$\mathrm{BL} \_\mathrm{NE} \_\mathrm{x}=$ Expression $\left('-\right.$ omega $*\left(\mathrm{x}[1]-\mathrm{y} \_\mathrm{c}\right)$ ', omega=Constant $($ omg BL $), \quad \mid$ 
$\mathrm{y} \_\mathrm{c}=\operatorname{Constant}\left(\mathrm{y} \_\right.$center_BL $\left.), \quad \operatorname{degree}=2\right)$

BL_NE_y $=$ Expression ('omega* $\left(x[0]-\mathrm{x} \_c\right)$ ', omega=Constant $($ omg BL $), ।$

$\mathrm{x} \_\mathrm{c}=\operatorname{Constant}\left(\mathrm{x} \_\right.$center_BL $\left.), \operatorname{degree}=2\right)$

bc_BL_NE_x $=\operatorname{Dirichlet} B C\left(\mathrm{U} \cdot \operatorname{sub}(0), \mathrm{BL} \_\right.$NE_x, boundaries, BL_obstacle_NE_Tag) bc_BL_NE_y $=\operatorname{Dirichlet} B C(\mathrm{U} . \mathrm{sub}(1)$, BL_NE_y, boundaries, BL_obstacle_NE_Tag)

$\# N W$

BL_NW_x $=$ Expression ('-omega $*\left(x[1]-y \_c\right)$ ', omega=Constant $($ omg_BL $), \quad$

$\mathrm{y} \_\mathrm{c}=\operatorname{Constant}\left(\mathrm{y} \_\right.$center_BL $)$, degree $\left.=2\right)$

BL_NW_y $=$ Expression ('-omega $*\left(x \_c-x[0]\right)$,, omega=Constant $($ omg_BL $), ।$

$\mathrm{x} \_\mathrm{c}=\operatorname{Constant}\left(\mathrm{x} \_\right.$center_BL $\left.), \operatorname{degree}=2\right)$

bc_BL_NW_x = DirichletBC (U.sub $(0)$, BL_NW_x, boundaries, BL_obstacle_NW_Tag) bc_BL_NW_y $=\operatorname{Dirichlet} B C\left(\mathrm{U} . \operatorname{sub}(1), \mathrm{BL} \_\mathrm{NW} y\right.$, boundaries, BL_obstacle_NW_Tag)

\# SW

BL_SW_x $=$ Expression ('omega $*\left(y \_c-x[1]\right)$ ', omega=Constant (omg_BL), । $\mathrm{y} \_\mathrm{c}=\operatorname{Constant}\left(\mathrm{y} \_\right.$center_BL $\left.), \operatorname{degree}=2\right)$

BL_SW_y $=$ Expression ('-omega $*\left(x \_c-x[0]\right)$, $, \operatorname{omega}=\operatorname{Constant}($ omg BL $), ।$ $\mathrm{x} \_\mathrm{c}=\operatorname{Constant}\left(\mathrm{x} \_\right.$center_BL $\left.), \operatorname{degree}=2\right)$

bc_BL_SW_x = DirichletBC (U.sub $(0)$, BL_SW_x, boundaries, BL_obstacle_SW_Tag) bc_BL_SW_y $=\operatorname{Dirichlet} B C(U . \operatorname{sub}(1)$, BL_SW_y, boundaries, BL_obstacle_SW_Tag)

\# SE

$\mathrm{BL} \_\mathrm{SE} \_\mathrm{x}=\operatorname{Expression}\left(\operatorname{lomega}\left(\mathrm{y} \_\mathrm{c}-\mathrm{x}[1]\right)\right.$ ', omega=Constant $(\operatorname{omg} \mathrm{BL})$, $\mathrm{y} \_\mathrm{c}=\operatorname{Constant}\left(\mathrm{y} \_\right.$center_BL $\left.), \quad \operatorname{degree}=2\right)$

BL_SE_y $=$ Expression ('omega $*\left(x[0]-x \_c\right)$ ', omega=Constant $($ omg_BL $), ।$ $\mathrm{x} \_\mathrm{c}=\operatorname{Constant}\left(\mathrm{x} \_\right.$center_BL $\left.), \operatorname{degree}=2\right)$

bc_BL_SE_x = DirichletBC (U.sub (0), BL_SE_x, boundaries, BL_obstacle_SE_Tag) bc_BL_SE_y $=\operatorname{Dirichlet} B C\left(\mathrm{U} . \mathrm{sub}(1), \mathrm{BL} \_\mathrm{SE} \_\mathrm{y}\right.$, boundaries, BL_obstacle_SE_Tag)

\# Bottom right cylinder

\# NE

BR_NE_x $=$ Expression ('-omega $*\left(x[1]-y \_c\right)$ ', omega=Constant $($ omg_BR $), \quad \mid$

$\mathrm{y} \_\mathrm{c}=\operatorname{Constant}\left(\mathrm{y} \_\right.$center_BR $\left.), \operatorname{degree}=2\right)$

$\mathrm{BR}$ NE_y $=$ Expression $\left(' \operatorname{omega} *\left(\mathrm{x}[0]-\mathrm{x} \_\mathrm{c}\right)\right.$ ', omega=Constant $($ omg BR $)$, $\mathrm{x} \_\mathrm{c}=\operatorname{Constant}\left(\mathrm{x} \_\right.$center_BR$\left.), \operatorname{degree}=2\right)$

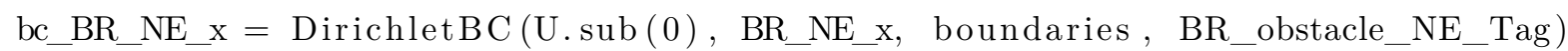
bc_BR_NE_y $=\operatorname{Dirichlet} B C(\mathrm{U} . \mathrm{sub}(1), \mathrm{BR}$ NE_y, boundaries, BR_obstacle_NE_Tag)

$\# N W$

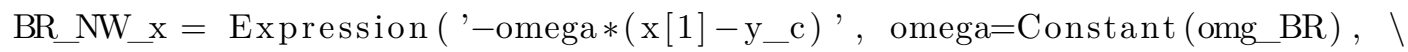

$\mathrm{y} \_\mathrm{c}=\operatorname{Constant}\left(\mathrm{y} \_\right.$center_BR $\left.), \operatorname{degree}=2\right)$

BR_NW_y $=$ Expression ('-omega $*\left(x \_c-x[0]\right)$ ', omega=Constant $($ omg BR $), ।$ $\mathrm{x} \_\mathrm{c}=\operatorname{Constant}\left(\mathrm{x} \_\right.$center_BR $\left.), \operatorname{degree}=2\right)$

bc_BR_NW_x = DirichletBC (U.sub $(0)$, BR_NW_x, boundaries, BR_obstacle_NW_Tag)

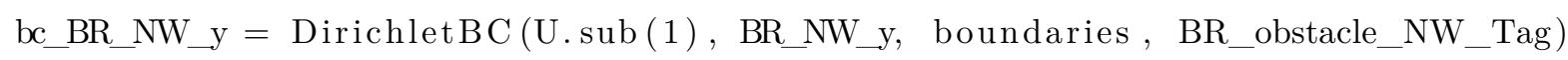




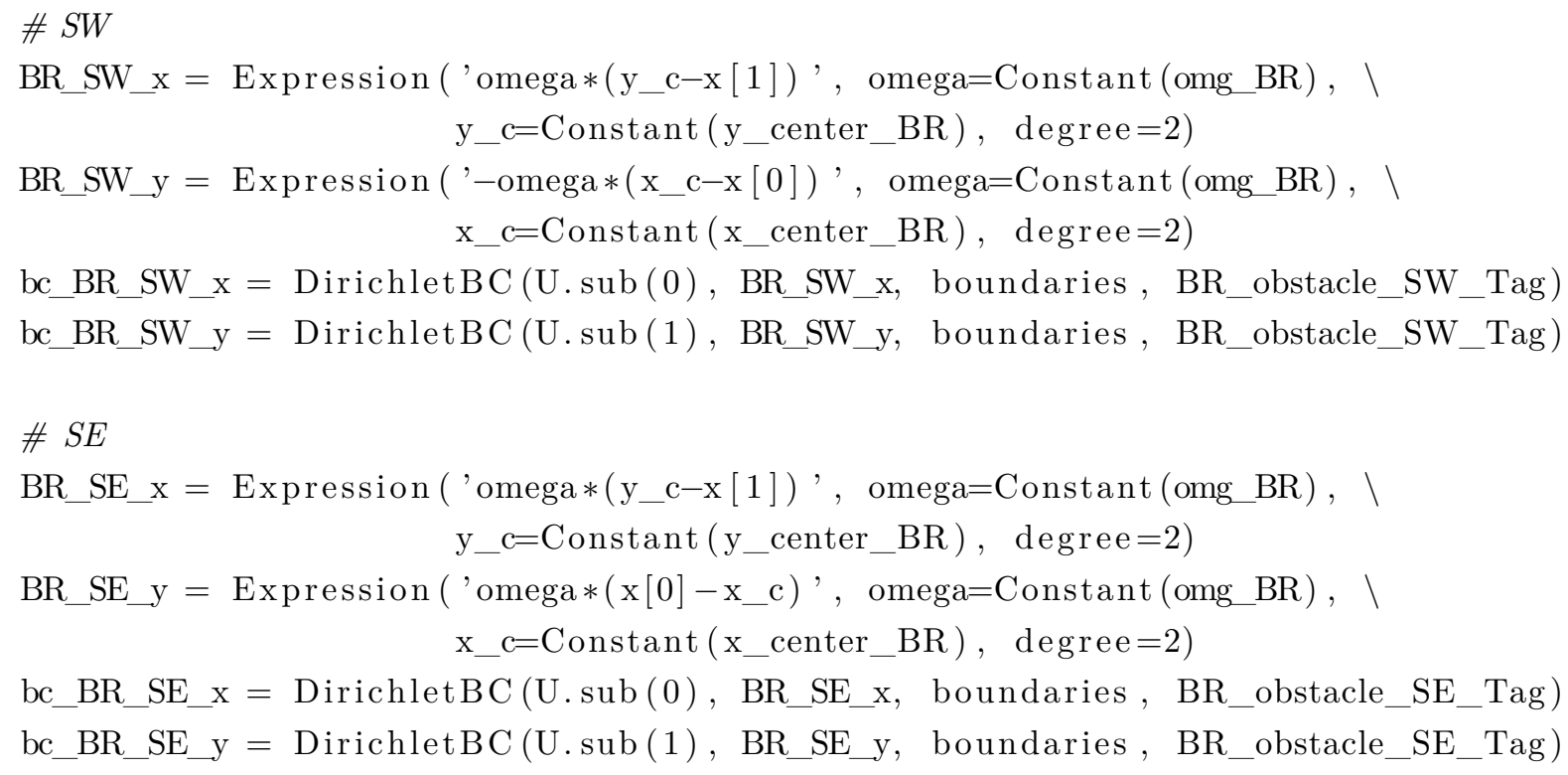

bc . append (bc_UR_NE_x)

bc . append (bc_UR_NE_y)

bc.append (bc_UR_NW_x)

bc.append (bc_UR_NW_y)

$b c$.append (bc_UR_SW_x)

bc.append (bc_UR_SW_y)

bc . append (bc_UR_SE_x)

bc.append (bc_UR_SE_y)

bc . append (bc_UL_NE_x)

bc.append (bc_UL_NE_y)

bc.append (bc_UL_NW_x)

$\mathrm{bc}$.append (bc_UL_NW_y)

bc.append (bc_UL_SW_x)

bc .append (bc_UL_SW_y)

bc . append (bc_UL_SE_x)

$\mathrm{bc}$. append (bc_UL_SE_y)

bc. append (bc_BL_NE_x)

$b c$.append (bc_BL_NE_y)

$b c$.append (bc_BL_NW_x)

$\mathrm{bc}$. append (bc_BL_NW_y)

bc.append (bc_BL_SW_x)

bc.append (bc_BL_SW_y)

bc. append (bc_BL_SE_x)

$\mathrm{bc}$. append (bc_BL_SE_y)

bc.append (bc_BR_NE_x)

$b c$. append (bc_BR_NE_y)

$b c$. append (bc_BR_NW_x) 
$b c \cdot a p p e n d\left(b c \_B R \_N W \_y\right)$
$b c \cdot a p p e n d\left(b c \_B R \_S W \_x\right)$
$b c \cdot a p p e n d\left(b c \_B R \_S W \_y\right)$
$b c \cdot a p p e n d\left(b c \_B R \_S E \_x\right)$
$b c . a p p e n d\left(b c \_B R \_S E \_y\right)$

if mesh_type=' c':

bc_W = DirichletBC $(\mathrm{U} \cdot \operatorname{sub}(0)$, Constant $(0.0)$, boundaries, WestTag $)$

bc_E $=$ DirichletBC (U.sub $(0)$, Constant $(0.0)$, boundaries, EastTag)

bc_N $=$ DirichletBC(U.sub (1), Constant (0.0), boundaries, NorthTag)

bc_S $=$ DirichletBC (U.sub (1), Constant (0.0), boundaries, SouthTag)

bc . append (bc_W)

bc.append (bc_E)

$\mathrm{bc}$. append (bc_N)

$\mathrm{bc} . \operatorname{append}\left(\mathrm{bc} \_\mathrm{S}\right)$

elif mesh_type=' e' :

bc_W = DirichletBC $\left(\mathrm{U}\right.$, Constant $\left(\left(\mathrm{U}_{\text {_inf }}, 0.0\right)\right)$, boundaries, WestTag $)$

bc_N $=$ DirichletBC(U.sub(1), Constant (0.0), boundaries, NorthTag)

bc_S = DirichletBC(U.sub(1), Constant (0.0), boundaries, SouthTag)

bc . append (bc_W)

bc . append ( bc_N)

bc.append (bc_S)

\#\%\% Week formulation

\#\# Define variational forms

\# Define new measure with associated subdomains

$\mathrm{ds}=$ Measure ('ds', domain=mesh2d, subdomain_data=boundaries )

\# Momentum Equation

\# Inertia Term \# Viscous Force Term

\# Pressure Force Term

$\mathrm{a}=\left(\operatorname{inner}\left(\operatorname{dot}\left(\mathrm{u}, \operatorname{nabla} \_\operatorname{grad}(\mathrm{u})\right), \mathrm{v}\right)+(\operatorname{mu} / \operatorname{rho}) * \operatorname{inner}(\operatorname{grad}(\mathrm{u}), \operatorname{grad}(\mathrm{v})) \backslash\right.$ - $(1 / \mathrm{rho}) * \operatorname{div}(\mathrm{v}) * \mathrm{p}) * \operatorname{dx}()$

$\mathrm{L}=0$

\# Continuity Equation

$\mathrm{a}=\mathrm{a}+(\mathrm{q} * \operatorname{div}(\mathrm{u})) * \mathrm{dx}()$

$\mathrm{L}=\mathrm{L}+0$

$\mathrm{F}=\mathrm{a}-\mathrm{L}$

\# Calculate Jacobian Matrix

$\mathrm{J}=\operatorname{derivative}(\mathrm{F}, \mathrm{w}, \mathrm{dw})$ 


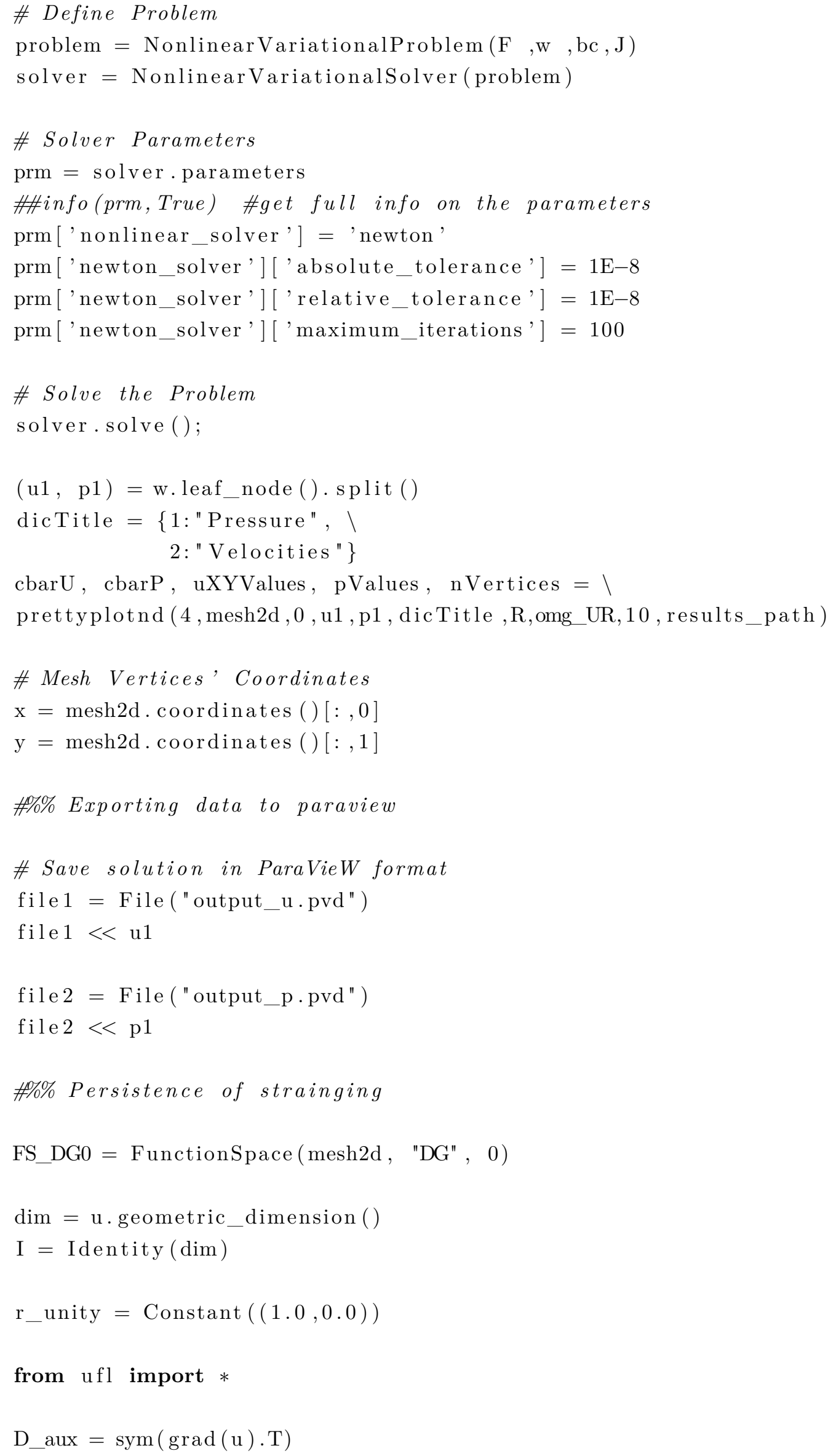




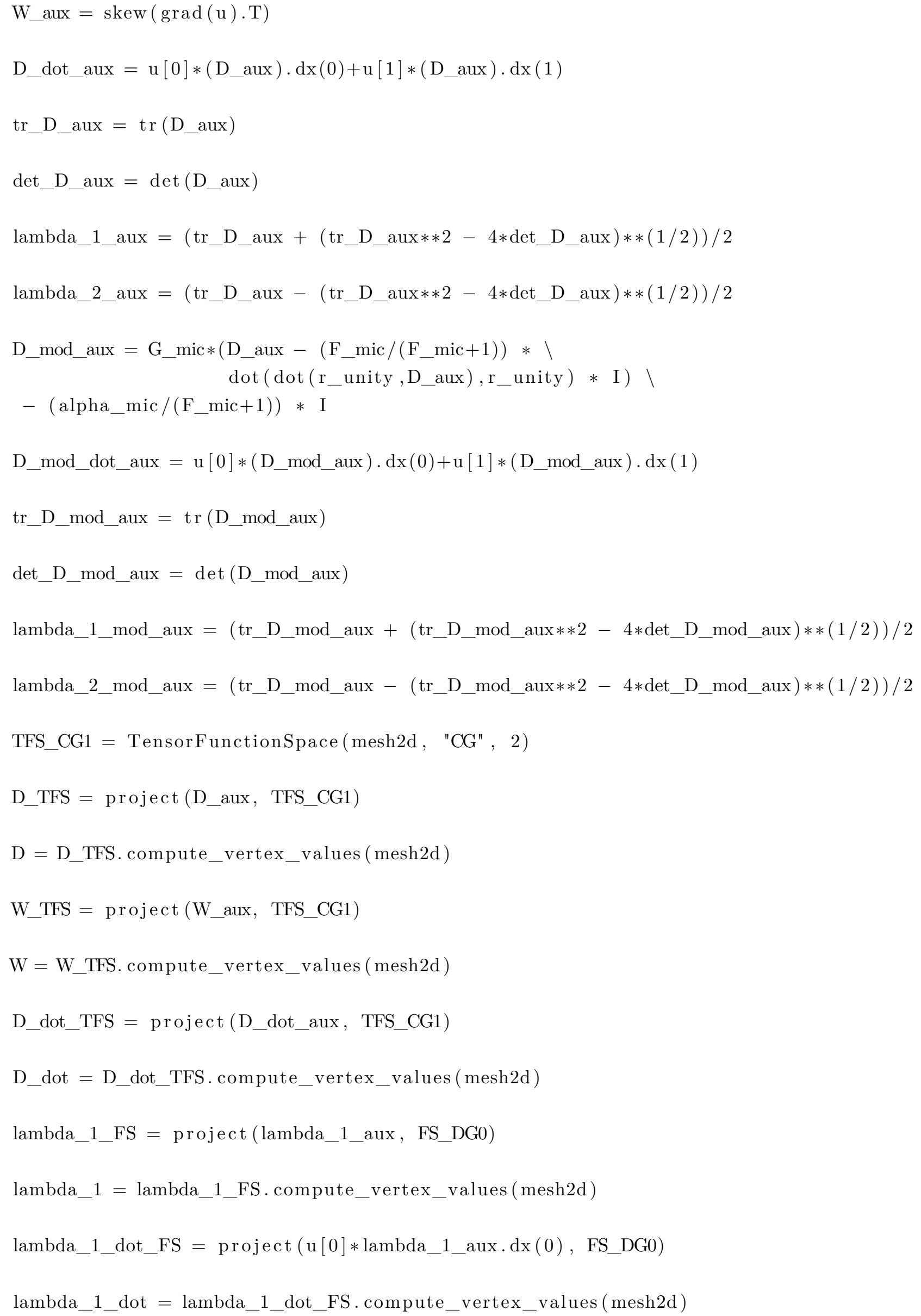


lambda_2_FS $=\operatorname{project}($ lambda_2_aux, FS_DG0 $)$

lambda_2 = lambda_2_FS.compute_vertex_values (mesh2d)

lambda_2_dot_FS $=\operatorname{project}\left(\mathrm{u}[1] * \operatorname{lambda\_ } 2 \_\right.$aux. $\mathrm{dx}(1)$, FS_DG0 $)$

lambda_2_dot $=$ lambda_2_dot_FS.compute_vertex_values $(\operatorname{mesh} 2 \mathrm{~d})$

D_mod_TFS $=\operatorname{project}\left(\mathrm{D} \_\right.$mod_aux, TFS_CG1 $)$

D_mod $=$ D_mod_TFS.compute_vertex_values $(\operatorname{mesh} 2 \mathrm{~d})$

$\mathrm{W} \_\mathrm{TFS}=\operatorname{project}\left(\mathrm{W} \_\right.$aux, TFS_CG1 $)$

$\mathrm{W}=\mathrm{W} \_$TFS. compute_vertex_values $(\operatorname{mesh} 2 \mathrm{~d})$

D_mod_dot_TFS $=\operatorname{project}\left(\mathrm{D} \_\right.$mod_dot_aux, TFS_CG1)

D_mod_dot $=$ D_mod_dot_TFS.compute_vertex_values (mesh2d)

lambda_1_mod_dot_FS $=\operatorname{project}\left(\mathrm{u}[0] * \operatorname{lambda} \_1 \_\right.$mod_aux.dx $(0)$, FS_DG0 $)$

lambda_1_mod_dot $=$ lambda_1_mod_dot_FS.compute_vertex_values $(\operatorname{mesh} 2 \mathrm{~d})$

lambda_2_mod_dot_FS $=\operatorname{project}\left(\mathrm{u}[1] * \operatorname{lambda} \_2 \_\bmod \_\right.$aux.dx $(1)$, FS_DG0 $)$

lambda_2_mod_dot $=$ lambda_2_mod_dot_FS.compute_vertex_values $(\operatorname{mesh} 2 \mathrm{~d})$

$\mathrm{nVertices}=\operatorname{len}(\mathrm{x})$

$\operatorname{print}(\operatorname{len}(\mathrm{x}))$

\# Order dimension of the problem

$\mathrm{N}=2 \# 2 D$

D_11_nodal $=[]$

D_12_nodal $=[]$

D_21_nodal $=$ []

D_22_nodal $=[]$

W_11_nodal $=$ []

$\mathrm{W} \_12 \_$nodal $=[]$

$\mathrm{W} \_21 \_$nodal $=[]$

$\mathrm{W} \_22 \_$nodal $=$[]

D_dot_11_nodal $=$ []

D_dot_12_nodal $=[]$ 

D_dot_21_nodal $=[]$
D_dot_22_nodal $=[]$
D_mod_11_nodal $=[]$
D_mod_12_nodal $=[]$
D_mod_21_nodal $=[]$
D_mod_22_nodal $=[]$
D_mod_dot_11_nodal $=[]$
D__mod_dot_12_nodal $=[]$
D_mod_dot_21_nodal $=[]$
D_mod_dot_22_nodal $=[]$

for i in range $(0,(\mathrm{~N} * * 2) * \mathrm{nVertices})$ :

if $\mathrm{i}>=0$ and $\mathrm{i}<\mathrm{nVertices:}$

D_11_nodal .append (D [ i ] )

W_11_nodal . append (W[i ] )

D_dot_11_nodal . append (D_dot [i ] )

D_mod_11_nodal . append (D_mod [i ] )

D_mod_dot_11_nodal .append (D_mod_dot [i ])

elif $\mathrm{i}>=n$ Vertices and $\mathrm{i}<2 * \mathrm{nVertices}$ :

D_12_nodal .append (D [ i ] )

W_12_nodal . append (W[i ] )

D_dot_12_nodal . append (D_dot [i ] )

D_mod_12_nodal. append $\left(\mathrm{D} \_\bmod [\mathrm{i}]\right)$

D_mod_dot_12_nodal . append (D_mod_dot [i ] )

elif $\mathrm{i}>=2 * \mathrm{n}$ Vertices and $\mathrm{i}<3 * \mathrm{nVertices}$ :

D_21_nodal.append (D [i ] )

W_21_nodal .append (W[i ] )

D_dot_21_nodal.append (D_dot [i ] )

D_mod_21_nodal . append (D_mod [i ] )

D_mod_dot_21_nodal.append (D_mod_dot [i ] )

elif $\mathrm{i}>=3 * \mathrm{n}$ Vertices and $\mathrm{i}<4 * \mathrm{nVertices}$ :

D_22_nodal.append (D [ i ] )

W_22_nodal.append (W[i ] )

D_dot_22_nodal .append (D_dot [i ] )

D_mod_22_nodal. append (D_mod [i ] )

D_mod_dot_22_nodal . append (D_mod_dot [i ] )

else :

print (' error')

D_nodal $=[]$

$\mathrm{W} \_$nodal $=[]$

D_dot_nodal $=[]$

lambda_dot_nodal $=[]$

eigenvalues_nodal $=[]$

eigenvectors_nodal $=[]$ 


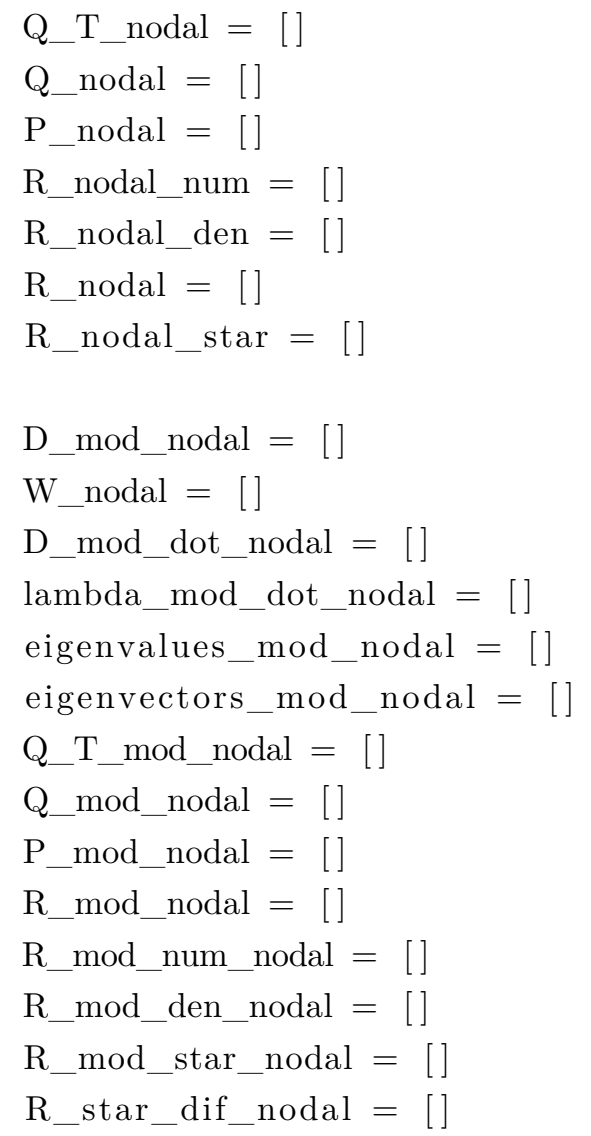

for $i$ in range $(0, n$ Vertices $)$ :

D_nodal.append (np.matrix ([[D_11_nodal [i], D_12_nodal [i ] ] , ।

[D_21_nodal [i], D_22_nodal [i ] ] ]))

W_nodal.append (np.matrix ([[W_11_nodal[i], W_12_nodal[i ] ] , ।

[W_21_nodal [i ] , W_22_nodal [i ] ] ]) )

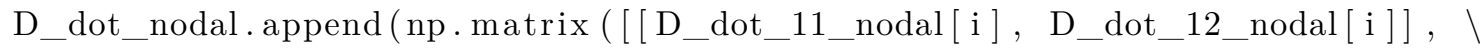

[D_dot_21_nodal[i], D_dot_22_nodal[i ] ] ]))

lambda_dot_nodal.append (np.matrix ([ [ lambda_1_dot $[\mathrm{i}], 0], \quad\left[0, \operatorname{lambda\_ 2\_ dot[i]]]))}\right.$ eig_va_nodal, eig_ve_nodal = la.eig $\left(D_{\text {_nnodal }[\mathrm{i}])}\right.$

eigenvalues_nodal.append (eig_va_nodal)

eigenvectors_nodal append (eig_ve_nodal)

Q_T_nodal.append (eigenvectors_nodal [i ] )

Q_nodal.append ( $\mathrm{Q} \_\mathrm{T} \_$nodal $\left.\left.[\mathrm{i}]\right) . \mathrm{T}\right)$

$\mathrm{P} \_$nodal .append $\left(\mathrm{D} \_\right.$nodal $[\mathrm{i}] * \mathrm{~W} \_$nodal $[\mathrm{i}]-\mathrm{W} \_$nodal $[\mathrm{i}] * \mathrm{D} \_$nodal $[\mathrm{i}]+1$

D_dot_nodal [i ] - Q_T_nodal [i ] * lambda_dot_nodal [i ] * Q_nodal [i ] )

$\mathrm{R} \_$nodal .append $\left(\left(\operatorname{sqrt}\left(0.5 *\left(\mathrm{np} \cdot \operatorname{trace}\left(\mathrm{P} \_\right.\right.\right.\right.\right.$nodal $\left.\left.\left.\left.[\mathrm{i}] * \mathrm{P}_{\text {_nodal }}[\mathrm{i}]\right)\right)\right)\right) ।$

/np.trace $\left(D_{\text {_nnodal }}[\mathrm{i}] * \mathrm{D} \_\right.$nodal $\left.\left.[\mathrm{i}]\right)\right)$

$\mathrm{R} \_$nodal_num.append $\left(\operatorname{sqrt}\left(0.5 *\left(\operatorname{np} . \operatorname{trace}\left(\mathrm{P} \_\right.\right.\right.\right.$nodal $[\mathrm{i}] * \mathrm{P} \_$nodal $\left.\left.\left.\left.[\mathrm{i}]\right)\right)\right)\right)$

$\mathrm{R} \_$nodal_den. $\operatorname{append}\left(\mathrm{np} . \operatorname{trace}\left(\mathrm{D} \_\right.\right.$nodal $[\mathrm{i}] * \mathrm{D} \_$nodal $\left.\left.[\mathrm{i}]\right)\right)$

R_nodal_star.append $\left(\left(1-\mathrm{R} \_\right.\right.$nodal $\left.[\mathrm{i}]\right) /\left(1+\mathrm{R} \_\right.$nodal $\left.\left.[\mathrm{i}]\right)\right)$

D_mod_nodal .append (np.matrix ([ [D_mod_11_nodal [i], D_mod_12_nodal [i $]], \backslash$

[D_mod_21_nodal[i], D_mod_22_nodal[i ] ] ]))

D_mod_dot_nodal.append (np.matrix ( [ [ D_mod_dot_11_nodal [i ] , ।

D_mod_dot_12_nodal[i]], । 


$$
\begin{aligned}
& \text { [D_mod_dot_21_nodal [i ] , । } \\
& \text { D_mod_dot_22_nodal [i ] ] ] ) ) }
\end{aligned}
$$

lambda_mod_dot_nodal.append(np.matrix ([[lambda_1_mod_dot [i], 0$], \mid$

$$
[0, \text { lambda_2_mod_dot }[\mathrm{i}]]]))
$$

eig_va_mod_nodal, eig_ve_mod_nodal = la . eig (D_mod_nodal[i])

eigenvalues_mod_nodal.append (eig_va_mod_nodal)

eigenvectors_mod_nodal.append (eig_ve_mod_nodal)

Q_T_mod_nodal.append (eigenvectors_mod_nodal [i ] )

Q_mod_nodal. append ( $\left(\mathrm{Q} \_T \_\right.$mod_nodal [i ] $\left.) . T\right)$

P_mod_nodal .append (D_mod_nodal $[\mathrm{i}] * \mathrm{~W} \_$nodal $[\mathrm{i}]-\mathrm{V}$

W_nodal $[\mathrm{i}] * \mathrm{D} \_$mod_nodal $[\mathrm{i}]+1$

D_mod_dot_nodal [i] - ।

Q_T_mod_nodal[i]*lambda_mod_dot_nodal [i ]*Q_mod_nodal[i ] )

R_mod_nodal .append $\left(\left(\operatorname{sqrt}\left(0.5 *\left(\operatorname{np} . \operatorname{trace}\left(\mathrm{P} \_\right.\right.\right.\right.\right.$mod_nodal $[\mathrm{i}] * \mathrm{P} \_$mod_nodal $\left.\left.\left.\left.[\mathrm{i}]\right)\right)\right)\right) \quad$

/np.trace (D_mod_nodal $[\mathrm{i}] * \mathrm{D} \_$mod_nodal $\left.\left.[\mathrm{i}]\right)\right)$

R_mod_num_nodal.append $\left(\operatorname{sqrt}\left(0.5 *\left(\operatorname{np} . \operatorname{trace}\left(\mathrm{P} \_\right.\right.\right.\right.$mod_nodal $[\mathrm{i}] * \mathrm{P} \_$mod_nodal $\left.\left.\left.\left.[\mathrm{i}]\right)\right)\right)\right)$

R_mod_den_nodal.append (np.trace (D_mod_nodal [i ]*D_mod_nodal [i ] ) )

$\mathrm{R} \_$mod_star_nodal. $\operatorname{append}\left(\left(1-\mathrm{R} \_\bmod \_\right.\right.$nodal $\left.[\mathrm{i}]\right) /\left(1+\mathrm{R} \_\right.$mod_nodal $\left.\left.[\mathrm{i}]\right)\right)$

R_star_dif_nodal. append $\left(\mathbf{a b s}\left(\mathbf{a b s}\left(\mathrm{R} \_\right.\right.\right.$mod_star_nodal $\left.[\mathrm{i}]\right)-\mathbf{a b s}\left(\mathrm{R} \_\right.$nodal_star $\left.\left.\left.[\mathrm{i}]\right)\right)\right)$

gamma_dot_nodal_aux $=[\mathrm{i} * 2 * \mathrm{mu}$ for i in D_dot_nodal $]$

gamma_dot_nodal $=[]$

for $i$ in range $(0, n$ Vertices $)$ :

gamma_dot_nodal.append (sqrt $((1 / 2) * \mathrm{np}$. trace (gamma_dot_nodal_aux [i] $* \backslash$ gamma_dot_nodal_aux [i ] )))

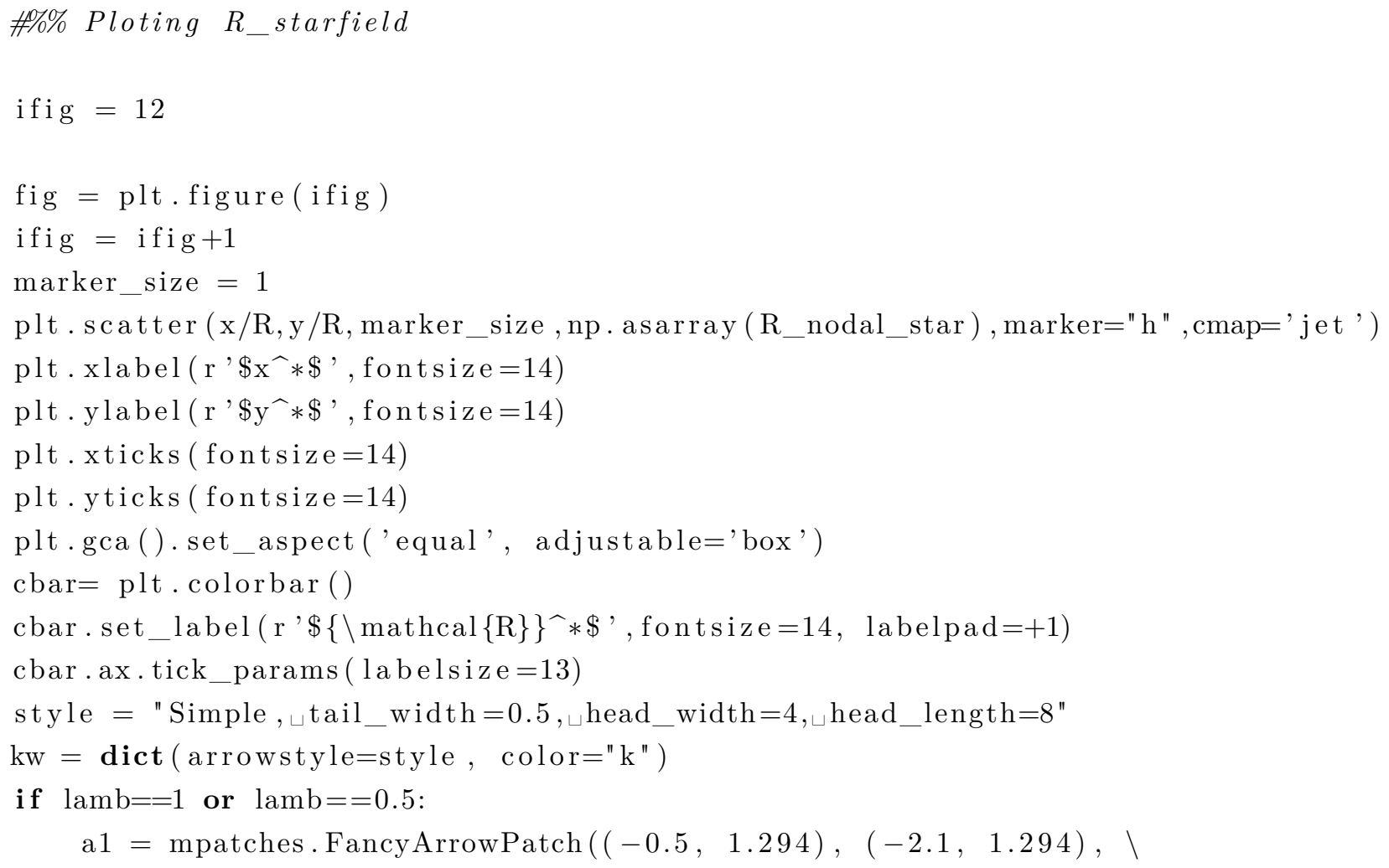




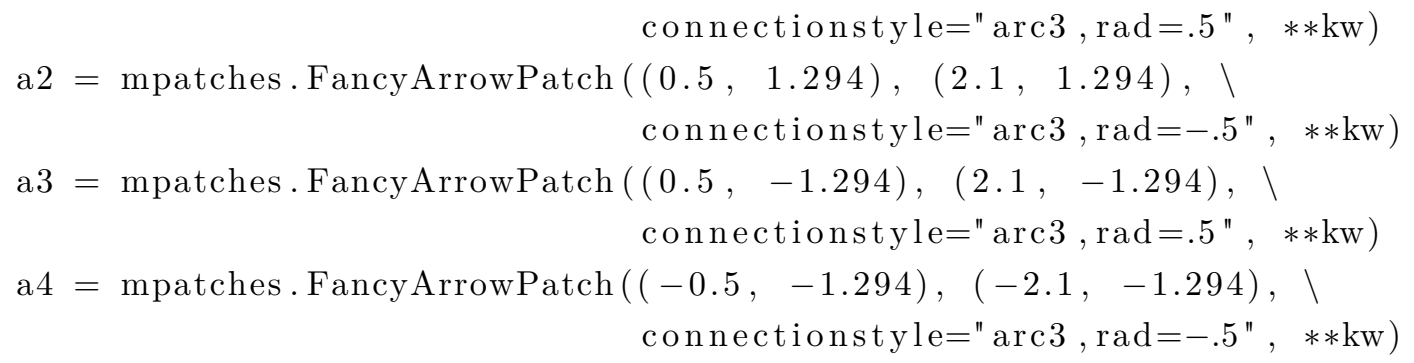

elif $\operatorname{lamb}==0$ :

$\mathrm{a} 1=$ mpatches. FancyArrowPatch $((0.5,1.294),(2.1,1.294)$, connectionstyle $\left.=" \operatorname{arc} 3, \mathrm{rad}=-.5^{\prime \prime}, * * \mathrm{kw}\right)$

$\mathrm{a} 2=$ mpatches. FancyArrowPatch $((0.5,1.294),(2.1,1.294)$, connectionstyle $=" \operatorname{arc} 3, \mathrm{rad}=-.5 ", * * \mathrm{kw})$ a3 = mpatches. FancyArrowPatch $((-0.5,-1.294),(-2.1,-1.294)$, connectionstyle $=" \operatorname{arc} 3, \operatorname{rad}=-.5 ", * * \mathrm{kw})$ $\mathrm{a} 4=$ mpatches. FancyArrowPatch $((-0.5,-1.294),(-2.1,-1.294), \backslash$ connectionstyle $=" \operatorname{arc} 3, \operatorname{rad}=-.5 ", * * \mathrm{kw})$

elif $\operatorname{lamb}==-1$ or $\operatorname{lamb}==-0.5$ :

$\mathrm{a} 1=$ mpatches. FancyArrowPatch $((-0.5,1.294),(-2.1,1.294), \backslash$ connectionstyle $=" \operatorname{arc} 3, \mathrm{rad}=.5 ", * * \mathrm{kw})$ $\mathrm{a} 2=$ mpatches. FancyArrowPatch $((2.1,1.294),(0.5,1.294)$, connectionstyle $=" \operatorname{arc} 3, \mathrm{rad}=.5 ", * * \mathrm{kw})$ a3 = mpatches. FancyArrowPatch $((0.5,-1.294),(2.1,-1.294), \backslash$ connectionstyle $=" \operatorname{arc} 3, \mathrm{rad}=.5 ", * * \mathrm{kw})$ $\mathrm{a} 4=$ mpatches. FancyArrowPatch $((-2.1,-1.294),(-0.5,-1.294), \backslash$ connectionstyle $=" \operatorname{arc} 3, \mathrm{rad}=.5 ", * * \mathrm{kw})$

for a in $[\mathrm{a} 1, \mathrm{a} 2, \mathrm{a} 3, \mathrm{a} 4]$ :

plt.gca ().add_patch (a)

plt.savefig (results_path+'R_star_field_lamb='+ $(\operatorname{str}(\operatorname{lamb})) \cdot \operatorname{replace}\left({ }^{\prime} \cdot{ }^{\prime}\right.$, 'd $\left.\left.^{\prime}\right)+{ }^{\prime} \cdot \operatorname{png},, \mathrm{dpi}=200\right)$

plt. $\operatorname{show}()$

\#\%\% Ploting R_mod_starfield

fig $=$ plt.figure(ifig)

ifig $=$ ifig +1

marker_size $=1$

plt.scatter (x/R,y/R, marker_size,np.asarray (R_mod_star_nodal $), \operatorname{marker="h",cmap='jet}$ ')

plt.xlabel ( $\mathrm{r}^{\prime} \$ \mathrm{x}^{\widehat{*}} * \$$ ', fontsize $\left.=14\right)$

plt. ylabel ( r' $\$ \mathrm{y}^{*} \$$ ', fontsize $\left.=14\right)$

plt.xticks ( fontsize $=14$ )

plt. yticks ( fontsize $=14$ )

plt.gca().set_aspect ('equal', adjustable='box')

cbar $=$ plt. colorbar ()

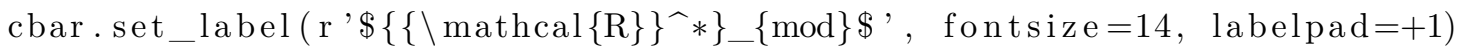

cbar.ax.tick_params ( labelsize=13)

style $=$ "Simple, $\sqcup$ tail__width $=0.5, \sqcup$ head_width $=4, \sqcup$ head_length $=8$ " 


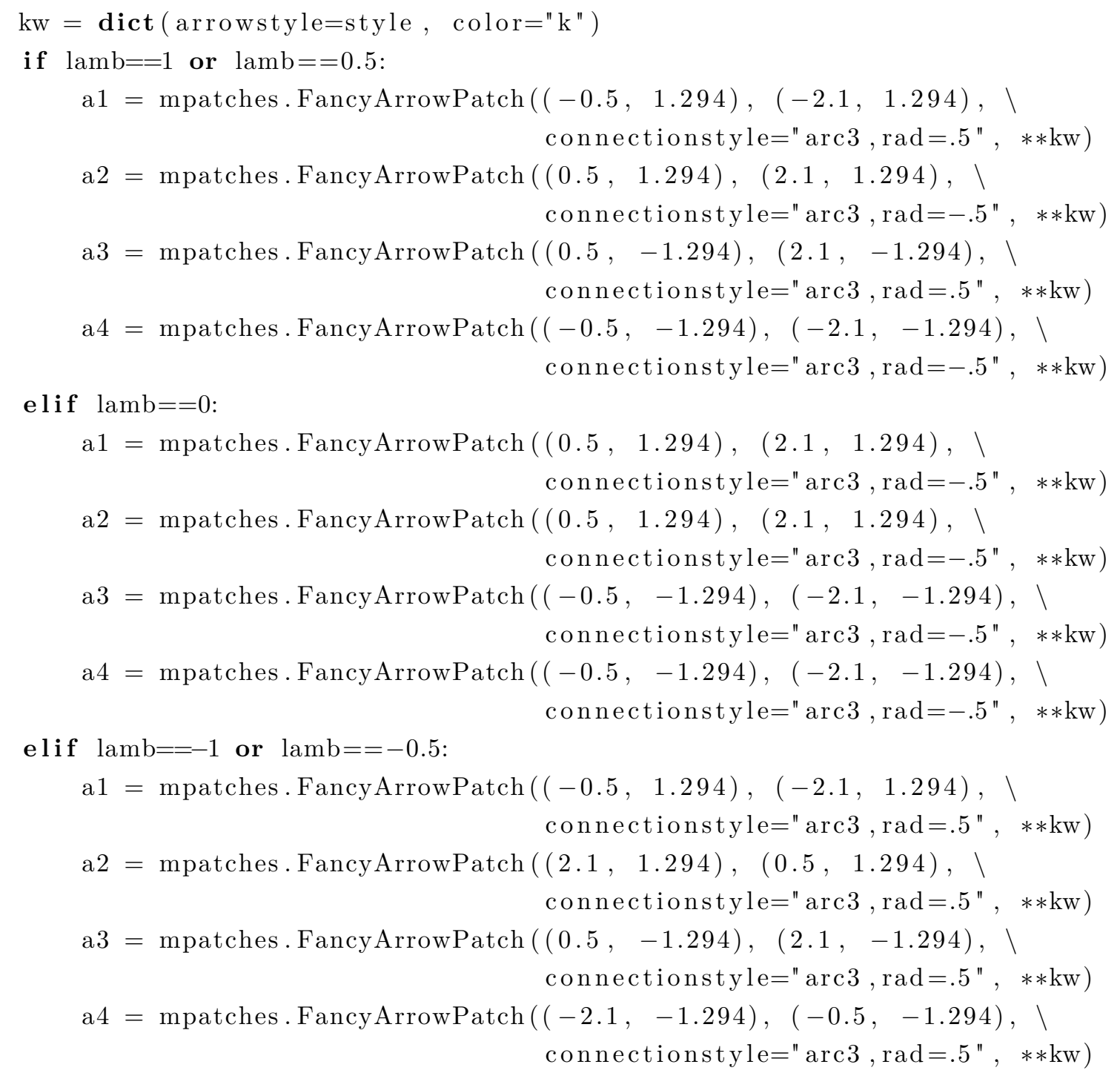

for a in $[\mathrm{a} 1, \mathrm{a} 2, \mathrm{a} 3, \mathrm{a} 4]$ :

plt.gca ().add_patch (a)

plt.savefig(results_path+'R_mod_star_field_G=' $+\left(\operatorname{str}\left(G \_\right.\right.$mic $\left.)\right)$.replace (' .' , 'd ') +

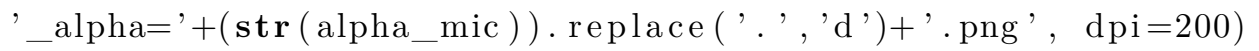

plt. show ()

\#\% Evolution equation for $R$ for multiple particles

if mesh_type='c' or Re_entrance $==0$ :

end $=$ timer ()

print ('time $\sqcup$ elapsed $\sqcup=$ ', end-start, 's' )

sys.exit ()

L11_proj $=\operatorname{project}(\mathrm{u}[0] . \mathrm{dx}(0)$, FS_DG0 $)$

L12_proj $=\operatorname{project}(\mathrm{u}[0] . \mathrm{dx}(1)$, FS_DG0 $)$

L21_proj $=\operatorname{project}(\mathrm{u}[1] . \mathrm{dx}(0)$, FS_DG0 $)$

L 22_proj $=\operatorname{project}(\mathrm{u}[1] . \mathrm{dx}(1)$, FS_DG0 $)$

$\mathrm{dt} \_$pathline $=1 \mathrm{e}-2$ 
$\mathrm{x}_{-} \mathrm{o}_{-}$lst $=[]$

theta_o_lst $=[]$

$\mathrm{x}$ _pathline_lst $=[]$

$y_{\text {_pathline_lst }}=[]$

aux_a_lst $=[]$

aux_b_lst $=[]$

aux_c_lst $=[]$

aux_d_lst $=[]$

r_dot_D_dot_r_lst $=[]$

$\mathrm{Rx}_{\text {_lst }}=[]$

$\mathrm{Ry}_{\text {_lst }}=[]$

L_pathline_lst $=[]$

$\mathrm{L} \_\mathrm{T}$ _pathline_lst $=[]$

U্ $\quad$ D_pathline_lst $=[]$

W_pathline_lst $=[]$

$\mathrm{rx}_{\text {_lst }}=[]$

$\mathrm{ry}_{\text {_ }} \mathrm{lst}=[]$

r_dot_r_lst $=[]$

abs_R_lst $=[]$

theta_lst $=[]$

theta_deg_lst $=[]$

time_lst $=[]$

$x_{\text {__pathline_star_lst }=[]}$

$y_{\text {_pathline_star_lst }=[]}$

Rx_star_lst $=[]$

Ry_star_lst $=[]$

abs_R_star_lst $=[]$

time_star_lst $=[]$

\#different y_o

x_o_lst .append $(-0.266)$

$\mathrm{x} \_$o_lst . append $(-0.266)$

x_o_lst .append $(-0.266)$

x_o_lst .append $(-0.266)$

x_o_lst .append $(-0.266)$

x_o_lst .append $(-0.266)$ 
if $l a m b==1$ :

y_o_lst . append $(0.06)$

y_o_lst . append (0.03)

y_o_lst .append $(0.02)$

y_o_lst .append (0.015)

y_o_lst . append $(0.01)$

y_o_lst . append $(0.0)$

elif $\operatorname{lamb}==0$ :

if Re_entrance $==3$ :

y_o_lst . append $(-0.01)$

y_o_lst . append $(-0.025)$

$\mathrm{y} \_$o_lst . append $(-0.035)$

y_o_lst . append $(-0.045)$

y_o_lst . append $(-0.05)$

y_o_lst . append $(-0.055)$

elif Re_entrance $==5$ :

y_o_lst . append $(-0.02)$

y_o_lst . append $(-0.025)$

y_o_lst . append $(-0.03)$

$\mathrm{y} \_$o_lst . append $(-0.035)$

y_o_lst . append $(-0.04)$

y_o_lst . append $(-0.045)$

elif Re_entrance $==10$ :

y_o_lst . append $(-0.005)$

y_o_lst . append $(-0.01)$

y_o_lst . append $(-0.015)$

y_o_lst . append $(-0.02)$

y_o_lst . append $(-0.025)$

y_o_lst . append $(-0.03)$

elif $\operatorname{lamb}==-1$ :

if Re_entrance $==3$ :

y_o_lst .append $(0.085)$

y_o_lst . append $(0.08)$

y_o_lst . append $(0.07)$

y_o_lst . append $(0.06)$

y_o_lst . append (0.05)

y_o_lst . append $(0.04)$

elif Re_entrance $==5$ :

y_o_lst . append $(0.07)$

y_o_lst . append $(0.06)$

y_o_lst . append $(0.05)$

y_o_lst . append $(0.02)$

y_o_lst . append $(-0.02)$

y_o_lst . append $(-0.04)$

elif Re_entrance $==10$ :

y_o_lst .append (0.045) 


$$
\begin{aligned}
& \text { y_o_lst . append (0.04) } \\
& \text { y_o_lst . append }(0.0375) \\
& \text { y_o_lst .append }(0.035) \\
& \text { y_o_lst . append }(0.033) \\
& \text { y_o_lst . append (0.03) }
\end{aligned}
$$

$\mathrm{kk}=$ len $\left(\mathrm{x} \_\right.$o_lst $)$

theta_o_lst.append ( $\mathrm{pi} / 4)$

theta_o_lst.append (pi $/ 4)$

theta_o_lst.append ( $\mathrm{pi} / 4)$

theta_o_lst.append (pi / 4$)$

theta_o_lst.append ( pi / 4)

theta_o_lst.append (pi $/ 4)$

for $\mathrm{k}$ in $\operatorname{range}(0, \mathrm{kk})$ :

$$
\mathrm{ds}=1 \mathrm{e}-5
$$

$x_{\text {_pathline }}=[]$

$y_{\text {_pathline }}=[]$

aux_a $=[]$

$\mathrm{aux \_ b}=[]$

aux_c $=[]$

aux_d $=[]$

r_dot_D_dot_r $=[]$

$\mathrm{Rx}=[]$

$\mathrm{Ry}=[]$

L_pathline $=[]$

L_T_pathline $=[]$

D_pathline $=[]$

W_pathline $=[]$

$\mathrm{rx}=[]$

$\mathrm{ry}=[]$

r_dot_r $=[]$

abs_R $=[]$

theta $=[]$

theta_deg $=[]$

x_pathline_star $=[]$ 


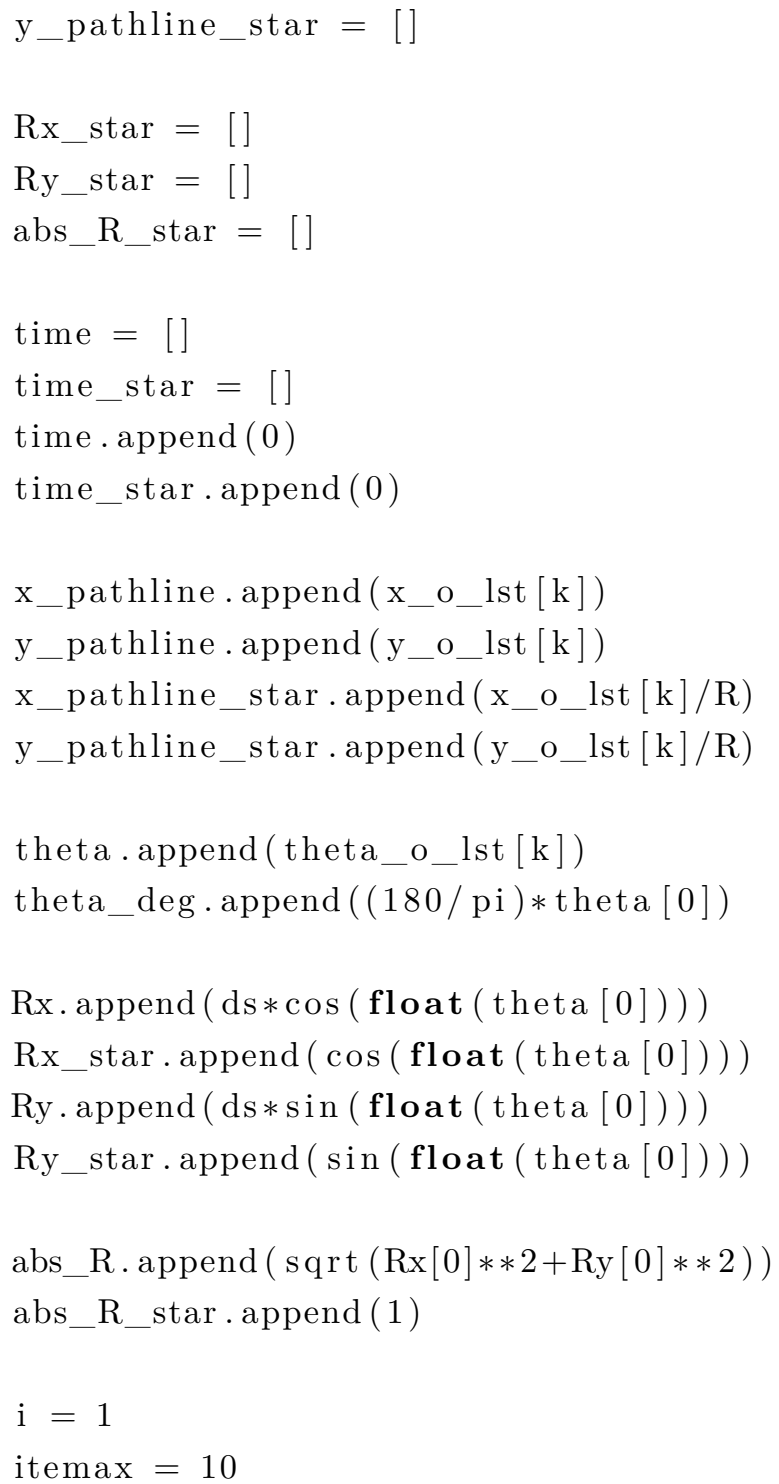

while (x_pathline $[i-1]<$ Len/2 and $x$ _pathline $[i-1]>-$ Len/2) and । (y_pathline $[i-1]<H / 2$ and $y$ _pathline $[i-1]>-H / 2)$ :

while $\left(\left(\left(x_{\text {_tpathline }}[\mathrm{i}-1]<\operatorname{Len} / 2\right.\right.\right.$ and $\mathrm{x}$ _pathline $[\mathrm{i}-1]>-$ Len $\left./ 2\right) \backslash$ and (y_pathline $[\mathrm{i}-1]<\mathrm{H} / 2$ and $\mathrm{y}$ _pathline $[\mathrm{i}-1]>-\mathrm{H} / 2)$ ) and $\backslash$ $((($ abs_R $[\mathrm{i}-1]>=10 * \mathrm{ds})==$ False $)$ and $)$ $(($ abs_R $[\mathrm{i}-1]<=0.01 * \mathrm{ds})==$ False $)))$ :

$\mathrm{x}$ _pathline .append $\left(\mathrm{x} \_\right.$pathline $[\mathrm{i}-1]+\backslash$ dt_pathline $*\left(\mathrm{u} 1\left(\mathrm{x} \_\right.\right.$pathline $[\mathrm{i}-1], \backslash$ y_pathline $[\mathrm{i}-1])[0]))$

y_pathline .append (y_pathline $[\mathrm{i}-1]+\backslash$ dt_pathline $*\left(\mathrm{u} 1\left(\mathrm{x} \_\right.\right.$pathline $[\mathrm{i}-1], \backslash$ y_pathline $[i-1])[1]))$

L_pathline .append (np. matrix ([ [L11_proj ( (x_pathline $[\mathrm{i}-1], \backslash$ $\left.\left.y_{\text {_pathline }}[\mathrm{i}-1]\right)\right)$, L12_proj ( $\left(x \_\right.$pathline $[\mathrm{i}-1], \backslash$ y_pathline $[\mathrm{i}-1]))], \quad$ ।

$\left[\mathrm{L} 21 \_\operatorname{proj}\left(\left(\mathrm{x} \_\right.\right.\right.$pathline $[\mathrm{i}-1], \mathrm{y} \_$pathline $\left.\left.[\mathrm{i}-1]\right)\right) \backslash$ 
L22_proj $\left(\left(x \_\right.\right.$pathline $\left.\left.\left.\left.\left.\left.[i-1], y \_p a t h l i n e[i-1]\right)\right)\right]\right]\right)\right)$

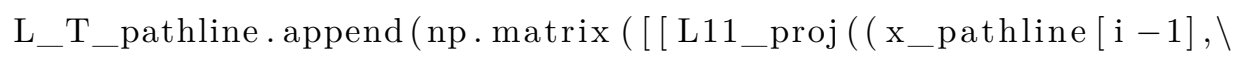

y_pathline $[\mathrm{i}-1]))$, ।

L21_proj ( $\left(x \_\right.$pathline $\left.[i-1],\right)$

y_pathline $[\mathrm{i}-1]))]$,

[L12_proj $\left(\left(x \_p a t h l i n e[i-1], y \_p a t h l i n e[i-1]\right)\right)$,

L22_proj $\left.\left.\left.\left.\left(\left(x \_p a t h l i n e[i-1], y \_p a t h l i n e[i-1]\right)\right)\right]\right]\right)\right)$

D_pathline .append $\left(0.5 *\left(\mathrm{~L}_{\text {__pathline }}[\mathrm{i}-1]+\mathrm{L}_{\text {_ }} \mathrm{T}\right.\right.$ _pathline $\left.\left.[\mathrm{i}-1]\right)\right)$

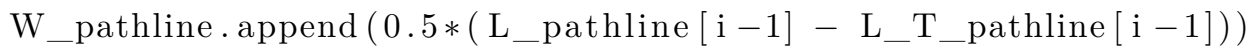

$\mathrm{rx} . \operatorname{append}(\operatorname{Rx}[\mathrm{i}-1] /(\operatorname{sqrt}(\operatorname{Rx}[\mathrm{i}-1] * * 2+\operatorname{Ry}[\mathrm{i}-1] * * 2)))$

ry $\cdot \operatorname{append}(\operatorname{Ry}[\mathrm{i}-1] /(\operatorname{sqrt}(\operatorname{Rx}[\mathrm{i}-1] * * 2+\operatorname{Ry}[\mathrm{i}-1] * * 2)))$

r_dot_D_dot_r.append $\left(\operatorname{rx}[\mathrm{i}-1] *\left(\operatorname{rx}[\mathrm{i}-1] * \mathrm{D} \_\right.\right.$pathline $[\mathrm{i}-1][0,0]+\backslash$

ry $\left.[\mathrm{i}-1] * \mathrm{D}_{\text {_p pathline }}[\mathrm{i}-1][1,0]\right)+\backslash$

ry $[\mathrm{i}-1] *\left(\operatorname{rx}[\mathrm{i}-1] * \mathrm{D} \_\right.$pathline $[\mathrm{i}-1][0,1]+\backslash$

ry $[\mathrm{i}-1] * \mathrm{D} \_$pathline $\left.\left.[\mathrm{i}-1][1,1]\right)\right)$

aux_a.append $\left(\mathrm{W} \_\right.$pathline $[\mathrm{i}-1][0,0]+\mathrm{G} \_$mic $*\left(\mathrm{D} \_\right.$pathline $[\mathrm{i}-1][0,0]-\backslash$

$\left(\mathrm{F} \_\right.$mic $/\left(\mathrm{F} \_\right.$mic +1$\left.)\right) *\left(\mathrm{r} \_\right.$dot_D_dot_r $\left.\left.[\mathrm{i}-1]\right)\right)-1$

$\left(\right.$ alpha_mic $/\left(\mathrm{F} \_\right.$mic +1$\left.\left.)\right)\right)$

aux_b.append $\left(\mathrm{W} \_\right.$pathline $[\mathrm{i}-1][0,1]+\mathrm{G} \_$mic $* \mathrm{D} \_$pathline $\left.[\mathrm{i}-1][0,1]\right)$

aux_c.append $\left(\mathrm{W} \_\right.$pathline $[\mathrm{i}-1][1,0]+\mathrm{G} \_$mic $* \mathrm{D} \_$pathline $\left.[\mathrm{i}-1][1,0]\right)$

aux_d.append (W_pathline $[\mathrm{i}-1][1,1]+\mathrm{G} \_$mic $*\left(\mathrm{D} \_\right.$pathline $[\mathrm{i}-1][1,1] \_$।

$\left(\mathrm{F} \_\right.$mic $/\left(\mathrm{F} \_\right.$mic +1$\left.)\right) *\left(\mathrm{r} \_\right.$dot_D_dot_r $\left.\left.[\mathrm{i}-1]\right)\right)-$ ।

$\left(\right.$ alpha_mic/ $\left.\left./\left(F_{\text {__mic }}+1\right)\right)\right)$

$\operatorname{Rx} . \operatorname{append}\left(\left(\operatorname{Rx}[\mathrm{i}-1] *\left(\left(1+0.5 *\right.\right.\right.\right.$ aux_a $[\mathrm{i}-1] * \mathrm{dt} \_$pathline $) /$

$(1-0.5 *$ aux_a $[\mathrm{i}-1] * \mathrm{dt}$ _pathline $)+\backslash$

$\left(0.25 *\right.$ aux_b $[\mathrm{i}-1] * \mathrm{aux} \_\mathrm{c}[\mathrm{i}-1] * \mathrm{dt} \_$pathline $\left.* * 2\right) / ।$

$\left(\left(1-0.5 *\right.\right.$ aux_a $[\mathrm{i}-1] * \mathrm{dt} \_$pathline $) * \backslash$

$\left(1-0.5 *\right.$ aux_d $[\mathrm{i}-1] * \mathrm{dt} \_$pathline $\left.\left.)\right)\right)+\backslash$

$\operatorname{Ry}[\mathrm{i}-1] *\left(\left(0.5 * \mathrm{aux} \_\mathrm{b}[\mathrm{i}-1] * \mathrm{dt}\right.\right.$ _pathline $) / ।$

$(1-0.5 *$ aux_a $[\mathrm{i}-1] * \mathrm{dt}$ _pathline $)+\downarrow$

$\left(0.5 *\right.$ aux_b $[\mathrm{i}-1] * \mathrm{dt} \_$pathline $*\left(1+0.5 *\right.$ aux_d $[\mathrm{i}-1] * \mathrm{dt} \_$pathline $\left.)\right) / \backslash$

$\left(\left(1-0.5 * a u x \_a[\mathrm{i}-1] * \mathrm{dt} \_\right.\right.$pathline $) *\left(1-0.5 *\right.$ aux_d $[\mathrm{i}-1] * \mathrm{dt} \_$pathline $\left.\left.)\right) \quad\right) * \backslash$

$\left(1-\left(0.25 *\right.\right.$ aux_b $[\mathrm{i}-1] *$ aux_c $[\mathrm{i}-1] * \mathrm{dt} \_$pathline $\left.* * 2\right) / \backslash$

$\left(\left(1-0.5 *\right.\right.$ aux_a $[\mathrm{i}-1] * \mathrm{dt} \_$pathline $) *\left(1-0.5 *\right.$ aux_d $[\mathrm{i}-1] * \mathrm{dt} \_$pathline $\left.\left.\left.)\right)\right) * *(-1)\right)$

Ry.append $\left(\left(1 /\left(1-0.5 *\right.\right.\right.$ aux_d $[\mathrm{i}-1] * \mathrm{dt} \_$pathline $\left.)\right) * \backslash$

$\left(\operatorname{Ry}[\mathrm{i}-1] *\left(1+0.5 * \operatorname{aux} \_\mathrm{d}[\mathrm{i}-1] * \mathrm{dt} \_\right.\right.$pathline $)+\backslash$

$\operatorname{Rx}[\mathrm{i}-1] *\left(0.5 * \operatorname{aux} \_\mathrm{c}[\mathrm{i}-1] * \mathrm{dt}\right.$ _pathline $)+\backslash$

$\operatorname{Rx}[\mathrm{i}] *\left(0.5 * \operatorname{aux} \_\mathrm{c}[\mathrm{i}-1] * \mathrm{dt} \_\right.$pathline $\left.)\right)$

abs_R .append ( $\operatorname{sqrt}(\operatorname{Rx}[\mathrm{i}-1] * * 2+\operatorname{Ry}[\mathrm{i}-1] * * 2))$

if $(\operatorname{Ry}[\mathrm{i}-1]>0$ and $\operatorname{Rx}[\mathrm{i}-1]>0)$ :

theta. $\operatorname{append}(n p \cdot \arctan (\operatorname{Ry}[\mathrm{i}-1] / \operatorname{Rx}[\mathrm{i}-1]))$

elif $(\operatorname{Ry}[\mathrm{i}-1]>0$ and $\operatorname{Rx}[\mathrm{i}-1]<0)$ :

theta. append (pi $-n p \cdot \arctan (\operatorname{Ry}[i-1] / \operatorname{Rx}[i-1]))$

elif $(\operatorname{Ry}[\mathrm{i}-1]<0$ and $\operatorname{Rx}[\mathrm{i}-1]<0)$ :

theta.append $(p i+n p \cdot \arctan (\operatorname{Ry}[i-1] / \operatorname{Rx}[i-1]))$

else :

theta. append $(3 *$ pi $/ 2+n p \cdot \arctan (\operatorname{Rx}[i-1] / \operatorname{Ry}[i-1]))$ 


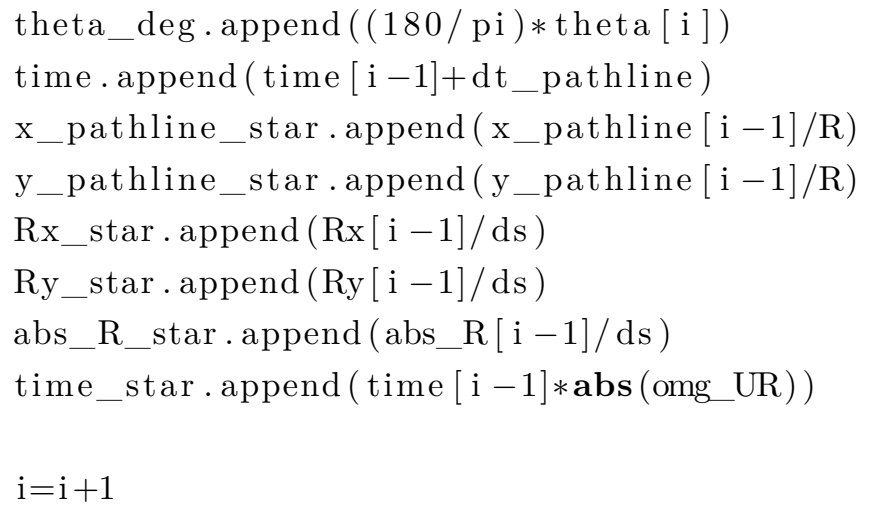

x_pathline $\cdot \operatorname{pop}()$

y_pathline.pop ()

D_pathline.pop ()

W_pathline. pop ()

Rx. pop ()

Ry. pop ()

abs_R.pop ()

theta.pop ()

theta_deg.pop ()

time.pop ()

x_pathline_star.pop ()

y_pathline_star.pop ()

Rx_star.pop ()

Ry_star.pop ()

abs_R_star.pop ()

time_star.pop()

x_pathline_lst.append ( $x \_$pathline $)$

y_pathline_lst.append (y_pathline)

D_pathline_lst.append (D_pathline)

W_pathline_lst.append(W_pathline)

Rx_lst.append ( $\mathrm{Rx})$

Ry_lst.append (Ry)

abs_R_lst.append (abs_R)

theta_lst.append (theta)

theta_deg_lst.append(theta_deg)

time_lst.append(time)

x_pathline_star_lst.append(x_pathline_star)

y_pathline_star_lst.append(y_pathline_star)

Rx_star_lst.append ( Rx_star)

Ry_star_lst.append (Ry_star)

abs_R_star_lst.append(abs_R_star)

time_star_lst.append(time_star) 


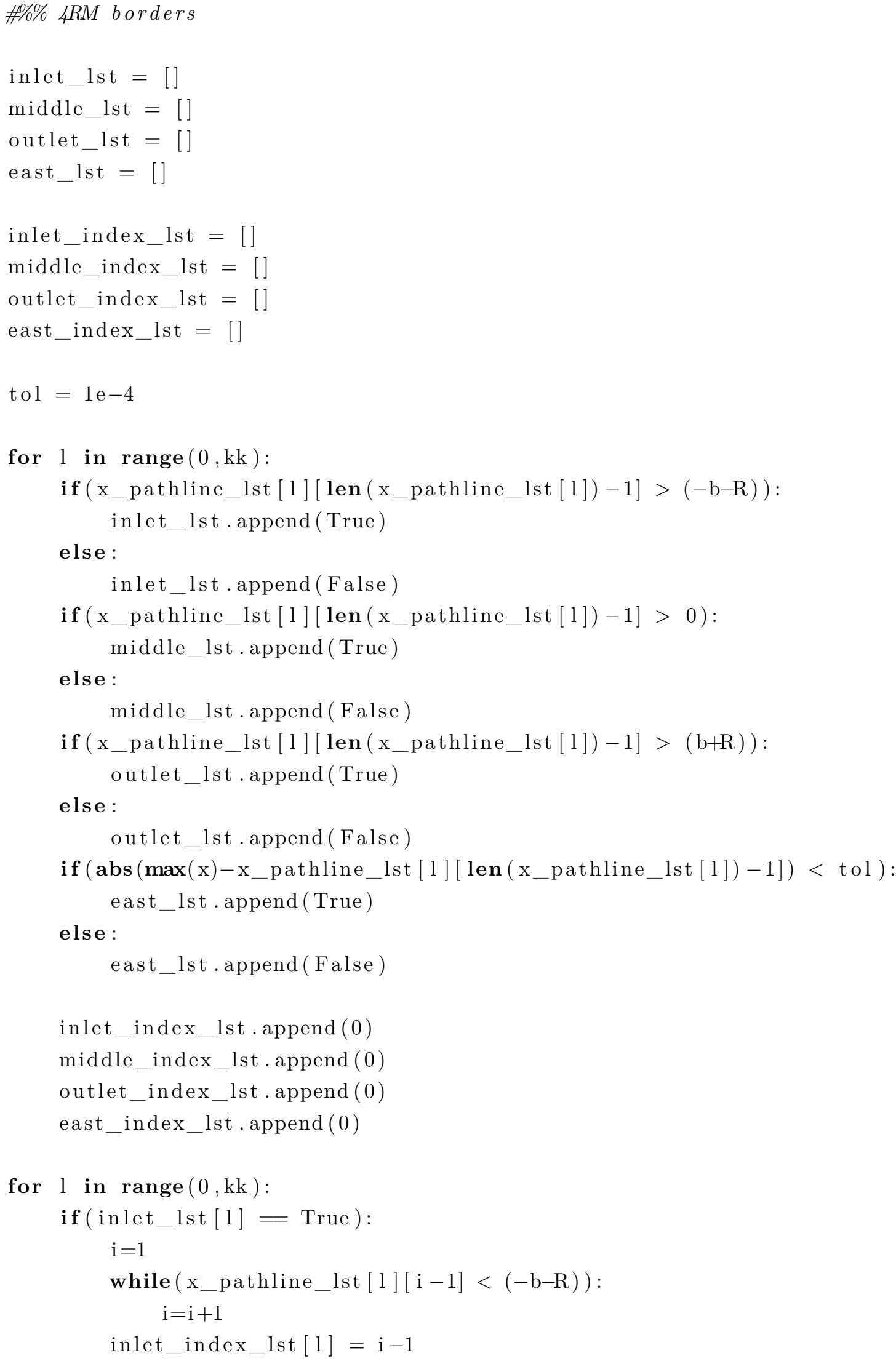




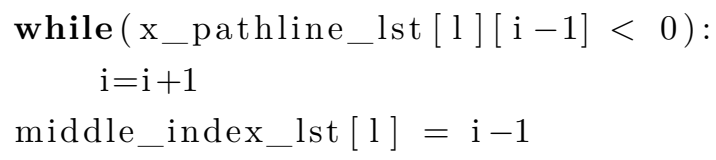

for $\mathrm{l}$ in range $(0, \mathrm{kk})$ :

if (outlet_lst [l]= True):

$\mathrm{i}=1$

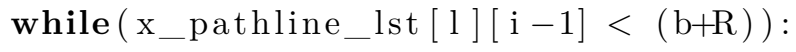

$$
\mathrm{i}=\mathrm{i}+1
$$

outlet_index_lst [l] $=\mathrm{i}-1$

for $\mathrm{l}$ in range $(0, \mathrm{kk})$ :

if ( east_lst [ l] = True ):

$\mathrm{i}=1$

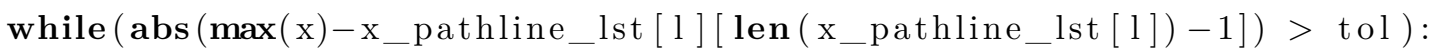
$\mathrm{i}=\mathrm{i}+1$

east_index_lst [l] $=\mathrm{i}-1$

\#\%\% Marking abs_R_star

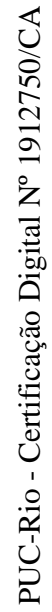

tol_abs_R_star $=1 \mathrm{e}-5$

\#Elongation

elongation_parameters $=$ []

elongation_indices $=[]$

e_aux $=[]$

elongation_parameters.append (3)

elongation_parameters . append (5)

elongation_parameters.append (7)

elongation_parameters.append (10)

for cont_e in range $(0, \mathrm{kk})$ :

elongation_indices.append ([])

e_aux.append $(0)$

cont_e $=$ cont_e +1

\#Compression

compression_parameters = []

compression_indices $=[]$

c_aux $=[]$

compression_parameters . append (1/9)

compression_parameters . append (1/25)

compression_parameters . append (1/49)

compression_parameters.append (1/100) 


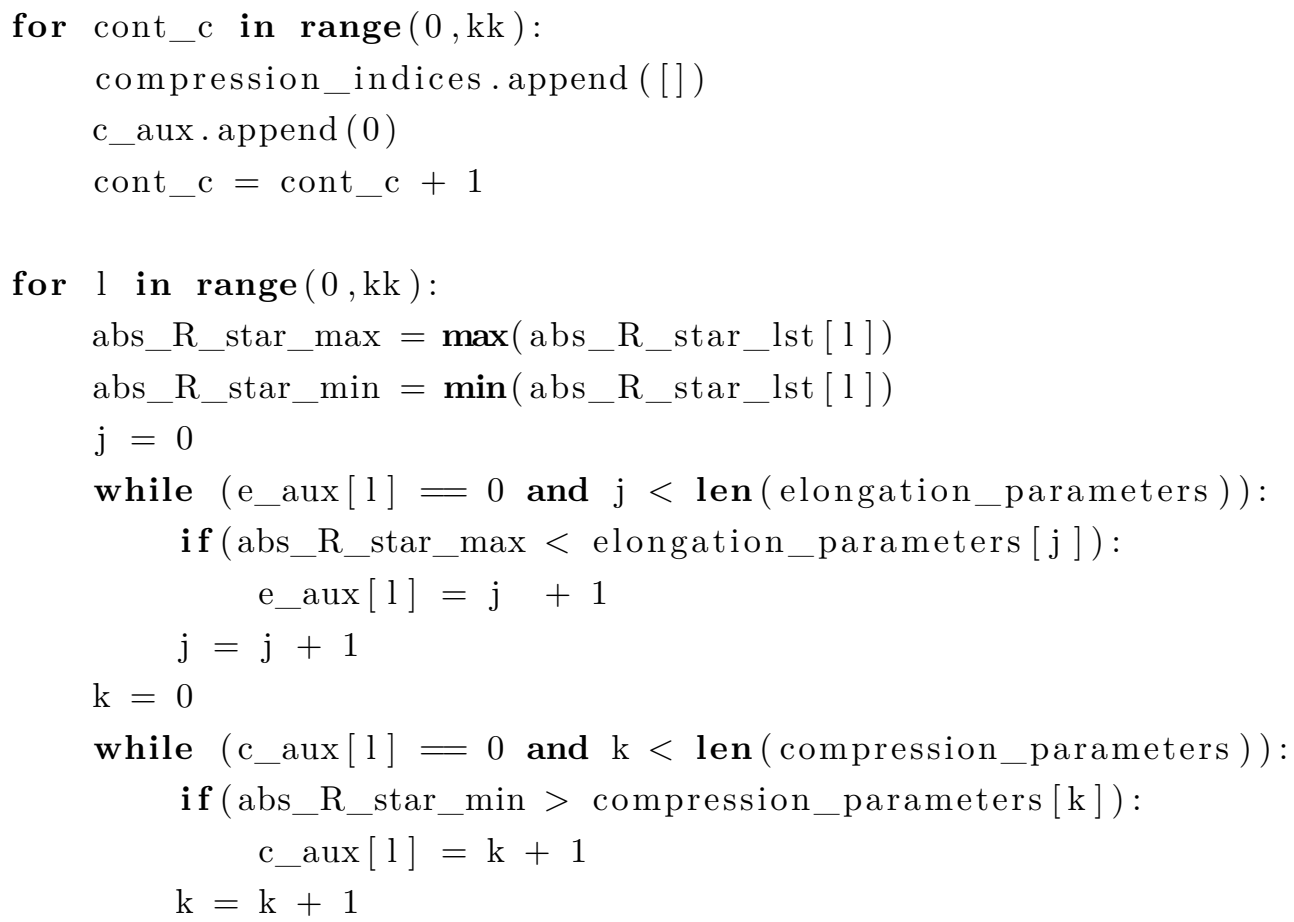

for $\mathrm{l}$ in range $(0, \mathrm{kk})$ :

if e_aux [l] = 1 :

pass

elif e_aux $[1]=2$ :

(elongation_indices [l]). append (next(e [0] ।

for e in enumerate(abs_R_star_lst [ l])

if e [1] > elongation_parameters [0]))

elif e_aux $[1]=3$ :

(elongation_indices [l]). append (next (e [0] ।

for e in enumerate(abs_R_star_lst[l]) if ।

e [1] > elongation_parameters [0]))

(elongation_indices [l]). append (next(e [0] ।

for e in enumerate(abs_R_star_lst[l]) if ।

e [1] > elongation_parameters [1]))

elif e_aux $[\mathrm{l}]=4$ :

(elongation_indices [l]). append (next(e [0] ।

for e in enumerate(abs_R_star_lst[l]) if ।

e [1] > elongation_parameters [0]))

(elongation_indices [l]).append(next(e[0])

for e in enumerate(abs_R_star_lst[l]) if ।

e[1] > elongation_parameters [1]))

(elongation_indices [l]).append(next(e[0]।

for e in enumerate(abs_R_star_lst[l]) if ।

e [1] > elongation_parameters [2]))

for $\mathrm{l}$ in range $(0, \mathrm{kk})$ :

if $c_{\_} \operatorname{aux}[1]=1$ : 


\section{pass}

elif c_aux [l] $=2$ :

(compression_indices [1]).append (next(c [0] ।

for $c$ in enumerate(abs_R_star_lst [1]) if ।

$c[1]<$ compression_parameters [0]))

elif c_aux $[1]=3$ :

(compression_indices [1]).append(next(c[0]।

for $c$ in enumerate(abs_R_star_lst[1]) if ।

$\mathrm{c}[1]<$ compression_parameters [0]))

(compression_indices [1]). append (next (c [0] ।

for $c$ in enumerate(abs_R_star_lst[l]) if ।

$c[1]<$ compression_parameters [1]))

elif c_aux $[\mathrm{l}]=4$ :

(compression_indices [1]).append(next(c [0]

for $c$ in enumerate(abs_R_star_lst[1]) if ।

c [1] < compression_parameters [0]))

(compression_indices [1]). append (next (c [0] ।

for c in enumerate(abs_R_star_lst[1]) if ।

$\mathrm{c}[1]<$ compression_parameters [1]))

(compression_indices [1]). append (next (c [0] ।

for $c$ in enumerate(abs_R_star_lst [l]) if ।

$\mathrm{c}[1]<$ compression_parameters [2]))

\#\% Plots Pathlines for different y_o

colors_lst $=[]$

colors_lst_2 $=[]$

colors_lst.append('bo')

colors_lst.append ('mo')

colors_lst.append ('go')

colors_lst.append ('co')

colors_lst.append ('ro')

colors_lst.append ('yo')

colors_lst_2.append ('b')

colors_lst_2 append ('m')

colors_lst_2 . append ('g')

colors_lst_2 . append ('c')

colors_lst_2.append ('r ')

colors_lst_2 . append ('y')

y_o_str_lst $=[]$

$\mathrm{y} \_$o_star_lst $=\left[\mathrm{x} / \mathrm{R}\right.$ for $\mathrm{x}$ in $\left.\mathrm{y} \_\mathrm{o} \_\mathrm{lst}\right]$ 
if $l a m b==1$ :

y_o_str_lst. $\operatorname{append}\left(\mathrm{r}^{\prime} \$\left\{\mathrm{y}_{\_} \mathrm{o}\right\}^{\widehat{*}} * \sqcup=\sqcup 3.15 \${ }^{\prime}\right)$

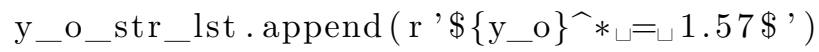

y_o_str_lst . append ( $\left.{ }^{\prime} \$\left\{y_{-} \_\right\}^{\wedge} * \sqcup=\sqcup 1.05 \$^{\prime}\right)$

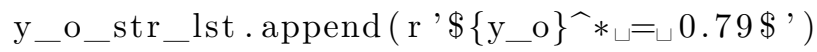

y_o_str_lst . append ( $\left.{ }^{\prime} \$\left\{y_{-} \_\right\}^{\wedge} * \sqcup=\sqcup 0.52 \$^{\prime}\right)$

y_o_str_lst . append ( $\left.\left.{ }^{\prime} \$\left\{\mathrm{y} \_\mathrm{o}\right\}\right\}^{*} * \sqcup=\sqcup 0 \$^{\prime}\right)$

elif $\operatorname{lamb}==0$ :

if Re_entrance $==3$ :

y_o_str_lst. $\operatorname{append}\left(\mathrm{r}^{\prime} \$\left\{\mathrm{y}_{\_} \mathrm{o}\right\}^{\widehat{*}} * \sqcup=\sqcup-0.52 \$^{\prime}\right)$

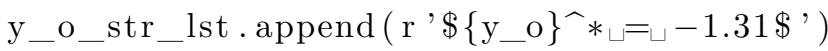

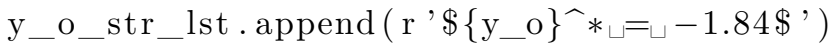

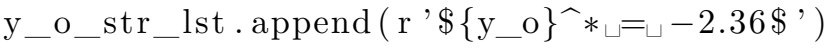

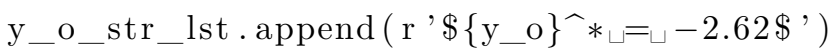

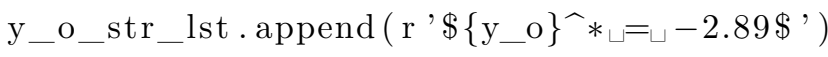

elif Re_entrance $==5$ :

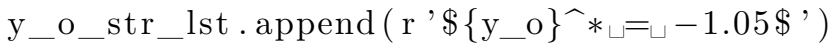

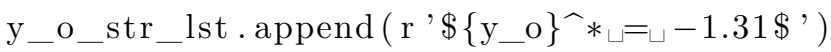

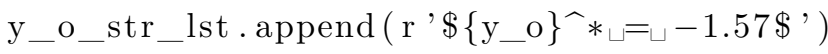

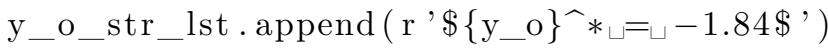

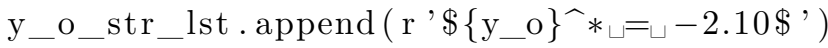

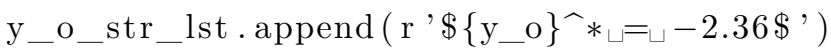

elif Re_entrance $==10$ :

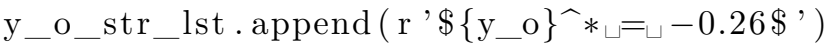

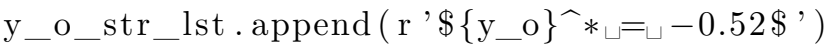

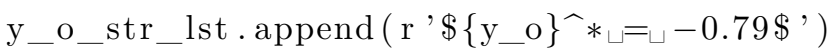

$\mathrm{y} \_$o_str_lst. $\operatorname{append}\left(\mathrm{r}^{\prime} \$\left\{\mathrm{y}_{\_} \mathrm{o}\right\}^{\widehat{*}} * \sqcup=\sqcup-1.05 \$^{\prime}\right)$

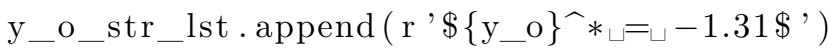

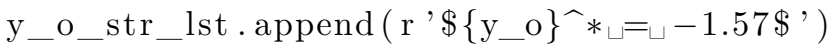

elif $\operatorname{lamb}==-1$ :

if Re_entrance $==3$ :

y_o_str_lst . append ( $\left.\left.{ }^{\prime} \$\left\{\mathrm{y} \_\mathrm{o}\right\}\right\}^{\wedge} * \sqcup=\sqcup 4.46 \$^{\prime}\right)$

$\mathrm{y} \_\mathrm{o} \_\mathrm{str} \_$lst. $\operatorname{append}\left(\mathrm{r}^{\prime} \$\left\{\mathrm{y} \_\mathrm{o}\right\}^{\wedge} *_{\llcorner}=\sqcup 4.20 \$^{\prime}\right)$

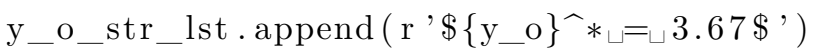

y_o_str_lst . append ( $\left.\mathrm{r}^{\prime} \$\left\{\mathrm{y} \_\mathrm{o}\right\}{ }^{\wedge} * \sqcup=\sqcup 3.15 \${ }^{\prime}\right)$

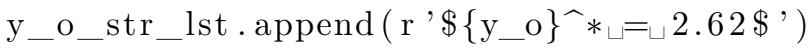

$\mathrm{y} \_\mathrm{o} \_\mathrm{str} \_$lst. $\operatorname{append}\left(\mathrm{r}^{\prime} \$\left\{\mathrm{y} \_\mathrm{o}\right\}^{\widehat{*}} * \sqcup=\sqcup 2.10 \$^{\prime}\right)$

elif Re_entrance $==5$ :

y_o_str_lst . append ( $\left.{ }^{\prime} \$\left\{y_{-} \_\right\}^{-} * \sqcup=\sqcup 3.67 \${ }^{\prime}\right)$

y_o_str_lst . append $\left(\mathrm{r}^{\prime} \$\left\{\mathrm{y} \_\mathrm{o}\right\}^{\wedge} * \sqcup=\sqcup 3.15 \$^{\prime}\right)$

$\mathrm{y} \_\mathrm{o} \_\mathrm{str} \_$lst. $\operatorname{append}\left(\mathrm{r} ' \$\left\{\mathrm{y} \_\mathrm{o}\right\}^{\wedge} *\left\llcorner=\sqcup 2.62 \${ }^{\prime}\right)\right.$

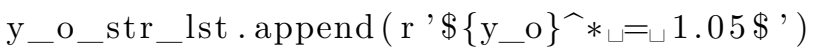

$\mathrm{y} \_\mathrm{o} \_$str_lst. $\operatorname{append}\left(\mathrm{r}^{\prime} \$\left\{\mathrm{y} \_\mathrm{o}\right\}^{*} *_{\sqcup}=-1.05 \${ }^{\prime}\right)$

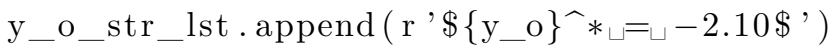

elif Re_entrance $==10$ :

y_o_str_lst . append $\left(\mathrm{r} ' \$\left\{\mathrm{y} \_\mathrm{o}\right\}^{\wedge} * \sqcup=\sqcup 2.36 \$^{\prime}\right)$

y_o_str_lst. $\operatorname{append}\left(\mathrm{r}^{\prime} \$\left\{\mathrm{y} \_\mathrm{o}\right\}^{\wedge} * \sqcup=\sqcup 2.10 \$^{\prime}\right)$ 


$$
\begin{aligned}
& \text { y_o_str_lst . append }\left(\mathrm{r}^{\prime} \$\left\{\mathrm{y} \_\mathrm{o}\right\}^{{ }^{*}} * \sqcup=\sqcup 1.98 \$^{\prime}\right)
\end{aligned}
$$

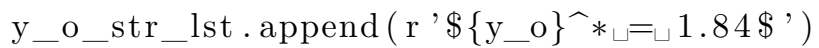

$$
\begin{aligned}
& \text { y_o_str_lst . append }\left(\mathrm { r } ' \$ \{ \mathrm { y } \_ \mathrm { o } \} ^ { \wedge } * \left\llcorner=\left\llcorner 1.73 \${ }^{\prime}\right)\right.\right. \\
& \text { y_o_str_lst.append }\left(\mathrm{r}^{\prime} \$\left\{\mathrm{y}_{\_} \mathrm{o}\right\}^{{ }^{*}} * \sqcup=\sqcup 1.57 \$^{\prime}\right)
\end{aligned}
$$

for $\mathrm{i}$ in range $\left(\operatorname{len}\left(\mathrm{y}_{\_} \mathrm{o}_{\text {_l }} \mathrm{lst}\right)\right)$ :

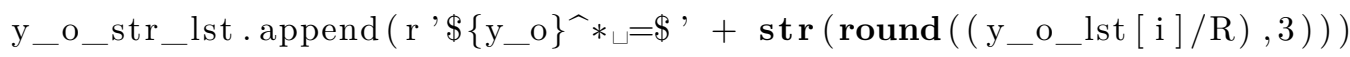

$\mathrm{i}=\mathrm{i}+1$

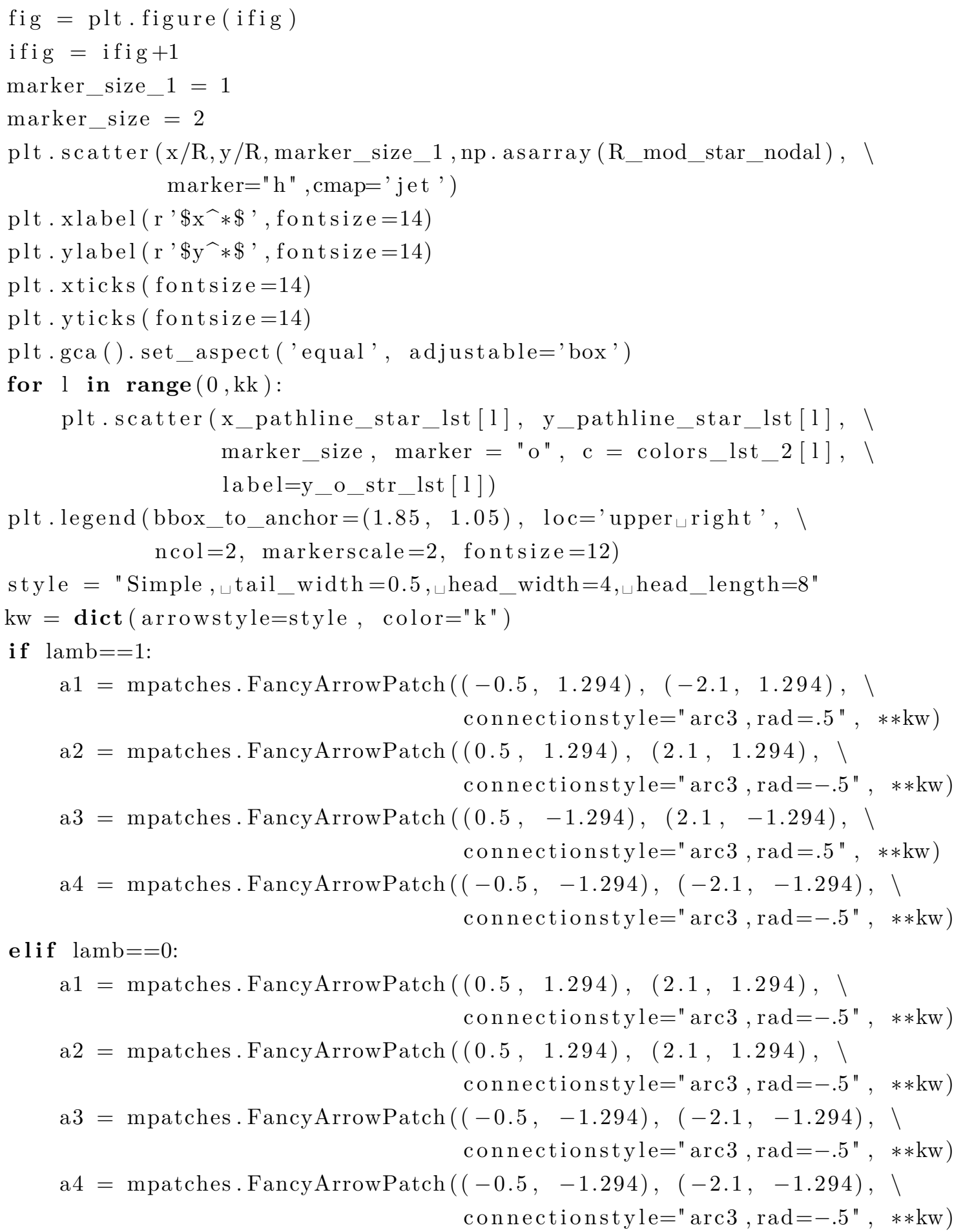




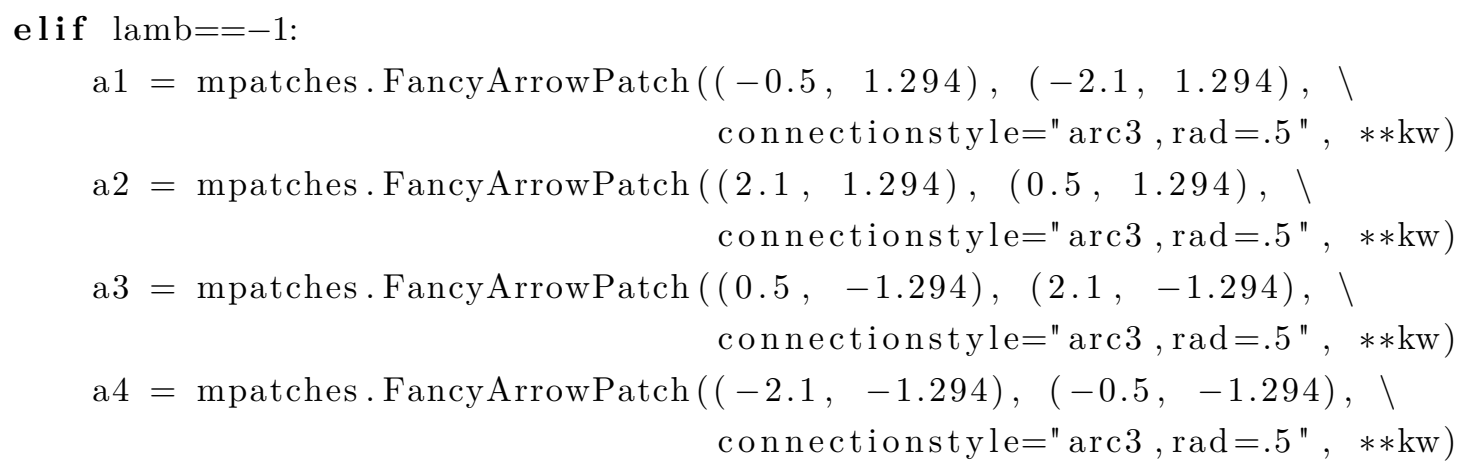

for a in $[\mathrm{a} 1, \mathrm{a} 2, \mathrm{a} 3, \mathrm{a} 4]$ :

plt.gca ().add_patch (a)

plt.savefig (results_path+'pathline_G=' $+\left(\operatorname{str}\left(G \_\right.\right.$mic $\left.)\right)$.replace (' .' , 'd') $+\backslash$

'_alpha='+(str (alpha_mic $))$.replace ('.', 'd') +1

'.png', dpi $=200$, bbox_inches='tight')

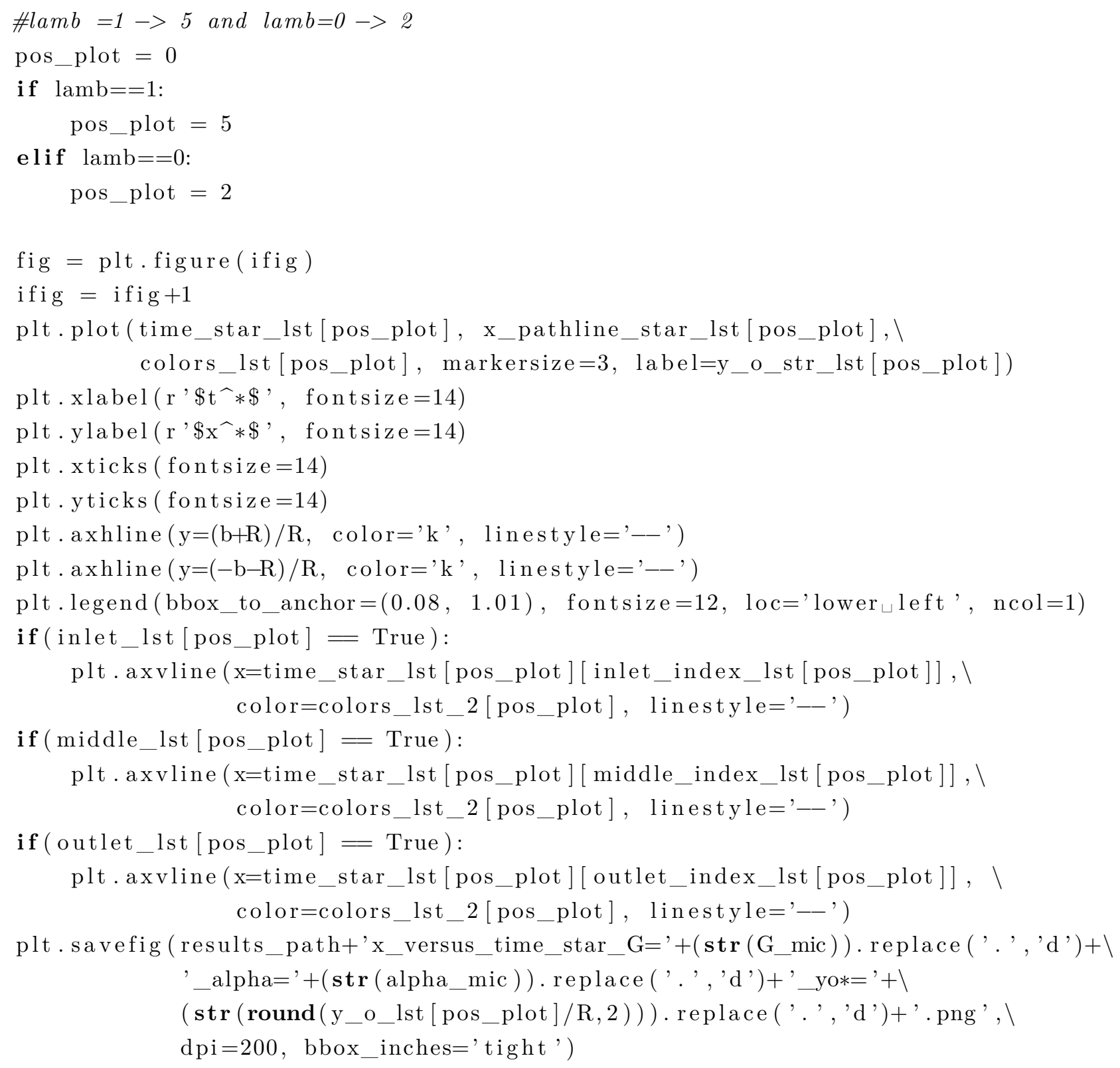




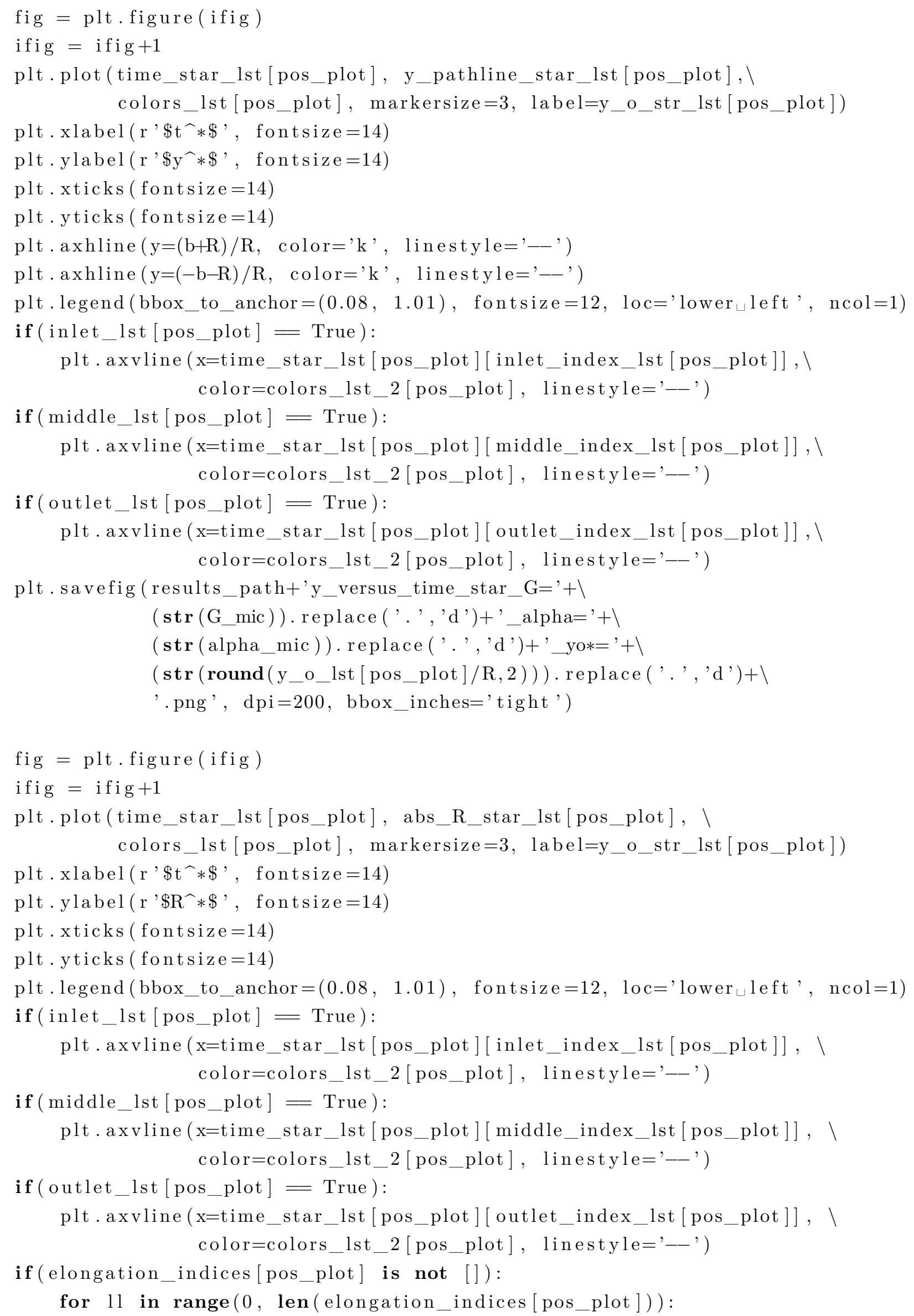


plt.plot(time_star_lst[pos_plot][elongation_indices [pos_plot][1l]], । abs_R_star_lst[pos_plot][ elongation_indices [pos_plot][1l]], । 'ko', markersize $=4$ )

if (compression_indices [pos_plot] is not []):

for 11 in range( 0, len(compression_indices[pos_plot])):

plt.plot(time_star_lst[pos_plot][compression_indices [pos_plot][1l]], $\backslash$

abs_R_star_lst[pos_plot][ compression_indices[pos_plot][1l]], 'ko')

plt.savefig (results_path+'abs_R_star_versus_time_star_G='+l

$\left(\operatorname{str}\left(\mathrm{G} \_\right.\right.$mic $\left.)\right)$.replace (' .', 'd ')+'_alpha $=$' +1

$(\operatorname{str}($ alpha_mic $))$.replace (' ' ', 'd ') +'_yo* $={ }^{\prime}+1$

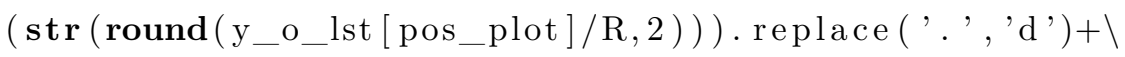

'.png', dpi $=200$, bbox_inches='tight')

\#\%\% Time elapsed

end $=$ timer ()

print ('time $\sqcup$ elapsed $\sqcup=$ ', end-start , 's ') 